\title{
Endocrine regulation of lipid mobilization in human obesity
}

Citation for published version (APA):

Verboven, K. (2017). Endocrine regulation of lipid mobilization in human obesity: unraveling the role of atrial natriuretic peptide . [Doctoral Thesis, Maastricht University]. Universiteit Hasselt en Maastricht University. https://doi.org/10.26481/dis.20170703kv

Document status and date:

Published: 01/01/2017

DOI:

10.26481/dis.20170703kv

Document Version:

Publisher's PDF, also known as Version of record

\section{Please check the document version of this publication:}

- A submitted manuscript is the version of the article upon submission and before peer-review. There can be important differences between the submitted version and the official published version of record.

People interested in the research are advised to contact the author for the final version of the publication, or visit the DOI to the publisher's website.

- The final author version and the galley proof are versions of the publication after peer review.

- The final published version features the final layout of the paper including the volume, issue and page numbers.

Link to publication

\footnotetext{
General rights rights.

- You may freely distribute the URL identifying the publication in the public portal. please follow below link for the End User Agreement:

www.umlib.nl/taverne-license

Take down policy

If you believe that this document breaches copyright please contact us at:

repository@maastrichtuniversity.nl

providing details and we will investigate your claim.
}

Copyright and moral rights for the publications made accessible in the public portal are retained by the authors and/or other copyright owners and it is a condition of accessing publications that users recognise and abide by the legal requirements associated with these

- Users may download and print one copy of any publication from the public portal for the purpose of private study or research.

- You may not further distribute the material or use it for any profit-making activity or commercial gain

If the publication is distributed under the terms of Article $25 \mathrm{fa}$ of the Dutch Copyright Act, indicated by the "Taverne" license above, 
2017 | Faculty of Medicine and Life Sciences

UM Maastricht University

\section{DOCTORAL DISSERTATION}

Endocrine regulation of lipid mobilization in human obesity:

unraveling the role of atrial

natriuretic peptide

\section{BIOMED}

BIOMEDISCH

ONDERZOEKSINSTITUUT

$\leftrightarrow$ UHASSELT

Promoters: $\quad$ Prof. Dr Dominique Hansen | UHasselt

$D / 2017 / 2451 / 21$

Prof. Dr Ellen Blaak | Universiteit Maastricht

Co-promoters: Prof. Dr Bert Op 't Eijnde | UHasselt

Dr Johan Jocken | Universiteit Maastricht 
The public defence will take place on Tuesday 4 July 2017 at 10.00 a.m.

Examination board:

Prof. Dr M.A. van Baak, voorzitter, UMaastricht, Nederland

Prof. Dr Dominique Hansen, promotor, UHasselt, België

Prof. Dr Ellen Blaak, promotor, UMaastricht, Nederland

Prof. Dr Bert Op ‘t Eijnde, copromotor, UHasselt, België

Dr Johan Jocken, UMaastricht, Nederland

Prof. Dr Virginie Bito, jurylid, UHasselt, België

Prof. Dr Paul Dendale, jurylid, UHasselt, België

Dr M.M.J. van Greevenbroek, jurylid, UMaastricht, Nederland

Prof. Dr P.C.N. Rensen, jurylid, Leids UMC, Nederland

Prof. Dr M. Rydén, jurylid, Karolinska University Institute, Stockholm, Sweden 


\section{Endocrine regulation of lipid mobilization in human obesity: unraveling the role of atrial natriuretic peptide}

\section{Dissertation}

to obtain the degree of Doctor at Maastricht University, on the authority of the Rector Magnificus Prof. dr. Rianne M. Letschert, and a degree of Doctor of Biomedical Science at the Transnational University Limburg, on the authority of the Rector Prof. dr. Luc De Schepper,

In accordance with the decision of the Board of Deans to be defended in public on Tuesday $4^{\text {th }}$ of July 2017 at 10.00 hours in Maastricht

\section{By Kenneth Verboven}




\section{Supervisors}

Prof. dr. E.E. Blaak, Maastricht University

Prof. dr. D.R.M.J Hansen, Hasselt University

\section{Co-supervisors}

dr. J.W.E. Jocken, Maastricht University

Prof. dr. B. Op 't Eijnde, Hasselt University

\section{Assessment Committee:}

Prof. dr. M.A van Baak, Maastricht University, Chair

Prof. dr. M. Rydén, Karolinska University Institute, Stockholm, Sweden

Prof. dr. P.C.N. Rensen, Leiden University Medical Center

Prof. dr. P. Dendale, MD, Hasselt University

Dr. V. Bito, Hasselt University

Dr. M.M.J. van Greevenbroek, Maastricht University 


\title{
Endocrine regulation of lipid
}

\section{mobilization in human obesity}

\author{
unraveling the role of atrial \\ natriuretic peptide
}


The research presented in this thesis was performed within NUTRIM School of Nutrition and Translational Research in Metabolism, which participates in the Graduate School VLAG (Food, Technology, Agrobiotechnology, Nutrition and Health Sciences), accredited by the Royal Netherlands Academy of Arts and Sciences, and within the Faculty of Medicine and Life Sciences of Hasselt University. Part of the research described in this thesis was funded by a research grant of the Research Foundation Flanders (FWO).

\section{NUTRIM}

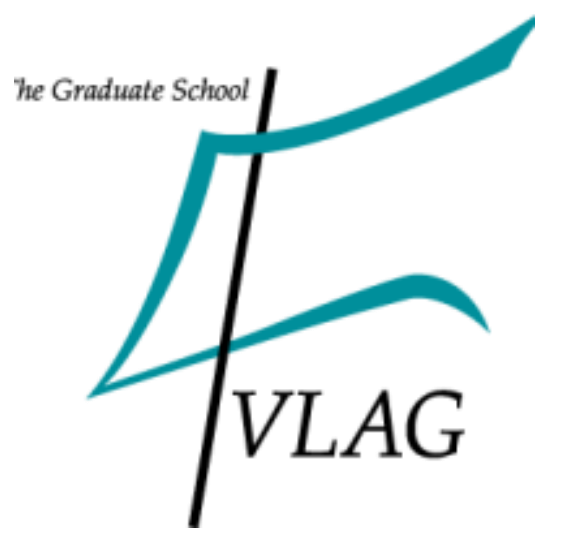

\section{Research Foundation \\ Flanders \\ Opening new horizons}

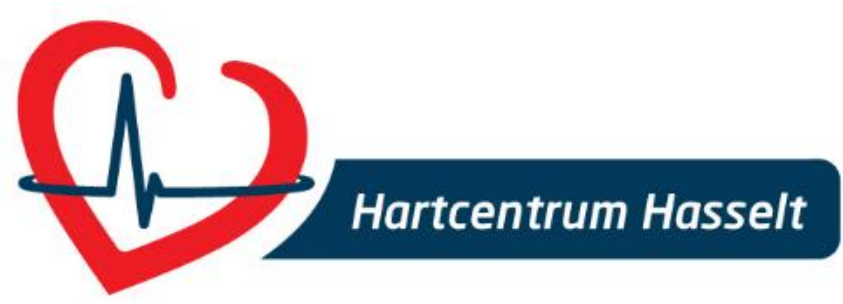

Financial support by Hartcentrum Hasselt for the publication of this thesis is gratefully acknowledged. 
Chapter 2 Natriuretic peptides in the control of lipid metabolism and insulin sensitivity

Chapter 3 Attenuated atrial natriuretic peptide-mediated lipolysis in subcutaneous adipocytes of obese type 2 diabetic men

Chapter 4 Abdominal subcutaneous and visceral adipocyte morphology, lipolysis and inflammation in relation to insulin resistance in obese humans

Chapter 5 Coordinated regulation of adipose tissue adrenergicnon-adrenergic-mediated lipolysis during exercise in lean and obese individuals: the effect of exercise training

Chapter 6 Adipose tissue lipolytic inhibition enhances the 175 glucoregulatory properties of exercise in type 2 diabetes patients

Chapter 7 General Discussion

Addendum Valorisation

Appendices Summary

Samenvatting

Curriculum Vitae

List of publications 

Chapter 1

GENERAL INTRODUCTION 


\section{Obesity}

In the last decades, the worldwide prevalence of overweight and obesity has risen enormously, affecting all ages and socioeconomic groups ${ }^{1-3}$. This unfortunate trend is driven by an increased consumption of energy-dense foods and the declining physical activity level, of which the relative contribution in obesity has been the subject for debate recently ${ }^{4-7}$. Traditionally, obesity is defined by the body mass index (BMI, defined as weight in kilograms divided by the square of height in meters $\left(\mathrm{kg} / \mathrm{m}^{2}\right)$ ), a simple surrogate measure used to determine excess body weight in adult populations and individuals. A BMI $\geq 25$ $\mathrm{kg} / \mathrm{m}^{2}$ indicates overweight and a BMI $\geq 30 \mathrm{~kg} / \mathrm{m}^{2}$ is classified as obesity ${ }^{1}$. However, recent findings suggest the inadequacy of BMI as a measure for general obesity categorization ${ }^{8,9}$ but there is still considerable controversy as to which measurement parameter most accurately defines unhealthy body fat distribution ${ }^{10}$. BMI based categorization revealed that worldwide $39 \%$ of the adults ( $>1.9$ billion) are overweight, being even $>60 \%$ in Europe, and $13 \%$ of the adults ( $>600$ million) are obese. These numbers are also observed in Belgium, where $67 \%$ of the adults deal with overweight and even $22 \%$ are diagnosed with obesity ${ }^{1}$. This trend is even more alarming as overweight and obesity is rising in children (even under the age of 5), reaching already more than 41 million children worldwide. It is clear that the increasing obesity prevalence is one of the major health concerns of our modern society since overweight and obesity increase the risk for developing metabolic complications such as insulin resistance (IR), type 2 diabetes mellitus (T2DM) ${ }^{11}$, hyperlipidemia, hypertension, atherosclerosis and cardiovascular diseases ${ }^{12}$. Moreover, obesity is associated with mental disorders ${ }^{13}$ and several types of cancer ${ }^{14,15}$, and stroke, congestive heart failure, pulmonary embolism, asthma, gallbladder disease and gout are more common in obese versus lean individuals 16. Increased body mass can also lead to biomechanical anomalies like obstructive sleep apnea ${ }^{17}$, chronic pain and osteoarthritis ${ }^{16}$, which together with the aforementioned comorbidities all result in a lower quality of life ${ }^{18}$ and an increased mortality ${ }^{19}$. Furthermore, obesity is likely to be associated with higher healthcare costs as it is related to more comorbidity, secondary care management and reduced ability for employment, indicating its high socio- 
economic burden. Hence, effective strategies are necessary to promote a healthier weight status, which has also economic benefits ${ }^{20}$.

\section{Inter-organ crosstalk in obesity-related insulin resistance}

The dysbalance between energy intake (that is, nutrient excess) and energy expenditure in obesity results in a disproportionate fat accumulation in adipose tissue ${ }^{21}$ and lipid overflow to non-adipose tissues like skeletal muscle ${ }^{22}$ and the liver ${ }^{23}$, thereby causing IR and inflammation, two central factors contributing to the development of cardiometabolic disease ${ }^{24-27}$ (Figure 1). With respect to IR, the ectopic lipid deposition disrupts insulin-mediated glucose uptake and glycogen storage, likely through direct interaction of lipid-intermediates with insulin signaling, thereby reducing insulin-stimulated translocation of cell membrane glucose transporters, or through activation of inflammatory pathways 28-32. Consequently, obesity-related insulin resistant states that progress towards the prediabetic state can be classified as impaired fasting glucose (IFG, fasting glucose $>5.6 \mathrm{mmol} / \mathrm{L}$ ) or impaired glucose tolerance (IGT, $2 \mathrm{~h}$ oral glucose tolerance test (OGTT)-derived glucose 7.8 - $11.0 \mathrm{mmol} / \mathrm{L}$ ), two intermediate states of impaired glucose metabolism preceding T2DM and associated with increased cardiovascular and chronic kidney disease risk ${ }^{33}$. Depending on the class (impaired fasting glucose, impaired glucose tolerance or a combination of both), these conditions may differ in the site of IR (that is, peripheral and/or hepatic IR) ${ }^{34-36}$, representing different etiologies towards T2DM ${ }^{37}$.

Long-term regulation and control of body weight (body composition) relies upon a large regulatory network integrating different metabolic organs and pathways with the aim of controlling energy intake, energy expenditure and substrate utilization. This network comprises a close inter-organ crosstalk between the adipose tissue, skeletal muscle, pancreas and liver in order to adapt to varying conditions (Figure 1). Normally, in the postprandial state dietary fatty acids are (for a large part) taken up and stored by the adipose tissue (mainly as triglycerides). However, in obesity the chronic positive energy balance enlarges the adipocytes (which become hypertrophic to accommodate this extra energy) which may lead to an impaired lipid buffering of the adipose tissue and IR $26,38,39$. This is further characterised by a disturbed lipoprotein lipase (LPL)- 
mediated lipid uptake ${ }^{40,41}$, an impaired hormone-mediated lipid breakdown through receptor and post-receptor defects ${ }^{42,43}$ and a reduced adipose tissue blood flow (leading to less triglyceride delivery to the adipose tissue) ${ }^{44,45}$. Once adipose tissue storage capacity is exceeded, lipids spill over to non-adipose tissues and are deposited ectopically ${ }^{46}$ when lipid supply exceeds fat oxidative capacity. There are indications that in particular skeletal muscle is characterised by an inability to increase fat oxidation upon increased supply, also indicated as metabolic inflexibility ${ }^{26}$. Besides the adipose tissue expandability hypothesis, a new concept, 'the personal fat threshold', was recently introduced to indicate that the physiological mechanisms underlying an individual's susceptibility to develop adverse effects of excess fat, such as T2DM, at a particular weight may vary between individuals 47 . With respect to ectopic skeletal muscle lipid accumulation, the overload of lipids is stored into lipid droplets as triglycerides (TAG) or lipid intermediates such as diacylglycerol (DAG), fatty acyl-CoA and ceramides, which interfere with insulin signaling 22,48 and thus may promote IR in the long term ${ }^{49-51}$. In this regard, the location and type (saturation) of bioactive lipid fractions as well as their turnover rate at the level of the skeletal muscle might be of major importance as opposed to the total amount per se $48,52,53$.

In the obese state, adipocyte hypertrophy is further characterized by a proinflammatory state in which an increased or altered production and secretion of pro-inflammatory cytokines (e.g. tumor necrosis factor-a (TNF-a), interleukin 6 (IL-6), IL-1 $\beta$, chemokines (e.g. monocyte chemoattractant protein-1 (MCP-1)) and adipokines (e.g. leptin, adiponectin) is present ${ }^{54,55}$. This increased local inflammation further attracts immune cells, such as macrophages ${ }^{56}$, thereby substantially increasing immune cell proportions in the obese adipose tissue ${ }^{57}$. In turn, these immune cells can switch from an anti-inflammatory (M2) to a proinflammatory (M1) phenotype (at least in animal studies) 58,59 , a phenotypic distinction that may be more complex in the human situation ${ }^{60}$, involving several other pathways besides its classical activation ${ }^{61}$. Hence, the present pro-inflammatory cells and/or processes leading to increase local and even systemic low-grade inflammation may lead to impairments in insulin signaling and contribute to the development of IR in peripheral insulin-sensitive metabolic tissues (e.g. muscle and liver) ${ }^{55,62}$ thereby increasing cardiometabolic risk. 


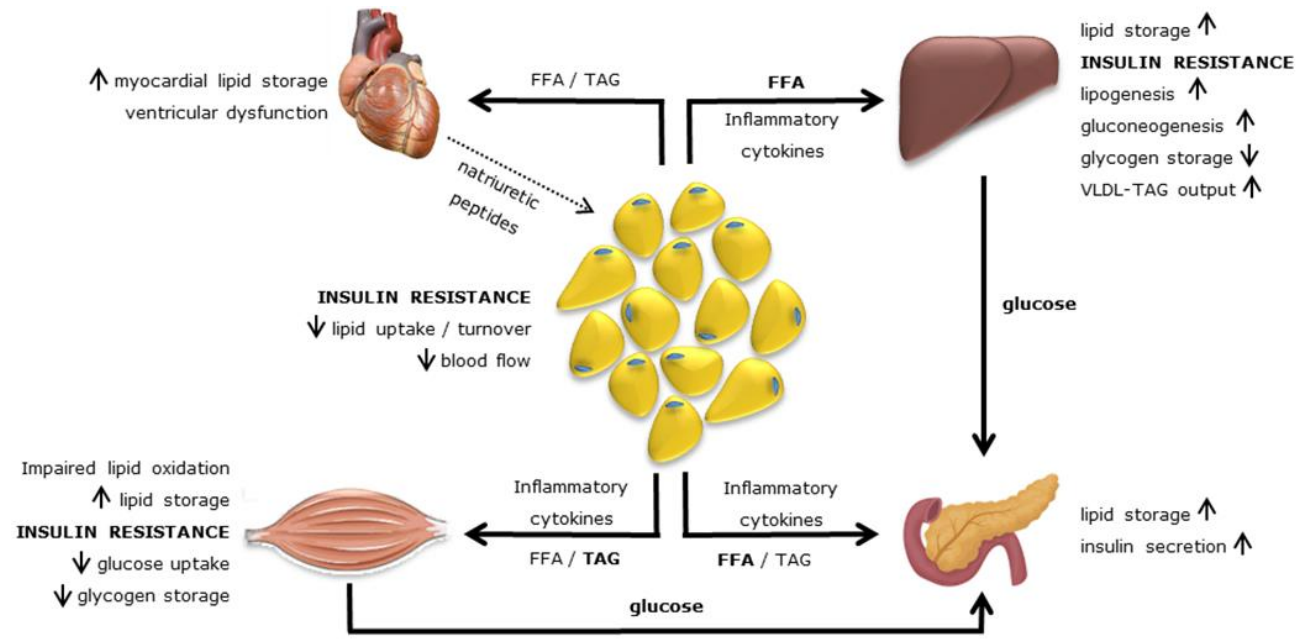

Figure 1 - Inter-organ crosstalk in the obese insulin resistant state. During long-term positive energy balance (when energy intake is much greater than energy expenditure), adipose tissue exceeds its lipid buffering capacity resulting in lipid overflow and lipid storage in non-adipose tissues (ectopic fat storage), which is associated with the systemic lowgrade inflammation and the development of insulin resistance. Substrate fluxes between tissues depicted by arrows (bold font indicates increased plasma concentrations and substrate fluxes in human obesity-related insulin resistance). FFA, free fatty acids; TAG, triacylglycerol; VLDL, very low density lipoprotein.

As already indicated before, altered storage, localization and composition of lipids and lipid-metabolites might result in lipotoxicity thereby interfering with insulin signaling and promoting IR $23,63,64$ in skeletal muscle ${ }^{31,65}$ and liver ${ }^{23,26}$. In addition to an increased supply of lipids to the skeletal muscle, disturbances in lipid handling may also contribute to the impaired insulin action at the level of the muscle. Skeletal muscle of obese insulin resistant individuals often displays a suboptimal fat oxidative capacity ${ }^{66}$, presumably related (at least in part) to an impaired fatty acid uptake, altered intramuscular lipolysis and attenuation of the mitochondrial capacity $42,49,67$. The resulting accumulation of lipid metabolites may lead to lipotoxicity and related IR in metabolically compromised conditions $31,37,66,68$. Furthermore, the capacity of pancreatic $\beta$-cells to produce insulin upon glucose stimulation is also blunted upon long-term lipotoxic conditions and ectopic pancreatic lipid accumulation ${ }^{69,70}$. Moreover, the liver is an important site for ectopic lipid accumulation, mainly in the postprandial phase as a consequence of the impaired insulin-mediated anti-lipolysis present in the obese 
state ${ }^{71}$. Indeed, several studies indicated the compelling association between hepatic lipid content and obesity ${ }^{72-74}$. In many obese individuals, ectopic lipid accumulation is also observed in and around the heart ${ }^{75}$, which is believed to be harmful for cardiac function ${ }^{76}$. The presence of ectopic lipids in multiple nonadipose tissues, besides their excessive storage within distinct adipose tissue depots, suggest that targeting adipose tissue lipid metabolism may be a possible effective method to prevent and/or treat metabolic disorders.

Recently, research indicated that the gut, in particular the gut microbiota composition and its products, may have an impact on host metabolism, thereby being an important player in inter-organ crosstalk. Studies have shown that alterations in the gut microbiota composition and function (microbiome) affect host adipose tissue, skeletal muscle and liver lipid and glucose metabolism. The gut microbiota has been proposed to be involved in the development of IR and impaired glucose metabolism 77 as it regulates host energy and substrate metabolism ${ }^{78-80}$, possibly via alterations in the production of bile acids, incretins, short chain fatty acids and inflammatory factors ${ }^{81-84}$. Nevertheless, a recent study showed that interference with adult microbiota by a 7-day antibiotic treatment has no clinically relevant impact on metabolic health in obese humans 85 , indicating that the microbiota may not play a physiologically significant role in all metabolic conditions. Of interest, gut-derived short chain fatty acids, which are formed after fermentation of dietary fibres, have anti-lipolytic potency, thereby possibly affecting peripheral lipid metabolism 86-88. Furthermore, angiopoietin-like protein 4 (ANGPTL4), a protein that is expressed and secreted by numerous cell types ${ }^{89}$ (including endocrine cells within the gut ${ }^{90}$, is able to modulate peripheral lipid metabolism under various conditions ${ }^{91-94}$.

As obesity increases the risk for both T2DM and cardiovascular disease, animal studies have postulated that alterations in either myocardial lipid uptake or fatty acid oxidative capacity may lead to the accumulation of various lipid intermediates that are linked to the progression of ventricular dysfunction, and thus cardiac lipotoxicity ${ }^{95}$. Indeed, a possible role of the heart with respect to inter-organ crosstalk becomes more plausible as the heart-adipose tissue connection has been of special interest in metabolic research during the last decade ${ }^{96}$. In particular, the heart is able to produce specific peptides (natriuretic 
peptides), which have beside cardiovascular effects ${ }^{97}$, several metabolic effects at the level of different insulin sensitive tissues ${ }^{98-100}$. The understanding of this inter-organ crosstalk (Figure 1) and the link between cardiovascular and metabolic effects is rapidly evolving as further examples of important physiological connections between tissues and systems are unveiled 96,99,100. However, with respect to the bi- or multi-directional communication between the heart and different metabolic tissues such as the adipose tissue, skeletal muscle, liver and pancreas, much work remains to be done, to identify future targets for the prevention and treatment of cardiometabolic diseases.

\section{Adipose tissue (dys)function in obesity-related insulin resistance}

The adipose tissue organ is composed of different discrete anatomical depots ${ }^{101}$, all with a different size and metabolic function 102,103. Regarding upper-body adipose tissue depots, the visceral adipose tissue (VAT) confers increased risk for metabolic complications and cardiovascular risk factors compared to subcutaneous adipose tissue (SCAT) ${ }^{104-107}$. Intriguingly, insulin resistant SCAT was suggested to contribute to ectopic VAT accumulation 108, presumably through a compensatory uptake of dietary lipids in VAT in case of an impaired SCAT buffering of dietary lipids ${ }^{109,110}$. This increased VAT may subsequently cause hepatic lipid accumulation and IR via increased delivery of lipids through the portal vein (portal hypothesis ${ }^{111}$ ) ${ }^{112}$. In contrast, impaired SCAT lipid buffering contributes for the largest part to systemic lipid overflow leading to ectopic lipid accumulation in peripheral tissues like skeletal muscle and IR.

During the last decades adipose tissue function, besides protecting internal organs and storing excessive energy in the form of fat, became the subject of numerous investigations in human (patho)physiology 30,113. Dysfunctional adipose tissue is a known concept, associated with an increased cardiometabolic risk and represented by an altered adipose tissue morphology, an impaired lipid buffering capacity (due to the presence of anomalies in lipid uptake, storage and release (lipid mobilization)) and modified secretion of endocrine, paracrine and autocrine signals that could contribute to local and systemic low-grade inflammation. All these characteristics of adipose tissue (dys)function will be discussed in detail below. 


\section{Adipocyte hypertrophy versus hyperplasia}

In humans, the total number of adipocytes is determined during childhood and adolescence. The adipocyte number remains stable during adulthood although cells are renewed throughout life ${ }^{114}$, implying proper neogenic and apoptotic capacity of the adipose tissue ${ }^{115}$. Data from early onset obesity revealed a yearly turnover of $\sim 8 \%$ of the adipocyte population ${ }^{116}$. However, these data might not reflect adipocyte turnover in adults who have gained weight over several years in adulthood, thereby first increasing their adipocyte size rather than the adipocyte number ${ }^{116}$. In line, during the average 10 year life span of human adipocytes, the triglyceride content is renewed 6 times, on average ${ }^{117}$. In case of long-term energy overload, adipocytes store this energy by expanding and increasing in size, a process called hypertrophy ${ }^{38}$. Hyperplasia is a second adaptive response of the adipose tissue ${ }^{118}$, an adipogenic process where pluripotent mesenchymal stem cells are committed to the preadipocyte lineage and/or preadipocytes undergo multiple mitosis cycles and differentiate into adipocytes ${ }^{119}$. When the hyperplastic potential of the adipose tissue is limited because of impaired differentiation, the expandability capacity decreases ${ }^{120-123}$. This in turn affects other tissues since adipocytes fully filled are extremely insulin resistant and therefore are restricted in their buffering capacity ${ }^{124,125}$. Of interest, in overweight and obese individuals a high amount of triglyceride is stored in the adipocytes which may result in a reduced triglyceride extraction, in particular in the postprandial state, as compared with lean individuals. This impaired lipid turnover reflects a reduced capacity to adjust to chronic lifestyle changes, thereby favoring fat mass accumulation ${ }^{117,126}$. In line, a low lipid turnover was associated with obesity ${ }^{126}$ and hypertrophic dysfunctional AT ${ }^{120}$. Indeed, this limited adipocyte and triglyceride turnover is an important characteristic of adipose tissue hypertrophy 120,126. Furthermore, this lipid removal rate was positively associated with the lipolytic capacity of adipocytes and was inversely related to IR ${ }^{117}$. Additionally, adipocyte hypertrophy has been shown to be a key phenotypic characteristic of non-obese T2DM patients ${ }^{127}$. Furthermore, many studies show adipocyte hypertrophy in obesity, IR and T2DM 116,128-130, which was associated with IR independently of adiposity indices 39,131,132. In obese T2DM patients, subcutaneous adipocyte number was lower compared to BMI-matched obese individuals without metabolic complications 
39,133-137. Yet, whether adipogenesis is an adaptive or maladaptive contributor to obesity-related IR or T2DM is still an open question ${ }^{120,138}$.

\section{Adipose tissue lipid mobilization}

In times of a negative energy balance, such as during fasting or physical exercise in order to fulfill other organs' needs, adipocytes are able to mobilize their stored (TAG) via adipocyte lipid mobilization (also called lipolysis). Hydrolysis of TAG into glycerol and free fatty acids (FFA) during lipolysis is achieved via the consecutive action of three lipases, which act sequentially: adipose triglyceride lipase (ATGL), hormone-sensitive lipase (HSL) and monoacylglycerol lipase (MGL) ${ }^{139-141}$. The main lipolytic regulators of human adipose tissue lipolysis are catecholamines (secreted by the adrenal medulla and sympathic nervous system) and natriuretic peptides (secreted by the heart), while anti-lipolysis is mainly mediated by insulin (Figure 2).

Adrenergic signaling in the adipose tissue is mainly mediated by the catecholamines adrenalin and noradrenalin (which are synthesized in the chromaffin cells of the adrenal gland medulla as a response to direct stimulation from the sympathetic nervous system (mediated by adrenocorticotropic hormone and/or cortisol) ${ }^{142}$ ) ${ }^{42,143-145}$. These hormones accomplish their lipolytic regulation by binding to different adrenergic receptors, for which their affinity (together with the relative abundance of each receptor on the adipocyte membrane) determines whether they stimulate or inhibit lipolysis. In this respect, $\beta$-adrenergic receptors on the adipocyte surface activate lipolysis, while anti-lipolytic signals are transferred by the $\alpha_{2}$-adrenergic receptors ${ }^{146}$. For $\beta$ adrenergic receptors, three subtypes exist $\left(\beta_{1}, \beta_{2}\right.$ and $\left.\beta_{3}\right)$ of which only the $\beta_{1}$ and $\beta_{2}$ are involved in human lipolysis regulation ${ }^{147-150}$. The $\beta_{3}$-adrenoceptor has been shown to be present in human ${ }^{151}$ as well as in rodent adipocytes ${ }^{152}$ and to participate in lipolysis ${ }^{151}$, via an ATGL-mediated mechanism (at least in mice) 153. A clear proof of a physiological relevance of the $\beta_{3}$-adrenoceptor in human obesity is still lacking ${ }^{154-156}$. However recent human data in young lean metabolically healthy individuals showed that the $\beta_{3}$-receptor agonist mirabegron can stimulate human brown adipose tissue thermogenesis and may be a promising treatment for metabolic disease ${ }^{157}$. These $\beta$-adrenergic 
receptors are coupled to a G-protein, containing a stimulating $\mathrm{G}_{\mathrm{S}}$ subunit which interacts with adenylyl cyclase upon stimulation ${ }^{158}$, thereby converting ATP to cyclic adenosine monophosphate (CAMP) resulting in elevated intracellular cAMP levels. This second messenger activates protein kinase A (PKA) ${ }^{159}$, which phosphorylates the lipid droplet-associated protein PLIN1 ${ }^{160}$ and cytosolic HSL 161-163 which becomes activated and translocated to the lipid droplet surface 164 where it docks on the phosphorylated PLIN1 and gains access to its substrate (that is, diacylglycerols) ${ }^{165,166}$. Phosphorylation of PLIN1 stimulates the release of comparative gene identification-58 (CGI-58), a co-activator of ATGL ${ }^{167,168 .}$ ATGL hereby becomes fully activated, resulting in the initiation of the lipolytic cascade and the generation of diacylglycerols serving as a substrate for HSL. In contrast, the $\alpha_{2}$-adrenergic receptors are coupled to a G-protein containing the inhibitory $\mathrm{G}_{\mathrm{i}}$ subunit, thereby inhibiting adenylyl cyclase and PKA activity ${ }^{158}$.

In the beginning of this millennium, natriuretic peptides (NPs), including atrial (ANP) and B-type (BNP) NPs, produced by the myocardium (by increasing NPs gene transcription and secretion of stored granules ${ }^{169}$ ) in response to myotube distension ${ }^{170}$ such as during exercise have been shown to be potent stimulators of human adipose tissue lipolysis ${ }^{171}$. By stimulating the guanylyl cyclase-linked type-A NP receptor (NPRA) on the adipocyte surface, NPs cause the conversion of intracellular GTP into cGMP. This results in the activation of protein kinase G (PKG), which activates the lipolytic cascade by the phosphorylation of PLIN1 and HSL ${ }^{172,173}$. Limited data is available with respect to ANP-mediated activation of ATGL ${ }^{174} \mathrm{NP}$-induced lipolysis is completely independent from the catecholamineinduced (CAMP/PKA mediated) lipolysis, as they rely on different pathways ${ }^{175,176}$ (Figure 2). However, an additive lipolytic effect occurs when human adipocytes are stimulated simultaneously with ANP and a beta-adrenergic agonist (e.g. isoproterenol) ${ }^{177}$. Unlike the CAMP-dependent pathway, NP-mediated lipolysis is unresponsive to the anti-lipolytic effects of insulin-mediated phosphodiesterase 3B (PDE3B) activation ${ }^{171,176}$. However, the NP pathway is counter-regulated via the hydrolysis of CGMP by other members of the PDE family, like PDE5 ${ }^{178,179 .}$ Indeed, PDE5 expression and activity have been found in isolated human adipocytes from both subcutaneous ${ }^{178}$ and visceral ${ }^{180}$ adipose tissue. However, this enzyme appears to be insufficient to control ANP-mediated lipolysis ${ }^{178 .}$ 
Hence, at present, details of the regulatory pathway counteracting ANPmediated lipolysis in vivo are incompletely understood ${ }^{179}$. In addition, binding of NPs to the type $C$ natriuretic peptide receptor (NPRC), also expressed on the adipocyte plasma membrane (amongst other tissues ${ }^{181-183}$ ) but lacking guanylyl cyclase activity, mainly facilitates scavenging of its ligands, internalization of ligand-receptor complexes and recycling of NPRC, together with lysosomal degradation of its ligands 182,184 . Expression of the functional NPRA and scavenging NPRC receptors appears to be regulated in an opposite way. In the fasted state, NPRA gene expression increases while NPRC gene expression decreases ${ }^{185}$. In contrast, high fat feeding and exposure to insulin greatly increased NPRC expression while reducing NPRA expression 183,186,187. The local tissue specific and systemic effects of NP are thought to depend on the ratio between the signaling receptor NPRA to the clearance receptor NPRC ${ }^{187,188}$. In addition to catecholamines and natriuretic peptides, a number of other hormones are known to participate in adipocyte lipolysis regulation, including growth hormone 189-193, cortisol 194-196, thyroid hormones 197, parathyroid hormone ${ }^{198,199}$, androgens ${ }^{200-204}$ and glucagon 205,206 . However, their impact on adipocyte lipolysis is much more reduced as opposed to the aforementioned major regulators and their pathways are briefly reviewed elsewhere ${ }^{207}$.

An alternative pathway that recently has been proposed to play a role in lipid breakdown is autophagy. Although autophagy (that is, a 'self-digestion' system that degrades unnecessary or dysfunctional cellular components to provide essential nutrients for cellular survival in times of energy deprivation) is generally seen as a non-selective process, studies clearly associate autophagy and the selective breakdown of lipids in murine adipocytes and hepatocytes, a process termed lipophagy 208-211. The potential hypotheses described in literature, indicating emerging roles of autophagy in cellular metabolism and metabolic diseases are beyond the scope of this thesis and are therefore referred to elsewhere ${ }^{212,213}$.

Insulin is considered being the main factor in the inhibition of adipocyte lipolysis. The binding of insulin to its receptor, which possesses intrinsic tyrosine kinase activity, leads to autophosphorylation and subsequent phosphorylation of the IRS1/2 ${ }^{214}$. Subsequently, this promotes phosphatidyl-inositol 3-kinase (PI3K) 
which increases phosphatidylinositol-3,4,5-triphosphate $\left(\mathrm{PIP}_{3}\right)^{215}$, thereby causing phosphorylation and activation of Akt (that is, PKB). Ultimately, Akt/PKB activates PDE3B and the degradation of CAMP ${ }^{216}$. Via this signaling pathway, insulin prevents the phosphorylation of HSL and PLIN1, thereby suppressing catecholamine-stimulated (not NP-stimulated) adipocyte lipolysis (Figure 2).

Besides the catecholamine-mediated $\alpha_{2}$-adrenergic receptor mediated activation of $G_{i}$ coupled proteins, several other factors arising from different cell types (such as stroma vascular fraction cells, sympathetic nerve terminals, the gut or adipocytes themselves) have been shown to inhibit lipolysis. In particular, GPR109A and GPR81, two G-protein coupled receptors with an inhibitory effect ( $G_{i}$ coupled receptors), are of special interest 217,218. GPR109A is highly expressed on adipocytes, binding ketone bodies of which plasma levels are increased during fasting ${ }^{218}$. GPR81 has a binding affinity for lactate and counterbalances catecholamine-induced lipolysis 217,219-222. Furthermore, short chain fatty acids receptors (GPR41 and GPR43 receptors) are present in human adipose tissue 223-225, via which their ligands have been shown to modulate adipocyte lipolysis by anti-lipolytic ${ }^{226}$ or lipolytic effects in vitro ${ }^{227}$. However, the impact of short chain fatty acids on lipolysis in human adipocytes needs to be investigated in more detail ${ }^{88}$. 


\section{Anti-lipolytic signals}

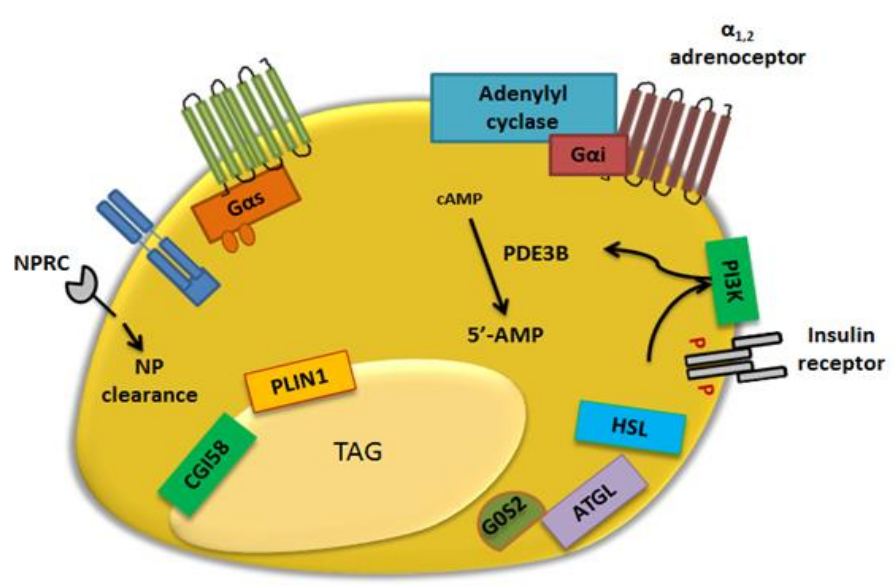

Lipolytic signals

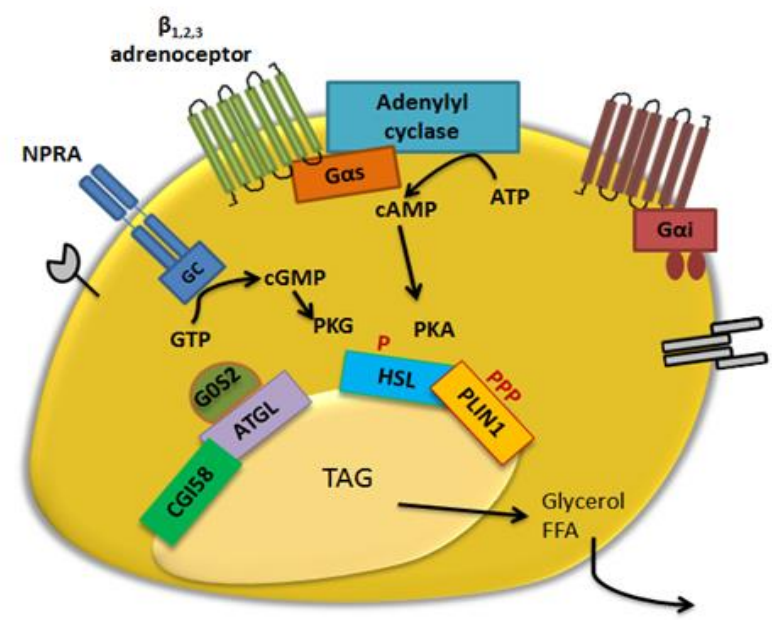

Figure 2 - Main regulators of adipose tissue lipolysis. Upper panel: anti-lipolytic signals. Insulin activation of PDE3B through a PI3K-dependent pathway and adenylyl cyclase inhibition through Gi protein-coupled receptor attenuate cAMP production and PKA activation. Binding of NPRC in anti-lipolytic conditions favors natriuretic peptide clearance. Lower panel: lipolytic signals. Catecholamines (adrenalin and noradrenalin) and natriuretic peptides (ANP, BNP) act through $\beta$ adrenoceptors and NPRA, respectively. Subsequently, PKA and PKG activation leads to HSL and PLIN1 phosphorylation. Phosphorylated HSL translocates to the lipid droplet surface to exert its function. Phosphorylated PLIN1 promotes lipid droplet fragmentation and ATGL co-factor CGI58 release, which interacts with and activates ATGL. ATGL, adipose triglyceride lipase; cAMP, cyclic adenosine monophosphate; cGMP, cyclic guanosine monophosphate; CGI58, comparative gene identification 58; FFA, free fatty acid; G0S2, $\mathrm{G}_{0} / \mathrm{G}_{1}$ switch gene $2 ; \mathrm{GC}$, guanylyl cyclase; HSL, hormone sensitive lipase; NPRA, natriuretic peptide receptor type $A$; NPRC, natriuretic peptide receptor type $C$; $P$, phosphate group; PDE3B, phosphodiesterase 3B; PI3K, phosphatidyl inositol 3 kinase; PKA: protein kinase A; PKG, protein kinase G; PLIN1, perilipin 1 ; TAG, triacylglycerol. 


\section{Depot-specific regulation of lipid mobilization}

Fat distribution, like android versus gynoid, rather than fat mass per se has profound implications with respect to metabolism and hence risk for cardiometabolic disease. With respect to adrenoceptors, intracellular lipases and lipid droplet-associated proteins, these are known to be differentially expressed in VAT and SCAT, contributing to depot-specific differences in lipolytic capacity 228-229. From in vitro studies, VAT adipocytes were shown to display lower basal (that is, non-stimulated) lipolysis but higher adrenergic responsiveness ${ }^{230-233 .}$ This aspect might be explained by a lower expression level of anti-lipolytic $\alpha_{2}$ adrenergic receptors, while $\beta$-adrenoceptors are expressed to a higher extent in the VAT, thus contributing to a higher adrenergic responsiveness of this depot 234,235 . With respect to anti-lipolysis, visceral adipocytes were reported to be more insulin resistant as compared to subcutaneous adipocytes ${ }^{236}$, implying that the relatively small contribution of VAT lipolysis to systemic FFA concentrations during the fasted state (as compared to SCAT) may be more pronounced during insulin-mediated suppression of lipolysis or in the postprandial state (reaching up to 40\%) 236-237. Physiologic factors addressing the cause(s) of these depot differences need to be explored further in vivo and might include changes in innervation, blood flow, or other factors affecting triglyceride synthesis and storage or inflammatory profile and adipokine secretion ${ }^{238}$. In addition, upper body subcutaneous depots (that are, femoral, gluteal and abdominal) have a higher responsiveness to $\beta$-adrenergic agonists, while lower body adipocytes are more responsive to $\alpha_{2}$-adrenergic agonists ${ }^{234-}$ 239. With respect to the anti-lipolytic effect of insulin in these different depots, data are inconclusive 240,241 .

Furthermore, the lipolytic effects of natriuretic peptides are thought to depend on the ratio between the signaling receptor NPRA to the clearance receptor NPRC 187,188. However, data about depot-specific natriuretic peptide receptors are currently lacking in lean individuals. In obese adipose tissue, VAT NPRA and NPRC mRNA expression was shown to be increased compared to SCAT In the obese state ${ }^{183}$. Moreover, NPRA expression is decreased in obese as compared to lean SCAT, both at mRNA and protein level 242,243 . 
The depot-specific differences in lipolytic activity may also be mediated by downstream differences in the lipolytic pathway, such as altered lipase or lipiddroplet-associated protein expression profiles. While ATGL mRNA expression is comparable between VAT and SCAT ${ }^{244-246}$, HSL mRNA expression and activity is shown to be higher in SCAT compared to VAT, which explains the higher lipolytic activity of the SCAT as compared to VAT ${ }^{247}$. However, other studies did not observe differences in HSL mRNA or protein expression between both depots ${ }^{248-}$ 251 , indicating that the direction of the adipose tissue depot difference in lipase expression is unclear. Of interest, higher lipase protein expression (that is, HSL and ATGL) has been shown for large adipocytes as opposed to small adipocytes from the same subject ${ }^{252}$, indicating the important association between adipocyte size and lipase expression/activity.

The lipid droplet-associated protein PLIN1, the major important perilipin family member involved in the regulation of adipose tissue lipolysis ${ }^{253}$, differs in depot expression as well. In the obese state, PLIN1 mRNA expression has been reported to be increased in VAT ${ }^{254,255}$, but unaffected in SCAT ${ }^{254}$. In contrast, PLIN1 protein expression is reduced in both obese VAT and SCAT as compared to lean adipose tissue depots ${ }^{254}$, and among obese individuals, the protein levels in SCAT are lower in insulin resistant compared to insulin sensitive individuals ${ }^{256}$.

\section{Lipolytic impairments in obesity-related insulin resistance}

It has been suggested that plasma FFA concentrations increase in proportion with the degree of obesity ${ }^{257}$. This increase was believed to result from an increased basal lipolysis in the obese state, since plasma FFA are predominantly derived from adipose tissue lipolysis ${ }^{140}$. However, the question whether an FFA overload exists in obesity became a matter of discussion ${ }^{258}$. In light of basal (that is, spontaneous or non-stimulated) lipolysis, data are inconsistent showing basal lipolysis to be increased ${ }^{259}$, unchanged ${ }^{260}$ or even decreased ${ }^{261,262}$ in obese SCAT, depending on the degree of IR or method of expression. It might be evident to state that in obese individuals basal lipolysis in fasting conditions (measured as plasma FFA concentrations) is increased due to the increased adipose tissue mass. However, plasma FFA concentrations have shown to be not increased in proportion to the amount of fat mass, indicating basal lipolysis to be 
even lower in obese versus lean individuals 258,262-264. Nevertheless, the importance of basal lipolytic rate in IR was corroborated by a bariatric surgeryinduced weight loss study, in which obese individuals with the highest decrease in basal lipolysis (as determined by spontaneous glycerol release from isolated mature adipocytes in vitro) were those with the greatest improvement in insulin sensitivity ${ }^{265}$. Adipose tissue IR (that is, the blunted suppression of lipolysis during hyperinsulinemia), has been associated with glucose intolerance and adiposity 43,266,267. Also, in human cohorts with a wide spectrum of adiposity, basal lipolysis (spontaneous glycerol release from isolated mature adipocytes in vitro) has been proven to be a determinant of variations in insulin sensitivity ${ }^{265}$. Accordingly, the SCAT of obese non-diabetic and type 2 diabetic individuals is resistant to the anti-lipolytic effect of insulin. Recently, a cohort with a wide ranging in glucose tolerance showed that fasting adipose tissue $I R$, quantified by means of the fasting FFA times fasting insulin 268-272, progressively rises over the span of glucose tolerance (ranging from normal glucose tolerance to impaired glucose tolerance to T2DM) ${ }^{273}$. Furthermore, the enlargement of adipocytes (that is, hypertrophy) was suggested to be a major contributor to IR ${ }^{126,265}$, a phenotype that has been linked to increased lipolytic rates as well, independent of BMI or age 252,274 .

In line with the above-mentioned findings, an in vivo blunted whole-body catecholamine-induced lipolysis has been observed in obese individuals 42,275-280. In vitro the site of this reduced catecholamine-stimulated lipolytic response was shown to be the abdominal SCAT ${ }^{147,259,281-285}$. Only some ${ }^{278}$, but not all ${ }^{286,287}$, in situ microdialysis studies support this observation. It is however unclear whether these defects in lipolysis are of primary origin or rather a secondary result in the etiology of obesity and related IR 42,140 . Of interest, lipolytic defects may be causally related to obesity development, a theory that has been observed in childhood onset obesity 288,289 and in first-degree relatives of obese individuals ${ }^{290}$. On the other hand, reductions in lipolytic activity have been proposed to be secondary to obesity development by others ${ }^{126}$, maybe caused by hyperinsulinemia ${ }^{266}$. Regarding ANP-mediated lipolysis, Rydén et al. has recently showed an impaired ANP-mediated lipolysis in vitro and in situ in human SCAT of obese women compared to non-obese counterparts ${ }^{242}$. Of interest, ANP-mediated lipolysis was comparable between subcutaneous 
adipocytes compared to visceral adipocytes of obese individuals ${ }^{291}$. However, these studies investigating depot-specific ANP-mediated lipolysis did not include lean individuals, nor obese individuals with type 2 diabetes. The decreased NPinduced lipolysis may be the result of an impaired receptor signaling in subcutaneous adipocytes ${ }^{242}$, an anomaly that might be caused by the hyperinsulinemic state that is frequently observed with obesity $174,183,187,243,292$. The lipolytic impairments summarized here may be an early factor in the development of increased fat stores and obesity-related metabolic complications. Therefore, a better insight into the role of these anomalies in obesity pathophysiology as well as lipolytic endocrine regulation is warranted.

\section{Adipose tissue inflammation in obesity}

The presence of immune cells in adipose tissue has been known for decades ${ }^{293}$. The first report providing the basis for the hypothesis that local inflammation was present in the obese adipose tissue dates from the early nineties, where Hotamisligil et al. ${ }^{294}$ reported TNF- $\alpha$ gene and protein expression to be increased in obese compared to lean adipose tissue, suggesting their role in whole-body insulin sensitivity. The origin of this pro-inflammatory cytokine is believed to be adipose tissue macrophages ${ }^{295,296}$ surrounding dead or apoptotic adipocytes 297 . Moreover, several adipose tissue-derived cytokines, such as monocyte chemoattractant protein 1 (MCP-1) and IL-6, are known to be altered in the obese state 298,299 . These cytokines initiate the migration of monocytes from the circulation into the adipose tissue, where they further differentiate into macrophages ${ }^{300}$, contribute to maintain adipose tissue inflammation and attract other immune cells ${ }^{301}$. Moreover, different immune cells are activated within hypertrophic adipose tissue (in case of resident immune cells) or attracted to it, especially in the VAT (mainly based on rodent studies) ${ }^{302,303 . ~ U p o n ~ m i g r a t i n g, ~}$ macrophages populate the adipose tissue, reaching cell proportions up to $50 \%$ in obese individuals ${ }^{295}$, in order to remove cell debris, remodel the adipose tissue and serve as a lipid buffer in order to reduce lipid overload ${ }^{304}$. Indeed, FFA serve as ligands for the toll-like receptor 4 (TLR4) complex ${ }^{305}$, activate classical inflammatory response, and drive adipose tissue macrophage accumulation 306,307. Switching macrophage phenotype is also associated with adipose tissue

inflammation ${ }^{308-310}$ because macrophages can exist along a spectrum of 
phenotypes 311 , the poles being M1 (or classically-activated) and M2 (or alternatively-activated) ${ }^{312}$. M1 macrophages display a pro-inflammatory appearance (characterized by expression of cytokines like TNF- $\alpha$, IL- 6 and IL-1 $\beta$ ) while M2 macrophages are involved in tissue remodeling and wound healing, expressing anti-inflammatory cytokines like IL-10 and IL-1 receptor antagonist. During obesity progression, more inflammatory M1 macrophages infiltrate the adipose tissue, causing IR and maintaining inflammation ${ }^{313}$, while adipose M2 macrophage numbers remain stable (or even slightly increase), resulting in a vicious inflammatory cycle ${ }^{314}$. Following these processes, a shift towards an increased, pro-inflammatory M1/M2 ratio culminates 299,315,316. The above mentioned division in inflammatory M1 macrophages and anti-inflammatory M2 macrophages is merely based on animal studies whilst in humans, the distinction between different macrophage phenotypes may be more complex, as other activation pathway(s) may define their properties ${ }^{60,113,311}$.

Still, the dynamic changes in which different immune cells initiate and maintain adipose tissue inflammation are incompletely understood. Other immune cells beside macrophages are likely to be involved in the initiation of local inflammation, all exerting negative effects on insulin sensitivity ${ }^{303}$. Indeed, $T$ lymphocytes ${ }^{317-319}$, B lymphocytes ${ }^{320,321}$, NK cells ${ }^{322}$, neutrophils ${ }^{323}$, dendritic cells ${ }^{324}$ and mast cells ${ }^{325}$ have been found to reside in the adipose tissue in the obese state, where they may contribute to adipose tissue inflammation and IR. Of interest, $T$ and $B$ lymphocytes gained considerable attention because of the observed early influx into the adipose tissue upon high-fat diet in mice ${ }^{317,326}$, thereby preceding macrophage infiltration into the adipose tissue ${ }^{309,326}$. The proinflammatory features of B cells in a high-fat diet setting was also reported in mice ${ }^{321}$, which can also contribute to a decreased glucose tolerance via orchestrating $\mathrm{T}$ cell populations and releasing pathogenic antibodies ${ }^{320}$. Furthermore, reduced proportions of regulatory $\mathrm{T}$ cells in adipose tissue, and thus a downscaled dampening of proinflammatory signaling, have been found to precede macrophage accumulation in abdominal (epididymal) adipose tissue in mice and omental adipose tissue in obese individuals ${ }^{327}$. This depletion of regulatory $T$ cells has been implicated in IR ${ }^{328,329}$, presumably by exerting their anti-inflammatory properties thereby preventing accumulation of pro- 
inflammatory cells in the adipose tissue, as was shown in murine visceral adipose tissue ${ }^{329}$. In addition, it was recently proposed that NK cells might be of importance in obesity-related IR and adipose tissue inflammation and it has been shown that manipulations of NK cells (either with neutralizing antibodies or genetic ablation) altered metabolic outcomes in obese animals ${ }^{322}$. These data indicate a strong interplay between adaptive (e.g. B cells and T cells) and innate immunity (e.g. macrophages and NK cells), which determines the inflammatory characteristics of obese adipose tissue. Therefore, gaining insights in inflammation-related concepts in the pathogenesis of obesity-induced IR is important, especially because the early local inflammation is likely to escalate when not counteracted, thereby contributing to systemic (low-grade) inflammation, IR and lipotoxicity in tissues like skeletal muscle and liver 30,113,330. Of interest, VAT inflammation (as demonstrated by increased macrophage infiltration in VAT) was proposed to be the strongest correlate of IR in obese human with a wide range of insulin sensitivity 331,332 . Notwithstanding the pathogenic properties of adipose tissue inflammation, one should keep in mind that these immune cells also interact with adipose tissue lipid metabolism, affecting the lipid storage or buffering capacity of the adipose tissue and lipolysis 304,333 , and promote adipogenesis ${ }^{334}$. The latter data indicate that low-grade adipose tissue inflammation is essential for a healthy adipose tissue expansion 297,335 . Whether inflammation is the trigger or result of obesity-associated defects remains an important area for future human studies.

\section{Prevention and treatment strategies for obesity and related cardiometabolic diseases}

In the treatment of obesity, and indirectly its related cardiometabolic risks, creating a negative energy balance is imperative. The most common strategies to achieve weight loss include decrease in energy intake, increase in energy expenditure or a combination between both ${ }^{336}$. Lifestyle interventions including combined diet and physical activity have been shown to be effective in the prevention of diabetes, reducing diabetes incidence by $47-57 \%$ over a 3-6 year timeframe 337,338 . 
Combined caloric restriction and physical activity interventions have been shown to be superior to diet-only intervention in terms of (in particular) weight management after weight loss and weight loss per se, as indicated in several meta-analyses ${ }^{339-341}$, even in the initial-onset phase (during the first 6 months) 342. Physical activity interventions without caloric restriction, including several types of interventions, such as endurance exercise, walking interventions, resistance training or habitual activity (number of steps throughout the day), have been shown to induce weight reductions (depending on the exercise modalities used), although to a lower extent (2-3\%) as compared to combined treatments ${ }^{343-347}$. However, endurance exercise is also associated with other health benefits like improvements in blood lipid profiles, insulin sensitivity and a reduction in inflammatory markers as recently reviewed by Ross et al. ${ }^{348}$. In this type of interventions, the intensity of the exercise seems to play an important role in the magnitude of weight loss and in improving metabolic profile ${ }^{349}$. Weight loss is assumed to include both loss of fat mass and fat-free mass. However, a restricted reduction in fat-free mass is associated with a lesser reduction in resting energy expenditure ${ }^{350,351}$. Therefore, the use of resistance training in addition to combined intervention might be important to preserve or potentially increase fat-free mass ${ }^{352}$, thereby also improving glycemic control and insulin sensitivity (independent of changes in BMI) 353,354 and other risk markers for cardiometabolic health ${ }^{355}$. Recently, high-intensity interval training was shown to have positive effects on glucose control and cardiometabolic health in populations at risk of cardiac complications and metabolic disease ${ }^{356}$. In short, combined lifestyle interventions (including diet plus exercise) as well as endurance exercise interventions of moderate- to highintensity (with or without resistance training) may help individuals with obesity to achieve a clinically relevant weight loss (3-5\% of initial body weight ${ }^{357}$ ), improve weight maintenance and cardiometabolic risk profile.

Despite the effectiveness of dietary and lifestyle modifications in the treatment of obesity and its related complications, these lifestyle changes are difficult to maintain and weight regain is typically found. Therefore, several anti-obesity and antidiabetic treatments have been developed, which are approved by the US Food and Drug Administration (FDA), while many other potential therapies are currently being investigated ${ }^{358}$. Currently, five FDA-approved anti-obesity drugs 
are on the market (ADA obesity treatment, 2016), which act centrally (Saxenda, Belviq, Qsymia and Mysimba) ${ }^{359-363}$ or peripherally (Orlistat) ${ }^{364}$.

Of special interest, to promote insulin sensitivity, inhibition of adipose tissue lipolysis might be an attractive therapeutic strategy in obese individuals. In this regard, preclinical studies in mouse models revealed that partial inhibition adipose tissue lipolysis protects against IR without affecting body weight via increased de novo lipogenesis 265,365. However, in clinical research, no pharmacological lipase (ATGL or HSL) inhibitors have been used so far. Nicotinic acid is a recognized lipid lowering agent able to bind GPR109A ${ }^{366-368}$, a $G_{i}$ coupled receptor responsible for partial inhibition of adipose tissue lipolysis 217,218, which results in reduced circulating FFA concentrations and an increased plasma glucose clearance in humans ${ }^{369-371}$. However, chronic treatment with nicotinic acid might even raise plasma FFA levels, thereby deteriorating glucose metabolism and insulin sensitivity 371,372 . Moreover, an analog of nicotinic acid, acipimox, has a more favorable profile on insulin sensitivity via decreased circulating FFA and an increased glucose clearance as shown in obese diabetic and non-diabetic individuals 373,374 . Despite some contrasting results showing decreased 375-377, increased 378,379 or even unaffected 380 plasma FFA concentrations, chronic acipimox treatment has been reported to moderately improve glycemic control and reduce glycated hemoglobin levels in non-insulin dependent diabetic individuals ${ }^{378}$, increase insulin signaling in skeletal muscle of obese insulin resistant individuals ${ }^{377}$ and decrease hepatic glucose production in obese and T2DM individuals ${ }^{375-377 .}$

\section{Outline of this thesis}

This thesis focuses on the endocrine regulation of adipose tissue lipid mobilization and the impact of exercise intervention in obese individuals, with a specific emphasis on the natriuretic peptide system and in particular atrial natriuretic peptide (ANP). The main goal of the current thesis was to gain more insight into the endocrine regulation of lipid mobilization in human obesity by combining cross-sectional and human clinical studies, together with ex vivo and in situ studies directed at understanding the underlying molecular mechanisms. 


\section{Aim 1: To determine the role of adipocyte ANP-mediated lipolysis in different adipose tissue depots under metabolically compromised conditions}

First, Chapter 2 provides a comprehensive review on the role of natriuretic peptides in the control of lipid metabolism and insulin sensitivity. This review provides data on the natriuretic peptide system, its signaling mechanisms, as well as its associations with cardiometabolic diseases. In particular, the metabolic roles of natriuretic peptides in adipose tissue, skeletal muscle and liver function are discussed in relation to the control of insulin sensitivity. Finally, human studies modulating the natriuretic peptide system by exercise or exercise intervention are implemented in this overview. In light of the growing body of evidence regarding the importance of ANP in stimulating adipose tissue lipid mobilization, it remains unknown whether this effect is comparable between adipose tissue depots, especially in distinct metabolic phenotypes. Accordingly, in Chapter 3, an unique design enabled us to investigate ANP-mediated lipid mobilization ex vivo in isolated subcutaneous and visceral mature adipocytes of lean, obese and obese type 2 diabetic individuals. Additionally, the molecular ANP signaling pathway was examined in more detail in these individuals.

\section{Aim 2: To assess the relative contributions of adipose tissue dysfunction characteristics to whole-body insulin resistance}

Different determinants of adipose tissue dysfunction contribute to the development of insulin resistance, but the relative contributions of an impaired adipose tissue lipid metabolism and inflammation remains to be elucidated. Chapter 4 provides novel insights into the associations between subcutaneous and visceral adipose tissue morphology, lipid mobilization, inflammation and whole-body insulin resistance in a cohort of lean and obese individuals with or without type 2 diabetes. For this study, analyses on ex vivo lipolysis and adipose tissue morphology were combined with a detailed quantification of adipose tissue immune cell populations using state-of-the-art methodology (flow cytometry) in paired subcutaneous and visceral adipose tissue samples. 


\section{Aim 3: To gain insight in the physiological regulation of adipose tissue lipid mobilization and the effects of non-pharmacological and pharmacological intervention in obese insulin resistant men}

Further extending on our outcome of the study outlined in Chapter 3, where we studied ex vivo ANP-mediated lipolysis in insulin resistance, the relevance of subcutaneous lipid mobilization under local adrenergic blockade during acute exercise was examined in Chapter $\mathbf{5}$ in an in situ setting in lean and obese individuals, as well as the potential effects of exercise intervention on the subcutaneous adipose tissue lipid mobilization were studied. More specifically, we explored ANP-mediated subcutaneous lipid mobilization during an acute exercise bout in lean individuals and obese insulin sensitive or insulin resistant individuals, by using the golden standard microdialysis technique. In addition, systemic responses with respect to whole-body lipid mobilization were assessed. Furthermore, in a training intervention study, the effects of 12 week supervised exercise training on ANP-mediated lipid mobilization in situ and whole-body lipid mobilization were assessed in obese insulin resistant individuals.

Besides non-pharmacological treatment strategies, preclinical studies suggest that partial inhibition of adipose tissue lipolysis may be a promising strategy to alleviate insulin resistance in obese pre-diabetic people. In Chapter 6, the clinical impact of pharmacological inhibition of adipose tissue lipid mobilization, using acipimox, during moderate-intense endurance-type exercise on postprandial glycemic control was examined in males obese type 2 diabetic patients.

Finally, in Chapter 7, the main findings from these studies described in this thesis are discussed in a broader perspective and suggestions for future research are provided. 


\section{References}

1. World Health Organization. http://www.who/int/mediacentre/factssheets/fs311/en/, updated June 2016

2. Ng M, Fleming T, Robinson M, Thomson B, Graetz N, Margono C et al (2014) Global, regional and national prevalence of overweight and obesity in children and adults during 1980-2013: a systematic analysis for the Global Burden of Disease Study 2013. Lancet 384:766-781

3. Blundell J, Baker J, Boyland E, Blaak E, Charzewska J, de Henauw S et al (2017) Variations in the prevalence of obesity among European countries, and a consideration of possible causes. Obes Facts 10:25-37

4. Luke A, Cooper R (2013) Physical activity does not influence obesity risk: time to clarify the public health message. Int J Epidemiol 42:1831-1836

5. Blair S, Archer E, Hand G (2013) Commentary: Luke and Cooper are wrong: physical activity has a crucial role in weight management and determinants of obesity. Int J Epidemiol 42:1836-1838

6. Hill J, Peters J (2013) Commentary: Physical activity and weight control. Int J Epidemiol 42:1840-1842

7. Swinburn B, Sacks G, Hall K, McPherson K, Finegood D, Moodie M, Gotmaker S (2011) The global obesity pandemic: shaped by global drivers and local environments. Lancet 378:804-814

8. Flegal K, Kit B, Orpana H, Graubardi B (2013) Association of all-cause mortality with overweight and obesity using standard body mass index categories: a systematic review and meta-analysis. JAMA 30:71-82

9. Curtis J, Selter J, Wang Y, Rathore S, Jovin I, Jadbabaie F et al (2005) The obesity paradox: body mass index and outcomes in patients with heart failure. Arch Intern Med 165:55-61

10. Huxley R, Mendis S, Zheleznyakov E, Reddy S, Chan J (2010) Body mass index, waist circumference and waist:hip ratio as predictors of cardiovascular risk--a review of the literature. Eur J Clin Nutr 64:16-22

11. Khan S, Hull R, Utzschneider K (2006) Mechanisms linking obesity to insulin resistance and type 2 diabetes. Nature 444:840-846

12. Emerging Risk Factors C, Wormser D, Kaptoge S, Di Angelantonio E, Wood A, Pennells L et al (2011) Separate and combined associations of body-mass index and abdominal adiposity with cardiovascular disease: collaborative analysis of 58 prospective studies. Lancet 377:1085-1095

13. Preiss K, Brennan L, Clarke D (2013) A systematic review of variables associated with the relationship between obesity and depression. Obes Rev 14:906-918

14. Bhaskaran K, Douglas I, Forbes H, dos Santos Silva I, Leon D, Smeeth L (2014) Body-mass index and risk of 22 specific cancers: a population-based cohort study of 5.24 million UK adults. Lancet 384:755-765

15. Renegan A, Zwahlen M, Egger M (2015) Adiposity and cancer risk: new mechanistic insights from epidemiology. Nat Rev Cancer 15:484-498

16. Guh D, Zhang W, Bansback N, Amarsi Z, Laird Birmingham C, Anis A (2009) The incidence of co-morbidities related to obesity and overweight: a systematic review and meta-analysis. BMC Public Health 9:88

17. Shah N, Roux F (2009) The relationship of obesity and obstructive sleep apnea. Clin Chest Med 30:455-465

18. Forhan M, Gill S (2013) Obesity, functional mobility and quality of life. Best practice \& research Clinical endocrinology \& metabolism 27:129-137 
19. Whitlock G, Lewington S, Sherliker P, Clarke R, Emberson J, Halsey J et al (2009) Body-mass index and cause-specific mortality in 900,000 adults: collaborative analyses of 57 prospective studies. Lancet 373:1083-1096

20. Wang Y, McPherson K, Marsh T, Gortmaker S, Brown M (2011) Health and economic burden of the projected obesity trends in the USA and the UK. Lancet 378:815-825

21. Guilherme A, Virbasius J, Puri V, Czech M (2008) Adipocyte dysfunction linking obesity to insulin resistance and type 2 diabetes. Nat Rev Meol Cell Biol 9:367-377

22. Shulman G (2014) Ectopic fat in insulin resistance, dyslipidemia and cardiometabolic disease. N Engl J Med 371:1131-1141

23. Perry R, Samuel V, Petersen K, Shulman G (2014) The role of hepatic lipids in hepatic insulin resistance and type 2 diabetes. Nature 510:84-91

24. Samuel V, Petersen K, Shulman G (2010) Lipid-induced insulin resistance: unravelling the mechanism. Lancet 375:2267-2277

25. Olefsky J, Glass C (2010) Macrophages, inflammation, and insulin resistance. Annu Rev Physiol 72:219-246

26. Stinkens R, Goossens G, Jocken J, Blaak E (2015) Targeting fatty acid metabolism to improve glucose metabolism. Obes Rev 16:715-57

27. Cooke A, Connaughton R, Lyons C, McMorrow A, Roche H (2016) Fatty acids and chronic low grade inflammation associated with obesity and the metabolic syndrome. Eur J Pharmacol 785:207-214

28. Farese R, Standaert M, Ishizuka T, Yu B, Arnold T, Cooper D (1991) The role of diacylglycerol/protein kinase $C$ signaling in insulin-stimulated glucose transport. Trans Assoc Am Physicians 104:187-193

29. Yu C, Chen Y, Cline G, Zhang D, Zong H, Wang Y et al (2002) Mechanism by which fatty acids inhibit insulin activation of insulin receptor substrate-1 (IRS-1)-associated phosphatidylinositol 3-kinase activity in muscle. J Biol Chem 277:50230-50236

30. Goossens G (2008) The role of adipose tissue dysfunction in the pathogenesis of obesity-related insulin resistance. Physiol Behav 94:206-218

31. Samuel V, Shulman G (2016) The pathogenesis of insulin resistance: integrating signaling pathways and substrate flux. J Clin Invest 126:12-22

32. Samuel V, Shulman G (2012) Mechanisms for insulin resistance: common threads and missing links. Cell 148:852-871

33. WHO Global report on Diabetes 2016

34. Abdul-Ghani M, Jenkinson C, Richardson D, Tripathy D, De Fronzo R (2006) Insulin secretion and action in subjects with impaired fasting glucose and impaired glucose tolerance: results from the Veterans Administration Genetic Epidemiology Study. Diabetes 55:1430-1435

35. Bock G, Chittilapilly E, Basu R, Toffolo G, Cobelli C, Chandramouli V et al (2007) Contribution of hepatic and extrahepatic insulin resistance to the pathogenesis of impaired fasting glucose: role of increased rates of gluconeogenesis. Diabetes $56: 1703-1711$

36. Meyer C, Pimenta W, Woerle H, Van Haeften T, Szoke E, Mitrakou A, Gerich J (2006) Different mechanisms for impaired fasting glucose and impaired postprandial glucose tolerance in humans. Diabetes Care 29:1909-1914

37. Blaak E (2017) Characterisation of fatty acids metabolism in different insulinresistant phenotypes by means of stable isotopes. Proc Nutr Soc 19:1-7

38. Krotkiewski M, Björntorp $P$, Sjöström L, Smith U (1983) Impact of obesity on metabolism in men and women. Importance of regional adipose tissue distribution. J Clin Invest 72:1150-1162 
39. Lundgren M, Svensson M, Lindmark S, Renström F, Ruge T, Eriksson J (2007) Fat cell enlargement is an independent marker of insulin resistance and hyperleptinaemia. Diabetologia 50:625-633

40. Potts J, Coppack S, Fisher R, Humphreys S, Gibbons G, Frayn K (1995) Impaired postprandial clearance of triacylglycerol-rich lipoproteins in adipose tissue in obese subjects. Am J Physiol 268:E588-E594

41. Goldberg I, Eckel R, Abumrad N (2009) Regulation of fatty acid uptake into tissues: lipoprotein lipase- and CD36-mediated pathways. J Lipid Res 50:S86-S90

42. Jocken J, Blaak E (2008) Catecholamine-induced lipolysis in adipose tissue and skeletal muscle in obesity. Physiol Behav 94:219-230

43. Nellemann B, Gormsen L, Sorensen L, Christiansen J, Nielsen S (2012) Impaired insulin-mediated antilipolysis and lactate release in adipose tissue of upper-body obese women. Obesity 20:57-64

44. Blaak E, van Baak M, Kemerink G, Pakbiers M, Heidendal G, Saris W (1995) Betaadrenergic stimulation and abdominal subcutaneous fat blood flow in lean, obese, and reduced-obese subjects. Metabolism 44:183-187

45. Goossens GH, Bizzarri A, Venteclef N, Essers Y, Cleutjens J, Konings E et al (2011) Increased adipose tissue oxygen tension in obese compared with lean men is accompanied by insulin resistance, impaired adipose tissue capillarization, and inflammation. Circulation 124:67-76

46. Virtue S, Vidal-Puig A (2010) Adipose tissue expandability, lipotoxicity and the Metabolic Syndrome--an allostatic perspective. Biochim Biophys Acta 1801:338-349

47. Taylor R, Holman R (2015) Normal weight individuals who develop type 2 diabetes: the personal fat threshold. Clin Sci 128:405-410

48. Jocken J, Goossens G, Boon H, Mason R, Essers Y, Havekes B et al (2013) Insulinmediated suppression of lipolysis in adipose tissue and skeletal muscle of obese type 2 diabetic men and men with normal glucose tolerance. Diabetologia 56:2255-2265

49. Hulver M, Berggren J, Carper M, Miyazaki M, Ntambi J, Hoffman E et al (2005) Elevated stearoyl-CoA desaturase-1 expression in skeletal muscle contributes to abnormal fatty acid partitioning in obese humans. Cell Metab 2:251-261

50. Bergman B, Perreault L, Hunerdosse D, Koehler M, Samek A, Eckel R (2009) Intramuscular lipid metabolism in the insulin resistance of smoking. Diabetes 58:2220-2227

51. Perreault L, Bergman B, Hunerdosse D, Eckel R (2010) Altered intramuscular lipid metabolism relates to diminished insulin action in men, but not women, in progression to diabetes. Obesity 18:2093-2100

52. Bergman B, Hunerdosse D, Kerege A, Playdon M, Perreault L (2012) Localisation and composition of skeletal muscle diacylglycerol predicts insulin resistance in humans. Diabetologia 55:1140-1150

53. Timmers S, de Vogel-van den Bosch J, Hesselink M, van Beurden D, Schaart G, Ferraz $M$ et al (2011) Paradoxical increase in TAG and DAG content parallel the insulin sensitizing effect of unilateral DGAT1 overexpression in rat skeletal muscle. PLoS One 6:e14503

54. Hotamisligil G, Shargil N, Spiegelman B (1993) Adipose expression of tumor necrosis factor-alpha: direct role in obesity-linked insulin resistance. Science 259:150-156

55. Makki K, Froguel P, Wolowczuk I (2013) Adipose tissue in obesity-related inflammation and insulin resistance: cells, cytokines, and chemokines. ISRN Inflamm 2013:139239

56. Bai Y, Sun Q (2015) Macrophage recruitment in obese adipose tissue. Obes Rev $16: 127-136$ 
57. Weisberg S, McCann D, Desai M, Rosenbaum M, Leibel R, Ferrante A (2003) Obesity is associated with macrophage accumulation in adipose tissue. J Clin Invest 112:1796-1808

58. Wynn T, Chawla A, Pollard J (2013) Macrophage biology in development, homeostasis and disease. Nature 496:445-455

59. Mraz M, Haluzik M (2014) The role of adipose tissue immune cells in obesity and lowgrade inflammation. J Endocrinol 222:R113-R127

60. Sica A, Mantovani A (2012) Macrophage plasticity and polarization: in vivo veritas. J Clin Invest 122:787-795

61. Kratz M, Coats B, Hisert K, Hagman D, Mutskov V, Peris E et al (2014) Metabolic dysfunction drives a mechanistically distinct proinflammatory phenotype in adipose tissue macrophages. Cell Metab 20:614-625

62. Goossens G, Blaak E, Theunissen R, Duijvestijn A, Clément K, Tervaert J, Thewissen $M$ (2012) Expression of NLRP3 inflammasome and $T$ cell population markers in adipose tissue are associated with insulin resistance and impaired glucose metabolism in humans. Mol Immunol 50:142-149

63. Machann J, Häring $H$, Schick F, Stumvoll M (2004) Intramyocellular lipids and insulin resistance. Diabetes Obes Metab 6:239-248

64. Glass C, Olefsky J (2012) Inflammation and lipid signaling in the etiology of insulin resistance. Cell Metab 15:635-645

65. Ritter O, Jelenik T, Roden M (2015) Lipid-mediated muscle insulin resistance: different fat, different pathways. J Mol Med 93:831-843

66. Blaak E, Hul G, Verdich C, Stich V, Martinez A, Petersen M et al (2006) Fat oxidation before and after a high fat load in the obese insulin-resistant state. J Clin Endocrinol Metab 91:1462-1469

67. Koves T, Ussher J, Noland R, Slentz D, Mosedale M, Ilkayeva O et al (2008) Mitochondrial overload and incomplete fatty acid oxidation contribute to skeletal muscle insulin resistance. Cell Metab 7:45-56

68. Blaak E, van Aggel-Leijssen D, Wagenmakers A, Saris W, van Baak M (2000) Impaired oxidation of plasma-derived fatty acids in type 2 diabetic subjects during moderate-intensity exercise. Diabetes 49:2102-2107

69. Giacca A, Xiao C, Oprescu A, Carpentier A, Lewis G (2011) Lipid-induced pancreatic $\beta$-cell dysfunction: focus on in vivo studies. Am J Physiol Endocrinol Metab 300:E255E262

70. Poitout V, Amyot J, Semache M, Zarrouki B, Hagman D, Fontés G (2010) Glucolipotoxicity of the pancreatic beta cell. Biochim Biophys Acta 1801:289-298

71. Donnelly K, Smith C, Schwarzenberg S, Jessurun J, Boldt M, Parks E (2005) Sources of fatty acids stored in liver and secreted via lipoproteins in patients with nonalcoholic fatty liver disease. J Clin Invest 115:1343-1351

72. Ratziu V, Giral P, Charlotte F, Bruckert E, Thibault V, Theodorou I et al (2000) Liver fibrosis in overweight patients. Gastroenterology 118:1117-1123

73. Bellentani S, Saccoccio G, Masutti F, Crocè L, Brandi G, Sasso F et al (2000) Prevalence of and risk factors for hepatic steatosis in Nothern Italy. Ann Intern Med 132:112-117

74. Fabbrini E, Sullivan S, Klein S (2010) Obesity and nonalcoholic fatty liver disease: biochemical, metabolic and clinical implications. Hepatology 51:679-689

75. McGavock J, Victor R, Unger R, Szczepaniak L, American College of Physicians and the American Physiological Society (2006) Adiposity of the heart, revisited. Ann Intern Med 144:517-524 
76. Iacobellis G, Corradi D, Sharma A (2005) Epicardial adipose tissue: anatomic, biomolecular and clinical relationships with the heart. Nat Clin Pract Cardiovasc Med 2:536-543

77. Harris K, Kassis A, Major G, Chou C (2012) Is the gut microbiota a new factor contributing to obesity and its metabolic disorders? J Obes 2012:879151

78. Murphy K, Bloom S (2006) Gut hormones and the regulation of energy homeostasis. Nature 444:854-859

79. Diamant M, Blaak E, de Vos W (2011) Do nutrient-gut-microbiota interactions play a role in human obesity, insulin resistance and type 2 diabetes? Obes Rev 12:272-281

80. Delzenne N, Cani P, Everard A, Neyrinck A, Bindels L (2015) Gut microorganisms as promising targets for the management of type 2 diabetes. Diabetologia 58:22062217

81. Bäckhed F, Manchester J, Semenkovich C, Gordon J (2007) Mechanisms underlying the resistance to diet-induced obesity in germ-free mice. Proc Natl Acad Sci USA 104:979-984

82. Barlow G, Yu A, Mathur R (2015) Role of the Gut Microbiome in Obesity and Diabetes Mellitus. Nutr Clin Pract 30:787-797

83. Cox M, Jackson J, Stanton M, Rojas-Triana A, Bober L, Laverty M et al (2009) Shortchain fatty acids act as antiinflammatory mediators by regulating prostaglandin $E(2)$ and cytokines. World J Gastroenterol 15:5549-5557

84. Jones M, Ganopolsky J, Martoni C, Labbé A, Prakash S (2014) Emerging science of the human microbiome. Gut Microbes 5:446-457

85. Reijnders D, Goossens G, Hermes G, Neis E, van der Beek C, Most J et al (2016) Effects of Gut Microbiota Manipulation by Antibiotics on Host Metabolism in Obese Humans: A Randomized Double-Blind Placebo-Controlled Trial. Cell Metab 24:341

86. Aberdein N, Schweizer M, Bali D (2014) Sodium acetate decreases phosphorylation of hormone sensitive lipase in isoproterenol-stimulated 3T3-L1 mature adipocytes. Adipocyte 3:121-125

87. Ge H, Lix X, Weiszmann J, Wang P, Baribault H, Chen J et al (2008) Activation of G protein-coupled receptor 43 in adipocytes leads to inhibition of lipolysis and suppression of plasma free fatty acids. Endocrinology 149:4519-4526

88. Canfora E, Jocken J, Blaak E (2015) Short-chain fatty acids in control of body weight and insulin sensitivity. Nat Rev Endocrinol 11:577-591

89. Dijk W, Kersten S (2014) Regulation of lipoprotein lipase by Angptl4. Trends Endocrinol Metab 25:146-155

90. Alex S, Lichtenstein L, Dijk W, Mensink R, Tan N, Kersten S (2014) ANGPTL4 is produced by entero-endocrine cells in the human intestinal tract. Histochem Cell Biol 141:383-391

91. Brands M, Sauerwein H, Ackermans M, Kersten S, Serlie M (2013) Omega-3 longchain fatty acids strongly induce angiopoietin-like 4 in humans. J Lipid Res 54:615621

92. Ruge $T$, Sukonina V, Kroupa O, Makoveichuk E, Lundgren M, Svensson $M$ et al (2012) Effects of hyperinsulinemia on lipoprotein lipase, angiopoietin-like protein 4, and glycosylphosphatidylinositol-anchored high-density lipoprotein binding protein 1 in subjects with and without type 2 diabetes mellitus. Metabolism 61:652-660

93. Kersten S, Lichtenstein L, Steenbergen E, Mudde K, Hendriks $H$, Hesselink M et al (2009) Caloric restriction and exercise increase plasma ANGPTL4 levels in humans via elevated free fatty acids. Arterioscler Thromb Vasc Biol 29:969-974 
94. Van Raalte D, Brands M, Serlie M, Mudde K, Stienstra R, Sauerwein H et al (2012) Angiopoietin-like protein 4 is differentially regulated by glucocorticoids and insulin in vitro and in vivo in healthy humans. Exp Clin Endocrinol Diabetes 120:598-603

95. Zlobine I, Gopal K, Ussher J (2016) Lipotoxicity in obesity and diabetes-related cardiac dysfunction. Biochim Biophys Acta 1860:1555-1568

96. Collins S (2014) A heart-adipose tissue connection in the regulation of energy metabolism. Nat Rev Endocrinol 10:157-163

97. Volpe M (2014) Natriuretic peptides and cardio-renal disease. In J Cardiol 176:630639

98. Schlueter N, de Sterke A, Willmes D, Spranger J, Jordan J, Birkenfeld A (2014) Metabolic actions of natriuretic peptides and therapeutic potential in the metabolic syndrome. Pharmacol Ther 144:12-27

99. Coué M, Moro C (2016) Natriuretic peptide control of energy balance and glucose homeostasis. Biochimie 124:84-91

100. Moro C (2016) Targeting cardiac natriuretic peptides in the therapy of diabetes and obesity. Expert Opin Ther Targets 20:1445-1452

101. Shen W, Wang Z, Punyanita M, Lei J, Sinav A, Kral J et al (2003) Adipose tissue quantification by imaging methods: a proposed classification. Obes Res 11:5-16

102. Karpe F, Pinnick K (2015) Biology of upper-body and lower-body adipose tissue-link to whole-body phenotypes. Nat Rev Endocrinol 11:90-100

103. Tchkonia T, Thomou T, Zhu Y, Karagiannides I, Pothoulakis C, Jensen M, Kirkland J (2013) Mechanisms and metabolic implications of regional differences among fat depots. Cell Metab 17:644-656

104. Ibrahim M (2010) Subcutaneous and visceral adipose tissue: structural and functional differences. Obes Rev 11:11-18

105. Fox C, Massaro J, Hoffmann U, Pou K, Maurovich-Horvat P, Liu C et al (2007) Abdominal visceral and subcutaneous adipose tissue compartments: association with metabolic risk factors in the Framingham Heart Study. Circulation 116:39-48

106. Mundi M, Karpyak M, Koutsari C, Votruba S, O'Brien P, Jensen M (2010) Body fat distribution, adipocyte size, and metabolic characteristics of nondiabetic adults. J Clin Endocrinol Metab 95:67-73

107. Marinou K, Hodson L, Vasan S, Fielding B, Banerjee R, Brismar K et al (2014) Structural and functional properties of deep abdominal subcutaneous adipose tissue explain its association with insulin resistance and cardiovascular risk in men. Diabetes Care 37:821-829

108. Casey B, Kohrt W, Schwartz R, Van Pelt R (2014) Subcutaneous adipose tissue insulin resistance is associated with visceral adiposity in postmenopausal women. Obesity 22:1458-1463

109. Jensen M, Sarr M, Dumesic D, Southorn P, Levine J (2003) Regional uptake of meal fatty acids in humans. Am J Physiol Endocrinol Metab 285:E1282-E1288

110. Vortruba S, Mattison R, Dumesic D, Koutsari C, Jensen M (2007) Meal fatty acid uptake in visceral fat in women. Diabetes 56:2589-2597

111. Björntorp P (1990) Portal adipose tissue as a generator of risk factors for cardiovascular disease and diabetes. Arteriosclerosis 10:493-496

112. Jensen $M$ (2006) Is visceral fat involved in the pathogenesis of the metabolic syndrome? Human model. Obesity 14 Suppl 1:20S-24S

113. Rosen E, Spiegelman B (2014) What we talk about when we talk about fat. Cell $156: 20-44$ 
114. Knittle J, Timmers K, Ginsberg-Fellner F, Brown R, Katz D (1979) The growth of adipose tissue in children and adolescents. Cross-sectional and longitudinal studies of adipose cell number and size. J Clin Invest 63:239-246

115. Moreno-Indias I, Tinahones F (2015) Impaired adipose tissue expandability and lipogenic capacities as ones of the main causes of metabolic disorders. J Diabetes Res 2015:970375

116. Spalding K, Arner E, Westermark P, Bernard S, Buchholz BA, Bergmann O et al (2008) Dynamics of fat cell turnover in humans. Nature 453:783-787

117. Arner P, Bernard S, Salehpour M, Possnert G, Liebl J, Steier P et al (2011) Dynamics of human adipose lipid turnover in health and metabolic disease. Nature 478:110113

118. Faust I, Johnson P, Stern J, Hirsch J (1978) Diet-induced adipocyte number increase in adult rats: a new model of obesity. Am J Physiol 235:E279-286

119. Tang Q, Lane M (2012) Adipogenesis: from stem cell to adipocyte. Annu Rev Biochem 81:715-736

120. Arner E, Westermark P, Spalding K, Britton T, Rydén M, Frisén J et al (2010) Adipocyte turnover: relevance to human adipose tissue morphology. Diabetes 59:105-109

121. Srdic B, Stokic E, Korac A, Ukropina M, Velickovic K, Breberina M (2010) Morphological characteristics of abdominal adipose tissue in normal-weight and obese women of different metabolic profiles. Exp Clin Endocrinol Diabetes 118:713718

122. Lönn M, Mehlig K, Bengtsson C, Lissner L (2010) Adipocyte size predicts incidence of type 2 diabetes in women. FASEB J 24:326-331

123. Drolet R, Richard C, Sniderman A, Mailloux J, Fortier M, Huot C et al (2008) Hypertrophy and hyperplasia of abdominal adipose tissues in women. Int $\mathrm{J}$ Obes 32:283-291

124. Frayn K (2002) Adipose tissue as a buffer for daily lipid flux. Diabetologia 45:12011210

125. Danforth E (2000) Failure of adipocyte differentiation causes type II diabetes mellitus? Comment on: In vivo modulation of Hmgic reduces obesity. Nat Genet $26: 13$

126. Rydén M, Andersson D, Bernard S, Spalding K, Arner P (2013) Adipocyte triglyceride turnover and lipolysis in lean and overweight subjects. J Lipid Res 54:2909-2913

127. Acosta J, Douagi I, Andersson D, Bäckdahl J, Rydén M, Arner P, Laurencikiene J (2016) Increased fat cell size: a major phenotype of subcutaneous white adipose tissue in non-obese individuals with type 2 diabetes. Diabetologia 59:560-570

128. Weyer C, Foley J, Bogardus C, Tataranni P, Pratley R (2000) Enlarged subcutaneous abdominal adipocyte size, but not obesity itself, predicts type II diabetes independent of insulin resistance. Diabetologia 43:1498-1506

129. Eriksson J, Smith U, Waagstein F, Wysocki M, Jansson P (1999) Glucose turnover and adipose tissue lipolysis are insulin-resistant in healthy relatives of type 2 diabetes patients: is cellular insulin resistance a secondary phenomenon. Diabetes 48:1572-1578

130. Fang L, Guo F, Zhou L, Stahl R, Grams J (2015) The cell size and distribution of adipocytes from subcutaneous and visceral fat is associated with type 2 diabetes mellitus in humans. Adipocyte 5:273-279

131. Yang J, Eliasson B, Smith U, Cushman S, Sherman A (2012) The size of large adipose cells is a predictor of insulin resistance in first-degree relatives of type 2 diabetic patients. Obesity 20:932-938 
132. Laforest S, Labrecque J, Michaud A, Cianflone K, Tchernof A (2015) Adipocyte size as a determinant of metabolic disease and adipose tissue dysfunction. Crit Rev Clin Lab Sci 52:301-313

133. Dubois S, Heilbronn L, Smith R, Albu J, Kelley D, Ravussin E, Look AHEAD Adipose Research Group (2006) Decreased expression of adipogenic genes in obese subjects with type 2 diabetes. Obesity 14:1543-1552

134. Pasarica M, Xie H, Hymel D, Bray D, Greenway F, Ravussin E, Smith S (2009) Lower total adipocyte number but no evidence for small adipocyte depletion in patients with type 2 diabetes. Diabetes Care 32:900-902

135. Van Tienen F, van der Kallen C, Lindsey P, Wanders R, van Greevenbroek M, Smeets H (2011) Preadipocytes of type 2 diabetes subjects display an intrinsic gene expression profile of decreased differentiation capacity. Int J Obes 35:1154-1164

136. Andersson D, Eriksson Hogling D, Thorell A, Toft E, Qvisth V, Näslund E et al (2014) Changes in subcutaneous fat cell volume and insulin sensitivity after weight loss. Diabetes Care 37:1831-1836

137. Rydén M, Andersson D, Bergström I, Arner P (2014) Adipose tissue and metabolic alterations: regional differences in fat cell size and number matter, but differently: a cross-sectional study. J Clin Endocrinol Metab 99:E1870-E1876

138. Kim S, Lun M, Wang M, Senyo S, Guillermier C, Patwari P, Steinhauser M (2014) Loss of White Adipose Hyperplastic Potential Is Associated with Enhanced Susceptibility to Insulin Resistance. Cell Metab 20:1049-1058

139. Zimmerman R, Straus J, Haemmerle G, Schoiswohl G, Birner-Gruenberger R, Riederer $M$ et al (2004) Fat mobilization in adipose tissue is promoted by adipose triglyceride lipase. Science 306:1383-1386

140. Lafontan M, Langin D (2009) Lipolysis and lipid mobilization in human adipose tissue. Progr Lipid Res 48:275-297

141. Nielsen T, Jessen N, Jorgensen J, Moller N, Lund S (2014) Dissecting adipose tissue lipolysis: molecular regulation and implications for metabolic disease. J Mol Endocrinol 52:R199-R222

142. Zouhal H, Jacob C, Delamarche P, Grata-Delamarche A (2008) Catecholamines and the effects of exercise, training and gender. Sports Med 38:401-423

143. Fairn J, Garcia-Sainz J (1983) Adrenergic regulation of adipocyte metabolism. J Lipid Res 24:945-966

144. Pecquery R, Leneveu M, Giudicelli Y (1983) Characterization of the beta-adrenergic receptors of hamster white fat cells. Evidence against an important role for the alpha 2-receptor subtype in the adrenergic control of lipolysis. Biochim Biophys Acta 731:397-405

145. Bartness T, Liu Y, Shrestha Y, Ryu V (2014) Neural innervation of white adipose tissue and the control of lipolysis. Front Neuroendocrinol 35:473-493

146. Robidoux J, Martin T, Collins S (2004) Beta-adrenergic receptors and regulation of energy expenditure: a family affair. Annu Rev Pharmacol Toxicol 44:297-323

147. Mauriège $P$, Després $J$, Preud'homme $D$, Pouliot $M$, Marcottte $M$, Tremblay $A$, Bouchard C (1991) Regional variation in adipose tissue lipolysis in lean and obese men. J Lipid Res 32:1625-1633

148. Barbe P, Millet L, Galitzky J, Lafontan M, Berlan M (1996) In situ assessment of the role of the beta 1 -, beta 2 - and beta 3-adrenoceptors in the control of lipolysis and nutritive blood flow in human subcutaneous adipose tissue. $\mathrm{Br} \mathrm{J}$ Pharmacol 117:907913 
149. Tavernier G, Barbe P, Galitzky J, Berlan M, Caput D, Lafontan M, Langin D (1996) Expression of beta3-adrenoceptors with low lipolytic action in human subcutaneous white adipocytes. J Lipid Res 37:87-97

150. Blaak E, Saris W, van Baak M (1993) Adrenoceptor subtypes mediating catecholamine-induced thermogenesis in man. Int J Obes Relat Metab Disord 17 Suppl3:S78-S82

151. De Matteis R, Arch J, Petroni M, Ferrari D, Cinti S, Stock M (2002) Immunohistochemical identification of the beta(3)-adrenoceptor in intact human adipocytes and ventricular myocardium: effect of obesity and treatment with ephedrine and caffeine. In J Obes Relat Metab Disord 26:1442-1450

152. Gomez-Ambrosi J, Frühbeck G, Aguado M, Milagro F, MArgareto J, Martinez A (2001) Divergent effects of an alpha2-adrenergic antagonist on lipolysis and thermogenesis: interactions with a beta3-adrenergic agonist in rats. Int J Mol Med 8:103-109

153. Mottillo E, Balasubramanian P, Lee Y, Weng C, Kershaw E, Granneman J (2014) Coupling of lipolysis and de novo lipogenesis in brown, beige, and white adipose tissues during chronic $\beta 3$-adrenergic receptor activation. J Lipid Res 55:2276-2286

154. Buemann B, Toubro S, Astrup A (2000) Effects of the two beta3-agonists, ZD7114 and ZD2079 on 24 hour energy expenditure and respiratory quotient in obese subjects. Int J Obes Relat Metab Disord 24:1553-1560

155. Schiffelers S, Blaak E, Saris W, van Baak M (2000) In vivo beta3-adrenergic stimulation of human thermogenesis and lipid use. Clin Pharmacol Ther 67:558-566

156. Redman L, de Jonge L, Fang X, Gamlin B, Recker D, Greenway F et al (2007) Lack of an effect of a novel beta3-adrenoceptor agonist, TAK-677, on energy metabolism in obese individuals: a double-blind, placebo-controlled randomized study. J Clin Endocrinol Metab 92:527-531

157. Cypess A, Weiner L, Roberts-Toler C, Franquet E, Kessler S, Kahn P et al (2015) Activation of human brown adipose tissue by a $\beta 3$-adrenergic receptor agonist. Cell Metab 21:33-38

158. Lafontan M, Berlan M (1993) Fat cell adrenergic receptors and the control of white and brown fat cell function. J Lipid Res 34:1057-1091

159. Langin D (2006) Control of fatty acid and glycerol release in adipose tissue lipolysis. C R Biol 329:597-607

160. Greenberg A, Egan J, Wek S, Garty N, Blanchette-Mackie E, Londos C (1991) Perilipin, a major hormonally regulated adipocyte-specific phosphoprotein associated with the periphery of lipid storage droplets. J Biol Chem 266:11341-11346

161. Stralfors $P$, Björgell $P$, Belfrage $P$ (1984) Hormonal regulation of hormone-sensitive lipase in intact adipocytes: identification of phosphorylated sites and effects on the phosphorylation by lipolytic hormones and insulin. Proc Natl Acad Sci USA 81:33173321

162. Garton A, Campbell D, Cohen P, Yeaman S (1988) Primary structure of the site on bovine hormone-sensitive lipase phosphorylated by cyclic AMP-dependent protein kinase. FEBS Lett 229:68-72

163. Anthonsen M, Rönnstrand L, Wernstedt C, Degerman E, Holm C (1998) Identification of novel phosphorylation sites in hormone-sensitive lipase that are phosphorylated in response to isoproterenol and govern activation properties in vitro. J Biol Chem 273:215-221

164. Egan J, Greenberg A, Chang M, Wek S, Moos M, Londos C (1992) Mechanism of hormone-stimulated lipolysis in adipocytes: translocation of hormone-sensitive lipase to the lipid storage droplet. Proc Natl Acad Sci USA 89:8537-8541 
165. Shen W, Patel S, Miyoshi H, Greenberg A, Kraemer F (2009) Functional interaction of hormone-sensitive lipase and perilipin in lipolysis. J Lipid Res 50:2306-2313

166. Wang H, Hu L, Dalen K, Dorward H, Marcinikiewicz A, Russell D et al (2009) Activation of hormone-sensitive lipase requires two steps, protein phosphorylation and binding to the PAT-1 domain of lipid droplet coat proteins. J Biol Chem 284:32116-32125

167. Lass A, Zimmermann R, Haemmerle G, Riederer M, Schoiswohl G, Schweiger M et al (2006) Adipose triglyceride lipase-mediated lipolysis of cellular fat stores is activated by CGI-58 and defective in Chanarin-Dorfman Syndrome. Cell Metab 3:309-319

168. Granneman J, Moore H, Krishnamoorthy R, Rathod M (2009) Perilipin controls lipolysis by regulating the interactions of $A B$-hydrolase containing 5 (Abhd5) and adipose triglyceride lipase. J Biol Chem 284:34538-34544

169. McGrath M, de Bold M, de Bold A (2005) The endocrine function of the heart. Trends Endocrinol Metab 16:469-477

170. Clerico A, Giannoni A, Vittorini S, Passino C (2011) Thirty years of the heart as an endocrine organ: physiological role and clinical utility of cardiac natriuretic hormones. Am J Physiol Heart Circ Physiol 301:H12-H20

171. Sengenes C, Berlan M, De Glisezinski I, Lafontan M, Galitzky J (2000) Natriuretic peptides: a new lipolytic pathway in human adipocytes. FASEB J 14:1345-1351

172. Sengenes C, Bouloumie A, Hauner H, Berlan M, Busse R, Lafontan M et al (2003) Involvement of a cGMP-dependent pathway in the natriuretic peptide-mediated hormone-sensitive lipase phosphorylation in human adipocytes. J Biol Chem 278:48617-48626

173. Birkenfeld A, Boschmann M, Moro C, Adams F, Heusser K, Franke G et al (2005) Lipid mobilization with physiological atrial natriuretic peptide concentrations in humans. J Clin Endocrinol Metab 90:3622-3628

174. Bordicchia M, Ceresiani M, Pavani M, Minardi D, Polito M, Wabitsch M et al (2016) Insulin/glucose induces natriuretic peptide clearance receptor in human adipocytes: a metabolic link with the cardiac natriuretic pathway. Am J Physiol Regul Integr Comp Physiol 311:R104-R114

175. Galitzky J, Sengenes C, Thalamas C, Marques M, Senard J, Lafontan M, Berlan M (2001) The lipid-mobilizing effect of atrial natriuretic peptide is unrelated to sympathetic nervous system activation or obesity in young men. J Lipid Res 42:536544

176. Birkenfeld A, Boschmann M, Moro C, Adams F, Heusser K, Tank J et al (2006) Betaadrenergic and Atrial Natriuretic Peptide Interactions on Human Cardiovascular and Metabolic Regulation. J Clin Endocrinol Metab 91:5069-5075

177. Moro C, Galitzky J, Sengenes C, Crampes F, Lafontan M, Berlan M (2004) Functional and pharmacological characterization of the natriuretic peptide-dependent lipolytic pathway in human fat cells. J Pharmacol Exp Therp 308:984-992

178. Moro C, Klimcakova E, Lafontan M, Berlan M, Galitzky J (2007) Phosphodiesterase-5A and neutral endopeptidase activities in human adipocytes do not control atrial natriuretic peptide-mediated lipolysis. Br J Pharmacol 152:1102-1110

179. Armani A, Marzolla V, Rosano G, Fabbri A, Caprio M (2011) Phosphodiesterase type 5 (PDE5) in the adipocyte: a novel player in fat metabolism. Trends Endocrinol Metab 22:404-411

180. Aversa A, Caprio M, Antelmi A, Armani A, Brama M, Greco E et al (2011) Exposure to phosphodiesterase type 5 inhibitors stimulates aromatase expression in human adipocytes in vitro. J Sex Med 8:696-704 
181. Nagase M, Katafuchi T, Hirose S, Fujita T (1997) Tissue distribution and localization of natriuretic peptide receptor subtypes in stroke-prone spontaneously hypertensive rats. J Hypertens 15:1235-1243

182. Matsukawa N, Grzesik W, Takahashi N, Pandey K, Pang S, Yamauchi M, Smithies O (1999) The natriuretic peptide clearance receptor locally modulates the physiological effects of the natriuretic peptide system. Proc Natl Acad Sci USA 96:7403-7408

183. Pivovarova O, Gögebakan Ö, Klöting N, Sparwasser A, Weickert M, Haddad I et al (2012) Insulin up-regulates natriuretic peptide clearance receptor expression in the subcutaneous fat depot in obese subjects: a missing link between CVD risk and obesity. J Clin Endocrinol Metab 94:E731-E739

184. Pandey K (1992) Kinetic analysis of internalization, recycling and redistribution of atrial natriuretic factor-receptor complex in cultured vascular smooth-muscle cells. Ligand-dependent receptor down-regulation. Biochem J 288:55-61

185. Sarzani R, Paci V, Zingaretti C, Pierleoni C, Cinti S, Cola G et al (1995) Fasting inhibits natriuretic peptides clearance receptor expression in rat adipose tissue. J Hypertens 13:1241-1246

186. Miyashita K, Itoh H, Tsujimoto H, Tamura N, Fukunaga Y, Sone M et al (2009) Natriuretic peptides/cGMP/cGMP-dependent protein kinase cascades promote muscle mitochondrial biogenesis and prevent obesity. Diabetes 58:2880-2892

187. Nakatsuji H, Maeda N, Hibuse T, Hiuge A, Hirata A, Kuroda $Y$ et al (2010) Reciprocal regulation of natriuretic peptide receptors by insulin in adipose cells. Biochem Biophys Res Comm 392:100-105

188. Collins S, Sarzani R, Bordicchia M (2014) Coordinate control of adipose 'browning' and energy expenditure by $\beta$-adrenergic and natriuretic peptide signalling. Int J Obes Suppl 4:S17-S20

189. Moller L, Dalman L, Norrelund H, Billestrup N, Frystyk J, Moller N, Jorgensen J (2009) Impact of fasting on growth hormone signaling and action in muscle and fat. J Clin Endocrinol Metab 94:965-972

190. Yip R, Goodman H (1999) Growth Hormone and Dexamethasone Stimulate Lipolysis and Activate Adenylyl Cyclase in Rat Adipocytes by Selectively Shifting Gia2 to Lower Density Membrane Fractions. Endocrinology 140:1219-1227

191. Gravholt C, Schmitz O, Simonsen L, Büllow J, Christiansen J, Moller N (1999) Effects of a physiological $\mathrm{GH}$ pulse on interstitial glycerol in abdominal and femoral adipose tissue. Am J Physiol 277:E848-E854

192. Samra J, Clark M, Humphreys S, MacDonald I, Bannister P, Matthews D, Frayn K (1999) Suppression of the nocturnal rise in growth hormone reduces subsequent lipolysis in subcutaneous adipose tissue. Eur J Clin Invest 29:1045-1052

193. Sakharova A, Horowitz J, Surya S, Goldenberg N, Harber M, Symons K, Barkan A (2008) Role of growth hormone in regulating lipolysis, proteolysis, and hepatic glucose production during fasting. J Clin Endocrinol Metab 93:2755-2759

194. Djurhuus C, Gravholt C, Nielsen S, Mengel A, Christiansen J, Schmitz O, Moller N (2002) Effects of cortisol on lipolysis and regional interstitial glycerol levels in humans. Am J Physiol Endocrinol Metab 283:E172-E177

195. Samra J, Clark M, Humphreys S, MacDonald I, Bannister P, Frayn K (1998) Effects of physiological hypercortisolemia on the regulation of lipolysis in subcutaneous adipose tissue. J Clin Endocrinol Metab 83:626-631

196. Ottosson M, Lönnroth P, Björntorp P, Edén S (2000) Effects of cortisol and growth hormone on lipolysis in human adipose tissue. J Clin Endocrinol Metab 85:799-803

197. Obregon M (2014) Adipose tissues and thyroid hormones. Front Physiol 5:479 
198. Taniguchi A, Kataoka K, Kono T, Oseko F, Okuda H, Nagata I, Imura H (1987) Parathyroid hormone-induced lipolysis in human adipose tissue. J Lipid Res 28:490494

199. Bousquet-Mélou A, Galitzky J, Lafontan M, Berlan M (1995) Control of lipolysis in intra-abdominal fat cells of nonhuman primates: comparison with humans. J Lipid Res 36:451-461

200. Xu F, De Pergola G, Björntorp P (1991) Testosterone increases lipolysis and the number of beta-adrenoceptors in male rat adipocytes. Endocrinology 128:379-382

201. Dicker A, Rydén M, Näslund E, Muehlen I, Wirén M, Lafontan M, Arner P (2004) Effect of testosterone on lipolysis in human pre-adipocytes from different fat depots. Diabetologia 47:420-428

202. Arner $P$ (2005) Effects of testosterone on fat cell lipolysis. Species differences and possible role in polycystic ovarian syndrome. Biochimie 87:39-43

203. Zang $H$, Rydén $M$, Wahlen $K$, Dahlman-Wright $K$, Arner $P$, Lindén Hirschberg $A$ (2007) Effects of testosterone and estrogen treatment on lipolysis signaling pathways in subcutaneous adipose tissue of postmenopausal women. Fertil Steril 88:100-106

204. Fan W, Yanase T, Nomura M, Okabe T, Goto K, Sato T et al (2005) Androgen receptor null male mice develop late-onset obesity caused by decreased energy expenditure and lipolytic activity but show normal insulin sensitivity with high adiponectin secretion. Diabetes 54:1000-1008

205. Bertin E, Arner P, Bolinder J, Hagström-Toft E (2001) Action of glucagon and glucagon-like peptide-1-(7-36) amide on lipolysis in human subcutaneous adipose tissue and skeletal muscle in vivo. J Clin Endocrinol Metab 86:1229-1234

206. Graveholt C, Moller N, Jensen M, Christiansen J, Schmitz O (2001) Physiological levels of glucagon do not influence lipolysis in abdominal adipose tissue as assessed by microdialysis. J Clin Endocrinol Metab 86:2085-2089

207. Frühbeck G, Méndez-Giménez L, Fernandez-Formoso J, Fernandez S, Rodriguez A (2014) Regulation of adipocyte lipolysis. Nutr Res Rev 27:63-93

208. Zhang Y, Goldman S, Baerga R, Zhao Y, Komatsu M, Jin S (2009) Adipose-specific deletion of autophagy-related gene 7 (atg7) in mice reveals a role in adipogenesis. Proc Natl Acad Sci USA 106:19860-19865

209. Lizaso A, Tan $K$, Lee $Y$ (2013) $\beta$-adrenergic receptor-stimulated lipolysis requires the RAB7-mediated autolysosomal lipid degradation. Autophagy 9:1228-1243

210. Singh R, Xiang Y, Wang Y, Baikati K, Cuervo A, Luu Y et al (2009) Autophagy regulates adipose mass and differentiation in mice. J Clin Invest 119:3329-3339

211. Cingolani F, Czaja M (2016) Regulation and Functions of Autophagic Lipolysis. Trends Endocrinol Metab 27:696-705

212. Jocken J, Goossens G, Blaak E (2014) Targeting adipose tissue lipid metabolism to improve glucose metabolism in cardiometabolic disease. EMJ Diabet 2:73-82

213. Rocchi A, He C (2015) Emerging roles of autophagy in metabolism and metabolic disorders. Front Biol 10:154-164

214. White M (1998) The IRS-signalling system: a network of docking proteins that mediate insulin action. Mol Cell Biochem 182:3-11

215. Carpenter C, Duckworth B, Auger K, Cohen B, Schaffhausen B, Cantley L (1990) Purification and characterization of phosphoinositide 3-kinase from rat liver. J Biol Chem 265:19704-19711

216. Choi Y, Park S, Hockman S, Zmuda-Trzebiatowska E, Svennelid F, Haluzik M et al (2006) Alterations in regulation of energy homeostasis in cyclic nucleotide phosphodiesterase 3B-null mice. J Clin Invest 116:3240-3251 
217. Ahmed K, Tunaru S, Tang C, Müller M, Gille A, Sassmann A et al (2010) An autocrine lactate loop mediates insulin-dependent inhibition of lipolysis through GPR81. Cell Metab 11:311-319

218. Taggart A, Kero J, Gan X, Cai T, Cheng K, Ippolito M et al (2005) (D)-betaHydroxybutyrate inhibits adipocyte lipolysis via the nicotinic acid receptor PUMA-G. J Biol Chem 280:26649-26652

219. Cai T, Ren N, Jin L, Cheng K, Kash S, Chen R et al (2008) Role of GPR81 in lactatemediated reduction of adipose lipolysis. Biochem Biophys Res Commun 377:987-991

220. Liu C, Wu J, Zhu J, Kuei C, Yu J, Shelton J et al (2009) Lactate inhibits lipolysis in fat cells through activation of an orphan G-protein-coupled receptor, GPR81. J Biol Chem 284:2811-2822

221. Sakurai T, Davenport R, Stafford S, Grosse J, Ogawa K, Cameron J et al (2014) Identification of a novel GPR81-selective agonist that suppresses lipolysis in mice without cutaneous flushing. Eur J Pharmacol 727:1-7

222. Langin D (2010) Adipose Tissue Lipolysis Revisited (Again!): Lactate Involvement in Insulin Antilipolytic Action. Cell Metab 11:242-243

223. Al-Lahham S, Poelofsen H, Priebe M, Weening D, Dijkstra M, Hoek A et al (2010) Regulation of adipokine

production in human adipose tissue by propionic acid. Eur J Clin Invest 40:401-407

224. Brown A, Goldsworthy S, Barnes A, Eilert M, Tcheang L, Daniels D et al. The Orphan G protein-coupled receptors GPR41 and GPR43 are activated by propionate and other short chain carboxylic acids. J Biol Chem 278:11312-11319

225. Le Poul E, Loison C, Struyf S, Springael J, Lannoy V et al (2003) Functional characterization of human receptors for short chain fatty acids and their role in polymorphonuclear cell activation. J Biol Chem 278:25481-25489

226. Ge H, Li X, Weiszmann J, Wang P, Baribault H, Chen J et al (2008) Activation of G protein-coupled receptor 43 in adipocytes leads to inhibition of lipolysis and suppression of plasma free fatty acids. Endocrinology 149:4519-4526

227. Rumberger J, Arch J, Green A (2014) Butyrate and other short-chain fatty acids increase the rate of lipolysis in 3T3-L1 adipocytes. Peer] 2:e611

228. Arner P (1995) Differences in lipolysis between human subcutaneous and omental adipose tissues. Ann Med 27:435-438

229. Wang Y, Sullivan S, Trujillo M, Lee M, Schneider S, Brolin R et al (2003) Perilipin expression in human adipose tissues: effects of severe obesity, gender, and depot. Obes Res 11:930-936

230. Fried S, Leibel R, Edens N, Kral J (1993) Lipolysis in intraabdominal adipose tissues of obese women and men. Obes Res 1:443-448

231. Rebuffé-Scrive M, Anderson B, Olbe L, Björntorp P (1990) Metabolism of adipose tissue in intraabdominal depots in severely obese men and women. Metabolism 39:1021-1025

232. Tchernof A, Bélanger C, Morisset A, Richard C, Mailloux J, Laberge $P$, Dupont $P$ (2006) Regional differences in adipose tissue metabolism in women: minor effect of obesity and body fat distribution. Diabetes 55:1353-1360

233. Boivin A, Brochu G, Marceau S, Marceau P, Hould F, Tchernof A (2007) Regional differences in adipose tissue metabolism in obese men. Metabolism 56:533-540

234. Leibel RL and Hirsch J (1987) Site- and sex-related differences in adrenoreceptor status of human adipose tissue. J Clin Endocrinol Metab 64:1205-1210

235. Lönnqvist $F$, Thörne A, Large V, Arner $P$ (1997) Sex differences in visceral fat lipolysis and metabolic complications of obesity. Arterioscler Thromb Vasc Biol $17: 1472-1480$ 
236. Meek S, Nair K, Jensen M (1999) Insulin regulation of regional free fatty acid metabolism. Diabetes 48:10-14

237. Jensen $M$ (1995) Gender differences in regional fatty acid metabolism before and after meal ingestion. J Clin Invest 96:2297-2303

238. Lee $M, \mathrm{Wu}$ Y, Fried $\mathrm{S}$ (2013) Adipose tissue heterogeneity: implication of depot differences in adipose tissue for obesity complications. Mol Aspects Med 34:1-11

239. Manolopoulos K, Karpe F, Frayn K (2012) Marked resistance of femoral adipose tissue blood flow and lipolysis to adrenaline in vivo. Diabetologia 55:3029-3037

240. Johnson J, Fried S, Pi-Sunyer F, Albu J (2001) Impaired insulin action in subcutaneous adipocytes from women with visceral obesity. Am J Physiol Endocrinol Metab 280:E40-E49

241. Fried S, Tittelbacj T, Blumenthal J, Sreenivasan U, Robey L, Yi J et al (2010) Resistance to the antilipolytic effect of insulin in adipocytes of African-American compared to Caucasian postmenopausal women. J Lipid Res 51:1193-1200

242. Rydén $M$, Backdahl J, Petrus $P$, Thorell A, Gao H, Coue $M$ et al (2016) Impaired atrial natriuretic peptide-mediated lipolysis in obesity. Int J Obes 40:714-20

243. Kovacova Z, Tharp W, Liu D, Wei W, Xie H, Collins S et al (2016) Adipose tissue natriuretic peptide receptor expression is related to insulin sensitivity in obesity and diabetes. Obesity 24:820-8

244. Mairal A, Langin D, Arner P, Hoffstedt J (2006) Human adipose triglyceride lipase (PNPLA2) is not regulated by obesity and exhibits low in vitro triglyceride hydrolase activity. Diabetologia 49:1629-1636

245. Berndt J, Kralisch S, Klöting N, Ruschke K, Kern M, Fasshauer M et al (2008) Adipose triglyceride lipase gene expression in human visceral obesity. Exp Clin Endocrinol Diabetes 116:203-210

246. Steinberg G, Kemp B, Watt M (2007) Adipocyte triglyceride lipase expression in human obesity. Am J Physiol Endocrinol Metab 293:E958-E964

247. Reynisdottir S, Dauzats M, Thörne A, Langin D (1997) Comparison of hormonesensitive lipase activity in visceral and subcutaneous human adipose tissue. J Clin Endocrinol Metab 82:4162-4166

248. Montague C, Prins J, Sanders L, Zhang J, Sewter C, Digby J et al (1998) Depotrelated gene expression in human subcutaneous and omental adipocytes. Diabetes 47:1384-1391

249. Lefebvre A, Laville M, Vega N, Riou J, van Gaal L, Auwerx J, Vidal H (1998) Depotspecific differences in adipose tissue gene expression in lean and obese subjects. Diabetes 47:98-103

250. Large V, Reynisdottir S, Langin D, Fredby K, Klannemark M, Holm C, Arner P (1999) Decreased expression and function of adipocyte hormone-sensitive lipase in subcutaneous fat cells of obese subjects. J Lipid Res 40:2059-2066

251. Lundgren M, Burén J, Lindgren P, Myrnäs T, Ruge T, Eriksson J (2008) Sex- and depot-specific lipolysis regulation in human adipocytes: interplay between adrenergic stimulation and glucocorticoids. Hormo Metab Res 40:854-860

252. Laurencikiene J, Skurk T, Kulyté A, Hedén P, Aström G, Sjölin E et al (2011) Regulation of lipolysis in small and large fat cells of the same subject. J Clin Endocrinol Metab 96:E2045-E2049

253. Bickel P, Tansey J, Welte M (2009) PAT proteins, an ancient family of lipid droplet proteins that regulate cellular lipid stores. Biochim Biophys Acta 1791:419-440

254. Ray H, Pinteur C, Frering V, Beylot M, Large V (2009) Depot-specific differences in perilipin and hormone-sensitive lipase expression in lean and obese. Lipids Health Dis $8: 58$ 
255. Tinahones F, Garrido-Sanchez L, Miranda M, Garcia-Almeida J, Macias-Gonzalez M, Ceperuelo $V$ et al (2010) Obesity and insulin resistance-related changes in the expression of lipogenic and lipolytic genes in morbidly obese subjects. Obes Surg 20:1559-1567

256. Moreno-Navarette J, Ortega F, Serrano M, Rodriguez-Hermosa J, Ricart W, Mingrone G, Fernandez-Real J (2014) CIDEC/FSP27 and PLIN1 gene expression run in parallel to mitochondrial genes in human adipose tissue, both increasing after weight loss. Int J Obes 38:865-872

257. Opie $L$ and Walfish $P$ (1963) Plasma free fatty acid concentrations in obesity. N Engl J Med 268:757-760

258. Karpe F, Dickmann J, Frayn K (2011) Fatty acids, obesity, and insulin resistance: time for a reevaluation. Diabetes 60:2441-2449

259. Langin D, Dicker A, Tavernier G, Hoffstedt J, Mairal A, Rydén M et al (2005) Adipocyte lipases and defect of lipolysis in human obesity. Diabetes 54:3190-3197

260. Large V, Arner P (1998) Regulation of lipolysis in humans. Pathophysiological modulation in obesity, diabetes, and hyperlipidaemia. Diabetes Metab 24:409-418

261. McQuaid S, Hodson L, Neville M, Dennis A, Cheeseman J, Humphreys S et al (2011) Downregulation of adipose tissue fatty acid trafficking in obesity: a driver for ectopic fat deposition. Diabetes 60:47-55

262. Jocken J, Goossens G, van Hees A, Frayn K, van Baak M, Stegen J et al (2008) Effect of beta-adrenergic stimulation on whole-body and abdominal subcutaneous adipose tissue lipolysis in lean and obese men. Diabetologia 51:320-327

263. Horowitz J, Coppack S, Paramore D, Cryer P, Zhao G, Klein S (1999) Effect of shortterm fasting on lipid kinetics in lean and obese women. Am J Physiol 276:E278-E284

264. Mittendorfer B, Magkos F, Fabbrini E, Mohammed B, Klein S (2009) Relationship between body fat mass and free fatty acid kinetics in men and women. Obesity 17:1872-1877

265. Girousse A, Tavernier G, Valle C, Moro C, Mejhert N, Dinel A et al (2013) Partial inhibition of adipose tissue lipolysis improves glucose metabolism and insulin sensitivity without alteration of fat mass. PLoS Biol 11:e1001485

266. Campbell P, Carlson M, Nurjhan N (1994) Fat metabolism in human obesity. Am J Physiol 266:E600-E605

267. Roust L, Jensen M (1993) Postprandial free fatty acid kinetics are abnormal in upper body obesity. Diabetes 42:1567-1573

268. Bugianesi E, Gastaldelli A, Vanni E, Gambino R, Cassader M, Baldi S et al (2005) Insulin resistance in non-diabetic patients with non-alcoholic fatty liver disease: sites and mechanisms. Diabetologia 48:634-642

269. Gastaldelli A, Cusi K, Pettiti M, Hardies J, Miyazaki Y, Berria R et al (2007) Relationship between hepatic/visceral fat and hepatic insulin resistance in nondiabetic and type 2 diabetic subjects. Gastroenterology 133:496-506

270. Lomonaco R, Ortiz-Lopez C, Orsak B, Webb A, Hardies J, Darland C et al (2012) Effect of adipose tissue insulin resistance on metabolic parameters and liver histology in obese patients with nonalcoholic fatty liver disease. Hepatology 55:1389-1397

271. Armstrong MJ, Hazlehurst JM, Hull D, Guo K, Borrows S, Yu J et al (2014) Abdominal subcutaneous adipose tissue insulin resistance and lipolysis in patients with nonalcoholic steatohepatitis. Diabetes Obes Metab 16:651-660

272. Bell LN, Wang J, Muralidharan S, Chalasani S, Fullenkamp AM, Wilson LA et al (2012) Relationship between adipose tissue insulin resistance and liver histology in nonalcoholic steatohepatitis: a pioglitazone versus vitamin E versus placebo for the 
treatment of nondiabetic patients with nonalcoholic steatohepatitis trial follow-up study. Hepatology 56:1311-1318

273. Gastaldelli A, Gaggini M, DeFronzo R (2017) Role of Adipose Tissue Insulin Resistance in the Natural History of T2DM: Results from the San Antonio Metabolism Study. Diabetes doi: 10.2337/db16-1167. [Epub ahead of print]

274. Jacobsson B, Smith U (1972) Effect of cell size on lipolysis and antilipolytic action of insulin in human fat cells. J Lipid Res 13:651-656

275. Blaak E, Van Baak M, Kemerink G, Pakbiers M, Heidendal G, Saris W (1994) Beta adrenergic stimulation of energy expenditure and forearm skeletal muscle metabolism in lean and obese men. Am J Physiol 267: E306-E315

276. Blaak E, Van Baak M, Kemerink G, Pakbiers M, Heidendal G, Saris W (1994) Betaadrenergic stimulation of skeletal muscle metabolism in relation to weight reduction in obese men. Am J Physiol 267: E316-E322

277. Connacher A, Bennet W, Jung R, Bier D, Smith C, Scrimgeour C, Rennie M (1991) Effect of adrenaline infusion on fatty acid and glucose turnover in lean and obese human subjects in the post-absorptive and fed states. Clin Sci 81:635-644

278. Horowitz J, Klein S (2000) Whole body and abdominal lipolytic sensitivity to epinephrine is suppressed in upper body obese women. Am J Physiol Endocrinol Metab 278:E1144-E1152

279. Jensen M (1997) Lipolysis: contribution from regional fat. Annu Rev Nutr 17:127-139

280. Webber J, Taylor J, Greathead H, Dawson J, Buttery P, Macdonald I (1994) A comparison of the thermogenic, metabolic and haemodynamic responses to infused adrenaline in lean and obese subjects. Int J Obes Relat Metab Disord 18:717-724

281. Reynisdottir S, Wahrenberg H, Carlstrom K, Rossner S, Arner P (1994) Catecholamine resistance in fat cells of women with upper-body obesity due to decreased expression of beta 2-adrenoceptors. Diabetologia 37:428-435

282. Wajchenberg B, Giannella-Neto D, da Silva M, Santos R (2002) Depot-specific hormonal characteristics of subcutaneous and visceral adipose tissue and their relation to the metabolic syndrome. Horm Metab Res 34:616-621

283. Arner P (2005) Human fat cell lipolysis: biochemistry, regulation and clinical role. Best Pract Res Clin Endocrinol Metab 19:471-482

284. Rydén M, Jocken J, van Harmelen V, Dicker A, Hoffstedt J, Wirén M et al (2007) Comparative studies of the role of hormone-sensitive lipase and adipose triglyceride lipase in human fat cell lipolysis. Am J Physiol Endocrinol Metab 292:E1847-E18455

285. Andersson D, Löfgren P, Thorell A, Arner P, Hoffstedt J (2011) Visceral fat cell lipolysis and cardiovascular risk factors in obesity. Horm Metab Res 43:809-815

286. Borsheim $E$, Lönnroth $P$, Knardahl S, Jansson $P$ (2000) No difference in the lipolytic response to beta-adrenoceptor stimulation in situ but a delayed increase in adipose tissue blood flow in moderately obese compared with lean men in the postexercise period. Metabolism 49:579-587

287. Schiffelers S, Akkermans J, Saris W, Blaak E (2003) Lipolytic and nutritive blood flow response to beta-adrenoceptor stimulation in situ in subcutaneous abdominal adipose tissue in obese men. Int J Obes Relat Metab Disord 27:227-231

288. Bougnères $P$, Stunff $C$, Pecqueur $C$, Pinglier $E$, Adnot $P$, Ricquier D (1997) In vivo resistance of lipolysis to epinephrine. A new feature of childhood onset obesity. J Clin Invest 99:2568-2573

289. Enoksson S, Talbot M, Rife F, Tamborlane W, Sherwin R, Caprio S (2000) Impaired in vivo stimulation of lipolysis in adipose tissue by selective beta2-adrenergic agonist in obese adolescent girls. Diabetes 49:2149-2153 
290. Hellström L, Langin D, Reynisdottir S, Dauzats M, Arner P (1996) Adipocyte lipolysis in normal weight subjects with obesity among first-degree relatives. Diabetologia 39:921-928

291. Dicker A, Aström G, Wahlén K, Hoffstedt J, Näslund E, Wirén M et al (2009) Primary differences in lipolysis between human omental and subcutaneous adipose tissue observed using in vitro differentiated adipocytes. Horm Metab Res 41:350-355

292. Arora P, Wu C, Khan A, Bloch D, Davis-Dusenbery B, Ghorbani A et al (2013) Atrial natriuretic peptide is negatively regulated by microRNA-425. J Clin Invest 123:33783382

293. Hellman B, Larsson S, Westman S (1963) Mast cell content and fatty acid metabolism in the epididymal fat pad of obese mice. Acta Physiol Scand 58:255-262

294. Hotamisligil G, Shargill N, Spiegelman B (1993) Adipose expression of tumor necrosis factor-alpha: direct role in obesity-linked insulin resistance. Science 259:87-91

295. Weisberg S, McCann D, Desai M, Rosenbaum M, Leibel R, Ferrante A (2003) Obesity is associated with macrophage accumulation in adipose tissue. J Clin Invest 112:1796-1808

296. Xu H, Barnes G, Yang Q, Tan G, Yang D, Chou C et al (2003) Chronic inflammation in fat plays a crucial role in the development of obesity-related insulin resistance. J Clin Invest 112:1821-1830

297. Cinti S, Mitchell G, Barbatelli G, Murano I, Ceresi E, Faloia E et al (2005) Adipocyte death defines macrophage localization and function in adipose tissue of obese mice and humans. J Lipid Res 46:2347-2355

298. Ouchi N, Parker J, Lugus J, Walsh K (2011) Adipokines in inflammation and metabolic disease. Nat Rev Immunol 11:85-97

299. Brestoff J, Artis D (2015) Immune regulation of metabolic homeostasis in health and disease. Cell 161:146-160

300. Shoelson S, Lee J, Goldfine A (2006) Inflammation and insulin resistance. J Clin Invest 116:1793-1801

301. Haase J, Weyer U, Immig K, Klöting N, Blüher M, Eilers J et al (2014) Local proliferation of macrophages in adipose tissue during obesity-induced inflammation. Diabetologia 57:562-571

302. Osborn O and Olefsky J (2012) The cellular and signaling networks linking the immune system and metabolism in disease. Nat Med 18:363-374

303. Mathis D (2013) Immunological goings-on in visceral adipose tissue. Cell Metab 17:851-859

304. Kosteli A, Sugaru E, Haemmerle G, Martin J, Lei J, Zechner R et al (2010) Weight loss and lipolysis promote a dynamic immune response in murine adipose tissue. J Clin Invest 120:3466-3479

305. Shi H, Kokoeva M, Inouye K, Tzameli I, Yin H, Flier J (2006) TLR4 links innate immunity and fatty acid-induced insulin resistance. J Clin Invest 116:3015-3025

306. Suganami T, Nishida J, Ogawa Y (2005) A paracrine loop between adipocytes and macrophages aggravates inflammatory changes: role of free fatty acids and tumor necrosis factor alpha. Arterioscler Thromb Vasc Biol 25:2062-2068

307. Nguyen M, Satoh H, Favelyukis S, Babendure J, Imamura T, Sbodio J et al (2005) JNK and tumor necrosis factor-alpha mediate free fatty acid-induced insulin resistance in 3T3-L1 adipocytes. J Biol Chem 280:35361-35371

308. Prieur X, Mok C, Velagapudi V, Nunez V, Fuentes L, Montaner D et al (2011) Differential lipid partitioning between adipocytes and tissue macrophages modulates macrophage lipotoxicity and M2/M1 polarization in obese mice. Diabetes 60:797-809 
309. Sell H, Habich C, Eckel J (2012) Adaptive immunity in obesity and insulin resistance. Nat Rev Endocrinol 8:709-716

310. Klein-Wieringa I, Andersen S, Kwekkeboom J, Giera M, de Lange-Brokaar B, van Osch G et al (2013) Adipocytes modulate the phenotype of human macrophages through secreted lipids. J Immunol 191:1356-1363

311. Kratz M, Coats B, Hisert K, Hagman D, Mutskov V, Peris E et al (2014) Metabolic dysfunction drives a mechanistically distinct proinflammatory phenotype in adipose tissue macrophages. Cell Metab 20:614-625

312. Castoldi A, Naffah de Souza C, Camara N, Moraes-Vieira P (2016) The Macrophage Switch in Obesity Development. Front Immunol 6:637

313. Oh D, Morinaga $H$, Talukdar S, Bae E, Olefsky J (2012) Increased macrophage migration into adipose tissue in obese mice. Diabetes 61:346-354

314. Chawla A, Nguyen K, Goh Y (2011) Macrophage-mediated inflammation in metabolic disease. Nat Rev Immunol 11:738-749

315. Lumeng C, Bodzin J, Saltiel A (2007) Obesity induces a phenotypic switch in adipose tissue macrophage polarization. J Clin Invest 117:175-184

316. Lumeng C, Deyoung S, Bodzin J, Saltiel A (2007) Increased inflammatory properties of adipose tissue macrophages recruited during diet-induced obesity. Diabetes 56:16-23

317. Rausch M, Weisberg S, Vardhana P, Tortoriello D (2008) Obesity in C57BL/6] mice is characterized by adipose tissue hypoxia and cytotoxic T-cell infiltration. Int J Obes 32:451-463

318. Winer S, Chan Y, Paltser G, Truong D, Tsui H, Bahrami J et al (2009) Normalization of obesity-associated insulin resistance through immunotherapy. Nat Med 15:921929

319. Lynch L, Nowak M, Varghese B, Clark J, Hogan A, Toxavidis V et al (2012) Adipose tissue invariant NKT cells protect against diet-induced obesity and metabolic disorder through regulatory cytokine production. Immunity 37:574-587

320. Winer D, Winer S, Shen L, Wadia P, Yantha J, Paltser G et al (2011) B lymphocytes promote insulin resistance through modulation of $T$ lymphocytes and production of pathogenic IgG antibody. Nat Med 17:610-617

321. DeFuria J, Belkina A, Jagannathan-Bogdan M, Snyder-Cappione J, Carr J, Nersesova $Y$ et al (2013) B cells promote inflammation in obesity and type 2 diabetes through regulation of $\mathrm{T}$ cell function and an inflammatory cytokine profile. Proc Natl Acad Sci USA 110:5133-5138

322. Lee B, Kim M, Pae M, Yamamoto Y, Eberlé D, Shimada T et al (2016) Adipose Natural Killer Cells Regulate Adipose Tissue Macrophages to Promote Insulin Resistance in Obesity. Cell Metab 23:685-698

323. Talukdar S, Oh D, Bandyopadhyay G, Li D, Xu J, McNelis J et al (2012) Neutrophils mediate insulin resistance in mice fed a high-fat diet through secreted elastase. Nat Med 18:1407-1412

324. Stefanovic-Racic M, Yang X, Turner M, Mantell B, Stolz D, Sumpter T et al (2012) Dendritic cells promote macrophage infiltration and comprise a substantial proportion of obesity-associated increases in CD11c+ cells in adipose tissue and liver. Diabetes $61: 2330-2339$

325. Liu J, Divoux A, Sun J, Zhang J, Clément K, Glickman J et al (2009) Genetic deficiency and pharmacological stabilization of mast cells reduce diet-induced obesity and diabetes in mice. Nat Med 15:940-945

326. Kintscher U, Hartge M, Hess K, Foryst-Ludwig A, Clemenz M, Wabitsch M et al (2008) T-lymphocyte infiltration in visceral adipose tissue: a primary event in adipose tissue 
inflammation and the development of obesity-mediated insulin resistance. Arterioscler Thromb Vasc Biol 28:1304-1310

327. Feuerer M, Herrero L, Cipolletta D, Naaz A, Wong J, Nayer A et al (2009) Lean, but not obese, fat is enriched for a unique population of regulatory $T$ cells that affect metabolic parameters. Nat Med 15:930-939

328. Ilan Y, Maron R, Tukpah A, Maioli T, Murugaiyan G et al (2010) Induction of regulatory $T$ cells decreases adipose inflammation and alleviates insulin resistance in ob/ob mice. Proc Natl Acad Sci USA 107:9765-9770

329. Eller K, Kirsch A, Wolf A, Sopper S, Tagwerker A, Stanzl U et al (2011) Potential role of regulatory $T$ cells in reversing obesity-linked insulin resistance and diabetic nephropathy. Diabetes 60:2954-2962

330. Lumeng $C$ and Saltiel A (2011) Inflammatory links between obesity and metabolic disease. J Clin Invest 121:2111-2117

331. Klöting N, Fasshauer M, Dietrich A, Kovacs P, Schön M, Kern M et al (2010) Insulinsensitive obesity. Am J Physiol Endocrinol Metab 299:E506-E515

332. Hardy O, Perugini R, Nicoloro S, Gallagher-Dorval K, Puri V, Straubhaar J, Czech M (2011) Body mass index-independent inflammation in omental adipose tissue associated with insulin resistance in morbid obesity. Surg Obes Relat Dis 7:60-67

333. Granneman J, Li P, Zhu Z, Lu Y (2005) Metabolic and cellular plasticity in white adipose tissue I: effects of beta3-adrenergic receptor activation. Am J Physiol Endocrinol Metab 289:E608-E616

334. Cao Y (2013) Angiogenesis and Vascular Functions in Modulation of Obesity, Adipose Metabolism, and

Insulin Sensitivity. Cell Metab 18:478-489

335. Blüher M (2016) Adipose tissue inflammation: a cause or consequence of obesityrelated insulin resistance Clin Sci 130:1603-1614

336. Spiegelman B, Flier J (2001) Obesity and the regulation of energy balance. Cell 104:531-543

337. Den Boer A, Herraets I, Stegen J, Roumen C, Corpeleijn E, Schaper N et al (2013) Prevention of the metabolic syndrome in IGT subjects in a lifestyle intervention: results from the SLIM study. Nutr Metab Cardiovasc Dis 23:1147-1153

338. Penn L, White M, Lindström J, den Boer A, Blaak E, Eriksson J et al (2013) Importance of weight loss maintenance and risk prediction in the prevention of type 2 diabetes: analysis of European Diabetes Prevention Study RCT. PLoS One 8:e57143

339. Franz M, VanWormer J, Crain A, Boucher J, Histon T, Caplan W et al (2007) Weightloss outcomes: a systematic review and meta-analysis of weight-loss clinical trials with a minimum 1-year follow-up. J Am Diet Assoc 107:1755-1767

340. Wu T, Gao X, Chen M, van Dam RM (2009) Long-term effectiveness of diet-plusexercise interventions vs. diet-only interventions for weight loss: a meta-analysis. Obes Rev 10:313-323

341. Foster-Schubert K, Alfano C, Duggan C, Xiao L, Campbell K, Kong A et al. (2012) Effect of diet and exercise, alone or combined, on weight and body composition in overweight-to-obese postmenopausal women. Obesity 20:1628-1638

342. Goodpaster B, Delany J, Otto A, Kuller L, Vockley J, South-Paul J et al. (2010) Effects of diet and physical activity interventions on weight loss and cardiometabolic risk factors in severely obese adults: a randomized trial. Jama 304:1795-1802

343. Thorogood A, Mottillo S, Shimony A, Filion K, Joseph L, Genest J et al (2011) Isolated aerobic exercise and weight loss: a systematic review and meta-analysis of randomized controlled trials. Am J Med 124: 747-755 
344. Alves J, Gale C, Mutrie N, Correia J, Batty G (2009) A 6-month exercise intervention among inactive and overweight favela-residing women in Brazil: the Caranguejo Exercise Trial. Am J Public Health 99:76-80

345. Nishijima H, Satake K, Igarashi K, Morita N, Kanazawa N, Okita K (2007) Effects of exercise in overweight Japanese with multiple cardiovascular risk factors. Med Sci Sports Exerc 39:926-933

346. Donnelly J, Honas J, Smith B, Mayo M, Gibson C, Sullivan D et al (2013) Aerobic exercise alone results in clinically significant weight loss for men and women: Midwest Exercise Trial 2. Obesity 21:E219-E228

347. Irwin M, Yasui Y, Ulrich C, Bowen D, Rudolph R, Schwartz R et al. (2003) Effect of exercise on total and intra-abdominal body fat in postmenopausal women: a randomized controlled trial. JAMA 289:323-330

348. Ross R, Blair S, de Lannoy L, Després JP, Lavie CJ (2015) Changing the endpoints for determining effective obesity management. Prog Cardiovasc Dis 57:330-336

349. Chin S, Kahathuduwa C, Binks M (2016) Physical activity and obesity: what we know and what we need to know. Obes Rev 17:1226-1244

350. Nelson K, Weinsier R, Long C, Schutz Y (1992) Prediction of resting energy expenditure from fat-free mass and fat mass. Am J Clin Nutr 56:848-856

351. Kirk E, Donnelly J, Smith B, Honas J, Lecheminant J, Bailey B et al (2009) Minimal resistance training

improves daily energy expenditure and fat oxidation. Med Sci Sports Exerc 41:11221129

352. Hunter G, Byrne N, Sirikul B, Fernandez J, Zuckerman P, Darnell B, Gower B (2008) Resistance training conserves fat-free mass and resting energy expenditure following weight loss. Obesity 16:1045-1051

353. Roberts C, Croymans D, Aziz N, Butch A, Lee C (2013) Resistance training increases SHBG in overweight/obese, young men. Metabolism 62:725-733

354. Moghadasi M, Abdollahpur N, Abdehgah E, Hosseini F, Ali S (2014) Effect of 8-week resistance training on adipocyte fatty acid binding protein in obese middle-aged men. Int Jl Sport Studies 4:1198-1204

355. Clark J and Goon D (2015) The role of resistance training for treatment of obesity related health issues and for changing health status of the individual who is overfat or obese: a review. J Sports Med Phys Fitness 55:205-222

356. Cassidy S, Thoma C, Houghton D, Trenell M (2017) High-intensity interval training: a review of its impact on glucose control and cardiometabolic health. Diabetologia 60:7-23

357. Jensen M, Ryan D, Apovian C, Ard J, Comuzzie A, Donato K et al. (2014) 2013 AHA/ACC/ TOS Guideline for the management of overweight and obesity in adults: a report of the American College of Cardiology/American Heart Association Task Force on Practice Guidelines and The Obesity Society. Circulation 129:S102-S138

358. Kusminski C, Bickel $P$, Scherer $P$ (2016) Targeting adipose tissue in the treatment of obesity-associated diabetes. Nat Rev Drug Discov 15:639-660

359. Astrup A, Carraro R, Finer N, Harper A, Kunesova M, Lean M et al (2012) Safety, tolerability and sustained weight loss over 2 years with the once-daily human GLP-1 analog, liraglutide. Int J Obes 36:843-854

360. Pi-Sunyer X, Astrup A, Fujioka K, Greenway F, Halpern A, Krempf M et al (2015) A Randomized, Controlled Trial of $3.0 \mathrm{mg}$ of Liraglutide in Weight Management. $\mathrm{N}$ Engl J Med 373:11-22 
361. Smith S, Weissman N, Anderson C, Sanchez M, Chuang E, Stubbe S et al (2010) Multicenter, placebo-controlled trial of larcaserin for weight management. N Engl J Med 363:245-256

362. Verrotti A, Scaparrotta A, Agostinelli S, Di Pillo S, Chiarelli F, Grosso S (2011) Topiramate-induced weight loss: a review. Epilepsy Res 95:189-199

363. Greenway F, Fujioka K, Plodkowski R, Mudaliar S, Guttadauria M, Erickson J et al (2010) Effect of naltrexone plus bupropion on weight loss in overweight and obese adults (COR-I): a multicentre, randomised, double-blind, placebo-controlled, phase 3 trial. Lancet 376:595-605

364. Sumithran P and Proietto J (2014) Benefit-risk assessment of orlistat in the treatment of obesity. Drug Saf 37:597-608

365. Morigny P, Houssier M, Mouisel E, Langin D (2016) Adipocyte lipolysis and insulin resistance. Biochimie 125:259-266

366. Soga T, Kamohara M, Takasaki J, Matsumoto S, Saito T, Ohishi T et al (2003) Molecular identification of nicotinic acid receptor. Biochem Biophys Res Commun 303:364-369

367. Tunaru S, Kero J, Schaub A, Wufka C, Blaukat A, Pfeffer K, Offermanns S (2003) PUMA-G and HM74 are receptors for nicotinic acid and mediate its anti-lipolytic effect. Nat Med 9:352-355

368. Wise A, Foord S, Fraser N, Barnes A, Elshourbagy N, Eilert M et al (2003) Molecular identification of high and low affinity receptors for nicotinic acid. J Biol Chem 278:9869-9874

369. Balasse E and Neef M (1973) Influence of nicotinic acid on the rates of turnover and oxidation of plasma glucose in man. Metabolism 22:1193-1204

370. Miettinen T, Taskinen M, Pelkonen R, Nikkilä E (1969) Glucose tolerance and plasma insulin in man during acute and chronic administration of nicotinic acid. Acta Med Scand 186:247-253

371. Wang W, Basinger A, Neese R, Christiansen M, Hellerstein M (2000) Effects of nicotinic acid on fatty acid kinetics, fuel selection, and pathways of glucose production in women. Am J Physiol Endocrinol Metab 279:E50-E59

372. Garg A and Grundy S (1990) Nicotinic acid as therapy for dyslipidemia in non-insulindependent diabetes mellitus. JAMA 264:723-726

373. Santomauro A, Boden G, Silva M, Rocha D, Santos R, Ursich M et al (1999) Overnight lowering of free fatty acids with Acipimox improves insulin resistance and glucose tolerance in obese diabetic and nondiabetic subjects. Diabetes 48:1836-1841

374. Vaag A, Skött P, Damsbo P, Gall M, Richter E, Beck-Nielsen H (1991) Effect of the antilipolytic nicotinic acid analogue acipimox on whole-body and skeletal muscle glucose metabolism in patients with non-insulin-dependent diabetes mellitus. J Clin Invest 88:1282-1290

375. Bajaj M, Suraamornkul S, Kashyap S, Cusi K, Mandarino L, DeFronzo R (2004) Sustained reduction in plasma free fatty acid concentration improves insulin action without altering plasma adipocytokine levels in subjects with strong family history of type 2 diabetes. J Clin Endocrinol Metab 89:4649-4655

376. Daniele G, Eldor R, Merovci A, Clarke G, Xiong J, Tripathy D et al (2014) Chronic reduction of plasma free fatty acid improves mitochondrial function and whole-body insulin sensitivity in obese and type 2 diabetic individuals. Diabetes 63:2812-2820

377. Liang $H$, Tantiwong $P$, Sriwijitkamol A, Shanmugasundaram K, Mohan S, Espinoza S et al (2013) Effect of a sustained reduction in plasma free fatty acid concentration on insulin signalling and inflammation in skeletal muscle from human subjects. J Physiol 591:2897-2909 
378. Davoren P, Kelly W, Gries F, Hubinger A, Whately-Smith C, Alberti K (1998) Longterm effects of a sustained-release preparation of acipimox on dyslipidemia and glucose metabolism in non-insulin-dependent diabetes mellitus. Metabolism 47:250256

379. Vaag A and Beck-Nielsen H (1992) Effects of prolonged Acipimox treatment on glucose and lipid metabolism and on in vivo insulin sensitivity in patients with noninsulin dependent diabetes mellitus. Acta Endocrinol (Copenh) 127:344-350

380. Saloranta C, Groop L, Ekstrand A, Franssila-Kallunki A, Eriksson J, Taskinen M (1993) Different acute and chronic effects of acipimox treatment on glucose and lipid metabolism in patients with type 2 diabetes. Diabet Med 10:950-957 



\section{Chapter 2}

\section{Natriuretic peptides in the control of lipid metabolism and insulin sensitivity}

Kenneth Verboven, Johan WE Jocken, Dominique Hansen, Ellen E Blaak revision submitted 


\section{Abstract}

Natriuretic peptides (NP) have long been known for their cardiovascular function. However, a growing body of evidence emphasizes the role of NP in human substrate and energy metabolism, thereby connecting the heart with several insulin sensitive organs like adipose tissue, skeletal muscle and liver. Obesity may be associated with an impaired regulation of the natriuretic peptide system, also indicated as a natriuretic handicap. Evidence points towards a contribution of this natriuretic handicap to the development of obesity, type 2 diabetes mellitus and cardiometabolic complications, although the causal relationship is not fully understood. Nevertheless, targeting the natriuretic peptide pathway may offer novel future therapeutic potential for improving metabolic health in obesity and type 2 diabetes mellitus. This review will focus on current literature regarding the metabolic roles of NP with emphasis on lipid metabolism and insulin sensitivity. Furthermore, it will be discussed how exercise and lifestyle intervention may modulate the NP-related metabolic effects. 


\section{Introduction}

Obesity is one of the major health problems of the twenty-first century as it is closely associated with the development of chronic metabolic diseases, including cardiovascular disease, insulin resistance (IR) and type 2 diabetes mellitus (T2DM) ${ }^{1-3}$. Different insulin sensitive organs tightly orchestrate energy and substrate metabolism in the human body. Therefore, alterations in these organs may contribute to the development of disturbances in fatty acid (FA) metabolism, ultimately leading to impaired glucose metabolism, IR and cardiometabolic disease. Next to the liver, skeletal muscle, the gastrointestinal tract and the pancreas, the adipose tissue (AT) is an important central organ in the inter-organ crosstalk in human energy and substrate metabolism. The AT is the primary site for long-term energy storage, mainly as triglycerides. However, a chronic excessive energy intake, like in obesity, results in enlargement (hypertrophy) of existing adipocytes. Since adipocytes have a limited expansion capacity, hypertrophy will lead to a reduced buffering capacity and dysfunctional AT may develop 4,5 . This AT dysfunction is further characterized by an altered lipid storage capacity and adipokine release, immune cell infiltration and lowgrade inflammation, plays an important role in the development and/or progression of IR by promoting ectopic lipid storage and systemic low-grade inflammation ${ }^{6-10}$. Physical activity intervention, whether or not combined with diet, may reduce the progression towards T2DM ${ }^{11,12}$, possibly due to modulation of AT, liver and/or skeletal muscle FA metabolism ${ }^{10}$.

Only recently, research proposed natriuretic peptides (NPs) as important endocrine hormones implicated in the regulation of whole-body energy and substrate metabolism ${ }^{13-16}$. Atrial natriuretic peptide (ANP), B-type natriuretic peptide (BNP), both secreted by the heart, and C-type natriuretic peptide (CNP), mainly expressed by vascular endothelial cells and to a lower extent in cardiomyocytes ${ }^{17}$, are the three NP family members that may play a role in human substrate and energy metabolism. Besides their cardiovascular and renal effects (described extensively by Volpe et al. ${ }^{18}$ ), NPs play a role in different metabolic processes including lipid mobilization in human white AT ${ }^{13,15}$, energy dissipation in brown AT, browning of white AT ${ }^{19}$ and fat oxidation in human skeletal muscle ${ }^{20}$, possibly influencing whole-body FA metabolism, glucose 
homeostasis and insulin sensitivity. In addition to their wide range of metabolic effects in human insulin sensitive tissues, plasma NP levels were also reported to be negatively associated with cardiometabolic diseases ${ }^{21-26}$ and have been even suggested to have a predictive value in the development of new onset T2DM ${ }^{25}$. However, a better understanding of the interaction between NPs and tissue energy, FA and glucose metabolism is necessary to obtain a better insight in the role of NPs in the development of IR, T2DM and cardiometabolic complications.

In this review, we will discuss the current knowledge regarding the role of NPs in the control of human lipid metabolism and insulin sensitivity. At first, NP secretion, clearance and signaling is discussed. Subsequently, the role of NPs in the etiology of obesity and T2DM is discussed, as well as their effects on AT, skeletal muscle and liver metabolism. Moreover, the benefits of lifestyle and/or exercise interventions with respect to NPs in the control of insulin sensitivity are considered.

\section{Secretion and processing of NP}

The discovery of the endocrine properties of the heart by deBold in 1981, as shown by a potent natriuretic and diuretic effect of atrial myocardial extracts in rats ${ }^{27}$, led to the reexamination of the function of the earlier discovered atrial myocardium granules ${ }^{28}$. The dual nature of atrial cardiomyocytes (i.e. secretory-contractile function) became obvious and research led to the identification of atrial natriuretic peptide (ANP) ${ }^{29}$ and later the other NPhormone family members B-type natriuretic peptide (BNP), which is found at highest levels in cardiac ventricles, C-type natriuretic peptide (CNP), which is mainly expressed in and produced by endothelial cells ${ }^{17}$. CNP, previously thought to act as a neuropeptide in the central nervous system ${ }^{30,31}$, is mainly viewed as a peptide regulating vascular blood pressure ${ }^{32}$ and bone growth ${ }^{33}$, although a minor role in metabolic regulation has been suggested 34,35 . In mammals, dendroaspis natriuretic peptide (DNP) (of which the synthesis and secretion sites have not been identified) exerts renal actions via its specific receptor ${ }^{36}$ but because it has not been well studied with respect to metabolic effects in humans, DNP will not be further discussed in the current review. 
Currently, ANP and BNP are the main metabolically active NPs described in literature and will therefore be discussed in more detail in the present review. These peptides have a 17-amino acid ring structure in common, formed by an intramolecular disulfide linkage, of which the sequence is highly preserved within the biologically active form of these peptides ${ }^{37}$. Structural differences between NP family members are due to specific amino- and carboxy-terminal extensions 38. At rest, ANP (normal concentration range $5-50 \mathrm{pg} / \mathrm{mL}$ ) is mainly produced by the (right) atrial myocardium as a preprohormone ${ }^{39}$. The intracellular granules of the atrial myocardium contain proANP which is cleaved by corin (type II transmembrane serine protease expressed primarily in the heart) during secretion, resulting in a 28 -amino acid, bioactive aANP peptide ${ }^{40}$, with a very short plasma half-life of about 2-4 min ${ }^{41}$, and inactive fragments ( $\mathrm{N}$-terminal ANP and mid-regional-proANP) which have a longer plasma half-life (about 40$50 \mathrm{~min})^{41,42}$. BNP is mainly produced and secreted by the ventricular myocardium as preproBNP ${ }^{43}$. To become biologically active, preproBNP is cleaved to proBNP and subsequently, like for ANP, a cardiac protease, corin or furin, is responsible for the conversion to the 32-amino acid BNP (range plasma levels $0-65 \mathrm{pg} / \mathrm{mL}$ ) which is secreted in the circulation having a plasma half-life of about $15-25 \min ^{44}$, and the inactive $\mathrm{N}$-terminal fragment proBNP ${ }^{45}$. The latter inactive fragment has a plasma half-life of about 60-120 min and a plasma concentration in the range of $7-220 \mathrm{pmol} / \mathrm{L}$ in healthy individuals ${ }^{41}$. While the structure of BNP varies distinctly among species, ANP is strongly homologous between human and rodent ${ }^{45}$. The post-translational processing, cleavage and degradation sites of NPs were recently reviewed by Volpe et al. ${ }^{18}$.

\section{Determinants of NP secretion}

Mechanical stretch of cardiomyocytes is the most important trigger for NP release in the circulation ${ }^{45}$. Atrial wall stretching causes an increase in ANP gene transcription and increased release of stored granules ${ }^{46}$. Ventricular wall stress, in case of volume or pressure overload, is mainly responsible for BNP transcription and secretion ${ }^{38}$. In addition, hypoxic conditions or myocardial ischemia are potent inducers of ANP secretion due to the presence of hypoxiaresponsive elements in the promotor region of the ANP gene ${ }^{47}$. 
Another potent stimulus for NP secretion is cold exposure. Animals and humans exposed to cold display a greater expression of ANP (mRNA) and BNP (mRNA and protein) in the heart, combined with higher plasma levels, which are suggested to be direct responses of elevated sympathetic nervous system activation in the heart ${ }^{19}$.

Besides mechanical stretch and cold exposure, endocrine regulation of NP secretion is present as well. Sex steroids, thyroid hormones, glucocorticoids, endothelin-1, angiotensin II and inflammatory cytokines (tumor necrosis factora, interleukin-1 and -6) all are able to modulate NP secretion ${ }^{38,45,48}$, the latter stimulating BNP transcription and translation in vitro in murine cardiomyocyte cultures ${ }^{49}$ and in vivo secretion into the plasma in human transplant patients specifically ${ }^{50}$. In this regard, it was shown that the GLP1 receptor agonist liraglutide was able to induce a significant increase in ANP secretion in vivo in T2DM ${ }^{51}$ due to the presence of GLP1 receptors on right atrial cardiomyocytes ${ }^{52}$. ANP and BNP plasma levels also increase with age, possibly due to an agerelated reduction in coronary blood flow reserve and thus increased myocardial ischemia ${ }^{53-55}$. Modulation by sex steroids may result in gender dependent regulation of NP levels ${ }^{55-57}$. The latter is already observed in pubertal versus post-pubertal adolescents, where NP concentrations are lower in post-pubertal boys compared with pubertal boys ${ }^{58}$. Estrogens might have a stimulatory effect on the production and secretion of ANP and BNP by the cardiomyocyte, whereas androgens may have an inhibitory effect ${ }^{56}$. In part, the increased NP levels in women might have clinical implications for gender-related difference in relative risk of developing metabolic and cardiovascular disease. Furthermore, during adolescence NPs levels seem to increase progressively in girls ${ }^{53,54}$, probably the result of an interaction between the increased estrogen concentration and ANP transcription and secretion or via the regulation of the NPs receptor expression 59. Plasma CNP levels alternatively decrease during adolescence until the age of fifty, whereupon they tend to increase. CNP concentrations are higher in men than in women as testosterone and growth hormone are able to induce CNP ${ }^{60}$. Finally, metabolically compromised conditions like obesity, insulin resistance and T2MD may be characterized by altered systemic NP concentrations, which will be discussed more extensively later in this review. 


\section{NP receptors and signaling}

To exert their main biological effects NPs bind to NP receptors, of which three subtypes have been described (reviewed recently by Kuhn) ${ }^{61}$. ANP and BNP bind with a high affinity to a membrane-bound receptor, containing a transmembrane segment, with specific guanylyl cyclase (GC) activity called NP receptor A (NPRA). CNP is mainly bound to NP receptor B (NPRB) ${ }^{62}$, similar in structure and function to NPRA and mainly expressed by chondrocytes, thereby playing a role in long bone growth ${ }^{63}$. Ligand binding to a NPRA homodimer results in the internalization of the bound ligand-receptor complex and the activation of cytosolic GC, the catalytic effector of the receptor, subsequently causing hydrolysis of GTP into cyclic GMP (cGMP). This second messenger is able to activate various biological responses via cGMP-dependent protein kinases, CGMP-gated ion channels or other effector proteins ${ }^{64}$. A large proportion of the ligand-bound receptor undergo lysosomal degradation, in which about $75 \%$ of the internalized ANP is processed in the lysosomes and $25 \%$ is released as intact molecules though a recycling pathway. A small amount of internalized receptors is recycled back to the plasma membrane or released into the cell exterior ${ }^{65}$. The NP receptor C (NPRC) is the third subtype, having an extracellular domain that is partly homologous to those of NPRA receptors and thus has the ability to bind NPs, with the highest affinity to ANP and lowest to BNP ${ }^{66}$. However, NPRC lacks GC activity and instead its main function is to facilitate scavenging of its ligands, internalization of ligand-receptor complexes and recycling of NPRC, together with lysosomal degradation of its ligands ${ }^{67,68}$. Besides via lysosomal clearance, NPs can be degraded intracellularly by endopeptidases including neutral endopeptidase (NEP) ${ }^{69}$, which is also produced in adipocytes ${ }^{70}$. In addition, insulin-degrading enzyme (IDE) enzymatically cleaves NP 45,71 and dipeptidyl peptidase-IV (DPPIV or CD26) cleaves the N-terminal peptide of NPs thereby lowering biological activity ${ }^{72}$. Another route to clear circulating NP is via secretion into body fluids like urine (via glomerular filtration) and bile ${ }^{45}$.

The main effector receptor for ANP and BNP, NPRA, is highly expressed throughout the cardiovascular system (vascular smooth muscle and endothelial cells with only a limited expression in the heart), in kidney and adrenal gland, as well as in different metabolic organs like skeletal muscle, pancreas, liver, brain, 
gut and AT 37,73,74. Expression of the scavenging NPRC, the most widely expressed NPs receptor, is mainly present in the AT, kidneys, lungs, the cardiovascular system and blood monocytes $68,75,76$. Interestingly, NPRA and NPRC display diurnal regulations (in antiphase of one other) in the rodent white AT ${ }^{77}$, not in the heart muscle ${ }^{78}$, which together with the circadian regulated plasma NPs ${ }^{79,80}$, may be a characteristic for energy homeostasis during the day. Furthermore, the local tissue specific and systemic effects of NP are thought to depend on the ratio between the signaling receptor NPRA to the clearance receptor NPRC ${ }^{81,82}$. Collectively, NPs mediate their effects via NP receptors, of which three subtypes have been described. The diverse effects of NPs, systemically as well as the local tissue effects, are determined by NP receptor expression profiles and their ligand-affinity.

\section{Systemic NP deficiency in obesity, insulin resistance and type 2 diabetes mellitus}

Evidence from several epidemiological studies demonstrated an inverse association between systemic NPs levels (both ANP and BNP) and body weight (mostly expressed by body mass index (BMI)) ${ }^{21,23,83-87}$. The inverse relationship between NP concentrations and BMI was found also in the presence of left ventricular hypertrophy ${ }^{88}$. However, these studies lack detailed body composition analyses thereby not being able to differentiate between fat accumulation or fluid retention as a cause of the increased BMI. In contrast, other small cohorts showed that there is no or even a positive relationship between circulating NPs levels and BMI ${ }^{89,90}$. Furthermore, NPs may affect AT distribution ${ }^{91,92}$. Variations in regional and particularly visceral adiposity were strongly related to circulating N-terminal-pro-BNP. The relationship of NPs with subcutaneous adiposity was less strong ${ }^{92}$. This relation could be partly moderated by the hyperinsulinemic state that is frequently observed in visceral adiposity, as high insulin levels have been shown to suppress NPs secretion and activity ${ }^{93,94}$. The Dallas Heart Study recently showed that both BNP and Nterminal-proBNP are inversely related to visceral and liver fat, while being positively associated with lower gluteofemoral body fat, independent of insulin sensitivity ${ }^{95}$. 
Of interest, it is important to take into account obesity comorbidities including the presence of cardiac burden in considering these controversial results ${ }^{96}$ as obesity if often linked to volume overload, leading to left ventricular hypertrophy and left ventricular dysfunction ${ }^{97}$. The decrease in systemic NPs concentrations may be accompanied by higher blood aldosterone concentrations, as a consequence of an impaired NPs-mediated renin-angiotensin-aldosteron system inhibition ${ }^{98,99}$, thereby leading to obesity-related hypertension ${ }^{22}$ or an increased incidence of all-cause mortality ${ }^{86}$. The substantial role of NPs deficiency in the pathogenesis of obesity-related hypertension was recently corroborated in healthy obese men, showing a negative association between serum midregional-proANP and mean 24-hour systolic ambulatory blood pressure ${ }^{100}$. Moreover, research indicated that NPs deficiency might enhance cardiovascular risk ${ }^{18}$. Therefore, achieving normal range NPs levels is preferential with respect to obesity-related hypertension ${ }^{101}$.

As obesity is often associated with IR or T2DM, a link between the NPs system, obesity and T2DM seems plausible. Recent research indicated that NPs deficiency might increase the risk of T2DM onset ${ }^{102}$. Indeed, two recent prospective cohort studies showed evidence supporting this hypothesis 25,26 . Results of the Malmö Diet and Cancer Study showed mid-regional-proANP plasma levels to be inversely associated with new-onset diabetes development (i.e, diabetes incidence) and an impaired glucose metabolism over the 16-year follow-up period of the study, which was also true for $\mathrm{N}$-terminal-proBNP plasma levels, although not statistically significant ${ }^{25}$. In this regard, mid-regionalproANP is believed to be a better predictor of T2DM incidence compared to $\mathrm{N}$ terminal-proBNP 25 , the latter being more sensitive to mild forms of left ventricular dysfunction ${ }^{103}$ which is relatively frequent (even subclinically) in the obese state ${ }^{104}$. Circulating ANP (measured as mid-regional-proANP) within the normal high range $(\sim 212-372 \mathrm{pg} / \mathrm{mL})$ was associated with lower risk of IR during a 16 -year follow-up period in a middle-aged cohort ${ }^{105}$. This association was independent of diabetes risk factors (including waist circumference, plasma levels of triglycerides, HDL-cholesterol, systolic blood pressure, antihypertensive treatment, age and sex) or renal function (cystatin C) ${ }^{25}$. In the Atherosclerosis Risk in Communities Study (a community-based population study), it was shown that having low $\mathrm{N}$-terminal-proBNP levels (lowest quartile, $<31 \mathrm{pg} / \mathrm{mL}$ ) was 
associated with higher risk of incident diabetes over a 12 year follow-up period in subjects without T2DM at baseline. These results were consistent across race, gender and BMI categories ${ }^{26}$, and were independent of age ${ }^{106,107}$. Of interest, statistical adjustment for BMI did not abrogate the association between low NP levels and diabetes onset ${ }^{108}$. These results are in line with the Framingham Heart Study and the Malmö Diet and Cancer Study, which showed that lower Nterminal-proBNP levels were associated with higher incidence of IR in lean as well as in obese subjects in a cross-sectional study ${ }^{23}$. Additionally, prospective cohort data from the Women's Health Study showed that subjects with Nterminal-proBNP levels near the upper limit of the normal range $(>117 \mathrm{pg} / \mathrm{mL})$ have a significantly lower risk of developing diabetes ${ }^{109}$.

Thus, there is consistent evidence that increased NP concentrations are protective against IR 97 and T2DM 109-111. In line, lower blood glucose concentrations were transiently observed upon systemic BNP infusion during intravenous glucose tolerance testing in young, healthy lean men with normal glucose tolerance ${ }^{112}$. Moreover, in a random subset of a general middle-aged population (age $>45$ years) a genetic variant of the ANP gene (single nucleotide polymorphism rs5068) was associated with higher $\mathrm{N}$-terminal-proANP levels and a beneficial cardiometabolic profile (i.e. reduced systemic blood pressure, BMI, waist circumference and a lower risk of metabolic syndrome) compared to the A/A carriers ${ }^{84}$. This ANP gene-polymorphism was accompanied with a lower incidence of T2DM after a 14-year follow-up ${ }^{113}$. Similarly, a genetic polymorphism in the promotor region of the BNP gene ( $T-381 \mathrm{C}$ polymorphism) is associated with higher plasma BNP levels and lower risk of T2DM in several population samples including individuals with normoglycemia, impaired glucose tolerance and T2DM ${ }^{114}$. Together these findings suggest NPs to be protective against IR and development of T2DM.

Altogether, the presence of a NPs deficiency in metabolic disease is generally accepted but the cause remains incompletely understood. Nonetheless, it is imperative to understand the etiology of this anomaly, especially because of the pleiotropic protective cardiovascular and metabolic effects of NPs, as will be described later. Further studies are necessary to establish the clinical relevance of using mid-regional-ANP and/or N-terminal-proBNP as biomarkers for diabetes 
prediction because multiple mechanisms are thought to contribute to the antidiabetic role of NPs ${ }^{15}$.

\section{Underlying mechanism for systemic NP deficiency in obesity}

Several potential explanations for the observed systemic NPs deficiency in human obesity, and more general human metabolic disease, have been proposed, apart from common variants of the human ANP and BNP genes that affect circulating NP concentrations ${ }^{109,111,115}$. One explanation could be that the NP deficiency may be due to an increased NP degradation, which is mainly fulfilled by NPRC-mediated lysosomal breakdown as mentioned before 69,116. Indeed, mRNA expression of NPRC is increased in human AT of obese ${ }^{73,76,81}$ and obese hypertensive patients ${ }^{22}$. In addition, hyperinsulinemia increased NPRC expression in vitro in 3T3-L1 adipocytes ${ }^{81}$, human adipocytes ${ }^{81,117}$ and in human subcutaneous AT of healthy, moderately obese individuals with normal glucose tolerance during hyperinsulinemic-euglycemic and hyperinsulinemichyperglycemic clamps ${ }^{76}$, mainly through the phosphatidylinositol 3-kinase (PI3K) pathway ${ }^{81}$. Together, these data suggest that the AT may be responsible for the increased NP clearance and lowering systemic NP availability in obese insulin resistant conditions ${ }^{76,118}$.

However, results from the Dallas Heart Study showed that the association between BMI and circulating NP levels is explained by the amount of lean mass, and not AT mass, indicating that lean tissue could also be important for plasma NP regulation ${ }^{83}$. Indeed, upregulation of NPRC in human skeletal muscle tissue, next to down-regulation of the NPRA expression in the AT and skeletal muscle of obese and/or obese diabetic humans and mice has been found ${ }^{119,120}$. Thus, besides AT, also skeletal muscle may contribute to the NP deficiency observed in T2DM in the long-term, certainly considering that muscle mass accounts for up to $40 \%$ of total body weight. Therefore, even a moderate increase in skeletal muscle NPRC expression could markedly reduce NPs plasma levels ${ }^{119}$. Moreover, previous work of Sarzani et al. with a genetic NPRC variant shows that a reduced NP clearance (or resulting increased systemic levels) might be associated with a reduced content of upper body fat and a lower risk of developing abdominal obesity ${ }^{91}$. 
Additionally, it has been shown that NPRC mRNA expression is down-regulated in vitro following starvation in human differentiated adipocytes ${ }^{117}$ and in vivo in rat white and brown AT ${ }^{121}$ while the opposite was true under high fat feeding in wild-type mice skeletal muscle, white and brown AT ${ }^{122}$. Like NPRC expression, NEP expression is increased in the plasma (protein) and AT (mRNA) of obese subjects, although there is no direct evidence for an increased NEP activity in human obesity ${ }^{123}$. Together, these findings propose an altered NPs receptor ratio in obese insulin resistant or T2DM individuals mainly due to an increased expression of adipose NPRC and NEP, possibly leading to elevated NP clearance thereby reducing circulating NPs.

On the other hand, a decreased cardiac ANP and BNP release in metabolic disease has also been proposed. Not only circulating NPs levels but also the side products of NPs release, the aminoterminal cleavage products $\mathrm{N}$-terminalproANP and $\mathrm{N}$-terminal-proBNP are reduced in obesity. These proteins are structurally distinct and are biologically inactive compounds, which makes NPRC mediated clearance of these components rather unlikely ${ }^{83,124}$. Of interest, NPs levels in the aortic root and the coronary sinus were observed to be negatively correlated with BMI ${ }^{125}$. These findings suggest that besides the above indicated impaired clearance, a reduced cardiac NPs release might potentially contribute to the systemic NPs deficiency in metabolic diseases as well. This hypothesis was further acknowledged by the notion that cardiac ANP and BNP mRNA expressions were reduced in obese Zucker fatty rats and $d b / d b$ mice ${ }^{126,127}$. Putative impairments in cardiac NPs secretion in human metabolically compromised conditions needs to be further explored.

\section{NP and its role in inter-organ crosstalk}

NPs are long known for their cardiovascular and renal actions ${ }^{18}$ resulting in the use of NPs (or their fragments) as cardiovascular biomarkers in the clinic ${ }^{128}$. Over the last two decades, pioneering studies by the group of Lafontan et al. suggested a protective role for the heart in metabolic diseases ${ }^{129}$. The interorgan crosstalk effectuated by ANP and BNP secretion by the heart integrates effects on AT function, skeletal muscle, liver, gut, central nervous system and pancreas, as indicated in Figure 1. 


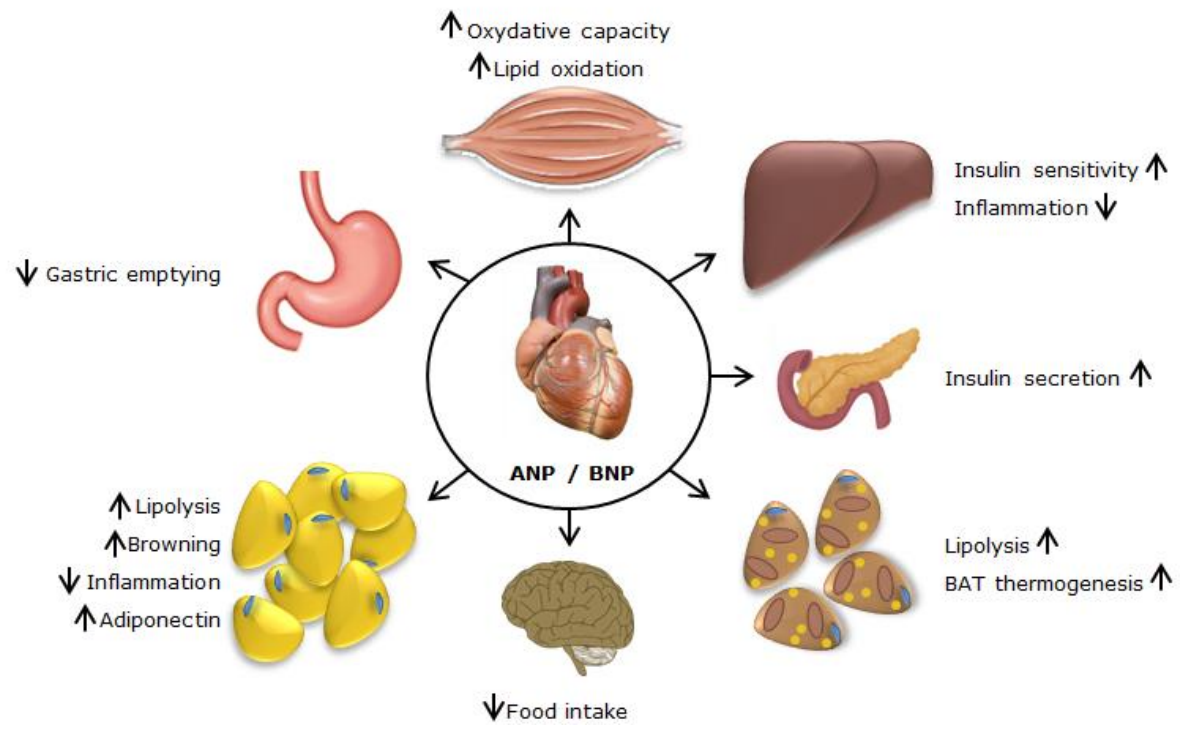

Figure 1 - Overview of the multiple metabolic actions of natriuretic peptides in the control of lipid metabolism and insulin sensitivity.

\section{NP and adipose tissue function}

As the human white AT is one of the main sites of NP receptor expression, investigating the effects of NPs on AT function seems obvious, in particular in the obese state where AT mass is expanded. Next, the effects on AT lipolysis, brown AT metabolism and the role of NP in AT lipid metabolism in obesity and T2DM will be discussed in more detail.

\section{NP and adipose tissue lipolysis}

The potency of inducing a lipolytic effect by NPs was first described in isolated human adipocytes, with ANP being the most potent activator of lipolysis as compared to BNP and CNP, the latter only showing a minor maximal lipolytic response ( $17 \%$ as compared to maximal stimulation with isoproterenol) ${ }^{129}$. In addition, these lipolytic properties seem to be primate-specific, which may be due to the differential NPRC clearance receptor expression profiles in other mammalian adipocytes, especially in rodents, making ANP-mediated lipolysis less likely due to increased NPs clearance and degradation ${ }^{130}$. The signaling 
pathway relies on cGMP-dependent activation of protein kinase G (PKG), thereby promoting phosphorylation of perilipin-1 (PLIN-1) and hormone sensitive lipase (HSL) to trigger triglyceride hydrolysis $129,131,132$, in which adipose triglyceride lipase (ATGL) might be involved as well ${ }^{117}$, the latter probably via a different signaling pathway (i.e. AMP-activated kinase) ${ }^{133}$ as compared with HSL activation (i.e. PKA and PKG) ${ }^{134}$. NPs-induced lipolysis is completely independent from the catecholamine-induced (CAMP/PKA mediated) lipolysis, as they rely on different pathways ${ }^{135,136}$. However, an additive lipolytic effect occurs when human adipocytes are stimulated with ANP and a beta-adrenergic agonist (e.g. isoproterenol) simultaneously ${ }^{137}$. Infusion studies of human ANP (hANP), either intravenous or in situ through microdialysis in the subcutaneous AT, showed promotion of lipid mobilization in healthy subjects, also in the presence of local beta-adrenergic blockade ${ }^{135}$. Infusing intravenous hANP (doses from 6.25-25 $\mathrm{ng}^{*} \mathrm{~kg}^{-1} \mathrm{~min}^{-1}$ ), corresponding to the physiological range observed during moderate exercise, stimulates whole-body lipid mobilization and oxidation (in a dose dependent way), even in the postprandial state 132,136,138. Furthermore, exercise-induced increases in systemic ANP concentrations (which may vary depending on the exercise/subjects' characteristics) lead to an increase in lipid mobilization, at least in lean healthy subjects ${ }^{139}$. In human obesity, lipolytic catecholamine-resistance is most commonly observed in the subcutaneous AT in the obese insulin resistant state ${ }^{140-142}$. In line, regarding ANP-mediated lipolysis, an impaired ANP-mediated lipolysis in vitro and in situ in human subcutaneous AT of obese women and overweight men compared to non-obese counterparts has been reported ${ }^{120}$. ANP-mediated lipid mobilization was reported to be comparable in subcutaneous adipocytes compared to visceral adipocytes of obese women ${ }^{143}$. The blunted ANP-mediated lipolysis in the subcutaneous AT of obese insulin resistant individuals may be in part due to an up-regulation of NPRC and a down-regulation of NPRA mRNA and protein in human subcutaneous adipocytes ${ }^{120}$.

An interaction between the NPs' system and the anti-lipolytic hormone insulin was first suggested by Endre et al. ${ }^{144}$, who showed that hyperinsulinemic euglycemic clamping caused a decrease in serum ANP in normotensive and hypertensive men. Insulin inhibits the catecholamine-induced lipolysis via activation of phosphodiesterase 3B (PDE3B), but does not have a direct anti- 
lipolytic effect on the CGMP-PKG dependent lipolytic pathway of ANP ${ }^{147}$. However, insulin might attenuate ANP-mediated lipolysis by inducing NPRC expression, as described earlier in this review. Of interest, the presence of lowglucose conditions together with insulin stimulation abolished NPRC expression to basal levels, indicating the existence of a "nutritional signaling" in NPRC regulation ${ }^{117}$. The relative ratio of NPRA to NPRC mRNA levels in subcutaneous AT was decreased depending on glucometabolic status as patients with T2DM had the lowest ratio compared to subjects with normal glucose tolerance or impaired glucose metabolism ${ }^{146}$. In parallel with improvements in insulin sensitivity, 12 weeks of treatment with pioglitazone, a thiazolidinedione that affects insulin sensitivity ${ }^{147}$, increased the relative NPRA to NPRC MRNA ratio with the greatest effect on NPRC mRNA levels in subcutaneous AT in patients with T2DM ${ }^{146}$. Acute increases in systemic blood glucose decreased circulating $\mathrm{N}$-terminal-proANP in lean, overweight and obese subjects, a mechanism mediated through glucose-induced miR-425 expression ${ }^{148}$, a negative regulator of NPRA ${ }^{149}$. Insulin thus seems to be a key hormone in the connection between glucose/lipid metabolism and NPs' metabolic activities.

Together, one can suggest that improving NP-mediated lipid mobilization in the obese insulin resistant and/or T2DM state might be a potential novel strategy to improve lipid metabolism in metabolic disease.

\section{NP and adipokines}

Another way to link NPs to AT function is the ability of NPs to alter expression and secretion of adiponectin, an adipokine with insulin sensitizing properties, both in vitro ${ }^{150}$ and in vivo ${ }^{151}$ in healthy subjects. Moreover, adiponectin is positively associated with NPs $95,106,152,153$. Other insulin desensitizing mediators frequently linked to NPs include TNF-a or interleukin-6. The secretion of these pro-inflammatory factors was reduced by treating human AT pieces with physiological ANP concentrations in vitro, possibly through a direct effect on both adipocytes and macrophages ${ }^{154}$. In this regard, reducing pro-inflammatory cytokines and increasing adiponectin secretion from AT could indirectly ameliorate insulin sensitivity by NPs. 


\section{NP and brown adipose tissue metabolism}

Next to white AT, the presence and the importance of human brown AT (BAT) for human metabolic diseases receives increasing attention ${ }^{155-158}$, although the quantitative importance of BAT in human energy and substrate metabolism remains uncertain. BAT is a thermogenic tissue having the ability to dissipate energy in the form of heat, thereby maintaining body temperature. Substrates including glucose and free fatty acids, delivered by white AT lipolysis, are necessary for heat dissipation, a process that is mediated by mitochondrial inner membrane uncoupling protein 1 (UCP-1) ${ }^{159}$. In addition, UCP-1 may have a regulatory function in whole-body energy homeostasis ${ }^{160}$. However, most of these data are derived from rodent studies and because adult human BAT may have a differential gene expression profile as either rodent BAT or beige fat ${ }^{161}$, it physiological function in humans still needs to be determined in more detail. Support for a role of NPs in non-shivering thermogenesis was recently provided by showing that cold exposure acutely increases cardiac BNP secretion in mice, resulting in an increased NPRA/NPRC ratio in white AT ${ }^{19}$. Mechanistic experiments indeed showed that ANP (and BNP) might activate mitochondrial biogenesis and uncoupling in human and mouse white adipocytes, in an AMPK dependent manner ${ }^{19}$. Chronic BNP treatment of $d b /+$ and $d b / d b$ mice further confirmed these findings, showing increased UCP-1 expression and browning of the white fat pads ${ }^{126}$. ANP treatment also enhanced mitochondrial function in human adipocytes ${ }^{162}$. Taken together, studies have shown that the NP system is able to induce a thermogenic process in the AT and to induce brown AT activation. However, this knowledge is only limited to in vitro experiments. Since cold exposure is able to increase both NPs secretion and brown AT activation, the role of NPs in white AT "browning" is of particular interest in the human in vivo situation, making this connection a potential target for treating metabolic disease. Until today, the role of NPs in human brown AT remains elusive.

\section{NP and skeletal muscle metabolism}

The mobilization of free fatty acids from AT depots by NPs provides substrates for energy production by oxidative tissues 132,135 . However, not only AT lipid mobilization has been shown to be susceptible to NPs but also enhancement of AT and muscle lipid oxidation. Birkenfeld et al. observed an acute increase in 
whole-body lipid oxidation (predominantly resulting from increased muscle lipid oxidation) following intravenous ANP infusion. Additionally ANP infusion leads to higher energy expenditure in the postprandial state ${ }^{138}$. The oxidative effect of ANP, as well as BNP, was later confirmed in vitro in human muscle cells and in vivo in mice (in vitro and in vivo) showing increased skeletal muscle mitochondrial biogenesis, respiration and lipid oxidation upon chronic overexpression of BNP or cGMP-dependent protein kinase in transgenic mice ${ }^{122}$. This enhanced oxidative metabolic state was protective for high fat diet induced obesity and glucose intolerance in mice. A physiological role of NPs in the regulation of skeletal muscle oxidative capacity in human primary myotubes was established by showing that ANP, BNP and CGMP analogs induce peroxisome proliferator-activated receptor gamma coactivator 1-alpha (PGC-1a) expression, mitochondrial oxidation and lipid (palmitate) oxidation in vitro ${ }^{20}$. In addition, PGC-1a expression was associated with NPRA expression in skeletal muscle of healthy human subjects ${ }^{20}$. This proposes that NPs affect mitochondrial respiration and lipid oxidation in skeletal muscle through a cGMP dependent pathway, which was shown to be mediated by the induction of transcription and protein expression of PGC-1a and several OXPHOS complexes (complex I and complex IV), accompanied by an unchanged peroxisome proliferator activated receptor (PPAR) $\delta$ expression and mitochondrial DNA content ${ }^{20}$.

Moreover, in skeletal muscle of obese and glucose intolerant humans and mice an altered NPRA/NPRC protein ratio was recently reported to be accompanied with a diminished phosphorylation and activation of p38 MAPK, a downstream effector of the NPs receptor signaling pathway. However, chronic treatment of mice with obesity-induced glucose intolerance and T2DM with BNP showed a reduced total diacylglycerol content in skeletal muscle, which was accompanied with higher oxidative capacity and PGC-1a gene expression ${ }^{119}$. This observation was further confirmed in human primary myotubes, showing that increased NP mediated lipid oxidation was accompanied by reduced de novo ceramide production ${ }^{119}$. These studies indicate the importance of NPs signaling in skeletal muscle lipid oxidative capacity, which is imperative for long-term maintenance of insulin sensitivity in obesity and T2D. 
Yet, recent data indicate a conceivable interaction between the NPs system and an exercise induced myokine called musclin ${ }^{163}$. This skeletal muscle protein is homologous to members of the NPs family ${ }^{163}$ and their interaction results in the amelioration of the NPs' effectiveness ${ }^{164}$. Its physiologically relevant interaction was shown in vitro and in vivo and indicates the NPRA-mediated increase in skeletal muscle mitochondrial biogenesis to be potentiated by a musclin-NPRC interaction during exercise in mice ${ }^{164}$. Musclin is significantly upregulated in skeletal muscle of obese IR mice ${ }^{163}$ and its gene expression is known to be increased upon palmitate treatment in $\mathrm{C} 2 \mathrm{C} 12$ myotubes ${ }^{165}$ and high fat diet in rats ${ }^{166}$. Therefore, dysregulation of musclin might play a role in IR of skeletal muscle and thus needs further investigation to elucidate the molecular mechanisms by which expression of muscle can be changed in human metabolic conditions. Furthermore, as musclin was proposed to exert its effects on glucose uptake in skeletal muscle via PPAR- ${ }^{167}$, this also suggests a possible role for musclin in AT metabolism which needs to be explored in humans in the future.

\section{NP and liver function}

Hepatic IR is an additional hallmark frequently observed in the metabolic syndrome and develops in case of an imbalance between fatty acid supply and utilization of fatty acids by hepatocytes and myocytes, leading to ectopic lipid accumulation and whole-body IR ${ }^{168}$. In obesity the presence of non-alcoholic fatty liver (NAFL) is frequently observed ${ }^{169}$, which may further lead to nonalcoholic steatohepatitis, liver cirrhosis or even liver carcinoma ${ }^{170}$. Recent studies show inverse relationships between NPs, in particular N-terminalproBNP, and liver fat content in individuals without diabetes or self-reported liver disease ${ }^{171}$, as well as between NPs and liver function as indicated by aminotransferases enzymes in individuals without cardiovascular disease ${ }^{172}$. Additionally, NPs could ameliorate hepatic function as the presence of NPs receptors was shown in the human liver ${ }^{173}$. More precisely, these receptors were found on Kupffer-cells, resulting in a hepatoprotective effect of ANP by reducing Kupffer-cell-derived oxidant stress ${ }^{174}$ and inhibiting LPS-induced release of pro-inflammatory TNF- $\alpha$ via a cGMP-mediated signaling ${ }^{175}$. ANP or its analogs inhibited hepatic glycolysis and stimulated gluconeogenesis and cGMP production in perfused livers of fed rats ${ }^{176}$. Besides, ANP also induces hepatic 
lipid oxidation in healthy lean individuals, thereby reducing lipid spill-over and ectopic lipid deposition ${ }^{138}$. Consequently, lower liver TAG content was observed in BNP- or CGKI-transgenic mice on a high fat diet ${ }^{122}$. These findings were later confirmed in a cGKI knock out model, indicated by the presence of IL- 6 mediated liver inflammation, fasting hyperglycemia and reduced insulin signaling 177. These data together suggest a direct role of NPs in liver lipid catabolism next to indirect effects via AT mass reduction ${ }^{122}$.

\section{NP and control of insulin sensitivity}

A role of NPs in the regulation of insulin sensitivity is plausible since several studies indicated an inverse association between NP deficiency and IR in human cohorts 21,24,87,108. Moreover, the broad range of metabolic effects in insulin sensitive tissues makes these peptides putative targets for lifestyle and exercise interventions in metabolic diseases like obesity and T2DM. By enhancing adipocyte lipid mobilization, AT function and skeletal muscle oxidative capacity, NP might contribute to a reduction in ectopic lipid accumulation and thus an improved insulin sensitivity. However, unraveling the mechanistic link between NPs signaling and insulin sensitivity remains incompletely understood.

\section{NP and insulin sensitivity}

A role of NPs and their signaling pathway(s) in the control of insulin sensitivity was corroborated by BNP- and cGK-I-transgenic animals, which were protected against the development of diet-induced IR and glucose intolerance ${ }^{122}$. In line, insulin sensitivity and glucose tolerance improved with chronic BNP infusion in obese diabetic $\mathrm{db} / \mathrm{db}$ mice ${ }^{119,126}$, which was accompanied by a reduced lipid content in liver and skeletal muscle ${ }^{119,122,126}$. On the contrary, genetic knockdown of the NPs signaling cascade impaired fasting glycemia in mice, possibly reflecting an attenuated insulin-mediated regulation of hepatic gluconeogenesis 177. Mechanisms responsible for the improvements in the NP-induced metabolic effects may relate to NP secretion as well as NPs receptor expression and postreceptor signaling. Indeed, in humans, whole-body insulin sensitivity was recently shown to strongly correlate with NPRA in subcutaneous AT ${ }^{146}$ and skeletal muscle ${ }^{119}$. Next to the observed downregulated NPRA (both at mRNA and protein) in obese insulin resistant individuals ${ }^{120,146}$, AT ${ }^{146}$ and skeletal 
muscle ${ }^{119}$ NPRC expression was increased and was negatively associated with whole-body insulin sensitivity. Furthermore, caloric restriction-induced weight loss resulted in an increased NPRA/NPRC ratio in the skeletal muscle tissue of obese subjects and was related to improvements in insulin sensitivity ${ }^{119}$. Additionally, NP degradation by NEP ${ }^{69}$ may contribute to the development of IR (as was shown in obese Zucker rats) ${ }^{178,179}$. Indeed NEP expression in plasma and adipocytes was positively associated with obesity and cardiometabolic risk in the presence of IR ${ }^{123}$.

\section{NP and insulin secretion}

In addition to changes in insulin sensitivity, NPs may also affect pancreatic insulin secretion. Increased insulin levels were observed during ANP infusion in healthy subjects ${ }^{138,180}$, while others showed no alterations during physiological infusion ${ }^{181}$. This effect of NPs on insulin concentration could be (partly) mediated by an increased secretion since NPRA receptors were shown to be present on pancreatic $a$ and $\beta$ cells ${ }^{182}$. Furthermore, $\beta$ cell insulin content, fasting glycemia as well as islet size and $\beta$ cell mass were shown to be attenuated in the NPRA knock out state ${ }^{182}$, the latter being confirmed in isolated rat pancreatic islands ${ }^{183}$.

\section{Impact of exercise/lifestyle intervention on NP-related metabolic effects}

The use of exercise as a non-pharmacological strategy to combat NP deficiency is appropriate as several studies have shown that exercise increases ANP levels, at least in healthy subjects ${ }^{139,184-186}$. In particular, ANP secretion is enhanced by increasing venous return and cardiac filling pressure (i.e. cardiac output) ${ }^{187-189}$, while only slightly increasing ${ }^{187}$ or even not altering circulatory BNP levels ${ }^{188}$ in healthy volunteers, possibly indicating opposing regulation in cardiac atria and ventricles. One study reported plasma BNP levels to be positively associated with physical activity levels, although this remains controversial ${ }^{190,191}$. Exercise in elderly, healthy subjects caused significantly increased proANP and Nterminal-proBNP levels ${ }^{192}$. Of interest, data about exercise effects on NPs in metabolic conditions are scarce. Tanaka et al. showed that NP secretion (ANP and BNP) is more sensitive to sympathetic activity in normotensive subjects 
compared to hypertensive patients, where increased NPs levels may represent cardiac stress ${ }^{193}$. In overweight and obese patients mid-regional-proANP concentrations increased upon incremental exhaustive exercise both before and after diet intervention, with no difference in exercise response between these conditions ${ }^{194}$. Moreover, NPs were also evaluated in relation to resistance training ${ }^{195,196}$. Resistance training induces a significant increase in $\mathrm{N}$-terminalproBNP, which might be partly due to myocardial damage ${ }^{195}$. However, $\mathrm{N}$ terminal-proBNP concentrations did not change in elderly following resistance training ${ }^{196}$. The latter two studies indicate that the effects of strength training on NPs concentrations are still controversial and thus need more investigation, especially in metabolically compromised conditions.

With respect to CNP, exercise preconditioning (that is, an enhancement of the tolerance of an ischemic heart to subsequent ischemic insult by a single bout of interval exercise) promotes the secretion of CNP in rodents, thereby elevating CNP levels in the myocardium and protecting against high-intensity exerciseinduced myocardial injury ${ }^{197}$. Indeed, exercise training (more specifically walking or walking plus resistance exercise training) increased plasma CNP levels, which may be one of the mechanisms through which exercise intervention may reverse endothelial-dependent dysfunction in middle-aged individuals with impaired glucose tolerance ${ }^{198}$. This supports the use of (combined) exercise training to prevent T2DM.

\section{NP and its role in intervention-induced adipose tissue and muscle phenotype changes}

However, mechanisms responsible for the exercise-/exercise training-induced enhancement of the NP deficiency in metabolic disease might include multiple paths. In this respect, AT expression levels of functional NPRA do increase with regular physical activity ${ }^{199}$, an effect that was absent after caloric restriction ${ }^{194}$. In line, long-term aerobic exercise training (partly) recovered the reduced ANP responsiveness in situ in white AT of overweight subjects ${ }^{186}$ and in obese women with polycystic ovary syndrome in vitro and in vivo, independently of AT mass loss ${ }^{200}$. Additionally, ANP-induced increase in AT blood flow, a functional AT characteristic, which has been shown to be impaired in young obese men ${ }^{135}$, 
also improved following long-term aerobic exercise training ${ }^{186}$. As an alternative, water-based exercise, which is known to be beneficial compared to land-based exercise regarding injury risk and joint stress in obesity, has been shown to be not advantageous over moderate intensity land-based exercise with respect to lipid mobilization or fat oxidation ${ }^{201}$. However, exercise in water did show higher circulatory ANP levels in rats ${ }^{202}$ and in humans despite no change in lipid oxidation 201. Moreover, as exercise training is supposed to enhance mitochondrial function at the level of the skeletal muscle ${ }^{203}$, this improvement could be partly due to an increased NPRA expression and signaling as indicated by increased expression of PGC1-a and several oxidative phosphorylation genes following an 8-week aerobic training program in obese subjects ${ }^{20}$. Of interest, the anti-diabetic drug pioglitazone improves insulin sensitivity, which was accompanied by an increased NPRA/NPRC ratio in subcutaneous AT ${ }^{146}$. Liraglutide-induced weight loss in obese subjects with T2DM was correlated with change in NPs levels, although the mechanism responsible remained elusive ${ }^{51}$. Together, these data indicate that the NPs signaling pathway may be a suitable target to improve insulin sensitivity in exercise-based lifestyle interventions.

Diet-induced 130 and gastric bypass-mediated weight loss ${ }^{120}$ confirmed the above indicated reversibility of the reduced maximal ANP responsiveness in the subcutaneous AT of obese women, postulating that the observed impairments in NPs-mediated metabolic effects are secondary to the obese state. With respect to caloric restriction, Sarzani et al. showed that fasting restored NPs signaling by reducing NPRC expression in the AT ${ }^{121}$ which was later confirmed by others ${ }^{122}$. Besides, weight loss studies, either involving caloric restriction or gastric bypass surgery, indicated increased systemic NPs levels (i.e. NT-proBNP) 204-209, which was not confirmed in all studies $194,210,211$. Comparing these interventions, it was recently shown that gastric bypass surgery resulted in a greater systemic increase in NT-proBNP as compared to lifestyle intervention in morbidly obese patients 212 , indicating that the amount of weight loss is associated with the increase in systemic NPs concentrations. Non-pharmacological interventions attenuate NPs levels by altering their signaling pathways and consequently their metabolic effects, which are proposed to result in an improve insulin sensitivity. However, as many studies also observe changes in the inactive fragments (NTproANP and NT-proBNP), which are not cleared by NPRC, these findings suggest 
not only changes in signaling but also adjustments in cardiac production and release following weight loss. In this regard, improvements in other comorbidities which could affect the NPs system following this type of interventions should be taken into account as well.

Despite, the use of exercise training, weight loss interventions or a combination between both, and their interaction with NPs related improvements in insulin sensitivity need further investigation. However, the present clinical studies do not indicate a causal relationship but they do indicate the presence of a strong association between the NPs system and insulin sensitivity management, which needs further focus in upcoming human non-pharmacological intervention studies. 


\section{Conclusions}

The potential role of NPs as an important metabolic target affecting insulin sensitivity in metabolically comprised conditions has been put forward over the last decade. Preclinical and clinical research indicated the presence of a NP deficiency in obesity and T2DM, which is a well-accepted anomaly that could result from inadequate cardiac NPs production and secretion, as well as an increased degree of peripheral degradation. Of interest, several impairments in NPs receptor and post-receptor signaling have been observed in peripheral tissues like the AT or the skeletal muscle tissue of patients with metabolic disease, including overweight, obesity, IR and type 2 diabetes. Despite several animal and human data suggest a causal link between these NPs (signaling) deficiencies and the development of T2DM, the exact molecular mechanism remains incompletely understood. Unraveling the molecular background of these anomalies could therefore further highlight the emerging role of NPs in metabolic diseases. From a clinical point of view, it remains important to investigate therapeutic options to restore this NP deficiency. Next to implementing pharmacological intervention strategies, lifestyle interventions (including physical activity and diet) are of interest in the treatment of metabolic disease. Indeed, previous studies indicated diet-induced weight loss and physical exercise training to sensitize NPs signaling in AT and skeletal muscle tissue, two main metabolic organs with a role in obesity-related IR and T2DM. However, selecting the appropriate intervention with respect to reducing cardiovascular and metabolic risk factors is of special interest to further improve cardiometabolic health and reduce the development and progression of obesity-related risk factors. Therefore, long-term human intervention studies are needed to clarify the role of NPs on control of body weight and insulin sensitivity, including investigation of the underlying molecular machinery. 


\section{References}

1. Shulman G (2014) Ectopic fat in insulin resistance, dyslipidemia and cardiometabolic disease. N Engl J Med 371:1131-1141

2. Grundy S (2004) Obesity, metabolic syndrome, and cardiovascular disease. J Clin Endocrinol Metab 89:2595-2600

3. Kahn S, Hull R, Utzschneider K (2006) Mechanisms linking obesity to insulin resistance and type 2 diabetes. Nature 444:840-846

4. Krotkiewski M, Björntorp $P$, Sjöström L, Smith U (1983) Impact of obesity on metabolism in men and women. Importance of regional adipose tissue distribution. J Clin Invest 72:1150-1162

5. Srdic B, Stokic E, Korac A, Ukropina M, Velickovic K, Breberina M (2010) Morphological characteristics of abdominal adipose tissue in normal-weight and obese women of different metabolic profiles. Exp Clin Endocrinol Diabetes 118:713718

6. Goossens G (2008) The role of adipose tissue dysfunction in the pathogenesis of obesity-related insulin resistance. Physiol Behav 94:206-218

7. Unger R, Clark G, Scherer P, Orci L (2010) Lipid homeostasis, lipotoxicity and the metabolic syndrome. Biochim Biophys Acta 1801:209-214

8. Virtue S, Vidal-Puig A (2010) Adipose tissue expandability, lipotoxicity and the Metabolic Syndrome--an allostatic perspective. Biochim Biophys Acta 1801:338-349

9. Snel M, Jonker J, Schoones J, Lamb H, de Roos A, Pijl H et al (2012) Ectopic fat and insulin resistance: pathophysiology and effect of diet and lifestyle interventions. Int J Endocrinol 2012:983814

10. Stinkens R, Goossens G, Jocken J, Blaak E (2015) Targeting fatty acid metabolism to improve glucose metabolism. Obes Rev 16:715-57

11. Roumen C, Blaak E, Corpeleijn E (2009) Lifestyle intervention for prevention of diabetes: determinants of success for future implementation. Nutr Rev 67:132-146

12. Tuomilehto J (2009) Nonpharmacologic therapy and exercise in the prevention of type 2 diabetes. Diabetes Care 32 Suppl 2:S189-S193

13. Lafontan M, Moro C, Berlan M, Crampes F, Sengenes C, Galitzky J (2008) Control of lipolysis by natriuretic peptides and cyclic GMP. Trends Endocrinol Metab 19:130-137

14. Moro C, Smith S (2009) Natriuretic Peptides: New Players in Energy Homeostasis. Diabetes 58:2726-2728

15. Moro C, Lafontan M (2013) Natriuretic peptides and cGMP signaling control of energy homeostasis. Am J Physiol Heart Circ Physiol 304:H358-H368

16. Coué M, Moro C (2016) Natriuretic peptide control of energy balance and glucose homeostasis. Biochimie 124:84-91

17. Del Ry S, Cabiati M, Vozzi F, Battolla B, Caselli C, Forini F et al (2011) Expression of C-type natriuretic peptide and its receptor NPR-B in cardiomyocytes. Peptides 32:1713-1718

18. Volpe M (2014) Natriuretic peptides and cardio-renal disease. In J Cardiol 176:630639

19. Bordicchia M, Liu D, Amri E, Ailhaud G, Dessi-Fulgheri P, Zhang C et al (2012) Cardiac natriuretic peptides act via p38 MAPK to induce the brown fat thermogenic program in mouse and human adipocytes. J Clin Invest 122:1022-1036

20. Engeli S, Birkenfeld A, Badin P, Bourlier V, Louche K, Viguerie N et al (2012) Natriuretic peptides enhance the oxidative capacity of human skeletal muscle. J Clin Invest 122: 4675-4679 
21. Wang T, Larson M, Levy D, Benjamin E, Leip E, Wilson P et al (2004) Impact of obesity on plasma natriuretic peptide levels. Circulation 109:594-600

22. Dessi-Fulgheri P, Sarzani R, Tamburrini P, Moraca A, Espinosa E, Cola G et al (1997) Plasma atrial natriuretic peptide and natriuretic peptide receptor gene expression in adipose tissue of normotensive and hypertensive obese patients. J Hypertension 15:1695-1699

23. Khan A, Cheng S, Magnusson M, Larson M, Newton-Cheh C, McCabe E et al (2011) Cardiac natriuretic peptides, obesity, and insulin resistance: evidence from two community-based studies. J Clin Endocrinol Metab 96:3242-3249.

24. Walford G, Ma Y, Christophi C, Goldberg R, Jarolim P, Horton E et al (2014) Circulating natriuretic peptide concentrations reflect changes in insulin sensitivity over time in the Diabetes Prevention Program. Diabetologia 57:935-939

25. Magnusson M, Jujic A, Hedblad B, Engstrom G, Persson M, Struck J et al (2012) Low plasma level of atrial natriuretic peptide predicts development of diabetes: the prospective Malmo Diet and Cancer study. J Clin Endocrinol Metab 97:638-45

26. Lazo M, Young J, Brancati F, Coresh J, Whelton S, Ndumele C et al (2013) NH2terminal pro-brain natriuretic peptide and risk of diabetes. Diabetes 62:3189-3193

27. De Bold A, Borenstein H, Veress A, Sonnenberg H (1981) A rapid and potent natriuretic response to intravenous injection of atrial myocardial extract in rats. Life Sci 28:89-94

28. Kisch B (1956) Electron microscopy of the atrium of the heart. I Guinea pig. Exp Med Surg 14:99-112

29. Kangawa K, Fukuda A, Minamino N, Matsuo H (1984) Purification and complete amino acid sequence of beta-rat atrial natriuretic polypeptide (beta-rANP) of 5,000 daltons. Biochem Biophys Res Commun 119:933-940

30. Komatsu Y, Nakao K, Suga S, Ogawa Y, Mukoyama M, Arai H et al (1991) C-type natriuretic peptide (CNP) in rats and humans. Endocrinology 129:1104-1106

31. Sudoh T, Minamino N, Kangawa K, Matsuo H (1990) C-type natriuretic peptide (CNP): a new member of natriuretic peptide family identified in porcine brain. Biochem Biophys Res Commun 168:863-870

32. Moyes A, Khambata R, Villar I, Bubb K, Baliga R, Lumsden N et al (2014) Endothelial C-type natriuretic peptide maintains vascular homeostasis. J Clin Invest 124:40394051

33. Kake T, Kitamura H, Adachi Y, Yoshioka T, Watanabe H, Fujii T et al (2009) Chronically elevated plasma C-type natriuretic peptide level stimulates skeletal growth in transgenic mice. Am J Physiol Endocrinol Metab 297:E1339-E1348

34. Inuzuka M, Tamura N, Yamada N, Katsuura G, Oyamada N, Taura D et al (2010) Ctype natriuretic peptide as a new regulator of food intake and energy expenditure. Endocrinology 151:3633-3642

35. Yamada-Goto N, Katsuura G, Ebihara K, Inuzuka M, Ochi Y, Yamashita Y et al (2013) Intracerebroventricular administration of C-type natriuretic peptide suppresses food intake via activation of the melanocortin system in mice. Diabetes 62:1500-1504

36. Park B, Kim S, Kim S, Noh H, Cho C, Kim S (2015) Characteristics of dendroaspis natriuretic peptide and its receptor in streptozotocin-induced diabetic rats. Mol Med Rep 12:2969-2976

37. Gardner D, Chen S, Glenn D, Grigsby C (2007) Molecular biology of the natriuretic peptide system: implications for physiology and hypertension. Hypertension 49:419426

38. Nishikimi T, Kuwahara K, Nakao K (2011) Current biochemistry, molecular biology, and clinicalrelevance of natriuretic peptides. J Cardiol 57:131-140 
39. Nakao K, Ogawa Y, Suga S, Imura H (1992) Molecular biology and biochemistry of the natriuretic peptide system. I: Natriuretic peptides. J Hypertension 10:907-912

40. Yan W, Wu f, Morser J, Wu Q (2000) Corin, a transmembrane cardiac serine protease, acts as a pro-atrial natriuretic peptide-converting enzyme. Proc Natl Acad Sci USA 97:8525-8529

41. Clerico A. Natriuretic peptides - The hormones of the heart. Clerico A, Emdin M, Eds. Springer Science+ Business Media, 2006

42. Morgenthaler N, Struck J, Thomas B, Bergmann A (2004) Immunoluminometric assay for the midregion of pro-atrial natriuretic peptide in human plasma. Clin Chem 50:234-236

43. Nannipieri M, Seghieri G, Catalano C, Prontera T, Baldi S, Ferrannini E (2002) Defective regulation and action of atrial natriuretic peptide in type 2 diabetes. Horm Metab Res 34:265-270

44. Nakayama K (1997) Furin: a mammalian subtilisin/Kex2p-like endoprotease involved in processing of a wide variety of precursor proteins. Biochem J 327:625-635

45. Potter L (2011) Natriuretic peptide metabolism, clearance and degradation. FEBS J 278:1808-1817

46. McGrath M, de Bold M, de Bold A (2005) The endocrine function of the heart. Trends Endocrinol Metab 16:469-477

47. Chun S, Hyun J, Kwak Y, Kim I, Kim C, Choi E et al (2003) Hypoxic activation of the atrial natriuretic peptide gene promoter through direct and indirect actions of hypoxia-inducible factor-1. Biochem J 370:149-157

48. Ogawa Y, Itoh H, Nakao K (1995) Molecular biology and biochemistry of natriuretic peptide family. Clin Exp Pharmacol Physiol 22:49-53

49. Ogawa T, de Bold A (2012) Brain natriuretic peptide production and secretion in inflammation. J Transplant 2012:962347

50. Meirovich Y, Veinot J, de Bold M, Haddad H, Davies R, Masters R et al (2008) Relationship between natriuretic peptides and inflammation: proteomic evidence obtained during acute cellular cardiac allograft rejection in humans. J Heart Lung Transplant 27:31-37

51. Li C, Yu Q, Yu P, Yu T, Zhang Q, Lu S, Yu D (2014) Changes in liraglutide-induced body composition are related to modifications in plasma cardiac natriuretic peptides levels in obese type 2 diabetic patients. Cardiovasc Diabetol 13:36

52. Kim M, Platt M, Shibasaki T, Quaggin S, Backx P, Seino S et al (2013) GLP-1 receptor activation and Epac2 link atrial natriuretic peptide secretion to control of blood pressure. Nat Med 19:567-575

53. Maffei S, Del Ry S, Prontera C, Clerico A (2001) Increase in circulating levels of cardiac natriuretic peptides after hormone replacement therapy in postmenopausal women. Clin Sci 101:447-453

54. Clerico A, Ry Silvia D, Maffei S, Prontera C, Emdin M, Giannessi D (2002) The circulating levels of cardiac natriuretic hormones in healthy adults: effects of age and sex. Clin Chem Lab Med 40:371.

55. Hamada M, Shigetmatsu Y, Takezaki M, Ikeda S, Ogimoto A (2017) Plasma levels of atrial and brain natriuretic peptides in apparently healthy subjects: Effects of sex, age, and hemoglobin concentration. Int J Cardiol 228:599-604

56. Clerico A, Passino C, Emdin N (2011) When gonads talk to the heart sex hormones and cardiac endocrine function. J Am Coll Cardiol 58:627-628

57. Lam C, Cheng S, Choong K, Larson M, Murabito J, Newton-Cheh C et al (2011) Influence of sex and hormone status on circulating natriuretic peptides. J Am Coll Cardiol 58:618-626 
58. Goharian T, Gimsing A, Goetze, J, Faber J, Andersen L, Grontved A, Jeppesen J (2015) Mid-regional pro-atrial natriuretic peptide and blood pressure in adolescents: effect of gender and pubertal stage. Blood Press 24:347-352

59. Mahmoodzadeh S, Pham T, Kuehne A, Fielitz B, Dworatzek E, Kararigas G et al (2012) 17ß-Estradiol-induced interaction of ERa with NPPA regulates gene expression in cardiomyocytes. Cardiovasc Res 96:411-421

60. Sellitti D, Koles N, Mendonca M (2011) Regulation of C-type natriuretic peptide expression. Peptides 32:1964-1971

61. Khun M (2016) Molecular physiology of membrane guanylyl cyclase receptors. Physiol Rev 96:751-804

62. Stoupakis G, Klapholz M (2003) Natriuretic peptides: biochemistry, physiology, and therapeutic role in heart failure. Heart Dis 5:215-223

63. Yasoda A, Ogawa Y, Suda M, Tamura N, Mori K, Sakuma Y et al (1998) Natriuretic peptide regulation of endochondral ossification: evidence for possible roles of the $C$ type natriuretic peptide/guanylyl cyclase-B pathway. J Biochem 273:11695-11700

64. Misono K, Philo J, Arakawa T, Ogata C, Qiu Y, Ogawa H, Young H (2011) Structure, signaling mechanism and regulation of the natriuretic peptide receptor guanylate cyclase. FEBS J 278:1818-1829

65. Pandey K (2015) Endocytosis and Trafficking of Natriuretic Peptide Receptor-A: Potential Role of Short Sequence Motifs. Membranes (Basel) 5:253-287

66. Suga S, Nakao K, Hosoda K, Mukoyama M, Ogawa Y, Shirakami G et al (1992) Receptor selectivity of natriuretic peptide family, atrial natriuretic peptide, brain natriuretic peptide, and C-type natriuretic peptide. Endocrinology 130:229-239

67. Pandey K (1992) Kinetic analysis of internalization, recycling and redistribution of atrial natriuretic factor-receptor complex in cultured vascular smooth-muscle cells. Ligand-dependent receptor down-regulation. Biochem J 288:55-61

68. Matsukawa N, Grzesik W, Takahashi N, Pandey K, Pang S, Yamauchi M, et al (1999) The natriuretic peptide clearance receptor locally modulates the physiological effects of the natriuretic peptide system. PNAS 96:7403-7408

69. Kenny A, Bourne A, Ingram J (1993) Hydrolysis of human and pig brain natriuretic peptides, urodilatin, C-type natriuretic peptide and some C-receptor ligands by endopeptidase-24.11. Biochem J 1:83-88

70. Schling P, Löffer G (2002) Cross talk between adipose tissue cells: impact on pathophysiology. New Physiol Sci 17:99-104

71. Ralat L, Guo Q, Ren M, Funke T, Dickey D, Potter L, Tang W (2011) Insulindegrading enzyme modulates the natriuretic peptide-mediated signaling response. J Biol Chem 286:4670-4679

72. Brandt I, Lambeir A, Ketelslegers J, Vanderheyden M, Scharpé S, De Meester I (2006) Dipeptidyl-peptidase IV converts intact B-type natriuretic peptide into its desSerPro form. Clin Chem 52:82-87

73. Sarzani R, Dessi-Fulgheri P, Paci V, Espinosa E, Rappelli A (1996) Expression of natriuretic peptide receptors in human adipose and other tissues. J Endocrinol Invest 19:581-585

74. Bryan P, Smirnov D, Smolenski A, Feil S, Feil R, Hofmann F et al (2006) A sensitive method for determining the phosphorylation status of natriuretic peptide receptors: cGK-Ialpha does not regulate NPR-A. Biochemistry 45:1295-1303

75. Nagase M, Katafuchi T, Hirose S, Fujita T (1997) Tissue distribution and localization of natriuretic peptide receptor subtypes in stroke prone spontaneously hypertensive rats. J Hypertens 15:1235-1243 
76. Pivovarova O, Gögebakan Ö, Klöting N, Sparwasser A, Weickert M, Haddad I et al (2012) Insulin up-regulates natriuretic peptide clearance receptor expression in the subcutaneous fat depot in obese subjects: a missing link between CVD risk and obesity. J Clin Endocrinol Metab 94:E731-E739

77. Smith J, Fahrenkrug J, Jorgensen H, Christoffersen C, Goetze J (2015) Diurnal gene expression of lipolytic natriuretic peptide receptors in white adipose tissue. Endocr Connect 4:206-214

78. Martino T, Tata N, Belsham D, Chalmers J, Straume M, Lee P et al (2007) Disturbed diurnal rhythm alters gene expression and exacerbates cardiovascular disease with rescue by resynchronization. Hypertension 49:1104-1113

79. Goetze J, Jorgensen H, Sennels H, Fahrenkrug J (2012) Diurnal plasma concentrations of natriuretic propeptides in healthy young males. Clin Chem 58:789792

80. Goetze J, Georg B, Jorgensen H, Fahrenkrug J (2010) Chamber-dependent circadian expression of cardiac natriuretic peptides. Regul Pept 160: 140-145

81. Nakatsuji H, Maeda N, Hibuse T, Hiuge A, Hirata A, Kuroda Y et al (2010) Reciprocal regulation of natriuretic peptide receptors by insulin in adipose cells. Biochem Biophys Res Comm 392:100-105

82. Collins S (2014) A heart-adipose tissue connection in the regulation of energy metabolism. Nat Rev Endocrinol 10:157-163

83. Das S, Drazner M, Dries D, Vega G, Stanek H, Abdullah S et al (2005) Impact of body mass and body composition on circulating levels of natriuretic peptides: results from the Dallas Heart Study. Circulation 112:2163-2168

84. Cannone V, Boerrigter G, Cataliotti A, Costello-Boerrigter L, Olsen T, McKie P et al (2011) A genetic variant of the atrial natriuretic peptide gene is associated with cardiometabolic protection in the general community. J Am Coll Cardiol 58:629-636

85. Sugisawa T, Kishimoto I, Kokubo Y, Nagumo A, Makino H, Miyamoto Y, Yoshimasa $Y$ (2010) Visceral fat is negatively associated with B-type natriuretic peptide levels in patients with advanced type 2 diabetes. Diabetes Res Clin Pract 89:174-180

86. Buglioni A, Cannone V, Cataliotti A, Sangaralingham S, Heublein D, Scott C et al (2015) Circulating aldosterone and natriuretic peptides in the general community: relationship to cardiorenal and metabolic disease. Hypertension 65:45-53

87. Olsen M, Hansen T, Christensen M, Gustafsson F, Rasmussen S, Wachtell $K$ et al (2005) N-terminal pro brain natriuretic peptide is inversely related to metabolic cardiovascular risk factors and the metabolic syndrome. Hypertension 46:660-666

88. Taylor J, Christenson R, Rao K, Jorge M, Gottlieb S (2006) B-Type natriuretic peptide and $\mathrm{N}$-terminal pro $\mathrm{B}$-type natriuretic peptide are depressed in obesity despite higher left ventricular end diastolic pressures. Am Heart J 152:1071-1076

89. Grandi A, Laurita E, Selva E, Piantanida E, Imperiale D, Giovanella L et al (2004) Natriuretic peptides as markers of preclinical cardiac disease in obesity. Eur J Clin Invest 34:342-348

90. Abdulle A, Nagelkerke N, Adem A, Abouchacra S, Pathan J, Al-Rukhaimi M et al (2007) Plasma $\mathrm{N}$ terminal pro-brain natriuretic peptide levels and its determinants in a multi-ethnic population. J Hum Hypertens 21:647-653

91. Sarzani R, Strazzullo P, Salvi F, Iacone R, Pietrucci F, Siani A et al (2004) Natriuretic peptide clearance receptor alleles and susceptibility to abdominal adiposity. Obes Res 12:351-356

92. Cheng S, Fox C, Larson M, Massaro J, McCabe E, Khan A et al (2011) Relation of visceral adiposity to circulating natriuretic peptides in ambulatory individuals. Am J Cardiol 108:979-984 
93. Trevisan R, Fioretto P, Semplicini A, Opocher G, Mantero F, Rocco S et al (1990) Role of insulin and atrial natriuretic peptide in sodium retention in insulin-treated IDDM patients during isotonic volume expansion. Diabetes 39:289-298

94. Aboucharcra S, Baines A, Zinman B, Skorecki K, Logan A (1994) Insulin blunts the natriuretic action of atrial natriuretic peptide in hypertension. Hypertension 56:10541058

95. Neeland I, Winders B, Ayers C, Das S, Chang A, Berry J et al (2013) Higher natriuretic peptide levels associate with a favorable adipose tissue distribution profile. J Am Coll Cardiol 62:752-760

96. Hermann-Arnhof K, Hanusch-enserer U, Kaestenbauer T, Publig T, Dunky A, Rosen H et al (2005) N-terminal pro-B-type natriuretic peptide as an indicator of possible cardiovascular disease in severely obese individuals: comparison with patients in different stages of heart failure. Clin Chem 51:138-143

97. Thakur V, Richards R, Reisin E (2001) Obesity, hypertension, and the heart. Am J Med Sci 654:242-248

98. Burnett J, Granger J, Opgenorth T (1984) Effects of synthetic atrial natriuretic factor on renal function and renin release. Am J Physiol 247:F863-F866

99. Shi S, Nguyen H, Sharma G, Navar L, Pandey K (2001) Genetic disruption of atrial natriuretic peptide receptor-A alters renin and angiotensin II levels. Am J Physiol Renal Physiol 281:F665-F673

100. Asferg C, Andersen U, Linneberg A, Hedley P, Christiansen M, Goetze J, Jeppesen J (2017) Serum proatrial natriuretic peptide does not increase with higher systolic blood pressure in obese men. Heart 103:154-158

101. Kurukulasuriya L, Stas S, Lastra G, Manrique C, Sowers J (2011) Hypertension in obesity. Med Clin North Am 95:903-917

102. Gruden G, Landi A, Bruno G (2014) Natriuretic peptides, heart, and adipose tissue: new findings and future developments for diabetes research. Diabetes Care 37:28992908

103. Hammerer-Lercher A, Ludwig W, Falkensammer G, Müller S, Neubauer E, Puschendorf B et al (2004) Natriuretic peptides as markers of mild forms of left ventricular dysfunction: effects of assays on diagnostic performance of markers. Clin Chem 50:1174-1183

104. Wong C, O'Moore-Sullivan T, Leano R, Byrne N, Beller E, Marwick T (2004) Alterations of left ventricular myocardial characteristics associated with obesity. Circulation 110:3081-3087

105. Jujic A, Nilsson P, Persson M, Holst J, Torekov S, Lyssenko V et al (2016) Atrial Natriuretic Peptide in the High Normal Range Is Associated With Lower Prevalence of Insulin Resistance. J Clin Endocrinol Metab 414:1372-1380

106. Brutsaert E, Biggs M, Delaney J, Djoussé L, Gottdiener J, Ix J et al (2016) Longitudinal assessment of $\mathrm{N}$-terminal pro-B-type natriuretic peptide and risk of diabetes in older adults: The cardiovascular health study. Metabolism 65:1489-1497

107. Kim F, Biggs M, Kizer J, Brutsaert E, de Filippi C, Newman A et al (2017) Brain natriuretic peptide and insulin resistance in older adults. Diabet Med 34:235-238

108. Kroon M, van den Hurk K, Alssema M, Kamp O, Stehouwer C, Henry R (2012) Prospective associations of $B$-type natriuretic peptide with markers of left ventricular function in individuals with and without type 2 diabetes: an 8-year follow-up of the Hoorn Study. Diabetes Care 35:2510-2514

109. Everett B, Cook N, Chasman D, Magnone M, Bobadilla M, Rifai N et al (2013) Prospective evaluation of $B$-type natriuretic peptide concentrations and the risk of type 2 diabetes in women. Clin Chem 59:557-565 
110. Pfister R, Sharp S, Luben R, Welsh P, Barroso I, Salomaa V et al (2011) Mendelian randomization study of B-type natriuretic peptide and type 2 diabetes: evidence of causal association from population studies. PLoS Med 8:e1001112

111. Cannone V, Cefalu A, Noto D, Scott C, Bailey K, Cavera G et al (2013) The atrial natriuretic peptide genetic variant rs5068 is associated with a favorable cardiometabolic phenotype in a Mediterranean population. Diabetes Care 36:28502856

112. Heinisch B, Vila G, Resl M, Riedl M, Dieplinger B, Mueller T et al (2012) B-type natriuretic peptide (BNP) affects the initial response to intravenous glucose: a randomised placebo-controlled cross-over study in healthy men. Diabetologia 55:1400-1405

113. Jujic A, Nilsson P, Engström G, Hedblad B, Melander O, Magnusson M (2014) Atrial natriuretic peptide and type 2 diabetes development--biomarker and genotype association study. PLoS One 9:e89201

114. Meirhaeghe A, Sandhu M, McCarthy M, de Groote P, Cottel D, Arveiler D et al (2007) Association between the T-381C polymorphism of the brain natriuretic peptide gene and risk of type 2 diabetes in human populations. Human Molecular Genetics 16:1343-1350

115. Cannone V, Huntley B, Olson T, Heublein D, Scott C, Bailey K et al (2013) Atrial natriuretic peptide genetic variant rs5065 and risk for cardiovascular disease in the general community: a 9-year follow-up study. Hypertension 62:860-865

116. Maack T, Suzuki M, Almeida F, Nussenzveig D, Scarborough R, McEnroe G, Lewicki J (1987) Physiological role of silent receptors of atrial natriuretic factor. Science 238:675-678

117. Bordicchia M, Ceresiani M, Pavani M, Minardi D, Polito M, Wabitsch M et al (2016) Insulin/glucose induces natriuretic peptide clearance receptor in human adipocytes: a metabolic link with the cardiac natriuretic pathway. Am J Physiol Regul Integr Comp Physiol 311:R104-R114

118. Halbrik M, Norrelund H, Moller N, Schmitz O, Botker H, Wiggers H (2010) Short-term changes in circulating insulin and free fatty acids affect Nt-pro-BNP levels in heart failure patients. Int J Cardiol 144:140-142

119. Coué M, Badin P, Vila I, Laurens C, Louche K, Marquès M et al (2015) Defective Natriuretic Peptide Receptor Signaling in Skeletal Muscle Links Obesity to Type 2 Diabetes. Diabetes 64:4033-4045

120. Rydén M, Backdahl J, Petrus P, Thorell A, Gao H, Coue M et al (2016) Impaired atrial natriuretic peptide-mediated lipolysis in obesity. Int J Obes 40:714-20

121. Sarzani R, Paci V, Zingaretti C, Pierleoni C, Cinti S, Cola G et al (1995) Fasting inhibits natriuretic peptides clearance receptor expression in rat adipose tissue. J Hypertens 13:1241-1246

122. Miyashita $K$, Itoh $H$, Tsujimoto $H$, Tamura N, Fukunaga $Y$, Sone $M$ et al (2009) Natriuretic peptides/cGMP/cGMP-dependent protein kinase cascades promote muscle mitochondrial biogenesis and prevent obesity. Diabetes 58:2880-2892

123. Standeven K, Hess K, Carter A, Rice G, Cordell P, Balmforth A et al (2011) Neprilysin, obesity and the metabolic syndrome. Int J Obes 35:1031-1040

124. Minami J, Nishikimi T, Matsuoka H (2004) Plasma brain natriuretic peptide and $\mathrm{N}$ terminal proatrial natriuretic peptide levels in obese patients: a cause or result of hypertension. Circulation 110:e76

125. Mizuno Y, Harada E, Katoh D, Kashiwagi Y, Morikawa Y, Nakagawa, H et al (2013) Cardiac production of $\mathrm{B}$-type natriuretic peptide is inversely related to the plasma 
level of free fatty acids in obese individuals - possible involvement of the insulin resistance. Endocr J 60:87-95

126. Plante E, Menaouar A, Danalache B, Broderick T, Jankowski M, Gutkowska J (2014) Treatment with brain natriuretic peptide prevents the development of cardiac dysfunction in obese diabetic db/db mice. Diabetologia 57:1257-1267

127. Cabiati M, Raucci S, Liistro T, Belcastro E, Prescimone T, Caselli C et al (2013) Impact of obesity on the expression profile of natriuretic peptide system in a rat experimental model. PLoS One 8:e72959

128. Maisal A (2002) B-type natriuretic peptide levels: diagnostic and prognostic in congestive heart failure: what's next. Circulation 105:2328-2331

129. Sengenes C, Berlan M, De Glisezinski I, Lafontan M, Galitzky J (2000) Natriuretic peptides: a new lipolytic pathway in human adipocytes. FASEB J 14:1345-1351

130. Sengenès $C$, Zakaroff-Girard A, Moulin A, Berlan $M$, Bouloumié $A$, Lafontan $M$ et al (2002). Natriuretic peptide-dependent lipolysis in fat cells is a primate specificity. Am J Physiol Regul Integr Comp Physiol 283, R257-R265

131. Sengenes C, Bouloumie A, Hauner H, Berlan M, Busse R, Lafontan M et al (2003) Involvement of a cGMP-dependent pathway in the natriuretic peptide-mediated hormone-sensitive lipase phosphorylation in human adipocytes. J Biol Chem 278:48617-48626

132. Birkenfeld A, Boschmann M, Moro C, Adams F, Heusser K, Franke G et al (2005) Lipid mobilization with physiological atrial natriuretic peptide concentrations in humans. J Clin Endocrinol Metab 90:3622-3628

133. Ahmadian M, Abbott M, Tang T, Hudak C, Kim Y, Bruss M et al (2011) Desnutrin/ATGL is regulated by AMPK and is required for a brown adipose phenotype. Cell Metab 13:739-748

134. Zimmerman R, Straus J, Haemmerle G, Schoiswohl G, Birner-Gruenberger R, Riederer $M$ et al (2004) Fat mobilization in adipose tissue is promoted by adipose triglyceride lipase. Science 306:1383-1386

135. Galitzky J, Sengenes C, Thalamas C, Marques M, Senard J, Lafontan M, Berlan M (2001) The lipid-mobilizing effect of atrial natriuretic peptide is unrelated to sympathetic nervous system activation or obesity in young men. J Lipid Res 42:536544

136. Birkenfeld A, Boschmann M, Moro C, Adams F, Heusser K, Tank J et al (2006) Betaadrenergic and Atrial Natriuretic Peptide Interactions on Human Cardiovascular and Metabolic Regulation. J Clin Endocrinol Metab 91:5069-5075

137. Moro C, Galitzky J, Sengenes C, Crampes F, Lafontan M, Berlan M (2004) Functional and pharmacological characterization of the natriuretic peptide-dependent lipolytic pathway in human fat cells. J Pharmacol Exp Therp 308:984-992

138. Birkenfeld A, Budziarek P, Boschmann M, Moro C, Adams F, Franke G et al (2008) Atrial natriuretic peptide induces postprandial lipid oxidation in humans. Diabetes 57:3199-3204

139. Moro C, Crampes F, Sengenes C, De Glisezinski I, Galitzky J, Thalamas C et al (2004) Atrial natriuretic peptide contributes to physiological control of lipid mobilization in humans. FASEB J 18:908-910

140. Langin D, Dicker A, Tavernier G, Hoffstedt J, Mairal A, Rydén M et al (2005) Adipocyte lipases and defect of lipolysis in human obesity. Diabetes 54:3190-3197

141. Rydén M, Jocken J, van Harmelen V, Dicker A, Hoffstedt J, Wirén M et al (2007) Comparative studies of the role of hormone-sensitive lipase and adipose triglyceride lipase in human fat cell lipolysis. Am J Physiol Endocrinol Metab 292:E1847-E18455 
142. Jocken J, Blaak E (2008) Catecholamine-induced lipolysis in adipose tissue and skeletal muscle in obesity. Physiol Behav 94:219-230

143. Dicker A, Aström G, Wahlén K, Hoffstedt J, Näslund E, Wirén M et al (2009) Primary differences in lipolysis between human omental and subcutaneous adipose tissue observed using in vitro differentiated adipocytes. Horm Metab Res 41:350-355

144. Endre T, Mattiasson I, Berglund G, Hulthén U (1994) Insulin and renal sodium retention in hypertension-prone men. Hypertension 23:313-319

145. Moro C, Polak J, Richterova B, Sengenès C, Pelikanova T, Galitzky J et al (2005) Differential regulation of atrial natriuretic peptides and adrenergic receptor dependent lipolytic pathways in human adipose tissue. Metabolism 54:122-131

146. Kovacova Z, Tharp W, Liu D, Wei W, Xie H, Collins S et al (2016) Adipose tissue natriuretic peptide receptor expression is related to insulin sensitivity in obesity and diabetes. Obesity 24:820-8

147. Fonseca $V$ (2003) Management of diabetes mellitus and insulin resistance in patients with cardiovascular disease. Am J Cardiol 92:50J-60J

148. Arora P, Wu C, Hamid T, Arora G, Agha O, Allen K et al (2016) Acute Metabolic Influences on the Natriuretic Peptide System in Humans. J Am Coll Cardiol 67:804812

149. Arora P, Wu C, Khan A, Bloch D, Davis-Dusenbery B, Ghorbani A et al (2013) Atrial natriuretic peptide is negatively regulated by microRNA-425. J Clin Invest 123:33783382

150. Tsukamoto O, Fujita M, Kato M, Yamazaki S, Asono Y, Ogai A et al (2009) Natriuretic peptides enhance the production of adiponectin in human adipocytes and in patients with chronic heart failure. J Am Coll Cardiol 53:2070-2077

151. Birkenfeld A, Boschmann M, Engeli S, Moro C, Arafat A, Luft F, Jordan J (2012) Atrial natriuretic peptide and adiponectin interactions in man. PLoS One 7:e43238

152. Wannamethee S, Welsh P, Whincup P, Sawar N, Thomas M, Gudnarsson V, Sattar N (2011) High adiponectin and increased risk of cardiovascular disease and mortality in asymptomatic older men: does NT-proBNP help to explain this association. Eur J Cardiovasc Prev Rehabil 18:65-71

153. Nakanishi K, Nishida M, Yamamoto R, Koseki M, Moriyama T, Yamauchi-Takihara K (2016) Association between $\mathrm{N}$-terminal pro-brain natriuretic peptide and adiponectin in healthy Japanese men. Clin Chim Acta 460:138-141

154. Moro C, Klimcakova E, Lolmède K, Berlan M, Lafontan M, Stich V et al (2007) Atrial natriuretic peptide inhibits the production of adipokines and cytokines linked to inflammation and insulin resistance in human subcutaneous adipose tissue. Diabetologia 50:1038-1047

155. Van Marken Lichtenbelt W, Vanhommerig J, Smulders N, Drossaerts J, Kemerink G, Bouvy $\mathrm{N}$ et al (2009) Cold-activated brown adipose tissue in healthy men. $\mathrm{N}$ Engl J Med 360:1500-1508

156. Virtanen K, Lidell M, Orava J, Heglind M, Westergren R, Niemi T et al (2009) Functional brown adipose tissue in healthy adults. N Engl J Med 360:1518-1525

157. Cypess A, Lehman S, Williams G, Tal I, Rodman D, Goldfine A et al (2009) Identification and importance of brown adipose tissue in adult humans. $\mathrm{N}$ Engl J Med 360:1509-1517

158. Schrauwen $P$, van Marken Lichtenbelt W (2016) Combatting type 2 diabetes by turning up the heat. Diabetologia 59:2269-2279

159. Cannon B, Nedergaard J (2004) Brown adipose tissue: function and physiological significance. Physiol Rev 84:277-359 
160. Feldmann H, Golozoubova V, Cannon B, Nedergaard J (2009) UCP1 ablation induces obesity and abolishes diet-induced thermogenesis in mice exempt from thermal stress by living at thermoneutrality. Cell Metab 9:203-209

161. Park J, Hur W, Lee S (2015) Intricate Transcriptional Networks of Classical Brown and Beige Fat Cells. Front Endocrinol 6:124

162. Souza S, Chau M, Yang Q, Gauthier M, Clairmont K, Wu Z et al (2011) t Atrial natriuretic peptide regulates lipid mobilization and oxygen consumption in human adipocytes by activating AMPK. Biochm Biophys Res Comm 410:398-403

163. Nishizawa H, Matsuda M, Yamada Y, Kawai K, Suzuki E, Makishima M et al (2004) Musclin, a novel skeletal muscle-derived secretory factor. J Biol Chem 279:1939119395

164. Subbotina E, Sierra A, Zhu Z, Gao Z, Koganti S, Reyes S et al (2015) Musclin is an activity-stimulated myokine that enhances physical endurance. Proc Natl Acad Sci USA 112:16042-16047

165. Gu N, Guo Q, Mao K, Hu H, Jin S, Zhou Y et al (2015) Palmitate increases musclin gene expression through activation of PERK signaling pathway in $\mathrm{C} 2 \mathrm{C} 12$ myotubes. Biochem Biophys Res Commun 467:521-526

166. Yu J, Zheng J, Liu X, Feng Z, Zhang X, Cao L, Zhou Z (2016) Exercise improved lipid metabolism and insulin sensitivity in rats fed a high-fat diet by regulating glucose transporter 4 (GLUT4) and musclin expression. Braz J Med Biol Res 49:e5129

167. Liu Y, Huo X, Pang X, Zong Z, Meng X, Liu G (2008) Musclin inhibits insulin activation of $A$ kt/protein kinase $B$ in rat skeletal muscle. J Int Med Res 36:496-504

168. Birkenfeld A, Shulman G (2014) Nonalcoholic fatty liver disease, hepatic insulin resistance, and type 2 diabetes. Hepatology 59:713-723

169. Gaggini M, Morelli M, Buzzigoli E, DeFronzo R, Bugianese E, Gastaldelli A (2013) Non-alcoholic fatty liver disease (NAFLD) and its connection with insulin resistance, dyslipidemia, atherosclerosis and coronary heart disease. Nutrients 5:1544-1560

170. Day C (2002) Pathogenesis of steatohepatitis. Best Pract Res Clin Gastroenterology $16: 663-678$

171. Sanchez O, Lazo-Elizondo M, Zeb I, Tracy R, Bradley R, Duprez D et al (2016) Computerized tomography measured liver fat is associated with low levels of $\mathrm{N}$ terminal pro-brain natriuretic protein (NT-proBNP) - Multi-Ethnic Study of Atherosclerosis. Metabolism 65:728-735

172. Lazo M, Rubin J, Clark J, Coresh J, Schneider A, Ndumele C et al (2015) The Association of Liver Enzymes with Biomarkers of Subclinical Myocardial Damage and Structural Heart Disease. J Hepatol 62:841-847

173. Vollmar A, Paumgartner G, Gerbes A (1997) Differential gene expression of the three natriuretic peptides and natriuretic peptide receptor subtypes in human liver. Gut 40:145-150

174. Bilzer M, Jaeschke H, Vollmar A, Paumgartner G, Gerbes A (1999) Prevention of Kupffer cell-induced oxidant injury in rat liver by atrial natriuretic peptide. Am J Physiol 276:G1137-G1144

175. Kiemer A, Baron A, Gerbes A, Bilzer M, Vollmar A (2002) The atrial natriuretic peptide as a regulator of Kupffer cell functions. Shock 17:365-371

176. Rashed H, Nair B, Patel T (1992) Regulation of hepatic glycolysis and gluconeogenesis by atrial natriuretic peptide. Arch Biochem Biophys 298:640-645

177. Lutz S, Hennige A, Feil S, Peter A, Gerling A, Machann J (2011) Genetic ablation of cGMP-dependent protein kinase type I causes liver inflammation and fasting hyperglycemia. Diabetes 60:1566-1576 
178. Arbin V, Claperon N, Fournié-Zaluski M, Roques B, Peyroux J (2001) Acute effect of the dual angiotensin-converting enzyme and neutral endopeptidase 24-11 inhibitor mixanpril on insulin sensitivity in obese Zucker rat. Br J Pharmacol 133:495-502

179. Arbin V, Claperon N, Fournié-Zaluski M, Roques B, Peyroux J (2003) Effects of dual angiotensin-converting enzyme and neutral endopeptidase 24-11 chronic inhibition by mixanpril on insulin sensitivity in lean and obese Zucker rats. J Cardiovasc Pharmacol 41:254-264

180. Uehlinger D, Weidmann P, Gnädinger M, Hasler L, Bachmann C, Shaw S et al (1986) Increase in circulating insulin induced by atrial natriuretic peptide in normal humans. J Cardiovasc Pharmacol 8:1122-1129

181. Ferrari P, Shaw S, Riesen W, Weidmann P (1992) Plasma insulin during physiological and pathophysiological changes in atrial natriuretic factor. Eur J Clin Pharmacol 42:453-455

182. Ropero A, Soriano S, Tudurí E, Marroquí L, Téllez N, Gassner B et al (2010) The atrial natriuretic peptide and guanylyl cyclase-a system modulates pancreatic betacell function. Endocrinology 151:3665-3674

183. You H, Laychock S (2009) Atrial natriuretic peptide promotes pancreatic islet betacell growth and Akt/Foxo1a/cyclin D2 signaling. Endocrinology 150:5455-5465

184. Moro C, Polak J, Hejnova J, Klimcakova E, Crampes F, Stich V et al (2006) Atrial natriuretic peptide stimulates lipid mobilization during repeated bouts of endurance exercise. Am J Physiol Endocrinol Metab 290:E864-E869

185. Moro C, Pillard F, de Glisezinski I, Klimcakova E, Crampes F, Thalamas C et al (2008) Exercise-induced lipid mobilization in subcutaneous adipose tissue is mainly related to natriuretic peptides in overweight men. Am J Physiol Endocrinol Metab 295:E505E513

186. Moro C, Pillard F, De Glisezinski I, Harant I, Rivière D, Stich V et al (2005) Training Enhances ANP Lipid-Mobilizing Action in Adipose Tissue of Overweight Men. Medicine \& Science in Sports \& Exercise 37:1126-32

187. Barletta G, Stefani L, Del Bene R, Fronzaroli C, Vecchiarino S, Lazzeri C et al (1998) Effects of exercise on natriuretic peptides and cardiac function in man. Int J Cardiol 65:217-225

188. Kjaer A, Appel J, Hildebrandt P, Petersen C (2004) Basal and exercise-induced neuroendocrine activation in patients with heart failure and in normal subjects. Eur J Heart Fail 6:29-39

189. Mandroukas A, Metaxas T, Heller J, Vamvakoudis E, Christoulas K, Riganas C et al (2011) The effect of different exercise-testing protocols on atrial natriuretic peptide. Clin Physiol Funct Imaging 31:5-10

190. Steele I, McDowell G, Moore A, Campbell N, Shaw C, Buchanan K et al (1997) Responses of atrial natriuretic peptide and brain natriuretic peptide to exercise in patients with chronic heart failure and normal control subjects. Eur J Clin Invest 27:270-276

191. Kato M, Kinugawa T, Ogino K, Endo A, Osaki S, Igawa O et al (2000) Augmented response in plasma brain natriuretic peptide to dynamic exercise in patients with left ventricular dysfunction and congestive heart failure. J Intern Med 248:309-315

192. Engelmann M, Nieman L, Kanstrup I, Skagen K, Godtfredsen J (2005) Natriuretic peptide response to dynamic exercise in patients with atrial fibrillation. Int J Cardiol 105:31-39

193. Tanaka M, Ishizaka Y, Ishiyama Y, Kato J, Kida O, Kitamura K et al (1995) Exerciseinduced secretion of brain natriuretic peptide in essential hypertension and normal subjects. Hypertens Res 18:159-166 
194. Haufe S, Kaminski J, Utz W, Haas V, Mähler A, Daniels M et al (2015) Differential response of the natriuretic peptide system to weight loss and exercise in overweight or obese patients. J Hypertens 33:1458-1464

195. Bordbar S, Bigi M, Aslani A, Rahimi E, Ahmadi N (2012) Effect of endurance and strength exercise on release of brain natriuretic peptide. J Cardiovasc Dis Res 3:2225

196. Beltran Valls M, Dimauro I, Brunelli A, Tranchita E, Ciminelli E, Caserotti P et al (2014) Explosive type of moderate-resistance training induces functional, cardiovascular, and molecular adaptations in the elderly. Age 36:759-772

197. Lu J, Pan S (2016) Elevated C-type natriuretic peptide elicits exercise preconditioning-induced cardioprotection against myocardial injury probably via the up-regulation of NPR-B. J Physiol Sci [Epub ahead of print]

198. Liu Y, Li J, Zhang Z, Tang Y, Chen Z, Wang Z (2013) Effects of exercise intervention on vascular endothelium functions of patients with impaired glucose tolerance during prediabetes mellitus. Exp Ther Med 5:1559-1565

199. Jenkins N, Padilla J, Rector R, Laughlin M (2013) Influence of regular physical activity and caloric restriction on $\beta$-adrenergic and natriuretic peptide receptor expression in retroperitoneal adipose tissue of OLETF rats. Exp Physiol 98:1576-1584

200. Moro C, Pasarica M, Elkind-Hirsch K, Redman L (2009) Aerobic exercise training improves atrial natriuretic peptide and catecholamine-mediated lipolysis in obese women with polycystic ovary syndrome. J Clin Endocrinol Metab 94:2579-2586

201. Fenzl M, Schnizer W, Aebli N, Schlegel C, Villiger B, Disch A et al (2013) Release of ANP and fat oxidation in overweight persons during aerobic exercise in water. Int J Sports Med 34:795-799

202. Endlich P, Firmes L, Goncalves W, Gouveau S, Moyses M et al (2011) Involvement of the atrial natriuretic peptide in the reduction of arterial pressure induced by swimming but not by running training in hypertensive rats. Peptides 32:1706-1712

203. Menshikova E, Ritov V, Toledo F, Ferrell R, Goodpaster B, Kelley D (2005) Effects of weight loss and physical activity on skeletal muscle mitochondrial function in obesity. Am J Physiol Endocrinol Metab 288:E818-E825

204. St Peter J, Hartley G, Murakami M, Apple F (2006) B-type natriuretic peptide (BNP) and $\mathrm{N}$-terminal pro-BNP in obese patients without heart failure: relationship to body mass index and gastric bypass surgery. Clin Chem 52:680-685

205. Changchien E, Ahmed S, Betti F, Higa J, Kiely K, Hernandez-Boussard T, Morton J (2011) B-type natriuretic peptide increases after gastric bypass surgery and correlates with weight loss. Surg Endosc 25:2338-2343

206. Chainani-Wu N, Weidner G, Purnell D, Frenda S, Merritt-Worden T, Kemp C et al (2010) Relation of B-type natriuretic peptide levels to body mass index after comprehensive lifestyle changes. Am J Cardiol 105:1570-1576

207. Chen-Tournoux A, Khan A, Baggish A, Castro V, Semigran M, McCabe E et al (2010) Effect of weight loss after weight loss surgery on plasma $\mathrm{N}$-terminal pro-B-type natriuretic peptide levels. Am J Cardiol 106:1450-1455

208. Abrahamsson N, Engström B, Sundbom M, Karlsson F (2013) Gastric bypass surgery elevates NT-ProBNP levels. Obes Surg 23:1421-1426

209. Kistorp C, Bliddal H, Goetze J, Christensen R, Faber J (2014) Cardiac natriuretic peptides in plasma increase after dietary induced weight loss in obesity. BMC Obes $1: 24$

210. Hanusch-Enserer U, Hermann K, Cauza E, Spak M, Mähr B, Dunky A et al (2003) Effect of gastric banding on aminoterminal pro-brain natriuretic peptide in the morbidly obese. Obes Res 11:695-698 
211. Minami J, Nishikimi T, Ishimitsu T, Makino Y, Kawano Y, Takishita S et al (2000) Effect of a hypocaloric diet on adrenomedullin and natriuretic peptides in obese patients with essential hypertension. J Cardiovasc Pharmacol 36:S83-S86

212. Gabrielsen A, Omland T, Brokner M, Fredheim J, Jordan J, Lehmann S et al (2016) The effect of surgical and non-surgical weight loss on $\mathrm{N}$-terminal pro-B-type natriuretic peptide and its relation to obstructive sleep apnea and pulmonary function. BMC Res Notes 9:440 



\section{Chapter 3}

\section{Attenuated atrial natriuretic peptide- mediated lipolysis in subcutaneous adipocytes of obese type 2 diabetic men}

Kenneth Verboven, Dominique Hansen, Cedric Moro, Bert O Eijnde, Nicole Hoebers, Joep Knol, Wim Bouckaert, Anne Dams, Ellen E Blaak, Johan WE Jocken 


\section{Abstract}

Aims/hypothesis Catecholamines and atrial natriuretic peptide (ANP) are major regulators of adipocyte lipolysis. Although obesity is characterized by catecholamine resistance in subcutaneous adipose tissue (SCAT), data on ANP lipolytic response and sensitivity in different adipose tissue depots of metabolically distinct humans are scarce.

Methods Ex vivo catecholamine- and ANP-induced lipolysis was investigated in adipocytes derived from SCAT and visceral (VAT) depot of lean $(n=13)$ and obese men, with $(n=11)$ or without $(n=18)$ type 2 diabetes (HbA1c < or $\geq 6.5 \%$ ). Underlying molecular mechanisms were examined by looking at functional receptors in the NP signaling pathway on mRNA and protein level.

Results Maximal ANP- and catecholamine-induced lipolysis in SCAT was blunted in obese type 2 diabetic compared with age-matched lean men whilst non-diabetic obese subjects showed intermediate responses. This blunted ANPmediated lipolytic response was accompanied by lower mRNA and protein expression of the type-A natriuretic peptide (NP) receptor and higher mRNA but reduced protein expression of the scavenging type-C receptor. Maximal ANPinduced lipolysis was lower in VAT compared to SCAT but not different between groups.

Conclusions/interpretation Collectively, our data show that both ANP- and catecholamine-mediated lipolysis is attenuated in SCAT of obese men with type 2 diabetes, and might be partially explained by NP receptor defects. Therefore, improving maximal ANP responsiveness in adipose tissue might be a potential novel strategy to improve obesity-associated metabolic complications. 


\section{Introduction}

Adipose tissue (AT) dysfunction is a commonly observed feature of metabolic dysfunction in obesity ${ }^{1}$. Since the subcutaneous adipose tissue (SCAT) has only a limited expandability, ectopic lipid accumulation contributes to the onset or worsening of obesity-related insulin resistance ${ }^{2,3}$. As obesity and associated insulin resistance are important risk factors for the development of cardiometabolic diseases like type 2 diabetes mellitus ${ }^{4}$, it is imperative to investigate AT function in obese insulin resistant subjects with and without type 2 diabetes. An important characteristic when examining AT function is the capacity to break down intracellular stored lipids, a process called lipolysis ${ }^{5}$. This hydrolysis of triacylglycerol (TAG) stored in adipocytes involves different lipases (i.e. adipose triglyceride lipase (ATGL), hormone sensitive lipase (HSL) and monoacylglycerol lipase (MGL)), and their activity is regulated by several hormones including catecholamines, insulin and natriuretic peptides (NP) ${ }^{6}$. In the obese state, SCAT adipocytes show an increased 7 , unchanged 8 or decreased ${ }^{9,10}$ basal (non-stimulated) fasting lipolysis (depending on the degree of insulin resistance and hyperinsulinemia or method of expression), while catecholamine resistance of SCAT lipolysis is most commonly observed $7,10,11$. An increased catecholamine sensitivity has been reported for visceral AT (VAT) ${ }^{12,13}$. Differences at receptor and post-receptor signaling may contribute to lipolytic differences between depots ${ }^{14-17}$.

Natriuretic peptides, including atrial, B- and C-type natriuretic peptide, display primate-specific effects in human white adipocytes. Of all NP family members, Sengenes et al. showed ANP to be the most potent activator of lipolysis in human adipocytes ${ }^{18}$. Moreover, limited data are available about the role of atrial natriuretic peptide (ANP) in human AT lipolysis, of which an overview is shown in Table 3. In 2000 the discovery of the in vitro lipolytic potency ${ }^{18}$ and later the ability of increasing plasma non-esterified fatty acids (NEFA) upon intravenous infusion of ANP, suggested its contribution to an enhanced lipid catabolism in humans ${ }^{19-21}$. Until today, only one in vivo study indicated an attenuated ANP-induced lipolysis in the SCAT of young overweight/obese men and women ${ }^{22}$. However, data on depot-specific differences in ANP sensitivity in obesity are scarce as only two studies suggested ANP-mediated lipolysis to be 
similar in SCAT and VAT of morbidly obese subjects ${ }^{23,24}$. However, these studies did not include lean subjects, nor obese subjects with type 2 diabetes. The lipolytic effect of ANP is determined by binding to the NP receptor type-A (NPRA) which stimulates cGMP-dependent activation (phosphorylation) of the lipiddroplets associated protein perilipin 1 (PLIN1) and HSL ${ }^{18,20,25}$. Next to NPRA, ANP-mediated lipolysis is dependent on NP clearance, predominantly mediated by the NP receptor type C (NPRC) ${ }^{26-28}$ which is abundantly present in human white AT ${ }^{29,30}$. An increased expression of NPRC in the SCAT of obese hypertensive patients compared to non-obese and normotensive subjects has been observed ${ }^{31}$, which is associated with a reduced NP availability in these patients and possibly a lower NP-mediated lipid mobilization. In addition, insulin has been shown to regulate NPRC expression in human adipocytes ${ }^{24,30,32}$. Yet, the possible relevance of insulin-induced upregulation of NPRC and the effect of obesity per se on ANP-mediated AT lipolysis remains unclear and needs further investigation. (Patho)physiological variations in different components of the ANP-mediated lipolytic pathway should be investigated thoroughly, especially under metabolically compromised conditions like obesity or type 2 diabetes ${ }^{33}$.

In the present study, the aim was to investigate whether ex vivo ANP-mediated lipolysis is altered in adipocytes derived from SCAT or VAT of obese subjects with or without type 2 diabetes, compared to age-matched lean men. Furthermore, the underlying molecular mechanism was examined by looking at functional receptors in the NP signaling pathway both at transcriptional (mRNA) and translational (protein) level. 


\section{Methods}

Subjects - For this study, male subjects who were scheduled to undergo abdominal or bariatric surgery were recruited. The decision to undergo surgery was made independently of this research protocol, between patient and physician. The study sample size was based on previous studies assessing AT lipolysis in non-obese and obese subjects ${ }^{11,12}$. The control group consisted of 13 lean male subjects (age $51 \pm 5$ years; BMI $23.9 \pm 1.1 \mathrm{~kg} / \mathrm{m}^{2}$; HbA1c $5.3 \pm$ $0.2 \%$ ( $35 \pm 2 \mathrm{mmol} / \mathrm{mol})$ ). The obese group was composed of 18 male subjects without type 2 diabetes (age $47 \pm 5$ years; BMI $38.3 \pm 3.9 \mathrm{~kg} / \mathrm{m}^{2} ; \mathrm{HbA} 1 \mathrm{c} 5.5 \pm$ $0.3 \%(37 \pm 3 \mathrm{mmol} / \mathrm{mol})$ ) and 11 male subjects with type 2 diabetes (age $51 \pm$ 7 years; BMI $38.1 \pm 3.7 \mathrm{~kg} / \mathrm{m}^{2}$; HbA1c $7.2 \pm 0.9 \%(56 \pm 10 \mathrm{mmol} / \mathrm{mol})$ ). Presence of type 2 diabetes was defined as glycated hemoglobin (HbA1c) $\geq$ $6.5 \%(45 \mathrm{mmol} / \mathrm{mol})$ or the use of glucose lowering medication based on a clinical diagnosis (on average 2.5 years of diagnosis, ranging from newly diagnosed to 6 years). Clinical characteristics and use of glucose/lipid lowering and anti-hypertensive medication of the subjects included in the study are summarized in Table 1. Major exclusion criteria were presence of a history of heart, lung or kidney disease and/or presence of endocrine anomalies. The study protocol was approved by the Medical Ethical Committee of Jessa Hospital in accordance with the Declaration of Helsinki, and all subjects gave their written informed consent before participating in the study (ClinicalTrial.gov accession number NCT02598544).

Anthropometric measurements and blood sampling - Body weight, height, waist/hip circumference and blood pressure were determined the morning of surgery (between 7-10 am). Fat and lean body mass were estimated by bioelectrical impedance analyses (Bodystat 1500; Bodystat Ltd., Isle of Man, U.K.). Fasting venous blood samples were collected after an overnight fast (at least 10 hours) for the measurement of plasma ANP, glucose, triglycerides, NEFA, serum insulin and HbA1c. Insulin sensitivity was assessed by the homeostasis model assessment index for insulin resistance (HOMA-IR), calculated from fasting glucose and insulin, according to the formula: fasting insulin $(\mu \mathrm{U} / \mathrm{l}) *$ fasting glucose $(\mathrm{mmol} / \mathrm{l}) / 22.5^{34}$. Plasma levels of ANP were measured using the RayBio ANP Enzyme Immunoassay Kit (Tebu-Bio, Boechout, 
Belgium). Plasma glucose was measured by the glucose oxidase method using a AU2700 analyzer (Beckman Coulter, Brea, CA, USA). Serum insulin was assessed by immunoassay (ADVIA Centaur Insulin IRI; Siemens Medical Solutions Diagnostics, Tarrytown, NY, USA). Plasma NEFA (NEFA C kit; Wako Chemicals, Neuss, Germany) and TAG (Sigma, St. Louis, MO) were determined by an enzymatic colorimetric quantification on a COBAS FARA centrifugal spectrophotometer (Roche, Diagnostica, Basel, Switzerland). HbA1C was assessed by high performance liquid chromatography using a HA-8160 Hi-Auto A1C analyzer (Menarini, Zaventem, Belgium).

Adipose tissue biopsies - After an overnight fast (at least 10 hours) abdominal subcutaneous (SCAT) and omentus majus (VAT) AT biopsies were collected from all participants during abdominal surgery (under general anesthesia). For each AT depot, approximately 1-5 g AT was collected. Tissue was immediately brought to the laboratory for further processing or frozen in liquid nitrogen for gene and protein expression analyses.

Adipocyte isolation and lipolysis experiments - Mature adipocytes were obtained by collagenase digestion of AT fragments in Dulbecco's Modified Eagle's Medium(DMEM)-Ham's F12 under gentle shaking (60 cycles/min) at $37^{\circ} \mathrm{C}{ }^{35}$. The resulting suspension was filtered through a $200 \mu \mathrm{m}$ filter and adipocytes were washed once with DMEM-Ham's F12 to eliminate collagenase. Isolated mature adipocytes were diluted in DMEM-Ham's F12 supplemented with $3 \%$ bovine serum albumin for lipolysis assays and incubated with increasing final concentrations of isoprenalin (ISO, a non-selective beta-adrenergic agonist; $10^{-10}-10^{-3} \mathrm{~mol} / \mathrm{l}$ ) or human ANP (Bachem, Weil am Rhein, Germany) $\left(10^{-10}-10^{-4}\right.$ $\mathrm{mol} / \mathrm{l})$ in duplicate at a finale volume of $100 \mu \mathrm{l}$ for $3 \mathrm{~h}$ at $37^{\circ} \mathrm{C}$. Following incubation, $60 \mu \mathrm{l}$ cell free aliquots of the infranatant were collected for glycerol determination (lipolysis index) using the EnzyChromeTM Adipolysis assay kit (Gentaur, Eersel, The Netherlands). Glycerol release was expressed per $100 \mathrm{mg}$ of lipids, or per cell number, as previously described $33,36,37$. In addition, glycerol release at maximum effective concentration (compared to baseline) was determined. Lipid content was determined by a quantitative calorimetric method (ABX Pentra Triglycerides CP reagent, HORIBA Instruments LTD, The Netherlands). In brief, pooled cell suspension aliquots were mixed with $5 \%$ 
Nonidet P40 substitute (NP-40, Sigma Aaldrich, The Netherlands) and heated twice $\left(95^{\circ} \mathrm{C}\right)$ for 2 minutes and subsequently centrifuged for 2 minutes at $14.000 \mathrm{rpm}$ at $4^{\circ} \mathrm{C}$. Supernatant was collected and used for enzymatic TAG determination after incubation (20 minutes at $37^{\circ} \mathrm{C}$ ) using a Spectramax M2 microplate reader (Molecular devices, Berkshire, UK).

Gene expression experiments - Total AT RNA was isolated using the TRIzol ${ }^{\circledR}$ reagent (Invitrogen, Paisley, UK). Reverse transcription of $300 \mathrm{ng}$ of total RNA was performed using iScript CDNA synthesis kit (Bio-Rad) and real-time PCR was carried out on the MyiQ single color RT-PCR detection system (Bio-Rad). Reactions were performed in a total volume of $25 \mu \mathrm{L}$ containing $5.5 \mu \mathrm{L}$ complementary DNA (cDNA), $12.5 \mu \mathrm{L}$ SYBR green master mix (IQ SYBR Green Supermix) and gene specific primers for NPRA and NPRC (Biolegio, Nijmegen, The Netherlands). Gene expression was normalized using 185 ribosomal RNA. NPRA forward primer 5' GCAAAGGCCGAGTTATCTACAT and reverse primer 5'AAGAAAACGTAGTCCTCCCCA. NPRC forward primer AGACTACGCCTTCTTCA ACATTG and reverse primer 5' GCTTCAAAGTCGTGTTTGTCTCC.

Protein isolation - AT biopsies (100-300 mg) were ground to a fine powder under liquid nitrogen and homogenized in 200-600 $\mu \mathrm{L}$ radioimmunoprecipitation assay (RIPA) buffer supplemented with a protease/phosphatase inhibitor cocktail (Cell Signalling Technology Europe, Leiden, The Netherlands). Lysates were vortexed for $5 \mathrm{~min}$ at room temperature and centrifuged at $14.000 \mathrm{rpm}$ for 30 min at $10^{\circ} \mathrm{C}$. Infranatant was carefully transferred to new tubes. Protein concentrations were determined using the Pierce ${ }^{\circledR}$ BCA protein assay kit (Santa Cruz Biotechnology Inc., Heidelberg, Germany). Samples were stored at $-80^{\circ} \mathrm{C}$ prior to analysis.

Western blotting - $25 \mu \mathrm{g}$ protein was separated on Any kD SDS-PAGE gels (Bio-Rad Laboratories, Veenendaal, The Netherlands) and subsequently blotted semi-dry onto nitrocellulose membrane using Trans-Blot $®$ Turbo Transfer System (Bio-Rad Laboratories, Veenendaal, The Netherlands). Following transfer, membranes were blocked for $1 \mathrm{~h}$ in blocking buffer (Tris-buffered saline with $0.1 \%$ Tween 20 (TBS-T), 5\% nonfat dry milk). The membranes were incubated overnight at $4^{\circ} \mathrm{C}$ with primary antibodies diluted in blocking buffer. 
Next, membranes were incubated with the corresponding secondary antibodies for $1 \mathrm{~h}$ at room temperature. The primary antibodies used were anti-a-NPRC antibody (Sigma SAB2501867, 1:1000 dilution), anti-a-NPRA antibody (Abcam ab154266, 1:1000 dilution) and anti- $\beta$-actin antibody (Santa Cruz, \#sc-47778, $1: 1000$ dilution). Secondary antibodies were horse-radish peroxidaseconjugated-IgG anti-a-swine or anti-a-rabbit antibody (DAKO, Heverlee, Belgium). Antigen-antibody complexes were visualized by chemiluminescence using SuperSignalTM West Femto extended Duration Substrate (Life Technologies, Gent, Belgium). As positive control for NPRC, $1 \mu \mathrm{g}$ of a transient overexpression lysate of NPRC was loaded (Origene, \#LY424462). Visualization and analysis was performed using a Chemidoc XRS system (Bio-Rad Laboratories, CA, USA) and Quantity One software.

Statistical analysis - All data are presented as mean \pm SEM. Shapiro-Wilk tests indicated that data were not normally distributed. Therefore, group differences were analyzed using nonparametric Kruskal Wallis and Mann-Witney $U$ tests and depot differences within groups using Wilcoxon Signed rank tests. Statistical significance was set at $P<0.05$ (2-tailed). Statistical analyses were performed using SPSS 22.0 for Windows (SPSS Inc., Chicago, IL, USA). 


\section{Results}

\section{Subjects' characteristics}

Subjects' characteristics and use of glucose/lipid lowering and anti-hypertensive medication are displayed in Table 1. All groups were matched for age, and obese subjects differed from lean controls in several anthropometric and metabolic parameters, having higher body weight, and body fat, while their lipid profile (circulating lipids (NEFA and TAG)) and insulin sensitivity (as indicated by the higher HOMA-IR) were more disturbed. Obese subjects with type 2 diabetes showed higher $\mathrm{HbA} 1 \mathrm{C}$ and fasting glucose levels compared to lean subjects and obese subjects without type 2 diabetes. Fasting ANP levels were significantly higher in obese type 2 diabetic men $(29.4 \pm 1.7 \mathrm{pg} / \mathrm{ml})$ compared to lean men $(23.7 \pm 1.4 \mathrm{pg} / \mathrm{ml} ; \mathrm{P}<0.01)$, with intermediate values for obese men without type 2 diabetes. Both obese men with and without type 2 diabetes were on lipid $(n=8)$ and/or blood pressure $(n=17)$ lowering medication (including betablockers, diuretics, calcium channel blockers, angiotensin converting enzyme (ACE) inhibitors or a combination). Eight of the obese type 2 diabetic men were on blood glucose lowering medication. 
Lean
Obese without type 2

diabetes (18)
Obese with type 2

diabetes (11)

\begin{tabular}{lccc}
\hline Age, years & $51 \pm 1$ & $48 \pm 1$ & $51 \pm 2$ \\
BMI, kg/m² & $23.9 \pm 0.3$ & $37.9 \pm 1.0 *$ & $38.0 \pm 1.1^{*}$ \\
Waist circumference, cm & $91.3 \pm 1.6$ & $128.3 \pm 2.2 *$ & $126.1 \pm 1.9 *$ \\
Waist-to-hip ratio & $0.96 \pm 0.01$ & $1.08 \pm 0.01 *$ & $1.08 \pm 0.01 *$ \\
Fat mass, kg & $18.6 \pm 1.1$ & $45.8 \pm 2.7 *$ & $42.9 \pm 2.0 *$ \\
Body fat, \% & $23.8 \pm 1.2$ & $36.6 \pm 1.1 *$ & $36.7 \pm 1.0 *$ \\
Fasting glucose, mmol/l & $5.6 \pm 0.1$ & $5.7 \pm 0.2$ & $7.8 \pm 0.7 *, \dagger$ \\
Fasting insulin, mU/I & $9.3 \pm 1.1$ & $21.9 \pm 2.6 *$ & $21.3 \pm 3.2 *$ \\
Fasting NEFA, $\mu$ mol/l & $295 \pm 56$ & $759 \pm 88^{*}$ & $872 \pm 69 *$ \\
Fasting triglycerides, mmol/l & $1.1 \pm 0.1$ & $1.2 \pm 0.1 *$ & $1.2 \pm 0.2$ \\
Fasting ANP, pg/ml & $23.7 \pm 1.4$ & $25.3 \pm 1.6$ & $29.4 \pm 1.7 *$ \\
HbA $_{1 \mathrm{C},} \%$ & $5.3 \pm 0.1$ & $5.5 \pm 0.1$ & $7.3 \pm 0.3 *, \dagger$ \\
HbA $_{1 \mathrm{c},}$ mmol/mol & $35 \pm 1$ & $37 \pm 1$ & $56 \pm 3 *, \dagger$ \\
HOMA-IR $_{\text {Glucose lowering medication }}$ & $2.3 \pm 0.3$ & $5.7 \pm 0.8 *$ & $7.9 \pm 1.7 *$ \\
Lipid lowering medication & - & - & $8 / 11$ \\
Anti-hypertension medication & - & $4 / 18$ & $4 / 11$ \\
\hline
\end{tabular}

Values are means \pm SEM. BMI, body mass index; NEFA, non-esterified fatty acids; ANP, atrial natriuretic peptide; HOMA-IR, homeostatic model assessment of insulin resistance. *Significantly different from lean group $(P<0.01)$; $\dagger$ significantly different from obese group without type 2 diabetes $(P<0.01)$.

\section{Attenuated maximal ISO- and ANP-mediated lipolysis in mature adipocytes derived from the SCAT of obese men}

Glycerol release, a marker of complete TAG hydrolysis, was used to compare basal, ISO- and ANP-mediated adipocyte lipolysis in freshly isolated mature adipocytes of the SCAT and the VAT (Table 2). Basal lipolysis per cell was significantly higher in obese men with and without type 2 diabetes compared with lean men in both the SCAT and VAT. No differences between groups were observed in basal (non-stimulated) glycerol release when adjusted for the amount of lipids.

Incubation of freshly isolated mature adipocytes with ISO or ANP caused a concentration-dependent increase in glycerol release in both AT depots (Supplemental Figure 1). When expressing ISO-induced glycerol release per cell 
number, responses in both AT depots (Table 2) were significantly higher in both obese and obese diabetic men as compared to lean men. Maximal ISO-mediated lipolysis (expressed as fold-change over basal) was significantly reduced in the SCAT of obese (2.5-2.7-fold) compared to lean (3.8-fold) men $(P<0.05)$. This blunted maximal lipolytic response was most pronounced in adipocytes derived from obese subjects with type 2 diabetes and intermediate in obese subjects without type 2 diabetes. In VAT, no difference in maximal ISO-mediated lipolysis was observed between groups (Table 2). However, when comparing both depots, a higher maximal ISO-mediated lipolytic response was observed in adipocytes derived from VAT compared to SCAT of obese and obese type 2 diabetic subjects, which was not observed in lean controls (Table 2). In contrast to maximal response, $\beta$-adrenergic receptor sensitivity did not differ between groups or depots (data not shown).

Maximal ANP-mediated lipolysis expressed per cell number was not different between groups in the SCAT. However in the VAT, a higher maximal ANP response per cell was observed in adipocytes derived from obese compared to lean men, which was most obvious in obese subjects with type 2 diabetes as compared to lean men (4.3 vs. $1.4, \mathrm{P}<0.05)$. When corrected for basal lipolysis, maximal ANP-mediated lipid mobilization was significantly lower in the adipocytes derived from SCAT of obese type 2 diabetic subjects as compared to lean subjects ( 2.4 vs. 4.9, $\mathrm{P}<0.05$ ). In VAT, no differences in maximal ANP response were observed between groups when expressed as fold-change over basal lipolysis. When comparing both depots, lean subjects showed a significantly higher maximal ANP responsiveness in the adipocytes derived from the SCAT compared to the VAT (1.6-fold), while this depot difference was absent in both obese groups. In contrast to maximal lipolytic response, ANP-sensitivity did not differ between groups or depots (data not shown). 
Table 2 - Basal and maximal ISO- and ANP-mediated lipolytic response in SCAT and VAT adipocytes of lean, obese and

\section{obese diabetic men}

\begin{tabular}{|c|c|c|c|c|c|c|}
\hline & \multicolumn{3}{|c|}{ SCAT } & \multicolumn{3}{|c|}{ VAT } \\
\hline & Lean (13) & Obese (18) & Obese diabetics (11) & Lean (13) & Obese (18) & Obese diabetics (11) \\
\hline \multicolumn{7}{|l|}{ Basal } \\
\hline Glycerol ( $\mu \mathrm{mol} / \mathrm{l} / 3 \mathrm{~h}$ incubation) & $12.3 \pm 2.7 \dagger$ & $8.6 \pm 1.2+$ & $10.5 \pm 2.0$ & $20.0 \pm 5.3$ & $13.4 \pm 2.2$ & $13.6 \pm 1.7$ \\
\hline per $100 \mathrm{mg}$ lipids & $7.7 \pm 2.1$ & $6.5 \pm 0.8$ & $7.0 \pm 1.5$ & $8.4 \pm 2.3$ & $9.1 \pm 2.9$ & $6.4 \pm 0.9$ \\
\hline per $10^{6}$ cells & $0.44 \pm 0.09$ & $1.48 \pm 0.23^{* *}$ & $1.32 \pm 0.15^{* *}$ & $0.67 \pm 0.20$ & $1.19 \pm 0.16^{* *}$ & $1.81 \pm 0.52 *$ \\
\hline \multicolumn{7}{|l|}{ Isoprenalin $\left(10^{-6} \mathrm{~mol} / \mathrm{I}\right)$} \\
\hline per $100 \mathrm{mg}$ lipids & $24.0 \pm 5.1$ & $15.8 \pm 1.6$ & $20.8 \pm 8.1$ & $45.9 \pm 19.0$ & $48.5 \pm 18.6$ & $22.7 \pm 4.4$ \\
\hline per $10^{6}$ cells & $1.62 \pm 0.31$ & $3.65 \pm 0.54 * *$ & $3.94 \pm 1.51$ & $2.42 \pm 0.73$ & $4.99 \pm 0.78^{* *}$ & $6.06 \pm 1.55$ \\
\hline fold compared to basal & $3.78 \pm 0.50$ & $2.72 \pm 0.26 \dagger$ & $2.54 \pm 0.78^{*}$ & $4.69 \pm 0.79$ & $4.44 \pm 0.41$ & $3.72 \pm 0.72$ \\
\hline \multicolumn{7}{|l|}{ ANP $\left(10^{-4} \mathrm{~mol} / \mathrm{I}\right)$} \\
\hline per $100 \mathrm{mg}$ lipids & $26.0 \pm 5.1$ & $17.2 \pm 2.2$ & $18.5 \pm 7.3$ & $23.1 \pm 7.2$ & $21.7 \pm 7.1$ & $15.4 \pm 2.8$ \\
\hline per $10^{6}$ cells & $1.86 \pm 0.31$ & $4.08 \pm 0.64$ & $3.70 \pm 1.54$ & $1.45 \pm 0.33$ & $2.52 \pm 0.49$ & $4.30 \pm 1.16^{*}$ \\
\hline fold compared to basal & $4.97 \pm 0.88 \dagger$ & $3.09 \pm 0.46$ & $2.41 \pm 0.82 *$ & $2.98 \pm 0.50$ & $2.23 \pm 0.31$ & $2.60 \pm 0.51$ \\
\hline
\end{tabular}




\section{Reduced NPRA and increased NPRC expression in the adipose tissue of obese subjects}

In line with the attenuated maximal ANP response in adipocytes derived from SCAT of obese men, NPRA mRNA expression was lower in adipose tissue biopsies derived from obese compared to lean SCAT (0.63 vs. $1.00, P<0.01$, Figure 1A). In VAT, NPRA mRNA expression was comparable between groups (Figure 1A). In contrast to NPRA, the NPRC transcript was more abundant in both SCAT and VAT biopsies of obese (1.83 and 1.17, respectively) and obese type 2 diabetics ( 1.78 and 1.34 , respectively) compared to lean men $(1.00$ ( $P<$ 0.05 ) and $0.54(P<0.01)$, respectively), resulting in a decreased NPRA:NPRC ratio (i.e. NPRR) in obese subjects (Figure $1 \mathrm{~A}$ ). When comparing both depots, NPRC mRNA expression was lower in the VAT compared to the SCAT of lean and obese men $(P<0.05)$, while NPRA expression was higher in the VAT compared to the SCAT of obese diabetic subjects $(P<0.05$, Figure $1 A)$.

A

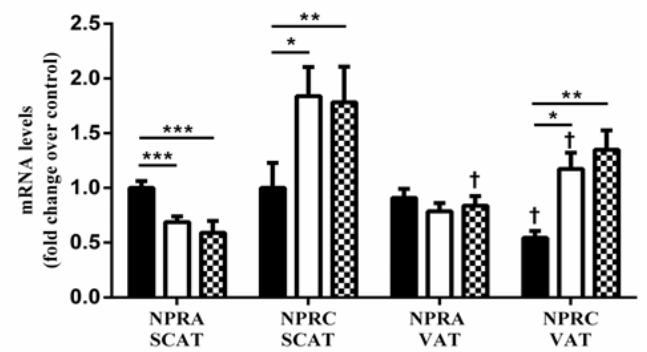

B

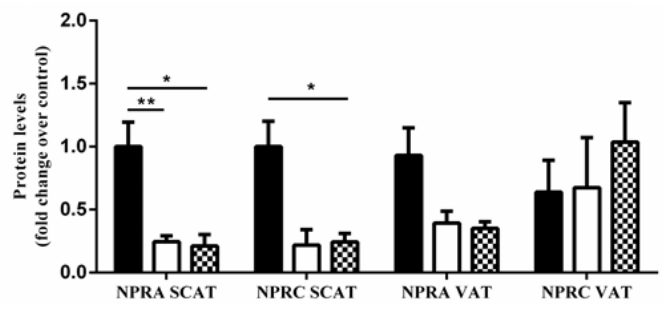

C

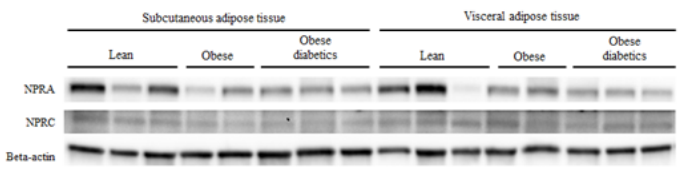

Figure 1 - mRNA and protein expression of NPRA and NPRC in SCAT and VAT of lean, obese and obese diabetic men. (A) mRNA expression of NPRA and NPRC were determined in paired AT samples from 30 obese and 14 lean subjects. (B) Protein expression data of NPRA was derived from paired AT samples of lean $(n=7)$, obese $(n=7)$ and obese diabetic men $(n=6)$. Protein expression data of NPRC was derived from paired AT samples of lean $(n=6)$, obese $(n=4)$ and obese diabetic men $(n=6)$. (C) Representative western blot for NPRA and NPRC in SCAT and VAT of lean $(n=3)$ and obese men with $(n=3)$ and without type 2 diabetes $(n=2)$. Black bars indicate lean, white bars indicate obese and white squared bars indicate obese diabetic men. $* P<0.05$; ** $P<0.01$; *** $P<0.001 ;+P<0.05$ between depots of same subject group. 
Western Blot analysis (Figure $1 \mathrm{~B}$ and $\mathrm{C}$ ) revealed that both receptors were downregulated at the protein level in SCAT biopsies of obese subjects. Both obese $(4.1$-fold for NPRA $(P<0.01)$ and 4.5 -fold for NPRC $(P=0.05)$ ) and obese type 2 diabetic men $(4.7$-fold for NPRA $(P<0.05)$ and 4.1 -fold for NPRC $(P<0.05)$ ) showed lower NP receptor levels compared with lean men. No difference in NP receptor protein content was found in the VAT between groups (Figure 1B). When comparing between depots, a trend towards a higher NPRC protein expression was found in the VAT of obese subjects with type 2 diabetes compared to the SCAT (4.2-fold, $\mathrm{P}=0.07)$. Additionally, NPRA protein expression tended to be lower in SCAT compared to VAT in non-diabetic obese subjects ( $P$ $=0.07$ ) (Figure 1B). 


\section{Discussion}

In the present study, we investigated for the first time both catecholamine- and ANP-mediated lipolysis in visceral and subcutaneous adipocytes derived from lean compared to obese men with and without type 2 diabetes. Our data clearly demonstrate an attenuated maximal responsiveness to the two major lipolytic hormones, ANP and catecholamines (i.e. ISO) in the SCAT of obese men (independent of diabetes status). The impaired ANP-mediated lipolysis was accompanied by a differential NP receptor expression pattern. Of interest, in lean men, ANP-mediated lipid mobilization was higher in SCAT than VAT but this depot difference disappeared in both obese groups.

In the current study basal and ANP-mediated glycerol release, expressed per cell, were increased in obese hypertrophic subcutaneous adipocytes, although this did not reach statistical significance. These observations are in agreement with recent ex vivo data from Rydén et al. indicating comparable ANP-mediated lipolysis in obese compared to non-obese women when expressed per fat cell number 22. Maximal ISO-mediated glycerol release from subcutaneous adipocytes was higher in obese when expressed per cell and lower when expressed per cell mass (i.e. lipid content), a consequence of the increased average size and mass of adipocytes in obese men. In line, ex vivo basal and maximal stimulated (ANP- and ISO-mediated) lipolysis has been shown to be higher in large versus small adipocytes within the same depot of healthy lean and obese men and women 38,39. However, when maximal ANP- and ISOinduced glycerol release were expressed as a ratio of basal release, we observed an attenuated maximal lipolytic responsiveness to both ANP and ISO in subcutaneous adipocytes of obese men (independent of diabetes status). Previously, both a decreased maximal catecholamine-induced lipolytic response and attenuated beta-adrenergic receptor sensitivity have been observed in SCAT of obese subjects ${ }^{12,13}$. With respect to ANP, previous data from Rydén et al. also reported only an attenuated maximal ANP response and no difference in ANP sensitivity between obese and non-obese women ${ }^{22}$. Table 3 gives a detailed overview of the in vivo and in vitro human studies on ANP-mediated lipolysis in adipose tissue performed so far. Moreover, we observed a more pronounced decrease in maximal ANP-mediated lipolysis in obese type 2 diabetic men 
compared to obese insulin resistant men, illustrating that this impaired ANP response in the SCAT is related to severity of metabolic abnormalities. Together these data indicate the presence of a state of impaired lipid turnover, which together with the attenuated maximal catecholamine- and ANP-mediated lipolytic response in SCAT may contribute to an impaired adipose tissue lipid metabolism in obesity and the subsequent development of insulin resistance.

Of interest we observed a reciprocal regulation of ANP- and catecholaminemediated lipolysis in subcutaneous and visceral adipocytes from lean men, which was not present in obese men. The higher ISO- and lower ANP-induced lipolysis in visceral compared to subcutaneous adipocytes of lean men, might be partly explained by differences in receptor density or function or post-receptor signaling ${ }^{14}$. In line with our observations in obese men, Dicker et al. investigated potential depot-specific differences in ANP-mediated lipolysis in middle-aged, morbidly obese women and did not find any difference between visceral and subcutaneous differentiated preadipocytes (Table 3$)^{23}$. Consistent with these data our data showing no depot differences in ANP-mediated lipolysis between depots in the obese groups as compared to a reduced ANP-mediated lipolysis in VAT as compared to SCAT in the lean group, support the presence of an ANP-resistant phenotype at the level of SCAT in obese subjects.

Since the net lipolytic effect of ANP is determined by both NPRA and scavenging NPRC ${ }^{36}$, we investigated the expression level of both receptors to get some more insight on the underlying mechanism responsible for the observed attenuated maximal ANP responsiveness in the SCAT of obese men. In line with our ex vivo data, we found a decreased expression of NPRA in SCAT, both at mRNA (as shown previously ${ }^{24}$ ) and protein level, while VAT showed comparable levels for NPRA (mRNA and protein level) between groups. Regarding ANP scavenging, NPRC mRNA levels were higher in obese compared to lean men in both AT depots. However, this did not translate into higher NPRC protein levels, which might be a compensatory mechanism for the observed higher circulating ANP levels in obese men. Due to the absence of a catalytic GC domain, the ANP scavenging NPRC receptor has a very minor role in ANP-mediated lipolysis. Its main function is to facilitate scavenging of its ligands, internalization of ligandreceptor complexes and recycling of NPRC, together with degradation of its 
ligands 26. However, the neutral endopeptidase neprilysin, an enzyme responsible for processing of NPs, was shown to have a minor function in human adipocytes and therefore, in contrast to NPRA, NPRC might not play a major role in the regulation of ANP-mediated lipolysis in human adipose tissue ${ }^{33}$. In addition, comparable NPRA and NPRC levels were observed between both adipose tissue depots in lean as well as in obese men. Furthermore, as maximal lipolytic response is determined by both receptor and post-receptor signaling, also more downstream impairments might have contributed to the observed blunted lipolytic response in the SCAT of obese men and the depot-specific regulation of ANP- and ISO-mediated lipolysis as observed in lean men. In line, others and we have shown previously that expression and activity of the lipid droplet associated lipases ATGL and HSL is decreased in SCAT of obese insulinresistant men $11,40,41$, possibly contributing to the observed attenuated ANP- and ISO-mediated lipolysis in subcutaneous adipocytes of obese men. Another important downstream second messenger is cGMP, which is formed by the soluble guanylyl cyclase-pathway or via membrane-bound guanylyl cyclase upon binding of NP to its NPRA receptor ${ }^{42}$. Evidence about the importance of the NPRA-linked cGMP/PKG pathway in browning of white AT and increasing energy expenditure is accumulating ${ }^{43}$. In combination with the results regarding an imperative role of NP in AT lipolysis, these data suggest that the NP system might be a promising target for anti-obesity drug development ${ }^{28}$. Unfortunately, clinical trials investigating the effects of (non)pharmacological stimulation of NP on obesity and related cardiometabolic disease are currently lacking ${ }^{44}$.

Together, our data suggest a decreased number of functional NPRA receptors and decreased ANP scavenging in the SCAT of obese insulin resistant men, which is in line with our observed attenuated maximal ANP responsiveness in the SCAT and increased circulating ANP levels in obese men. However, this contrasts previous data showing lower circulating ANP levels in obese men ${ }^{45-47}$. This discrepancy results probably from a worse cardiovascular health in our obese group, indicated by the elevated (systolic) blood pressure and use of antihypertensive drugs by more than $70 \%$ of the obese men as compared to 0 $38.5 \%$ in other studies ${ }^{45,46}$. 
Table 3 - Summary of studies on ANP-mediated lipolysis

\begin{tabular}{|c|c|c|c|c|}
\hline Methodology & Participants & Adipose tissue & Effect on lipolysis & Study \\
\hline \multicolumn{5}{|l|}{ In vitro studies } \\
\hline $\begin{array}{r}\text { differentiated preadipocytes stimulated with } \\
\text { ANP }\left(10^{-11}-10^{-6} \mathrm{~mol} / \mathrm{L}\right)\end{array}$ & $\begin{array}{l}10 \text { obese women (BMI } \\
\left.43.8 \pm 3.5 \mathrm{~kg} / \mathrm{m}^{2}\right)\end{array}$ & SCAT vs. VAT & $\begin{array}{l}\text { ANP-mediated lipolysis did not differ } \\
\text { between adipose tissue depots }\end{array}$ & $\begin{array}{l}\text { Dicker et al. } \\
(2008)^{23}\end{array}$ \\
\hline \multicolumn{5}{|l|}{ Ex vivo isolated mature adipocytes } \\
\hline stimulated with hANP $\left(10^{-10}-10^{-6} \mathrm{~mol} / \mathrm{L}\right)$ & $\begin{array}{l}7 \text { healthy lean (BMI } 22.2 \pm 0.7 \\
\left.\mathrm{~kg} / \mathrm{m}^{2}\right) \text { and } 7 \text { healthy obese } \\
\left(\mathrm{BMI} 31.5 \pm 1.4 \mathrm{~kg} / \mathrm{m}^{2}\right)\end{array}$ & SCAT & $\begin{array}{l}\text { Comparable hANP responsiveness } \\
\text { and sensitivity between lean and } \\
\text { obese }\end{array}$ & $\begin{array}{l}\text { Galitzky et al. } \\
\qquad(2001)^{19}\end{array}$ \\
\hline $\begin{array}{l}\text { stimulated with ANP }\left(10^{-11}-10^{-6} \mathrm{~mol} / \mathrm{L}\right) \text { with or } \\
\text { without prior ANP }\left(10^{-6} \mathrm{~mol} / \mathrm{L}\right) \text { treatment }(1 \mathrm{~h})\end{array}$ & $\begin{array}{l}6 \text { healthy lean/moderately } \\
\text { overweight (BMI } 24.4 \pm 1.3 \\
\mathrm{~kg} / \mathrm{m}^{2} \text { ) women }\end{array}$ & SCAT & $\begin{array}{l}\text { In vitro desensitization of ANP- } \\
\text { induced lipolysis following prior ANP } \\
\text { treatment }\end{array}$ & $\begin{array}{l}\text { Moro et al. } \\
(2005)^{52}\end{array}$ \\
\hline $\begin{array}{l}\text { stimulated with increasing ANP concentrations } \\
\qquad\left(10^{-17}-10^{-7} \mathrm{~mol} / \mathrm{L}\right)\end{array}$ & $\begin{array}{l}35 \text { non-obese (BMI } 24 \pm 2 \\
\mathrm{~kg} / \mathrm{m}^{2} \text { ) and } 87 \text { obese (BMI } \\
\left.42 \pm 6 \mathrm{~kg} / \mathrm{m}^{2}\right) \text { women }\end{array}$ & SCAT & $\begin{array}{l}\text { Lower ANP responsiveness in SCAT } \\
\text { of obese women but no difference } \\
\text { in ANP sensitivity }\end{array}$ & $\begin{array}{l}\text { Rydén et al. } \\
\quad(2015)^{22}\end{array}$ \\
\hline \multicolumn{5}{|l|}{ In vivo studies } \\
\hline \multirow[t]{3}{*}{$\begin{array}{r}\text { in situ microdialysis infusion }(60 \mathrm{~min}) \text { with } \\
\text { hANP }\left(10^{-6} \mathrm{~mol} / \mathrm{L}\right)\end{array}$} & $\begin{array}{l}7 \text { healthy lean (BMI } 23.0 \pm 1.6 \\
\mathrm{~kg} / \mathrm{m}^{2} \text { ) men }\end{array}$ & SCAT & $\begin{array}{l}\text { SCAT lipolysis increased } \\
\text { significantly }(\sim 250 \%) \text { compared to } \\
\text { baseline }\end{array}$ & $\begin{array}{l}\text { Sengenès et al. } \\
\qquad(2000)^{18}\end{array}$ \\
\hline & $\begin{array}{l}7 \text { healthy lean (BMI } 22.2 \pm 0.7 \\
\left.\mathrm{~kg} / \mathrm{m}^{2}\right) \text { and } 7 \text { healthy obese } \\
\left(\mathrm{BMI} 31.5 \pm 1.4 \mathrm{~kg} / \mathrm{m}^{2}\right) \text { men }\end{array}$ & SCAT & $\begin{array}{l}\text { SCAT lipolysis increased } \\
\text { significantly }(\sim 200 \%) \text { compared to } \\
\text { baseline, comparable between } \\
\text { groups }\end{array}$ & $\begin{array}{l}\text { Galitzky et al. } \\
\qquad(2001)^{19}\end{array}$ \\
\hline & $\begin{array}{l}7 \text { healthy lean (BMI } 23.0 \pm 1.6 \\
\mathrm{~kg} / \mathrm{m}^{2} \text { ) and } 9 \text { healthy } \\
\text { overweight (BMI } 28.0 \pm 0.3 \\
\mathrm{~kg} / \mathrm{m}^{2} \text { ) men }\end{array}$ & SCAT & $\begin{array}{l}26 \% \text { reduction in ANP-induced } \\
\text { lipolysis in SCAT of overweight men } \\
\text { as compared to the lean }\end{array}$ & $\begin{array}{l}\text { Rydén et al. } \\
\quad(2015)^{22}\end{array}$ \\
\hline $\begin{array}{r}\text { intravenous infusion of hANP }\left(2.10^{-12}\right. \\
4.10^{-12} \text { and } 8.10^{-12} \mathrm{~mol} / \mathrm{kg} \cdot \mathrm{min} \text { over } 45 \mathrm{~min} \\
\text { each })\end{array}$ & $\begin{array}{l}14 \text { healthy lean (BMI } 24.2 \pm 0.4 \\
\mathrm{~kg} / \mathrm{m}^{2} \text { ) men }\end{array}$ & SCAT & $\begin{array}{l}\text { Significant increase in SCAT } \\
\text { lipolysis with increasing serum ANP } \\
\text { concentrations }\left(r^{2}=0.84\right)\end{array}$ & $\begin{array}{l}\text { Birkenfeld et al. } \\
\qquad(2005)^{20}\end{array}$ \\
\hline
\end{tabular}

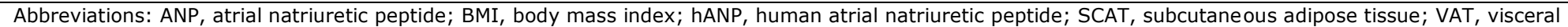
adipose tissue. 
Since it is evident that medication used in the treatment of cardiovascular disorders can modulate ANP levels and tissue responses this should be taken into account when interpreting the present data on ANP and catecholamine responsiveness in human AT of metabolically compromised donors ${ }^{48}$. In line with previous data from Pivovarova et al., showing an up-regulation of NPRC mRNA expression by insulin in the obese insulin-resistant state in VAT and SCAT ${ }^{24}$, we observed a higher NPRC mRNA expression in the SCAT and VAT in obese and obese diabetic subjects and an increased NPRC protein expression in VAT of obese diabetic subjects. Together, these data show that both adiposity and insulin sensitivity may influence ANP responsiveness in SCAT, which needs to be investigated in more detail in future studies aimed to improve insulin sensitivity (e.g. physical training or weight loss). Of interest, exercise training, which improves insulin sensitivity, is accompanied by an increased ANP-induced lipolysis in the SCAT, at least in young overweight men ${ }^{49}$. In addition, it was shown recently that substantial weight loss following bariatric surgery normalized ANP-induced lipolysis in the SCAT of obese women ${ }^{22}$. Together, these data suggest that ANP-induced lipolysis in the SCAT is reversible attenuated in human obesity and the use of exercise to optimize intervention effects related to fat mass loss and insulin sensitivity in the obese seems a promising strategy. 


\section{Conclusion}

In conclusion, our data show that maximal ANP- and catecholamine-induced lipolysis ${ }^{13,14}$ in the SCAT are attenuated in obese diabetic men as compared to lean controls with intermediate values for obese insulin-resistant men. This blunted ANP-mediated lipolytic response was accompanied by a decreased NPRA receptor expression, which was partly correlated with measures of adiposity and insulin sensitivity. These data suggest that improving maximal ANP responsiveness in the SCAT might be a potential novel strategy to improve lipid mobilization in obesity, which may possibly result in a more beneficial cardiometabolic profile. However, it remains to be determined if these improvements could be equally obtainable in different cardiometabolic phenotypes within the obese population ${ }^{50}$. Lifestyle as well as pharmacological interventions might be beneficial in this regard ${ }^{51}$.

\section{Acknowledgements}

We thank all abdominal surgeons from the Jessa Hospital (Hasselt, Belgium) and Hospital East-Limburg (Genk, Belgium) and Sandra Latet (Obesity Center Hasselt) for their contributions to subject recruitment. We thank University Biobank Limburg (UBiLim, Jessa Hospital, Belgium) for their technical support in sample handling and storage. 


\section{References}

1. Lafontan M. Adipose tissue and adipocyte dysregulation. Diabetes \& Metabolism 2014; 40:16-28

2. Virtue $S$, Vidal-Puig A. Adipose tissue expandability, lipotoxicity and the metabolic syndrome - an allostatic perspective. Biochim Biophys Acta 2010; 1801:338-349

3. Karastergiou K, Fried SK. Multiple adipose depots increase cardiovascular risk via local and systemic effects. Curr Atheroscler Rep 2013; 15:361

4. Whitlock G, Lewington S, Sherliker P, Clarke R, Emberson J, Halsey J et al. Body-mass index and cause-specific mortality in 900,000 adults: collaborative analyses of 57 prospective studies. Lancet 2009; 373:1083-1096

5. Frayn KN. Adipose tissue as a buffer for daily lipid flux. Diabetologia 2002; 45:12011210

6. Frühbeck G, Méndez-Giménez L, Fernandéz-Formoso JA, Fernandéz S, Rodriguez A. Regulation of adipocyte lipolysis. Nutr Research Rev 2014; 27:63-93

7. Langin D, Dicker A, Tavernier G, Hoffstedt J, Mairal A, Rydén M et al. Adipocyte lipases and defect of lipolysis in human obesity. Diabetes 2005; 54:3190-3197

8. Large V, Arner P. Regulation of lipolysis in humans. Pathophysiological modulation in obesity, diabetes, and hyperlipidaemia. Diabetes Metab 1998; 24:409-418

9. McQuaid SE, Hodson L, Neville MJ, Dennis AL, Cheeseman J, Humphreys SM et al. Downregulation of adipose tissue fatty acid trafficking in obesity - a driver for ectopic fat deposition. Diabetes 2011; 60:47-55

10. Jocken JWE, Goossens GH, van Hees AM, Frayn KN, van Baak M, Stegen J et al. Effect of beta-adrenergic stimulation on whole-body and abdominal subcutaneous adipose tissue lipolysis in lean and obese men. Diabetologia 2008; 51:320-327

11. Rydén M, Jocken JWE, van Harmelen V, Dicker A, Hoffstedt J, Wiren $M$ et al. Comparative studies of the role of hormone-sensitive lipase and adipose triglyceride lipase in human fat cell lipolysis. Am J Physiol Endocrinol Metab 2007; 292:E18471855

12. Lonnqvist $F$, Thome A, Nilsell K, Hoffstedt J, Arner P. A pathogenic role of visceral fat beta 3-adrenoceptors in obesity. J Clin Invest 1995; 95:1109-1116

13. Hoffstedt J, Arner P, Hellers G, Lönnqvist F. Variation in adrenergic regulation of lipolysis between omental and subcutaneous adipocytes from obese and non-obese men. J Lipid Res 1997; 38:795-804

14. Arner P. Differences in lipolysis between human subcutaneous and omental adipose tissues. Ann Med 1995;27:435-438

15. Mauriege P, Depres JP, Prud'homme D, Pouliot MC, Marcotte M, Tremblay A et al. Regional variation in adipose tissue lipolysis in lean and obese men. J Lipid Res 1991; 32:1625-1633

16. Dahlman I, Arner P. Obesity and polymorphisms in genes regulating human adipose tissue. Int J Obes 2007; 31:1629-1641

17. Terra SG, McGorray SP, Wu R, McNamara DM, Cavallari LH, Walker JR et al. Association between b-adrenergic receptor polymorphisms and their G-proteincoupled receptors with body mass index and obesity in women: a report from the NHLBI-sponsored WISE study. Int J Obes 2005; 29:746-754

18. Sengenes C, Berlan M, De Glisezinski I, Lafontan M, Galitzky J. Natriuretic peptides: a new lipolytic pathway in human adipocytes. FASEB J 2000;14:1345-1351

19. Galitzky J, Sengenes C, Thalamas C, Marques MA, Senard JM, Lafontan M et al. The lipid-mobilizing effect of atrial natriuretic peptide is unrelated to sympathetic nervous system activation or obesity in young obese men. J Lipid Res 2001; 42:536-544 
20. Birkenfeld AL, Boschmann M, Moro C, Adams F, Heusser K, Franke G et al. Lipid mobilization with physiological atrial natriuretic peptide concentrations in humans. J Clin Endocrinol Metab 2005; 90:3622-3628

21. Birkenfeld AL, Boschmann M, Moro C, Adams F, Heusser K, Tank J et al. Betaadrenergic and atrial natriuretic peptide interactions on human cardiovascular and metabolic regulation. J Clin Endocrinol Metab 2006; 91:5069-5075

22. Rydén $M$, Backdahl J, Petrus $P$, Thorell $A$, Gao $H$, Coue $M$ et al. Impaired atrial natriuretic peptide-mediated lipolysis in obesity. Int J Obes 2015; Epub ahead of print. doi: $10.1038 /$ ijo.2015.222

23. Dicker A, Aström G, Wahlen K, Hoffstedt J, Naslund E, Wiren M et al. Primary Differences in Lipolysis between Human Omental and Subcutaneous Adipose Tissue Observed Using In Vitro Differentiated Adipocytes. Horm Metab Res 2009; 41:350-355

24. Pivovarova O, Gögebakan O, Klöting N, Sparwasser A, Weickert MO, Haddad I et al. Insulin up-regulates natriuretic peptide clearance receptor expression in the subcutaneous fat depot in obese subjects: a missing link between CVD risk and obesity? J Clin Endocrinol Metab 2012; 97:E731-E739

25. Sengenes C, Bouloumié A, Hauner $H$, Berlan $M$, Busse $R$, Lafontan $M$ et al. Involvement of a cGMP-dependent pathway in the natriuretic peptide-mediated hormone-sensitive lipase phosphorylation in human adipocytes. J Biol Chem 2003; 278:48617-48626

26. Matsukawa N, Grzesik WJ, Takahashi N, Pandey KN, Pang S, Yamauchi M et al. The natriuretic peptide clearance receptor locally modulates the physiological effects of the natriuretic peptide system. Proc Natl Acad Sci USA 1999; 96:7403-7406

27. Potter LR, Abbey-Hosch S, Dickey DM. Natriuretic peptides, their receptors, and cyclic guanosine monophosphate-dependent signaling functions. Endocrine Reviews 2006; 27:47-72

28. Bordicchia M, Liu D, Amri EZ, Ailhaud G, Dessi-Fulgheri P, Zhang C et al. Cardiac natriuretic peptides act via p38 MAPK to induce the brown fat thermogenic program in mouse and human adipocytes. J Clin Invest 2012; 122:1022-1036

29. Sarzani R, Paci VM, Zingaretti CM, Pierleoni C, Cinti S, Cola G et al. Fasting inhibits natriuretic peptides clearance receptor expression in rat adipose tissue. J Hypertens 1995; $13: 1241-1246$

30. Nakatsuji $H$, Maeda N, Hibuse $T$, Hiuge A, Hirata A, Kuroda $Y$ et al. Reciprocal regulation of natriuretic peptide receptors by insulin in adipose cells. Biochem Biophys Res Comm 2010; 392:100-105

31. Dessi-Fulgheri P, Sarzani R, Tamburrini P, Moraca A, Espinosa E, Cola G et al. Plasma atrial natriuretic peptide and natriuretic peptide receptor gene expression in adipose tissue of normotensive and hypertensive obese patients. J Hypertens 1997;15:16951699

32. Kovacova Z, Tharp WG, Liu D, Wei W, Xie H, Collins S et al. Adipose tissue natriuretic peptide receptor expression is related to insulin sensitivity in obesity and diabetes. Obesity 2016; Epub ahead of print. doi:10.1002/oby.21418

33. Moro C, Klimcakova E, Lafontan M, Berlan M, Galitsky J. Phosphodieterase-5A and neutral endopeptidase activities in human adipocytes do not control atrial natriuretic peptide-mediated lipolysis. British J Pharmacol 2007; 152:1102-1110

34. Matthews DR, Hosker JP, Rudenski AS, Naylor BA, Treacher DF, Turner RC. Homeostasis model assessment: insulin resistance and beta-cell function from fasting plasma glucose and insulin concentration in man. Diabetologia $1985 ; 28: 412-419$

35. Rodbell M. Metabolism of isolated fat cells - effects of hormones on glucose metabolism and lipolysis. J Biol Chem 1964; 239: 375-380 
36. Moro C, Galitzky J, Sengenes C, Crampes F, Lafontan M, Berlan M. Functional and pharmacological characterization of the natriuretic peptide-dependent lipolytic pathway in human fat cells. J Pharmacol Exp Ther 2004; 308: 984-992

37. Moro C, Pasarica M, Elkind-Hirsch K, Redman LM. Aerobic exercise training improves atrial natriuretic peptide and catecholamine-mediated lipolysis in obese women with polycystic ovary syndrome. J Clin Endocrinol Metab 2009; 94:2579-2586

38. Yu J, Yu HC, Kim KA, Kwon KB, Park JW, Kim SZ et al. Differences in the amount of lipolysis induced by atrial natriuretic peptide in small and large adipocytes. J Pept Sci 2008; 14:972-977

39. Laurencikiene J, Skurk T, Kulyte A, Heden P, Astrom G, Sjolin E et al. Regulation of lipolysis in small and large fat cells of the same subject. J Clin Endocrinol Metab 2011; 96:E2045-2049

40. Jocken JW, Langin D, Smit E, Saris WH, Valle C, Hul GB et al. Adipose triglyceride lipase and hormone-sensitive lipase protein expression is decreased in the obese insulin-resistant state. J Clin Endocrinol Metab 2007; 92:2292-2299

41. Langin D, Dicker A, Tavernier G, Hoffstedt J, Mairal A, Rydén M et al. Adipocyte lipases and defect of lipolysis in human obesity. Diabetes 2005; 54:3190-3197

42. Derbyshire ER; Marletta MA. Structure and regulation of soluble guanylate cyclase. Annu Rev Biochem 2012; 81:533-559

43. Leiss V, Illison J, Domes K, Hofmann F, Lukowski R. Expression of cGMP-dependent protein kinase type I in mature white adipocytes. Biochem Biophys Res Commun $2014 ; 452: 151-156$

44. Hofmann LS, Chen HH. Cgmp: transition from bench to bedside: a report of the 6th international conference on cgmp generators, effectors and therapeutic implications. Naunyn Schmiedebergs Arch Pharmacol 2014; 387:707-718

45. Olsen MH, Hansen TW, Christensen MK, Gustafsson F, Rasmussen S, Wachtell K et al. $\mathrm{N}$-terminal pro brain natriuretic peptide is inversely related to metabolic cardiovascular risk factors and the metabolic syndrome. Hypertension 2005; 46:660666

46. Magnusson M, Jujic A, Hedblad B, Engstrom G, Persson M, Struck J et al. Low plasma level of atrial natriuretic peptide predicts development of diabetes: the prospective Malmo Diet and Cancer study. J Clin Endocrinol Metab 2012; 97:638-645

47. Khan AM, Cheng S, Magnusson M, Larson MG, Newton-Cheh C, McCabe EL et al. Cardiac natriuretic peptides, obesity, and insulin resistance: evidence from two community-based studies. J Clin Endocrinol Metab 2011; 96:3242-3249

48. Troughton RW, Richards AM, Yandle TG, Frampton CM, Nicholls MG. The effects of medications on circulating levels on circulating levels of cardiac natriuretic peptides. Ann Med 2007; 39:242-260

49. Moro C, Pillard F, De Glisezinski I, Harant I, Riviere D, Stich V et al. Training enhances ANP lipid-mobilizing action in adipose tissue of overweight men. Med Sci Sports Exerc 2005; 37:1126-1132

50. Blüher M. Are metabolically healthy obese individuals really healthy? Eur J Endocrinol 2014; 171:R209-2019

51. McMurray J], Packer M, Desai AS, Gong J, Lefkowitz MP, Rizkala AR et al. Angiotensin-neprilysin inhibition versus enalapril in heart failure. N Engl J Med 2014; 371:993-1004

52. Moro C, Polak J, Richterova B, Sengenès C, Pelikanova T, Galitzky J et al. Differential regulation of atrial natriuretic peptide- and adrenergic receptoro-dependent lipolytic pathways in human adipose tissue. Metabolism 2005; 54:122-131 


\section{Supplemental data}
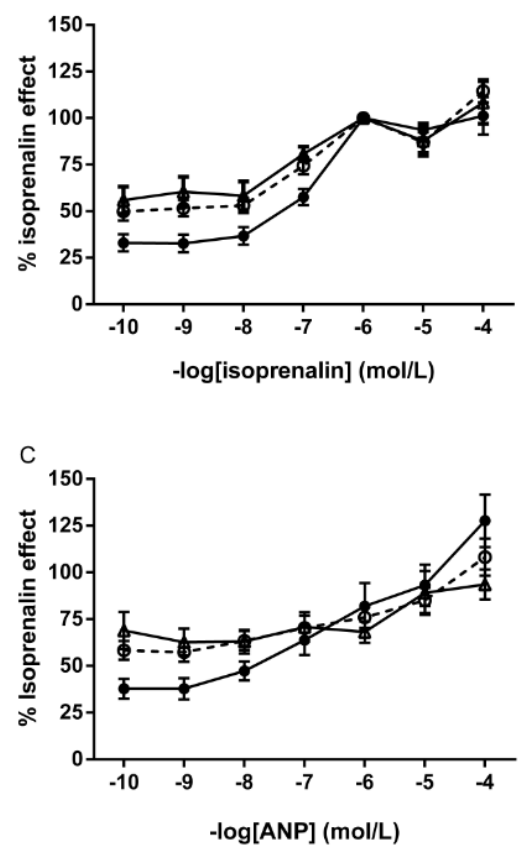

B

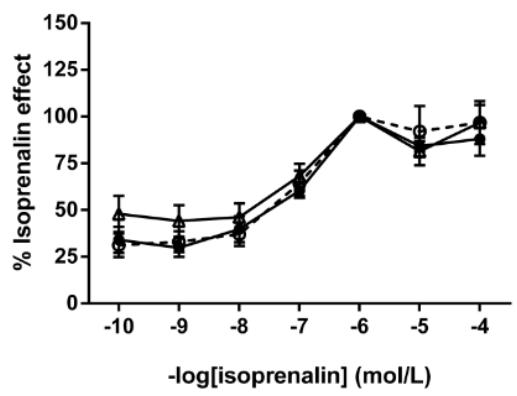

D

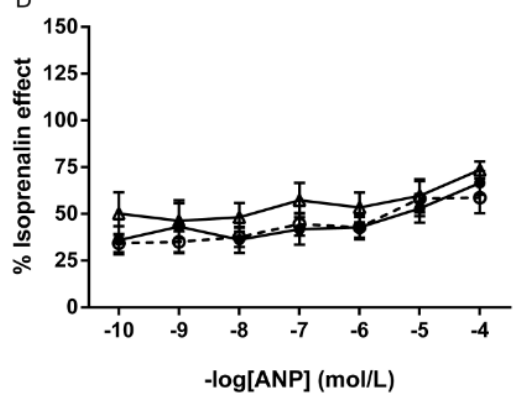

Figure S1 - Dose-response curves for ISO- or ANP-mediated lipolytic response in human mature adipocytes derived from the SCAT and VAT of lean, obese and obese diabetic male donors. Lipolysis induced by nonselective beta-adrenergic agonist isoprenalin (ISO) (A,B) or ANP $(\mathbf{C}, \mathbf{D})$ in subcutaneous $(\mathbf{A}, \mathbf{C})$ and visceral $(\mathbf{B}, \mathbf{D})$ isolated mature adipocytes of lean ( $n=13$, black circles), obese $(n=17$, dotted line, white circles) and obese diabetic $(n=11$, white triangles). Lipolysis is expressed in percent maximum effect of isoprenalin, following incubation with increasing concentrations ISO $\left(10^{-10}-10^{-4} \mathrm{~mol} / \mathrm{I}\right)$ or ANP $\left(10^{-10}-10^{-4} \mathrm{~mol} / \mathrm{l}\right)$ for each adipose tissue depot respectively.

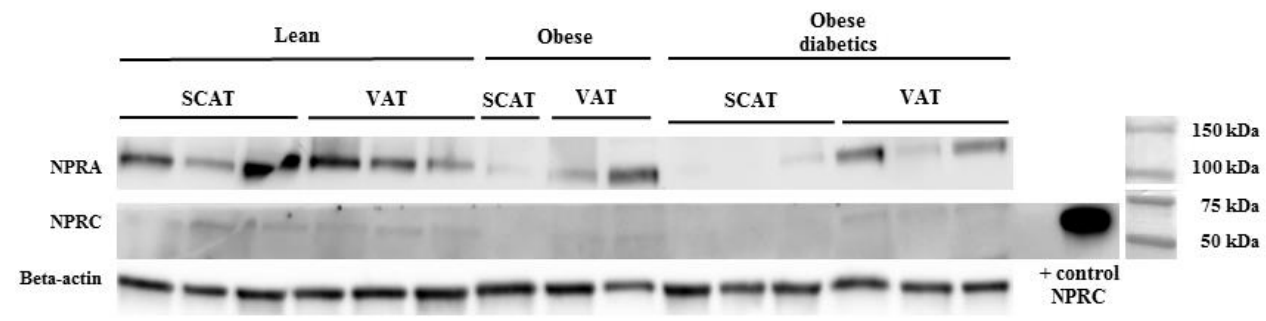

Figure S2 - Validation of NPRC protein expression in SCAT and VAT. In a subset of subjects (lean $\mathrm{n}=3$, obese $\mathrm{n}=5$ ) NPRC protein expression was validated using a positive control (transient overexpression NPRC lysate, $1 \mu \mathrm{g}$ ) showing a band at predicted height $\pm 60-70 \mathrm{kDa}$. 



\section{Chapter 4}

\section{Abdominal subcutaneous and visceral adipocyte morphology, lipolysis and inflammation in relation to insulin resistance in obese humans}

Kenneth Verboven, Kristiaan Wouters, Katrien Gaens, Dominique Hansen, Mitchell Bijnen, Suzan Wetzels, Coen D Stehouwer, Gijs H Goossens, Casper G

Schalkwijk, Ellen E Blaak, Johan WE Jocken 


\section{Abstract}

Aims/hypothesis - Obesity is associated with a disturbed adipose tissue (AT) function characterized by adipocyte hypertrophy, an impaired lipolysis and a pro-inflammatory phenotype, which contributes to insulin resistance (IR). Therefore, we investigated whether AT morphology, lipolysis and inflammation in different AT depots of obese individuals with and without type 2 diabetes mellitus (T2DM) are associated with whole-body IR.

Methods - Subcutaneous (SC) and visceral (V) AT biopsies from age-matched (39-65 year) lean ( $n=19$, BMI $23.9 \pm 0.3 \mathrm{~kg} / \mathrm{m}^{2}$, HOMA-IR $\left.2.1 \pm 0.2\right)$, obese $\left(n=22\right.$, BMI $38.1 \pm 0.9 \mathrm{~kg} / \mathrm{m}^{2}$, HOMA-IR $\left.5.6 \pm 0.7\right)$ and obese T2DM men $(n=11$, BMI $38.1 \pm 1.2 \mathrm{~kg} / \mathrm{m}^{2}$, HOMA-IR $7.9 \pm 1.7$ ) were collected. AT phenotype was characterized by ex vivo measurement of basal, ANP- and isoprenalin-stimulated lipolysis (mature adipocytes), adipocyte size distribution (AT tissue sections) and AT immune cells (flow cytometry).

Results - Adipocyte hypertrophy was present in SCAT and VAT of obese compared to lean individuals. In VAT, CD45+ leukocytes and M1 macrophages were significantly increased in both obese groups compared to lean individuals. In SCAT no significant differences in immune cell populations between groups were found. In SCAT, multiple linear regression analysis, including age, fat mass and AT morphologic, lipolytic and immune cell markers showed that age $(\beta=0.341)$ and total fat mass $(\beta=0.507)$ independently contributed to HOMA-IR (adjusted $\left.R^{2}=0.325\right)$. In VAT, adipocyte size $(\beta=0.619), C D 19+B$ lymphocytes $(\beta=0.286)$ and maximal isoprenalin responsiveness $(\beta=-0.313)$ were significantly related to HOMA-IR, together accounting for $23 \%$ of the variance in HOMA-IR.

Conclusions/interpretation - Adipocyte hypertrophy in both SCAT and VAT is an important phenotype of IR. In hypertrophic visceral adipocytes, M1 macrophage- or B-cell-mediated inflammation may contribute to IR, while inflammation in SCAT does not seem to play a major role in IR.

\section{Introduction}


During the development of obesity, adipose tissue (AT) expansion frequently results in adipocyte hypertrophy (i.e. enlargement of the adipocyte), which is a known stressor for adipocytes ${ }^{1}$. Increases in AT mass and adipocyte volume result in a broad range of metabolic repercussions including, amongst others, a decreased insulin-mediated suppression ${ }^{2,3}$ and attenuated catecholamine or atrial natriuretic peptide (ANP)-mediated stimulation of AT lipolysis ${ }^{4-7}$. This altered AT function is characterized by an impaired lipid buffering capacity, especially in the post-prandial state ${ }^{8}$. Subsequently, this may lead to systemic lipid overflow and ectopic lipid accumulation in several insulin sensitive peripheral tissues like skeletal muscle, liver, pancreas, the heart and kidneys, which relates to the development of peripheral and systemic insulin resistance (IR) ${ }^{9}$.

Different immune cells are activated within hypertrophic AT (in case of resident immune cells) or attracted toward necrotic/apoptotic hypertrophic adipocytes, especially in the visceral AT (VAT), mainly based on rodent studies ${ }^{10,11}$. This VAT inflammation was proposed to be the strongest correlate of IR in human obesity ${ }^{12,13}$. In this regard, AT macrophages have gained much attention as important mediators of AT inflammation. In human obesity, AT macrophages display profound pro-inflammatory (M1) characteristics ${ }^{14,15}$ and are thereby thought to be the major source of pro-inflammatory cytokines and chemokines. This in turn may affect local AT lipolysis and further impede adipocyte function ${ }^{16}$ and reduce insulin sensitivity ${ }^{17-19}$. However, recent evidence indicates the presence of other innate and adaptive immune cells in the AT (reviewed by Mraz et al.) ${ }^{20}$. The cross-talk and dynamics of immune cells initiating and orchestrating AT inflammation and an impaired lipid metabolism in different AT depots are incompletely understood in the obese non-diabetic or type 2 diabetic state.

It is still an ongoing debate whether an impaired AT lipolysis or inflammation, or an interaction between both, is a cause or rather a consequence of IR in the obese state. Animal models propose a causative role of AT inflammation in the pathogenesis of IR ${ }^{21}$. In contrast, recent human studies indicated the development of hyperinsulinemia and dyslipidemia and (peripheral) IR upon overfeeding without affecting the inflammatory phenotype of the SCAT, 
suggesting that AT inflammation is not necessary to evoke peripheral IR in humans ${ }^{22,23}$. Of interest, healthy AT expansion and remodeling implies a certain degree of AT inflammation contrasting the general belief that inflammation negatively affects metabolism ${ }^{24}$.

Therefore, gaining better insights into the association between AT lipolysis, inflammation and IR is imperative to improve future development of therapeutic strategies to ameliorate the adverse metabolic consequences of obesity. In the present study, we studied subcutaneous and visceral AT morphology (adipocyte size), ex vivo lipolysis and immune cell populations (flow cytometry) in relation to whole-body IR in obese non-diabetic and type 2 diabetic (T2DM) men, compared with age-matched lean men. 
Subjects - For this study, lean and obese male age-matched individuals who were scheduled to undergo abdominal or bariatric surgery were recruited, as described previously in more detail ${ }^{7}$. The lean control group consisted of 18 male individuals. The obese group was composed of 30 male individuals, including 19 individuals without type 2 diabetes and 11 individuals with type 2 diabetes. Presence of type 2 diabetes was defined as glycated haemoglobin (HbA1c) $\geq 6.5 \%(45 \mathrm{mmol} / \mathrm{mol})$ or the use of glucose lowering medication based on a clinical diagnosis (on average 2.5 years of diagnosis, ranging from newly diagnosed to 6 years). Major exclusion criteria were the use of exogenous insulin, presence or history of heart, lung or kidney disease and/or presence of endocrine anomalies. The study protocol was approved by the Medical Ethical Committee Jessa hospital, Hasselt, and Hasselt University, Belgium, in accordance with the Declaration of Helsinki (2008), and all individuals gave their written informed consent before participating in the study.

Anthropometric measurements and blood sampling - Body weight, height, waist/hip circumference and blood pressure were determined at the morning of surgery. Fat and lean body mass were estimated by bio-electrical impedance analyses (Bodystat 1500; Bodystat Ltd., Isle of Man, UK). Fasting venous blood samples were collected after an overnight fast for measurement of plasma glucose, serum insulin and $\mathrm{HbA1C}$. Insulin sensitivity was assessed by the homeostatic model assessment index for insulin resistance (HOMA-IR), calculated from fasting glucose and insulin, according to the formula: fasting insulin $(\mathrm{mU} / \mathrm{l}) *$ fasting glucose $(\mathrm{mmol} / \mathrm{l}) / 22.5^{25}$. Plasma glucose concentration was measured by the glucose oxidase method using an AU2700 analyser (Beckman Coulter, Brea, CA, USA). Serum insulin concentration was assessed by immunoassay (ADVIA Centaur Insulin IRI; Siemens Medical Solutions Diagnostics, Tarrytown, NY, USA). HbA1C was assessed by high performance liquid chromatography using a HA-8160 Hi-Auto A1C analyser (Menarini, Zaventem, Belgium).

Abdominal subcutaneous and visceral adipose tissue biopsies - After an overnight fast, adipose tissue biopsies were taken from the abdominal subcutaneous adipose tissue depot (SCAT) and the distal portion of the omentum majus (VAT). The tissue samples were immediately placed in saline 
and transported on ice to the laboratory for further processing. One portion of fresh AT was used for histological sections whereas another portion was used for isolation of the stromal vascular fraction (SVF) and mature adipocytes for lipolysis measurements.

Adipocyte size - A small part of the AT samples was fixed overnight in $4 \%$ paraformaldehyde and embedded in paraffin. Histological sections $(8 \mu \mathrm{m})$ were cut, mounted on microscope glass slides and dried overnight in an incubator $\left(37^{\circ} \mathrm{C}\right)$. Haematoxylin and eosin staining was used. Digital images were captured using a Leica DFC320 digital camera (Leica DM3000 microscope, Leica, Rijswijk, The Netherlands) at 20x magnification. Adipocyte size and distribution was performed in a blinded fashion (coefficient of variation $<5 \%$ ) using computerized morphometric analysis (Leica QWin V3, Cambridge, UK) of individual adipocytes (at least 400 adipocytes per sample), as described previously ${ }^{26}$.

Adipocyte isolation, stromal vascular preparation and lipolysis measurement - Mature adipocytes and stromal vascular cells were obtained, as described previously ${ }^{7}$. Briefly, after collagenase digestion of AT fragments in Dulbecco's modified Eagle's medium (DMEM)-Ham's F12, the resulting suspension was filtered and mature adipocytes were diluted in DMEM-Ham's F12 supplemented with $3 \%$ BSA for lipolysis assays and incubated with isoprenalin (ISO, a non-selective $\beta$-adrenergic agonist; $10-6 \mathrm{M}$ ) or human ANP (Bachem) (10-4 M) for $3 \mathrm{~h}$ at $37^{\circ} \mathrm{C}$. Following incubation, glycerol concentration in the medium (lipolysis index) was determined using the EnzyChromeTM Adipolysis assay kit (Gentaur) and expressed per cell number or as maximal responsiveness to adrenergic or ANP stimulation (compared with baseline).

Flow cytometry - Multicolour flow cytometry measurements were performed as described previously ${ }^{27}$. Briefly, SVF cells, which were obtained following collagenase digestion, were stained for flow cytometry using two antibody cocktails. Samples were measured with a FACS-Canto II (BD Biosciences). Results were analysed with FACSdiva (BD Biosciences) and FlowJo software. Since weight of the AT samples was unavailable, data are expressed as 
percentage of cells relative to total number of cells (based on forward and side scatter plot).

Statistical analyses - All data are presented as mean \pm S.E.M. Shapiro-Wilk tests were used to examine normality and variables with a skewed distribution were In-transformed prior to analysis. Group differences were analysed using one-way ANOVA. Post-hoc testing was performed using Bonferroni adjustment for multiple testing when one-way ANOVA showed a significant group difference. Correlation analysis was applied by Pearson's correlation. Multiple linear regression analyses including age, total fat mass and the AT phenotype markers that were significantly correlated with HOMA-IR (indicated in Table 3) were used to investigate whether AT morphology, lipolysis and inflammation were independently related to whole-body IR (dependent variable, expressed as HOMA-IR). These analyses were performed for both AT depots separately. Statistical significance was set at $p<0.05$ (two-tailed). Analyses were performed using SPSS 22.0 for Windows (SPSS). 


\section{Results}

\section{Subjects' characteristics}

There were no differences in age between lean individuals and both obese groups. As expected, lean individuals' body composition and metabolic parameters, including insulin sensitivity (expressed as HOMA-IR) and glycaemic control (indicated by fasting plasma glucose and HbA1c levels) were significantly different compared to the obese groups (Table 1). BMI, body fat percentage and body fat distribution was not significantly different between both obese groups. Fasting plasma glucose levels, whole-body IR (HOMA-IR) and glycated hemoglobin (HbA1c) were significantly elevated in the obese T2DM individuals as compared to obese non-diabetic individuals (Table 1 ). 


\begin{tabular}{|c|c|c|c|c|}
\hline Variable & $\begin{array}{l}\text { Lean } \\
\text { individuals }\end{array}$ & $\begin{array}{l}\text { Obese insulin resistant } \\
\text { individuals }\end{array}$ & $\begin{array}{l}\text { Obese diabetic } \\
\text { individuals }\end{array}$ & $P_{\text {group }}$ \\
\hline$n$ & 19 & 22 & 11 & \\
\hline Age, years & $52 \pm 1(43-61)$ & $48 \pm 1(39-63)$ & $52 \pm 2(42-65)$ & 0.224 \\
\hline $\mathrm{BMI}, \mathrm{kg} / \mathrm{m}^{2}$ & $23.7 \pm 0.3(20.8-25.6)$ & $38.1 \pm 0.9(30.4-49.2)^{c}$ & $38.1 \pm 1.2(33.8-47.6)^{c}$ & $<0.001$ \\
\hline Body fat, \% & $23.2 \pm 0.9(14.6-30.0)$ & $36.3 \pm 0.9(30.0-46.9)^{c}$ & $36.5 \pm 1.1(29.1-43.1)^{c}$ & $<0.001$ \\
\hline Fat mass, kg & $17.9 \pm 0.8(11.1-24.9)$ & $45.1 \pm 2.6(29.3-72.3)^{c}$ & $42.9 \pm 2.2(33.0-58.0)^{c}$ & $<0.001$ \\
\hline Waist circumference, cm & $90.8 \pm 1.1(78.5-99.0)$ & $128.0 \pm 2.3(111.0-154.0)^{c}$ & $126.1 \pm 2.1(120.5-143.0)^{c}$ & $<0.001$ \\
\hline Hip circumference, cm & $93.3 \pm 0.8(88.5-99.5)$ & $116.8 \pm 2.1(105.0-141.0)^{c}$ & $116.0 \pm 2.1(106.5-127.0)^{c}$ & $<0.001$ \\
\hline Waist-to-hip ratio & $0.97 \pm 0.0(0.89-1.02)$ & $1.09 \pm 0.0(1.03-1.18)^{c}$ & $1.08 \pm 0.0(1.02-1.16)^{c}$ & $<0.001$ \\
\hline Fasting plasma glucose, mmol/l & $5.4 \pm 0.1(2.4-6.6)$ & $5.9 \pm 0.2(4.7-10.1)$ & $7.8 \pm 0.7(5.1-12.0)^{c, d}$ & $<0.001$ \\
\hline Serum insulin, mU/I & $8.7 \pm 0.9(2.1-17.0)$ & $20.9 \pm 2.2(6.0-45.0)^{c}$ & $21.3 \pm 3.2(10.0-40.0)^{c}$ & $<0.001$ \\
\hline $\mathrm{HbA} 1 \mathrm{c}, \%$ & $5.2 \pm 0.0(5.0-5.8)$ & $5.6 \pm 0.1(5.1-7.1)$ & $7.2 \pm 0.3(6.0-9.0)^{c, e}$ & $<0.001$ \\
\hline $\mathrm{HbA} 1 \mathrm{c}, \mathrm{mmol} / \mathrm{mol}$ & $34 \pm 0(31-40)$ & $38 \pm 1(32-54)^{a}$ & $55 \pm 3(42-72)^{c, e}$ & $<0.001$ \\
\hline HOMA-IR & $2.1 \pm 0.2(0.3-4.5)$ & $5.6 \pm 0.7(1.3-14.7)^{c}$ & $7.9 \pm 1.7(2.84-18.0)^{c}$ & $<0.001$ \\
\hline $\begin{array}{l}\text { Subcutaneous adipocyte diameter, } \\
\mu \mathrm{m} \text { (a) }\end{array}$ & $64.5 \pm 2.3(51.7-85.7)$ & $78.6 \pm 2.0(61.0-91.7)^{c}$ & $77.0 \pm 1.9(70.9-86.1)^{b}$ & $<0.001$ \\
\hline $\begin{array}{l}\text { Visceral adipocyte diameter, } \\
\mu \mathrm{m}(\mathrm{a})\end{array}$ & $62.2 \pm 3.8(44.0-85.7)$ & $80.6 \pm 1.9(62.0-91.6)^{c}$ & $81.6 \pm 1.8(72.4-88.1)^{c}$ & $<0.001$ \\
\hline
\end{tabular}

Data are mean \pm S.E.M (range). (a) Variable measured in 15 lean, 20 obese insulin resistant and 9 obese diabetic individuals. Significantly different from lean group $\left({ }^{\mathrm{a}} p<0.05 ;{ }^{\mathrm{b}} p<0.01 ;{ }^{\mathrm{c}} p<0.001\right)$, significantly different from obese insulin resistant group ( ${ }^{\mathrm{d}} p<0.01 ;{ }^{\mathrm{e}} p<0.001$ ) 


\section{SCAT and VAT morphology}

Mean adipocyte size was clearly elevated in both obese groups as compared to the lean group in both SCAT ( $p<0.001$ for obese individuals and $p<0.01$ for obese T2DM) and VAT ( $p<0.001$ for both obese and obese T2DM individuals) (Table 1). However, there was no significant difference in mean adipocyte size between the different AT depots within obese groups (Table 1). The difference in mean adipocyte size between lean and obese individuals was attributed by a shift toward a significantly lower proportion of large $(70-89 \mu \mathrm{m})$ and very large ( $>90 \mu \mathrm{m}$ ) adipocytes, and a higher proportion of small $(<50 \mu \mathrm{m})$ and medium size $(50-69 \mu \mathrm{m})$ adipocytes in the lean individuals, in both the SCAT (Fig. 1A) and VAT (Fig. 1B). Detailed lipolysis data have been reported previously ${ }^{7}$. Briefly, we observed an increased basal lipolysis in the SCAT and VAT of obese and obese diabetic individuals and an attenuated maximal ANP- and ISOinduced lipolysis in the SCAT of obese diabetic men as compared to lean individuals (with intermediate values for obese non-diabetic individuals).
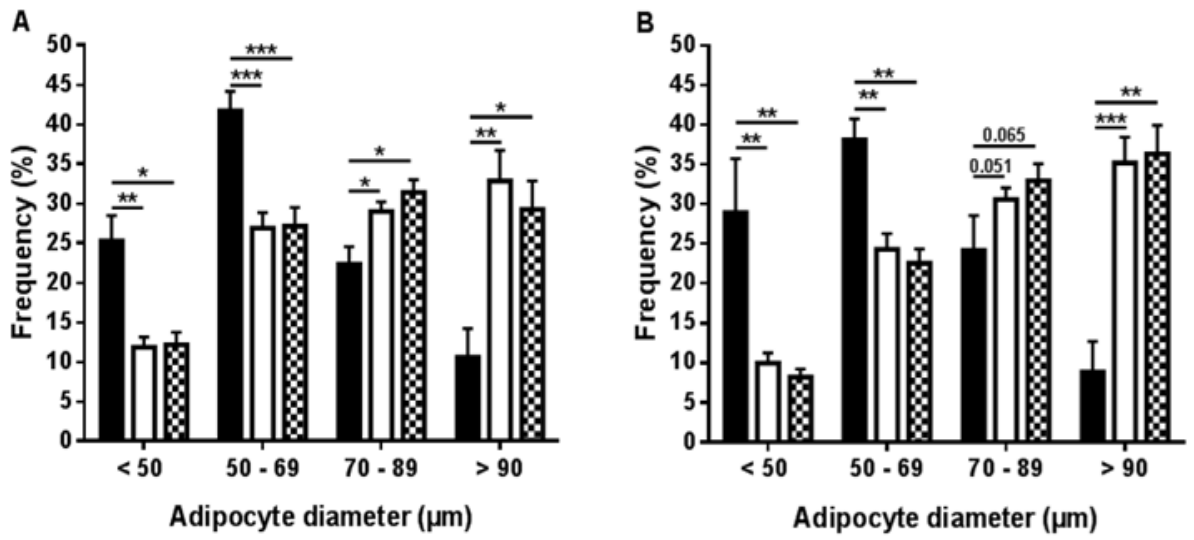

Figure 1 - Adipocyte size distribution. The frequency of small $(<50 \mu \mathrm{m})$, medium $(50-69 \mu \mathrm{m})$, large $(70-89 \mu \mathrm{m})$ and very large $(>90 \mu \mathrm{m})$ adipocytes was determined in SCAT (A) and VAT (B) of lean (black bars), obese insulin resistant (white bars) and obese diabetic (white squared bars) individuals. Obese groups clearly had higher proportions of large and very large adipocytes in combination with smaller proportions of small and medium size adipocytes, resulting in a higher mean adipocytes size compared to lean individuals both in SCAT ( $p<0.001$ for obese insulin resistant individuals and $p<0.01$ for obese diabetic individuals) and VAT $(p<0.001$ for both obese groups) Data are means \pm SEM. * $p<$ $0.05, * * \mathrm{p}<0.01, * * * \mathrm{p}<0.001$. 


\section{Elevated inflammatory cell populations in the SVF of obese visceral AT}

In SCAT, no significant differences were found in immune cell populations, expressed as percentage of total cells, between groups (Table 2). In contrast, the VAT of both obese groups showed a clear increase in proportion of leukocytes (CD45+) numbers, expressed as percentage of total cells, compared to lean controls ( $<<0.001$ for obese individuals and $p<0.01$ for obese T2DM individuals). However, no significant differences were found between groups for the proportion of B-cells (CD19+), T-cell subsets (T-helper cells or cytotoxic Tcells) or NK cells (CD56+) (Table 2). For VAT macrophages, we observed an increased proportion of M1-macrophages (CD11+; expressed as percentage of total cells) in obese non-diabetic individuals $(p<0.05)$ compared to lean controls. A trend toward a higher proportion of CD11- M2 macrophages in obese VAT was observed ( $p_{\text {group }}=0.066$ ) (Table 2 ). No group differences were observed when representing cell subsets as a fraction of total parent cells (either as $\%$ of CD45+ lymphocytes or as \% of macrophages), thereby reflecting qualitative characteristics (Supplemental Table 1). 


\begin{tabular}{|c|c|c|c|c|}
\hline Variable & $\begin{array}{c}\text { Lean } \\
\text { individuals }(n=18) \\
\end{array}$ & $\begin{array}{c}\text { Obese insulin resistant } \\
\text { individuals }(n=18)\end{array}$ & $\begin{array}{l}\text { Obese diabetic individuals } \\
\qquad(n=8)\end{array}$ & $P_{\text {group }}$ \\
\hline \multicolumn{5}{|l|}{ Subcutaneous SVF } \\
\hline Total CD45+ leukocytes & $41.3 \pm 4.2(4.3-70.4)$ & $44.2 \pm 4.0(18.0-74.0)$ & $38.7 \pm 5.5(23.5-74.0)$ & 0.862 \\
\hline B cells & $0.79 \pm 0.16(0.10-2.44)$ & $1.26 \pm 0.32(0.14-5.20)$ & $1.08 \pm 0.36(0.17-3.00)$ & 0.300 \\
\hline $\mathrm{T}$ cells & $21.9 \pm 2.7(1.8-48.9)$ & $25.1 \pm 1.8(7.3-35.7)$ & $24.9 \pm 3.1(16.3-42.3)$ & 0.600 \\
\hline CD4+ T helper cells & $12.0 \pm 2.0(0.8-33.3)$ & $11.9 \pm 1.0(3.3-19.9)$ & $14.0 \pm 2.5(7.1-28.1)$ & 0.768 \\
\hline CD8+ cytotoxic T cells & $6.5 \pm 0.7(0.6-10.8)$ & $8.0 \pm 0.7(2.5-11.5)$ & $7.7 \pm 1.1(3.8-13.1)$ & 0.334 \\
\hline CD4+/CD8+ ratio & $1.8 \pm 0.2(0.4-3.7)$ & $1.5 \pm 0.1(0.8-2.9)$ & $1.95 \pm 0.2(0.5-2.9)$ & 0.764 \\
\hline NK cells & $2.7 \pm 0.5(0.1-8.1)$ & $3.7 \pm 0.3(1.8-6.1)$ & $4.1 \pm 1.0(0.5-7.8)$ & 0.211 \\
\hline M1 macrophages & $6.6 \pm 1.2(0.2-16.1)$ & $10.7 \pm 1.2(0.2-19.6)$ & $5.6 \pm 1.4(0.3-12.0)$ & 0.076 \\
\hline M2 macrophages & $5.1 \pm 1.0(0.4-15.5)$ & $5.9 \pm 1.5(0.6-22.8)$ & $3.3 \pm 0.9(0.3-8.3)$ & 0.706 \\
\hline M1/M2 ratio & $2.1 \pm 0.6(0.4-11.5)$ & $3.3 \pm 0.6(0.3-8.6)$ & $2.4 \pm 0.8(0.5-8.2)$ & 0.298 \\
\hline \multicolumn{5}{|l|}{ Visceral SVF } \\
\hline Total CD45+ leukocytes & $34.9 \pm 3.5(5.6-54.3)$ & $57.3 \pm 3.0(33.0-93.8)^{c}$ & $53.5 \pm 2.6(41.6-65.7)^{b}$ & $<0.001$ \\
\hline B cells & $1.7 \pm 0.5(0.1-7.5)$ & $3.9 \pm 1.6(0.0-30.4)$ & $2.2 \pm 0.9(0.7-8.7)$ & 0.377 \\
\hline T cells & $28.7 \pm 3.3(3.3-59.3)$ & $36.3 \pm 3.2(15.1-56.2)$ & $33.9 \pm 5.2(12.7-51.4)$ & 0.394 \\
\hline CD4+ T helper cells & $15.3 \pm 2.1(1.7-31.8)$ & $17.7 \pm 1.7(0.3-28.6)$ & $17.2 \pm 2.7(6.6-28.9)$ & 0.745 \\
\hline CD8+ cytotoxic T cells & $10.1 \pm 1.2(0.9-18.3)$ & $13.7 \pm 1.8(3.3-25.0)$ & $13.5 \pm 3.0(0.9-26.4)$ & 0.265 \\
\hline CD4+/CD8+ ratio & $1.7 \pm 0.2(0.7-5.2)$ & $1.7 \pm 0.3(0.0-5.8)$ & $2.7 \pm 1.3(0.5-12.3)$ & 0.695 \\
\hline NK cells & $3.0 \pm 0.4(0.0-7.4)$ & $3.5 \pm 0.5(0.2-8.9)$ & $4.0 \pm 1.0(0.4-8.6)$ & 0.725 \\
\hline M1 macrophages & $2.9 \pm 0.5(0.1-9.0)$ & $6.3 \pm 0.9(1.3-12.7)^{a}$ & $5.1 \pm 0.9(1.2-8.9)$ & 0.010 \\
\hline M2 macrophages & $3.5 \pm 0.5(0.1-8.5)$ & $7.4 \pm 1.4(0.6-24.1)$ & $7.2 \pm 1.7(0.9-17.0)$ & 0.066 \\
\hline $\mathrm{M} 1 / \mathrm{M} 2$ ratio & $0.9 \pm 0.1(0.2-2.2)$ & $1.4 \pm 0.3(0.1-5.3)$ & $1.2 \pm 0.4(0.2-4.3)$ & 0.725 \\
\hline
\end{tabular}

Data are mean \pm S.E.M (range). Cell frequencies are expressed as \% of total cells; NK, natural killer; CD, cluster of differentiation; SVF,

stromal vascular fraction. Significantly different from lean group $\left({ }^{\mathrm{a}} p<0.05 ;{ }^{\mathrm{b}} p<0.01 ;{ }^{\mathrm{c}} p<0.001\right)$ 
Table 3 Pearson correlation coefficients between AT lipolysis, AT size and AT immunophenotype of subcutaneous and visceral AT depots and whole-body IR

\section{Subcutaneous AT}

Basal lipolysis, $\mu \mathrm{mol}$ glycerol (3h)

per $10^{6}$ cells

Isoprenalin responsiveness, fold change

to basal

ANP responsiveness, fold change

to basal

Adipocyte diameter, $\mu \mathrm{m}$

Total CD45+ leukocytes, \% of total cells

CD3+ $T$ cells, $\%$ of total cells

CD3+CD4+ T-helper cells, \% of live cells

CD3+CD8+ cytotoxic T cells, \% of total cells

$\mathrm{CD} 4+/ \mathrm{CD} 8+\mathrm{T}$ cell ratio

CD56+ NK cells, \% of total cells

CD11+ M1 macrophages, \% of total cells

CD11- M2 macrophages, \% of total cells

$\mathrm{M} 1 / \mathrm{M} 2$ ratio

CD19+ B lymphocytes, \% of total cells

\begin{tabular}{|c|c|c|c|c|}
\hline $\mathbf{R}$ & p value & Visceral AT & $\mathbf{R}$ & p value \\
\hline 0.526 & 0.001 & $\begin{array}{l}\text { Basal lipolysis, } \mu \mathrm{mol} \text { glycerol }(3 \mathrm{~h}) \\
\text { per } 10^{6} \text { cells }\end{array}$ & 0.126 & 0.444 \\
\hline-0.249 & 0.126 & $\begin{array}{l}\text { Isoprenalin responsiveness, fold change } \\
\text { to basal }\end{array}$ & -0.364 & 0.023 \\
\hline-0.176 & 0.284 & $\begin{array}{l}\text { ANP responsiveness, fold change } \\
\text { to basal }\end{array}$ & -0.187 & 0.261 \\
\hline 0.460 & 0.002 & Adipocyte diameter, $\mu \mathrm{m}$ & 0.603 & $<0.001$ \\
\hline 0.186 & 0.243 & Total CD45+ leukocytes, \% of total cells & 0.515 & $<0.001$ \\
\hline 0.389 & 0.014 & CD3+ T cells, $\%$ of total cells & 0.143 & 0.366 \\
\hline 0.218 & 0.182 & CD3+CD4+ T-helper cells, \% of total cells & 0.008 & 0.960 \\
\hline 0.506 & 0.001 & CD3+CD8+ cytotoxic T cells, $\%$ of total cells & 0.254 & 0.110 \\
\hline-0.067 & 0.685 & $\mathrm{CD} 4+/ \mathrm{CD} 8+\mathrm{T}$ cell ratio & -0.083 & 0.605 \\
\hline 0.452 & 0.004 & CD56+ NK cells, $\%$ of live cells & 0.157 & 0.320 \\
\hline 0.062 & 0.701 & CD11+ M1 macrophages, $\%$ of total cells & 0.261 & 0.095 \\
\hline-0.037 & 0.819 & CD11- M2 macrophages, \% of total cells & 0.217 & 0.168 \\
\hline 0.059 & 0.716 & $\mathrm{M} 1 / \mathrm{M} 2$ ratio & 0.042 & 0.792 \\
\hline 0.174 & 0.289 & CD19+ B lymphocytes, \% of total cells & 0.373 & 0.015 \\
\hline
\end{tabular}

ANP, atrial natriuretic peptide; AT, adipose tissue; NK, natural killer; $C D$, cluster of differentiation 


\section{Determinants of whole-body insulin resistance}

First, we investigated whether AT functional variables (adipocyte size, basal lipolysis and maximal lipolytic responsiveness to ISO or ANP) and AT immunophenotype were associated with whole-body IR, estimated using HOMAIR (Table 3). In SCAT, basal lipolysis (expressed per cell number; $p=0.001$ ) and mean adipocyte size $(p=0.002)$ were positively correlated with HOMA-IR. Furthermore, SCAT CD3+ T-cells $(p=0.014)$, CD8+ cytotoxic T-cells $(p=0.001)$ and CD56+ NK-cells $(p=0.004)$ correlated positively with HOMA-IR (Table 3 ). In VAT, mean adipocyte size correlated positively $(p<0.001)$, whereas maximal ISO responsiveness (fold change compared to basal) was negatively correlated $(p=0.023)$ with HOMA-IR. Moreover, VAT CD45+ leukocytes $(p<0.001)$ and CD19+ B-cells $(p=0.015)$ were positively correlated with HOMA-IR, while VAT CD11+ M1-macrophages tended to show a positive association with HOMA-IR $(r=0.26, p=0.095)$ (Table 3). Of interest, AT functional variables and AT immunophenotype showed some mutual correlations in the SCAT as well as in the VAT, respectively (Supplemental Table 2).

The variables significantly correlated with HOMA-IR (marked associations in Table 3) were subsequently analysed in a multiple linear regression analyses for both AT depots. In SCAT, only age $(\beta=0.341, p=0.037)$ and total fat mass $(\beta=0.507, p=0.022)$ were significantly related to HOMA-IR and together explained $32.5 \%$ of the variance in HOMA-IR. In VAT, adipocyte size $(\beta=0.619$, $p=0.001)$, maximal ISO responsiveness $(\beta=-0.313, p=0.016)$ and CD19+ $B$ cells $(\beta=0.286, p=0.039)$ accounted for $23 \%$ of the variance in HOMA-IR $\left(p_{\text {model }}<0.001\right)$. 


\section{Discussion}

To the best of our knowledge, this is the first study reporting detailed data on the relationship between depot-specific AT phenotype (i.e. morphology, lipolysis and inflammation) and whole-body IR (HOMA-IR) in lean, obese non-diabetic individuals and obese humans with type 2 diabetes. As reported previously ${ }^{7}$, obese individuals have an attenuated basal lipolysis in both SCAT and VAT, and a decreased maximal ANP- and ISO-mediated lipolytic responsiveness in SCAT, compared to age-matched lean individuals. Here we demonstrate that this altered AT phenotype was further characterized by adipocyte hypertrophy and an increased immune cell infiltration, especially in the VAT. Of interest, in the VAT adipocyte size and CD19+ B cells were positively related to whole-body IR, while maximal ISO responsiveness was negatively related to whole-body IR. In contrast, none of the subcutaneous AT functional measures or immunophenotype were independently related to whole-body IR.

The present findings indicate that adipocyte hypertrophy in the obese SCAT and VAT is related to whole-body IR, which is in line with previous studies ${ }^{28-30}$. In addition, VAT adipocyte hypertrophy was independently and positively associated $(\beta=0.619, p=0.001)$ with whole-body $I R$, also after adjustment for age and total fat mass. Although adipocyte hypertrophy is the preferential expansion mechanism within the AT, in the chronic state of obesity SCAT adipogenesis is commonly impaired ${ }^{31}$, possibly resulting in lipid spill-over to other tissues (the VAT as well) and thus the development of metabolic complications ${ }^{32}$. The latter may explain our finding that adipocyte hypertrophy in VAT was an independent determinant of whole-body IR. Importantly, abdominal subcutaneous adipocyte hypertrophy may thus substantially contribute to expansion of VAT, which in turn results in worsening of whole-body IR.

The presence of a chronic pro-inflammatory AT microenvironment may limit adipocyte hypertrophy, by activating fibrotic signaling and impairing adipocyte functionality ${ }^{33}$, thereby contributing to metabolic alterations frequently observed in the obese state ${ }^{34,35}$. In addition to adipocyte hypertrophy, lowgrade AT inflammation is essential for healthy AT expansion and remodeling, as 
some cells are implicated in the clearance of cell debris released by necrotic adipocytes ${ }^{36}$, act as a local buffer against increased lipid content ${ }^{37}$, and promote angiogenesis ${ }^{38}$. In this regard, our data show a significantly increased proportion of CD45+ leukocytes and CD11+ M1 macrophages in the VAT of obese and obese diabetic humans. In contrast, an inflammatory phenotype was less pronounced in the SCAT of obese individuals compared to lean or obese T2DM individuals, where we only observed a trend toward a higher proportion of CD11+ M1 (pro-inflammatory) macrophages in the obese SCAT (Table 2). Additionally, no immune-related markers in SCAT were significantly related to HOMA-IR, when adjusting for total fat mass and age. Indeed, the VAT is believed to have a more pronounced inflammatory phenotype than the SCAT ${ }^{39}$, which may possibly be ascribed to an increased production of inflammatory mediators by adipocytes themselves and by cells in the SVF ${ }^{40}$. Concerning the latter, one should be aware that SCAT is more important in quantitative terms, since SCAT accounts for approximately $80 \%$ of total AT mass. This may also explain why total fat mass rather than adipocyte morphology or the SCAT function was the strongest independent determinant of whole-body IR.

Therefore, in the VAT, the potential involvement of the elevated immune cells (i.e. CD45+ leukocytes and CD11+ M1 macrophages) in the development of AT inflammation and IR in obese humans is likely, indicated by a strong positive correlation between these parameters and HOMA-IR (Table 3). Notably, in the multiple linear regression analyses, VAT CD19+ $B(\beta=0.286, p=0.039)$ cells were independently associated with HOMA-IR, also after adjustment for age and total fat mass. B lymphocytes are known to be elevated in obesity and IR, at least in diet-induced obese mice ${ }^{41}$. In epididymal AT, by orchestrating $T$ cell populations and their activity (i.e. production of pro-inflammatory cytokines) and the release of pathogenic antibodies, $\mathrm{B}$ cells can decrease glucose tolerance 41. Recently, the pro-inflammatory features of $B$ cells in a high-fat diet setting were confirmed in obese mice ${ }^{42}$. These data, together with the present findings, suggest that B cells exert pro-inflammatory effects in obese AT and may contribute to IR. However, the precise mechanisms via which these cells impair insulin sensitivity require further investigation, especially in human obesity. 
Inflammatory cells may have a modulatory function in chronically increased lipolysis. Indeed, beside the presence of inflammation additional mechanisms that may be (in)directly related to inflammation may be of importance in worsening of IR in obesity, as previously suggested ${ }^{43}$. Of interest, Kosteli et al. 37 suggested that AT macrophages may buffer excess intracellular fatty acids upon increased lipolysis during fasting. The chronic elevation and uptake of lipids by macrophages may alter their phenotype ${ }^{44}$, promoting the progression to an inflammatory (CD11+) M1 polarization and AT inflammation ${ }^{45}$. However, in the present study no association between SCAT and VAT immunophenotype and measures of lipolysis were observed, suggesting that adipocyte lipolysis does not activate inflammatory pathways in human AT macrophages, as shown recently ${ }^{46}$. In line, only in the VAT a correlation between M1 and M2 macrophages and adipocyte size was found (Supplemental Table 2), respectively, suggesting that different immunological processes or alternative stimuli for AT macrophages (e.g. concentration of saturated fatty acids or ratio of monounsaturated to saturated fatty acids) could be present between abdominal SCAT and VAT depots in humans. Moreover, altered AT oxygen tension may have a putative role in AT dysfunction in obese insulin resistant conditions, as recently reviewed ${ }^{47}$. However, further studies are warranted to obtain more insight into the triggers of AT dysfunction within different AT depots.

Future studies should include human mechanistic experiments to strengthen these findings by focusing on intra-AT relationships and to address causality. In addition, it needs to be mentioned that a significant proportion of the obese patients in the present study received several forms of glucose lowering ( $72 \%$ of the obese diabetic men) or lipid lowering ( $22 \%$ of the obese insulin resistant men; $36 \%$ of the obese diabetic men) medication, which may impact the AT phenotype and have cofounded the present results ${ }^{48,49}$. Furthermore, the use of HOMA-IR as a surrogate markers to assess whole-body IR, may be a limitation of this study. It would be of interest to relate AT functional characteristics to tissue-specific (e.g. muscle, AT or liver) IR, and future studies should therefore use a two-step hyperinsulinemic-euglycemic clamp combined with a glucose tracer. 


\section{Conclusion}

In conclusion, this study is the first to characterize the SCAT and VAT phenotype in detail in lean, obese and obese type 2 diabetic subjects. In VAT, adipocyte hypertrophy independently contributes to IR, possibly via pro-inflammatory M1 macrophage- and/or B cell-mediated inflammation. In SCAT, AT function or immunophenotype were only of minor importance with respect to whole-body IR. These alterations in AT mass and phenotypes likely impact cardiometabolic risk and may thus contribute to the development of type 2 diabetes.

\section{Acknowledgements}

We thank all abdominal surgeons from the Jessa Hospital (Hasselt, Belgium) and Hospital East-Limburg (Genk, Belgium) for their contributions to subject recruitment. We thank University Biobank Limburg (UBiLim, Jessa Hospital, Belgium) for their technical support in sample handling and storage. Yvonne Essers, Nicole Hoebers, Maria Vroomen and José van de Gaar are greatly acknowledged for their technical support. 


\section{References}

1. Khan T, Muise ES, Iyengar P, Wang ZV, Chandalia M, Abate N et al (2009) Metabolic dysregulation and adipose tissue fibrosis: role of collagen VI. Mol Cell Biol 29:15751591

2. Jocken JW, Langin D, Smit E, Saris WH, Valle C, Hul GB et al (2007) Adipose triglyceride lipase and hormone-sensitive lipase protein expression is decreased in the obese insulin-resistant state. J Clin Endocrinol Metab 92:2292-2299

3. Bickerton AS, Roberts R, Fielding BA, Tornqvist H, Blaak EE, Wagenmakers AJ et al (2008) Adipose tissue fatty acid metabolism in insulin-resistant men. Diabetologia 51:1466-1474

4. Jocken, JW, Blaak EE, van der Kallen CJ, van Baak MA, Saris WH (2008) Blunted betaadrenoceptor-mediated fat oxidation in overweight subjects: a role for the hormonesensitive lipase gene. Metabolism 57:326-332

5. Nielsen TS, Jessen N, Jørgensen JO, Møller N, Lund S (2014) Dissecting adipose tissue lipolysis: molecular regulation and implications for metabolic disease. J Mol Endocrinol 52:R199-222

6. Rydén M, Bäckdahl J, Petrus P, Thorell A, Gao H, Coue M et al (2015) Impaired atrial natriuretic peptide-mediated lipolysis in obesity. Int J Obes (London) 40:714-720

7. Verboven K, Hansen D, Moro C, Eijnde BO, Hoebers N, Knol J et al (2016) Attenuated atrial natriuretic peptide-mediated lipolysis in subcutaneous adipocytes of obese type 2 diabetic men. Clin Sci (London) 130:1105-1114

8. McQuaid SE, Hodson L, Neville MJ, Dennis AL, Cheeseman J, Humphreys SM (2011) Downregulation of adipose tissue fatty acid trafficking in obesity: a driver for ectopic fat deposition? Diabetes 60:47-55

9. Stinkens R, Goossens GH, Jocken JW, Blaak EE (2015) Targeting fatty acid metabolism to improve glucose metabolism. Obes Rev 16:715-757

10. Osborn O, Olefsky JM (2012) The cellular and signaling networks linking the immune system and metabolism in disease. Nat Med 18:363-374

11. Mathis D (2013) Immunological goings-on in visceral adipose tissue. Cell Metab 17:851-859

12. Klöting N, Fasshauer M, Dietrich A, Kovacs $P$, Schön MR, Kern M (2010) Insulinsensitive obesity. Am J Physiol Endocrinol Metab 299:E506-E515

13. Hardy OT, Perugini RA, Nicoloro SM, Gallagher-Dorval K, Puri V, Straubhaar J et al (2011) BMI-independent inflammation in omental adipose tissue associated with insulin resistance in morbid obesity. Surg Obes Relat Dis 7:60-67

14. Lumeng CN, Bodzin JL, Saltiel AR (2007) Obesity induces a phenotypic switch in adipose tissue macrophage polarization. J Clin Invest 117:175-184

15. Lumeng CN, DelProposto JB, Westcott DJ, Saltiel AR (2008) Phenotypic switching of adipose tissue macrophages with obesity is generated by spatiotemporal differences in macrophage subtypes. Diabetes 57:3239-3246

16. Langin D (2006) Adipose tissue lipolysis as a metabolic pathway to define pharmacological strategies against obesity and the metabolic syndrome. Pharmacol Res 53:482-491

17. Olefsky JM, Glass CK (2010) Macrophages, inflammation and insulin resistance. Annu Rev Physiol 72:219-246

18. Ouchi N, Parker JL, Lugus JJ, Walsh K (2011) Adipokines in inflammation and metabolic disease. Nat Rev Immunol 11:85-97

19. Glass CK, Olefsky JM (2012) Inflammation and lipid signaling in the etiology of insulin resistance. Cell Metab 15:635-645 
20. Mraz M, Haluzik M (2014) The role of adipose tissue immune cells in obesity and lowgrade inflammation. J Endocrinol 222:R113-127

21. Xu H, Barnes GT, Yang Q, Tan G, Yang D, Chou CJ et al (2003) Chronic inflammation in fat plays a crucial role in the development of obesity-related insulin resistance. J Clin Invest 112:1821-1830

22. Tam CS, Viardot A, Clément K, Tordjman J, Tonks K, Greenfield JR et al (2010) Shortterm overfeeding may induce peripheral insulin resistance without altering subcutaneous adipose tissue macrophages in humans. Diabetes 59:2164-2170

23. Johannsen DL, Tchoukalova Y, Tam CS, Covington JD, Xie W, Schwarz JM et al (2014) Effect of 8 weeks of overfeeding on ectopic fat deposition and insulin sensitivity: testing the adipose tissue expandability hypothesis. Diabetes Care 37:2789-2797

24. Blüher M (2016) Adipose tissue inflammation: a cause or consequence of obesityrelated insulin resistance? Clin Sci (London) 130:1603-1614

25. Matthews DR, Hosker JP, Rudenski AS, Naylor BA, Treacher DF, Turner RC (1985) Homeostasis model assessment: insulin resistance and beta-cell function from fasting plasma glucose and insulin concentration in man. Diabetologia 28:412-419

26. Goossens GH, Bizzarri A, Venteclef N, Essers Y, Cleutjens JP, Konings E et al (2011) Increased adipose tissue oxygen tension in obese compared with lean men is accompanied by insulin resistance, impaired adipose tissue capillarization, and inflammation. Circulation 124:67-76

27. Wouters K, Gaens K, Bijnen M, Verboven K, Jocken JW, Wetzels S et al (2017) Circulating classical monocytes are associated with CD11c+ macrophages in human visceral adipose tissue. Sci Rep 7, 42665; doi: 10.1038/srep42665

28. Weyer C, Foley JE, Bogardus C, Tataranni PA, Pratley RE (2000) Enlarged subcutaneous abdominal adipocyte size, but not obesity itself, predicts type 2 diabetes independent of insulin resistance. Diabetologia 43:1498-1506

29. Lönn M, Mechlig K, Bengtsson C, Lissner L (2010) Adipocyte size predicts incidence of type 2 diabetes in women. FASEB J 24:326-331

30. Acosta JR, Douagi I, Andersson DP, Bäckdahl J, Rydén M, Arner P, Laurencikine J (2016) Increased fat cell size: a major phenotype of subcutaneous white adipose tissue in non-obese individuals with type 2 diabetes. Diabetologia 59:560-570

31. Kim SM, Lun M, Wang M, Senyo SE, Guillermier C Patwari P et al (2014) Loss of white adipose hyperplastic potential is associated with enhanced susceptibility to insulin resistance. Cell Metab 20:1049-1058

32. Pellegrinelli V, Carobbio S, Vidal-Puig A (2016) Adipose tissue plasticity: how fat depots respond differently to pathophysiological cues. Diabetologia 59:1075-1088

33. Abdennour M, Reggio S, Le Naour G, Liu Y, Poitou C, Aron-Wisnewsky J et al (2014) Association of adipose tissue and liver fibrosis with tissue stiffness in morbid obesity: links with diabetes and BMI loss after gastric bypass. J Clin Endocrinol Metab 99:898907

34. Strissel KJ, Stancheva Z, Miyoshi H, Perfield JW, DeFuria J, Jick Z et al (2007) Adipocyte death, adipose tissue remodeling and obesity complications. Diabetes 56:2910-2918

35. Pellegrinelli V, Heuvingh J, du Roure O, Rouault C, Devulder A, Klein C et al (2014) Human adipocyte function is impacted by mechanical cues. J Pathol 233:183-195

36. Cinti S, Mitchell G, Barbatelli G, Murano I, Ceresi E, Faloia E et al (2005) Adipocyte death defines macrophage localization and function in adipose tissue of obese mice and humans. J Lipid Res 46:2347-2355 
37. Kosteli A, Sugaru E, Haemmerle G, Martin JF, Lei J, Zechner R et al (2010) Weight loss and lipolysis promote a dynamic immune response in murine adipose tissue. J Clin Invest 120:3466-3479

38. Cao Y (2013) Angiogenesis and vascular functions in modulation of obesity, adipose metabolism, and insulin sensitivity. Cell Metabolism 18:478-489

39. Hamdy O, Porramatikul S, Al-Ozairi E (2006) Metabolic obesity: the paradox between visceral and subcutaneous fat. Curr Diabetes Rev 2:367-373

40. Fain JN (2010) Release of inflammatory mediators by human adipose tissue is enhanced in obesity and primarily by the nonfat cells - a review. Mediators Inflamm doi: $10.1155 / 2010 / 513948$

41. Winer DA, Winer S, Shen L, Wadia PP, Yantha J, Paltser G et al (2011) B lymphocytes promote insulin resistance through modulation of $\mathrm{T}$ lymphocytes and production of pathogenic IgG antibody. Nat Med 17:610-617

42. Defuria J, Belkina AC, Jagannathan-Bogdan M, Snyder-Cappione J, Carr JD, Nersesova YR et al (2013) B cells promote inflammation in obesity and type 2 diabetes through regulation of $\mathrm{T}$ cell function and an inflammatory cytokine profile. Proc Natl Acad Sci USA 110:5133-5138

43. Barbarroja N, Lopez-Pedrera C, Garrido-Sanchez L, Mayas MD, Olivia-Olivera W, Bernal-Lopez MR et al (2012) Progression from high insulin resistance to type 2 diabetes does not entail additional visceral adipose tissue inflammation. PLoS One 7:e48155

44. Klein-Wieringa IR, Andersen SN, Kwekkeboom JC, Giera M, de Lange-Brokaar BJ, van Osch GJ et al (2013) Adipocytes modulate the phenotype of human macrophages through secreted lipids. J Immunol 191:1356-1363

45. Prieur X, Mok CY, Velagapudi VR, Nunez V, Fuentes L, Montaner D et al (2011) Differential lipid partitioning between adipocytes and tissue macrophages modulates macrophage lipotoxicity and M2/M1 polarization in obese mice. Diabetes 60:797-809

46. Goossens GH, Blaak EE (2012) Adipose tissue oxygen tension: implications for chronic metabolic and inflammatory diseases. Curr Opin Clin Nutr Metab Care 15:539-546

47. Kothari V, Galdo JA, Mathews ST (2016) Hypoglycemic agents and potential antiinflammatory activity. J Inflamm Res 9:27-38

48. Welty FK, Alfaddagh A, Elajami TK (2016) Targeting inflammation in metabolic syndrome. Transl Res 167:257-280 



\section{Supplemental data}

Supplemental Table 1 Frequency of cell populations in subcutaneous and visceral SVF, expressed as percentage relative to $\mathrm{CD}^{+} 5^{+}$leukocytes or to total macrophages

\begin{tabular}{|c|c|c|c|c|}
\hline Variable & $\begin{array}{c}\text { Lean } \\
\text { individuals }(n=18)\end{array}$ & $\begin{array}{c}\text { Obese insulin resistant } \\
\text { individuals }(n=18)\end{array}$ & $\begin{array}{l}\text { Obese diabetic } \\
\text { individuals }(n=8)\end{array}$ & $\mathrm{P}_{\text {group }}$ \\
\hline \multicolumn{5}{|l|}{ Subcutaneous SVF } \\
\hline B cells ( $\%$ of $\left.C D 45^{+}\right)$ & $1.8 \pm 0.2(0.5-4.5)$ & $2.0 \pm 0.4(0.4-7.2)$ & $1.8 \pm 0.5(0.4-4.2)$ & 0.901 \\
\hline T cells $\left(\%\right.$ of $C D 45^{+}$) & $50.1 \pm 2.3(32.4-69.4)$ & $41.4 \pm 3.3(14.9-67.5)$ & $46.2 \pm 2.8(33.3-55.5)$ & 0.094 \\
\hline CD4+ T helper cells ( $\%$ of $C D 45^{+}$) & $25.9 \pm 2.1(10.1-42.6)$ & $19.6 \pm 1.7(1.8-29.1)$ & $25.5 \pm 3.2(14.1-39.2)$ & 0.073 \\
\hline CD8+ cytotoxic $\mathrm{T}$ cells $\left(\%\right.$ of $\left.\mathrm{CD} 45^{+}\right)$ & $16.4 \pm 1.6(8.1-39.8)$ & $13.4 \pm 1.5(1.8-28.0)$ & $14.4 \pm 1.8(8.7-26.0)$ & 0.398 \\
\hline NK cells $\left(\%\right.$ of $\left.C D 45^{+}\right)$ & $6.3 \pm 0.9(0.7-17.8)$ & $7.1 \pm 0.9(2.4-20.0)$ & $7.1 \pm 1.4(1.1-11.6)$ & 0.814 \\
\hline M1 macrophages (\% of macrophages) & $54.4 \pm 5.1(12.8-93.8)$ & $63.3 \pm 4.9(5.2-83.9)$ & $55.0 \pm 8.3(20.5-85.5)$ & 0.431 \\
\hline M2 macrophages (\% of macrophages) & $45.5 \pm 5.1(6.1-87.1)$ & $36.6 \pm 4.9(16.0-94.7)$ & $44.9 \pm 8.3(14.4-79.4)$ & 0.431 \\
\hline \multicolumn{5}{|l|}{ Visceral SVF } \\
\hline B cells ( $\%$ of $\left.C D 45^{+}\right)$ & $3.6 \pm 1.3(0.3-23.0)$ & $5.0 \pm 1.8(0.0-34.2)$ & $3.6 \pm 1.3(1.0-13.0)$ & 0.777 \\
\hline T cells ( $\%$ of $C D 45^{+}$) & $58.7 \pm 2.7(43.4-77.7)$ & $52.5 \pm 4.2(17.0-83.0)$ & $53.4 \pm 6.8(21.3-69.8)$ & 0.453 \\
\hline CD4+ T helper cells ( $\%$ of $C D 45^{+}$) & $30.9 \pm 2.0(16.7-46.1)$ & $25.8 \pm 2.6(0.6-46.4)$ & $26.6 \pm 3.3(14.7-41.6)$ & 0.288 \\
\hline CD8+ cytotoxic $\mathrm{T}$ cells $\left(\%\right.$ of $\left.\mathrm{CD} 45^{+}\right)$ & $20.9 \pm 1.6(8.7-33.1)$ & $19.1 \pm 2.4(0.0-34.7)$ & $21.4 \pm 4.3(1.4-39.9)$ & 0.787 \\
\hline NK cells ( $\%$ of $\left.C D 45^{+}\right)$ & $6.6 \pm 1.0(0.0-16.1)$ & $5.2 \pm 0.9(0.3-15.5)$ & $7.3 \pm 2.2(0.6-19.0)$ & 0.503 \\
\hline M1 macrophages (\% of macrophages) & $45.3 \pm 3.8(19.4-79.4)$ & $51.6 \pm 5.0(11.1-84.1)$ & $46.4 \pm 7.1(23.5-81.2)$ & 0.596 \\
\hline M2 macrophages (\% of macrophages) & $54.6 \pm 3.8(20.5-80.5)$ & $48.3 \pm 5.0(15.8-88.8)$ & $53.5 \pm 7.1(18.7-76.4)$ & 0.596 \\
\hline
\end{tabular}

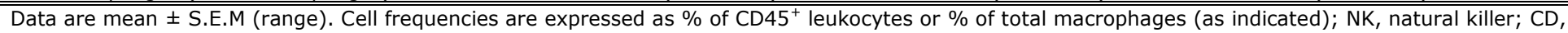
cluster of differentiation; SVF, stromal vascular fraction. 
Pearson correlation coefficients between adipocyte size and lipolysis with immuno-phenotype in subcutaneous and visceral adipose tissue

\begin{tabular}{|c|c|c|c|c|c|}
\hline Subcutaneous adipocyte size ( $\mu \mathrm{m})$ & $\mathbf{R}$ & p value & Visceral adipocyte size $(\mu \mathrm{m})$ & $\mathbf{R}$ & p value \\
\hline Total CD45+ leukocytes, \% of total cells & 0.172 & 0.308 & Total CD45+ leukocytes, \% of total cells & 0.669 & $<0.001$ \\
\hline $\mathrm{CD} 3+\mathrm{T}$ cells, $\%$ of total cells & 0.269 & 0.118 & CD3+ T cells, $\%$ of total cells & 0.222 & 0.157 \\
\hline CD3+CD4+ T-helper cells, \% of total cells & 0.143 & 0.412 & CD3+CD4+ T-helper cells, \% of total cells & 0.203 & 0.196 \\
\hline $\mathrm{CD} 3+\mathrm{CD} 8+$ cytotoxic T cells, \% of total cells & 0.381 & 0.024 & $\mathrm{CD} 3+\mathrm{CD} 8+$ cytotoxic $\mathrm{T}$ cells, \% of total cells & 0.168 & 0.295 \\
\hline $\mathrm{CD} 4+/ \mathrm{CD} 8+\mathrm{T}$ cell ratio & -0.191 & 0.271 & $\mathrm{CD} 4+/ \mathrm{CD} 8+\mathrm{T}$ cell ratio & 0.140 & 0.384 \\
\hline CD56+ NK cells, $\%$ of total cells & 0.161 & 0.355 & CD56+ NK cells, $\%$ of total cells & 0.072 & 0.649 \\
\hline CD11+ M1 macrophages, \% of total cells & 0.239 & 0.154 & CD11+ M1 macrophages, \% of total cells & 0.534 & $<0.001$ \\
\hline CD11- M2 macrophages, \% of total cells & 0.177 & 0.295 & CD11- M2 macrophages, \% of total cells & 0.425 & 0.005 \\
\hline M1/M2 ratio & 0.013 & 0.940 & $\mathrm{M} 1 / \mathrm{M} 2$ ratio & 0.098 & 0.536 \\
\hline CD19+ B lymphocytes, \% of total cells & 0.111 & 0.526 & CD19+ B lymphocytes, \% of total cells & 0.357 & 0.020 \\
\hline Subcutaneous basal lipolysis (per cell) & $\mathbf{R}$ & p value & Visceral basal lipolysis (per cell) & $\mathbf{R}$ & p value \\
\hline Total CD45+ leukocytes, \% of total cells & -0.163 & 0.348 & Total CD45+ leukocytes, \% of total cells & 0.338 & 0.044 \\
\hline CD3+ $\mathrm{T}$ cells, $\%$ of total cells & 0.077 & 0.665 & CD3+ T cells, $\%$ of total cells & 0.091 & 0.597 \\
\hline CD3+CD4+ T-helper cells, \% of total cells & 0.043 & 0.809 & CD3+CD4+ T-helper cells, \% of total cells & 0.181 & 0.292 \\
\hline CD3+CD8+ cytotoxic T cells, \% of total cells & -0.072 & 0.687 & CD3+CD8+ cytotoxic T cells, \% of total cells & 0.071 & 0.684 \\
\hline $\mathrm{CD} 4+/ \mathrm{CD} 8+\mathrm{T}$ cell ratio & 0.245 & 0.163 & $\mathrm{CD} 4+/ \mathrm{CD} 8+\mathrm{T}$ cell ratio & 0.182 & 0.296 \\
\hline CD56+ NK cells, $\%$ of total cells & 0.223 & 0.204 & CD56+ NK cells, \% of total cells & 0.193 & 0.260 \\
\hline CD11+ M1 macrophages, \% of total cells & -0.024 & 0.893 & CD11+ M1 macrophages, \% of total cells & 0.261 & 0.125 \\
\hline CD11- M2 macrophages, \% of total cells & -0.044 & 0.800 & CD11- M2 macrophages, \% of total cells & 0.152 & 0.168 \\
\hline $\mathrm{M} 1 / \mathrm{M} 2$ ratio & -0.014 & 0.935 & $\mathrm{M} 1 / \mathrm{M} 2$ ratio & -0.032 & 0.853 \\
\hline CD19+ B lymphocytes, \% of total cells & 0.196 & 0.267 & CD19+ B lymphocytes, \% of total cells & -0.179 & 0.296 \\
\hline
\end{tabular}






\section{Chapter 5}

Coordinated regulation of adipose tissue adrenergic- and non-adrenergicmediated lipolysis during exercise in

lean and obese individuals: the effect of exercise training

Kenneth Verboven, Rudi Stinkens, Dominique Hansen, Inez Wens, Ines Frederix, Bert Op 't Eijnde, Johan WE Jocken,

Gijs H Goossens, Ellen E Blaak 


\section{Abstract}

Aims/hypothesis: Adipose tissue dysfunction, which includes impairments in (adipose tissue) lipolysis, contributes to insulin resistance. Subcutaneous adipose tissue (SCAT) lipolysis in obesity is characterized by catecholamine resistance and an impaired ANP responsiveness. It remains to be established whether exercise training improves (non-)adrenergically-mediated lipolysis in metabolically compromised conditions. The aim of the present study was to investigate the effect of local combined $a$ - and $\beta$-adrenergic receptor blockade on SCAT lipolysis in obese insulin sensitive (IS), obese insulin resistant (IR) and age-matched lean IS men. Obese individuals underwent exercise training to improve metabolic profile and (non)adrenergically-mediated SCAT lipolysis. Methods: Abdominal SCAT lipolysis was investigated in 10 obese IS, 10 obese IR and 10 age-matched lean IS men using microdialysis in the presence or absence of local combined $a$ - and $\beta$-adrenergic receptor blockade at rest, during 60 min low-intense $\left(40 \% V^{2} O_{2 \max }\right)$ endurance-type exercise and recovery. Systemic responses were investigated using venous blood sampling. Obese individuals participated in a supervised, endurance and resistance exercise training intervention for 12 weeks (3 sessions/week) after which the microdialysis measurements were repeated in obese IR men.

Results: Exercise-induced increase in SCAT lipolysis (expressed as total area under the curve) was more pronounced in obese IS (81\%) and IR (34\%) as compared to lean individuals $\left(P_{\text {group }}=0.012\right)$. SCAT lipolysis was significantly reduced $(\sim 40 \%)$ following local combined $a-/ \beta$-adrenoceptor blockade in obese IS individuals only. Despite improvements in body composition, physical fitness and exercise-induced changes in circulating FFA, lactate and adrenalin, exercise intervention did not significantly affect (non-)adrenergically-mediated lipolysis in abdominal SCAT of obese IR individuals.

Conclusion: Our findings indicate a major contribution of non-adrenergically mediated lipolysis during exercise in abdominal SCAT of lean and obese individuals. Furthermore, the exercise training program improved metabolic profile and body composition in obese individuals, but did not affect abdominal SCAT lipolysis.

ClinicalTrial.gov accession number NCT02418728 


\section{Introduction}

Adipose tissue (AT) dysfunction is commonly observed in human obesity and contributes to insulin resistance (IR) and chronic metabolic diseases, including cardiovascular disease, type 2 diabetes mellitus (T2D) and certain types of cancer 1,2. Disturbances in AT lipid metabolism, including a decreased lipid uptake and impairments in lipid mobilization are closely linked to ectopic fat deposition and obesity-related IR ${ }^{3}$. An important function of the AT is to release fatty acids through lipolysis ${ }^{4,5}$, especially during fasting and increased energy demanding conditions such as exercise. Multiple endocrine factors affect the activity of lipid droplet-associated proteins and lipases, thereby regulating the release of free fatty acids (FFA) and glycerol ${ }^{6}$. However, impairments in the regulation of lipolysis have been identified in subcutaneous AT (SCAT) of obese humans 7 , including a blunted catecholamine-mediated lipolysis 8,9. More specific, $\beta$-adrenergically-mediated lipolysis is reduced 9 and inhibitory $a_{2}-$ adrenoceptors become predominant on adipocytes in the obese insulin resistant state ${ }^{10,11}$, leading to a blunted adrenergically-mediated lipolysis ${ }^{9,12}$. Of interest, local $\beta$-adrenergic blockade (alone or in combination with $a_{2}$-adrenergic blockade) in SCAT, inhibits exercise-induced lipolysis only to a minor extent at low-to-moderate intensities in healthy lean ${ }^{13-15}$ and overweight individuals ${ }^{16}$. In this respect, Moro et al. ${ }^{16}$ demonstrated that non-adrenergically-mediated lipolysis in SCAT substantially contributes to lipid mobilization during exercise in healthy young lean men ${ }^{15}$ and healthy young overweight men ${ }^{16}$. Other key regulators of lipolysis are insulin ${ }^{17}$ and lactate ${ }^{18}$, which both exert an inhibitory role in the physiological control of AT during exercise ${ }^{17-19}$.

More recently, evidence has emerged that natriuretic peptides (NP) not only affect the cardiovascular system, but also have pronounced effects in several key metabolic organs such as AT and skeletal muscle ${ }^{20}$. Interestingly, several studies have indicated that the circulating NP concentrations are reduced in human obesity and T2D ${ }^{21-23}$. The latter findings, together with evidence that reduced systemic NP concentrations increase the risk of developing T2D 24,25, highlight the importance of NP in metabolic disease. Of the NP family, atrial natriuretic peptide (ANP) has been shown to be the most potent stimulator of human AT lipolysis ${ }^{26}$, via guanylyl cyclase-coupled natriuretic peptide receptor 
type A (NPRA)-mediated activation of hormone-sensitive lipase (HSL) 27,28. Interestingly, we have recently found that maximal ANP responsiveness is impaired in isolated abdominal subcutaneous adipocytes of obese non-diabetic and T2D men ${ }^{29}$. In line, Rydén and colleagues ${ }^{30}$ have recently shown a blunted lipolytic effect of ANP in isolated abdominal subcutaneous adipocytes of obese women and in situ (microdialysis) in abdominal SCAT of overweight men under resting conditions. Importantly, however, the physiological role of exerciseinduced ANP-mediated lipolysis in human obesity remains to be established.

It has been shown that endurance exercise training can partly improve $\beta$ adrenoceptor activity, reduce anti-lipolytic $a_{2}$-adrenoceptor sensitivity in human SCAT ${ }^{31-33}$, and alleviate ANP-mediated lipolysis in subcutaneous adipocytes in young, metabolically healthy overweight individuals 28,34 . However, to date, it remains elusive if endurance and resistance exercise intervention improves ANPinduced activation of lipolysis in metabolically compromised conditions.

The aim of the present study was to compare the effect of local combined a- and $\beta$-adrenoceptor blockade on local SCAT lipolysis at rest, during low-intensity endurance-type exercise and during recovery from exercise in middle-aged obese insulin sensitive (IS), obese insulin resistant (IR) and age-matched lean IS men. In addition, we investigated whether a 12-week endurance and resistance exercise training improved the metabolic profile in obese men and (non-)adrenergically-mediated abdominal SCAT lipolysis in obese IR men. 


\section{Methods}

Subjects - Ten middle-aged healthy lean insulin sensitive (IS), 10 obese IS and 10 obese insulin resistant (IR) men, matched for age and BMI (obese groups) participated in the present study. Subjects were included when they had a stable body weight for at least 3 months prior to the start of the intervention and had no contraindications for participation in an exercise training intervention based on their medical history. Major exclusion criteria were a history, or clinical symptoms, of heart, lung or kidney disease, presence of endocrine anomalies and/or the use of beta-blockers, glucose or lipid-lowering medication. Insulin sensitivity was assessed via homeostasis assessment of insulin resistance (HOMA-IR) ${ }^{35}$. Subjects were classified as insulin sensitive or insulin resistant when HOMA-IR was $\leq 2.3^{36}$ or $\geq 3.8^{37}$, respectively. Height, weight, waist and hip circumference and blood pressure were measured during screening. Body composition was measured using a Dual Energy X-ray Absorptiometry scan (Hologic Series Delphi-A Fan Beam X-ray Bone Densitometer). One week before the investigational protocol, peak oxygen uptake $\left(\mathrm{VO}_{2 \text { peak }}\right)$ was determined during a maximal cardiopulmonary exercise test performed on an electrical braked cycle ergometer (Gymna Ergofit Cycle 400, Bilzen, Belgium) by using an incremental procedure (work rate increased by $15 \mathrm{~W} / \mathrm{min}$ until volitional exhaustion). Heart rate (electrocardiography) was monitored continuously and $\mathrm{VO}_{2 \text { peak }}$ was measured using a Metalyzer II (Cortex Medical, Leipzig, Germany). The study was approved by the Medical Ethical Committee of the Jessa Hospital and Hasselt University, Hasselt, Belgium, and performed in accordance with the declaration of Helsinki (2008). All individuals gave written informed consent prior to the start of the study.

Experimental protocol - Subjects arrived at the hospital at 07:30 AM after an overnight fast. They were instructed to consume a standardized meal and snack the evening before the test day (total energy: $2628 \mathrm{~kJ}$ (626 kcal); $23.4 \mathrm{~g}$ fat ( $10.4 \mathrm{~g}$ saturated fat); $73.8 \mathrm{~g}$ carbohydrates (of which $6.8 \mathrm{~g}$ sugar); $28.8 \mathrm{~g}$ protein; $2.9 \mathrm{~g}$ salt; $2.3 \mathrm{~g}$ fibres) and to abstain from exhausting activities 48 hours prior to the experimental protocol. On arrival, a catheter was inserted into the antecubital vein for blood sampling. Two microdialysis catheters (CMA 63, CMA Microdialysis AB, Stockholm, Sweden) were inserted percutaneously into 
SCAT after epidermal anesthesia (EMLA ${ }^{\circledR}$ crème: lidocaine $2.5 \%$ and prilocaine $2.5 \%$, AstraZeneca $A B$ ) at a distance of $6-8 \mathrm{~cm}$ from the umbilicus (one probe on the left side and one probe on the right side of the umbilicus). The probes were connected to a microinfusion pump (Harvard apparatus, Plato BV, Diemen, The Netherlands) and perfused with Ringer solution (in mmol/l: 147 sodium, 4 potassium, 2.25 calcium and 156 chloride; Fresenius Kabi BV, 's Hertogenbosch, The Netherlands) at a perfusion rate of $2.0 \mu \mathrm{l} / \mathrm{min}$. Ethanol $(50 \mathrm{mmol} / \mathrm{l}$ ) was added to the perfusate to semi-qualitatively estimate changes in local adipose tissue blood flow (ATBF), using the ethanol outflow/inflow (out/in) ratio ${ }^{38}$. A higher ethanol out/in ratio, corresponding to a lower ethanol wash-out, reflects a lower regional ATBF.

One microdialysis catheter was perfused with Ringer solution (control), while the contralateral catheter was perfused with Ringer, supplemented with $100 \mu \mathrm{mol} / \mathrm{I}$ phentolamine ( $a_{1,2}$-adrenergic receptor antagonist) (Regitin $10 \mathrm{mg} / \mathrm{ml}$; Novartis Pharma BV, The Netherlands) and $100 \mu \mathrm{mol} / \mathrm{I}$ propranolol (nonselective $\beta$ adrenergic receptor antagonist) (propranolol hydrochloride, Dociton $1 \mathrm{mg} / \mathrm{ml}$, Mibe $\mathrm{GmbH}$, Germany), concentrations that completely suppress lipolysis $28,39,40$. After a 60-min equilibration period (recovery from insertion), two 30-min fraction of dialysate were collected at a flow rate of $0.3 \mu \mathrm{L} / \mathrm{min}$ after which the perfusion rate was increased to $2.0 \mu \mathrm{L} / \mathrm{min}$ for the remaining of the experiment. During the resting phase, three 15 -min fractions of the outgoing dialysate were collected from both sites to determine the extracellular glycerol concentration (reflecting basal lipolysis). Next, subjects performed a single bout of endurance exercise for $60 \mathrm{~min}$ at $40 \%$ of their $\mathrm{VO}_{2 \max }$ on a cycle ergometer while heart rate was monitored continuously (Polar, Kempele, Finland). Exercise was followed by a 60-min recovery period in supine position. During exercise and recovery, dialysate samples were collected at 15 min intervals without disconnecting the microdialysis probes from the microinfusion pumps.

Ethanol concentrations were determined both in the ingoing (perfusate) and outgoing (dialysate) fluid to assess the ethanol out/in ratio as an indicator of local nutritive blood flow. Ethanol concentrations were determined at the same day, whereas dialysate samples for measurement of extracellular glycerol, 
glucose and lactate concentrations were immediately frozen and stored at -80 ${ }^{\circ} \mathrm{C}$ until analysis. Venous blood samples were taken at rest, during exercise and recovery in prechilled $20 \mathrm{~mL}$ tubes at 15 min intervals throughout the study protocol.

Indirect calorimetry - Substrate utilization and energy expenditure were determined at rest and during submaximal exercise via indirect calorimetry using a Metalyzer II (Cortex Medical, Leipzig, Germany). Substrate oxidation rates $(\mathrm{g} / \mathrm{min})$ and energy expenditure were calculated from $\mathrm{VO}_{2}$ and $\mathrm{VCO}_{2}{ }^{41,42}$. Water intake was allowed ad libitum during the exercise and recovery period.

Exercise training protocol - Obese IS and IR subjects participated in a supervised, exercise training program for 12 weeks ( 3 sessions per week) ${ }^{43}$. Subjects were asked not to change their habitual diet during the intervention period. Each training session started with cycling (Excite Bike, Technogym, Zaventem, Belgium) for $45 \mathrm{~min}$ at $65 \% \mathrm{VO}_{2 \text { peak }}$ (heart rate based) from which mean heart rate, mean workload and total energy expenditure (calories) were collected. Next, resistance exercises of 5 large muscle groups were performed at $65-70 \%$ of 1 RM (leg press, leg curl, leg extension, vertical traction, arm curl and chest press; Technogym). Training volume and load were gradually increased during the intervention whereby resistance training was increased every 3 weeks. Training sessions were supervised to assure compliance and safety of the participants. After the 12 weeks of exercise training the experimental protocol was repeated and venous blood samples were taken at rest, during exercise and recovery at 15 min intervals. In addition, SCAT microdialysis was performed in obese IR subjects, as described above.

Biochemical analysis - Microdialysate samples were analysed for glycerol, glucose and lactate concentrations by means of bioluminescence on an ISCUS clinical microdialysis analyser ( $M$ dialysis $A B$, Stockholm, Sweden). Ethanol concentrations in dialysate (out) and perfusate (in) were measured spectrophotometrically using a COBAS FARA semi-automatic analyser (Roche Diagnostics, Basal, Switzerland) and using a standard ethanol assay kit (Boehringer Mannheim, Germany). 
Blood samples were centrifuged at $4^{\circ} \mathrm{C}$ for $10 \mathrm{~min}$ at $1200 \mathrm{~g}$ and plasma and serum was stored at $-80^{\circ} \mathrm{C}$ until further analysis. Plasma free glycerol was measured after precipitation with an enzymatic assay (Enzytec ${ }^{\mathrm{TM}}$ Glycerol, Roche Biopharm, Switzerland), automated on a Cobas Fara spectrophotometric autoanalyser (Roche Diagnostics, Basel, Switzerland). Plasma FFA, glucose and lactate concentrations were measured with enzymatic assays on an automated spectrophotometer (ABX Pentra 400 autoanalyser, Horiba ABX, Montpellier, France). Plasma ANP concentrations were measured using an enzyme immunoassay (RayBiotech, Norcross GA, USA). Catecholamine concentrations (adrenalin and noradrenalin) were determined using high performance liquid chromatography with electrochemical detection (ClinRep ${ }^{\circledR}$ Complete Kit for Catecholamines in Plasma, RECIPE chemicals \& Instruments $\mathrm{GmbH}$, Munich, Germany). Serum insulin concentrations were determined with radioimmunoassay kits (Human Insulin specific RIA Kit, Millipore Corporation, MA, USA).

Statistical analysis - All data are expressed as means \pm SEM. Normal distribution was tested by the Kolmogorov-Smirnov test. Subjects were excluded from analyses when dialysate samples of 2 subsequent time points were missing, in order to maintain paired samples. Dialysate and systemic exercise responses were expressed as the area under the curve (AUC) and the incremental area under the curve (iAUC), calculated by the trapezoid method. Cross-sectional analyses (differences between groups and conditions) for the microdialysis lipolysis data were analysed with a two-way repeated-measures ANOVA. In case of significance, post-hoc analyses with Bonferroni correction were applied to identify significant within-group effects. Differences in plasma concentrations and substrate metabolism between groups were tested with a one-way ANOVA and differences within groups were analysed by means of paired $t$-test. Intervention effects in the obese groups were analysed with a twoway repeated-measures ANOVA (with pre- and post-intervention as conditions), with Bonferroni post-hoc correction to detect within-group effects. Three subjects dropped out of the exercise intervention, due to medical $(n=1)$ or motivational reasons $(n=2)$ and were therefore excluded from the intervention 
(pre vs. post) analyses. SPSS 21 for Macintosh OS $X$ was used to perform all calculations (IBM Corporation, Armonk, NY, USA). The level of statistical significance was set at $p<0.05$ ( 2 -tailed), while $p<0.10$ was considered a tendency. 


\section{Results}

\section{Baseline}

\section{Anthropometric and clinical characteristics}

Subjects' characteristics are presented in Table 1. By design, there was a significant difference between the lean and the obese IS and/or obese IR group with respect to body weight, BMI, WH-ratio, whole-body fat percentage, android (i.e. trunk region) and gynoid (including hip and leg regions) fat mass (all $\mathrm{p}<0.05)$. Furthermore, HOMA-IR and fasting serum insulin concentrations were significantly higher in obese IR compared to the lean and obese IS individuals ( $p<0.001$ for both parameters in both groups). Obese IS and obese IR individuals only differed in android fat mass, which was higher in the obese IR group $(p=0.017)$ (Table 1 ). With respect to physical fitness, $V_{2 \text { peak/FFM }}$ and $\mathrm{W}_{\text {peak/FFM }}$ were significantly lower in the obese IS and obese IR group as compared to the lean group ( $p<0.01$ and $p<0.001$, respectively) (Table 1 ), while maximal heart rate and maximal respiratory quotient (RQ) were comparable between groups.

Table 1 Characteristics of obese insulin sensitive, obese insulin resistant and healthy lean controls

\begin{tabular}{ccccc} 
& Lean & $\begin{array}{c}\text { Obese insulin } \\
\text { sensitive } \\
(n=10)\end{array}$ & $\begin{array}{c}\text { Obese insulin } \\
\text { resistant } \\
(n=10)\end{array}$ & PNOVA \\
\hline Age, years & $45 \pm 2$ & $47 \pm 2$ & $43 \pm 1$ & 0.527
\end{tabular}

\section{Cardiometabolic risk}

Fasting plasma glucose ( $\mathrm{mmol} / \mathrm{L})$

Fasting serum insulin $(\mathrm{mU} / \mathrm{L})$

HOMA-IR

Systolic BP $(\mathrm{mmHg})$

Diastolic BP $(\mathrm{mmHg})$

\section{Body composition}

Body weight $(\mathrm{kg})$

Body mass index $\left(\mathrm{kg} / \mathrm{m}^{2}\right)$

Waist-to-hip ratio

Fat mass $(\mathrm{kg})$

Fat percentage (\%)

Fat free mass $(\mathrm{kg})$

$\begin{array}{cc}5.5 \pm 0.0 & 5.2 \pm 0.1 \\ 7.3 \pm 0.6 & 9.2 \pm 0.6 \\ 1.8 \pm 0.1 & 2.1 \pm 0.1 \\ 122 \pm 2 & 135 \pm 6 \\ 72 \pm 1 & 81 \pm 4\end{array}$

$79.9 \pm 2.9$

$23.7 \pm 0.4$

$1.00 \pm 0.00$

$16.4 \pm 1.1$

$22.0 \pm 0.8$

$57.4 \pm 1.8$

$62 \pm 3$

$4.9 \pm 0.1$

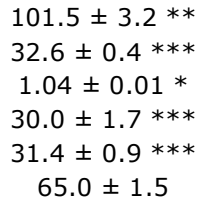

$5.8 \pm 0.2+$

$19.6 \pm 1.6 * * *+$

$5.0 \pm 0.4 * * *+$

$143 \pm 6 *$

$86 \pm 5$

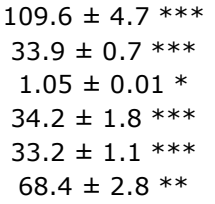

$<0.001$

$<0.001$

0.010

$<0.001$

$<0.001$

0.004

\section{Exercise capacity}

$\mathrm{VO}_{\text {peak }}\left(\mathrm{ml}^{*} \mathrm{~min}^{-1} * \mathrm{~kg}^{-1}(\mathrm{FFM})\right)$

$\mathrm{W}_{\text {peak }}\left(\right.$ Watt* $\mathrm{kg}^{-1}$ (FFM))

$48 \pm 1 * *$

0.002

$3.4 \pm 0.1 * * *$ $<0.001$

Data are mean \pm SE. * Significantly different from lean group $\mathrm{p}<0.05 ; * * \mathrm{p}<0.01 ; * * * \mathrm{p}<0.001$

+ Significantly different from obese insulin sensitive group $(p<0,05)$. FFM: fat free mass; HR: heart rate; RER: respiratory exchange ratio; $\mathrm{VO}_{2}$ peak: maximum oxygen uptake; Wmax: maximum power output 
Under resting conditions, plasma glycerol, FFA, glucose, lactate, ANP, adrenalin and noradrenalin concentrations were comparable between groups, while fasting serum insulin concentration was higher in obese IR compared to lean and obese IS individuals (Figure $1 \mathrm{~A}-\mathrm{H}$ ). During exercise, plasma concentrations of glycerol, FFA, glucose, ANP, adrenalin and noradrenalin increased to the same extent in all groups (Figure 1). Exercise increased plasma lactate concentrations in all groups, which was most pronounced in obese IR as compared to lean individuals $\left(p_{\text {ANOVA }}=0.044\right)$ (Figure $\left.1 \mathrm{D}\right)$. The exercise-induced increase in plasma ANP was similar in all groups $\left(\mathrm{p}_{\mathrm{ANOVA}}=0.300\right)$, but peak plasma ANP concentrations were reached earlier during exercise in the lean compared to the obese groups $(p=0.034)$ (Figure $1 \mathrm{E})$. Serum insulin levels were significantly higher during exercise in obese IR as compared to obese IS and lean individuals, with no differences between the latter two groups (Figure $1 \mathrm{~F}$ ). During exercise, the increase in plasma adrenalin and noradrenalin concentrations was comparable between groups (Figure $1 \mathrm{G}-\mathrm{H}$ ). During recovery, plasma glycerol, glucose, adrenalin, noradrenalin and ANP concentrations decreased back to baseline concentrations (Figure 1), while plasma lactate concentrations tended to remain elevated in the obese IR group ( $p_{A N O V A}=0.061$ ) (Figure $\left.1 \mathrm{D}\right)$. Furthermore, plasma FFA concentrations peaked in the first 15 minutes of the recovery period and remained significantly elevated in obese IR as compared to lean individuals $\left(p_{\text {ANOVA }}=0.020\right)$ (Figure $1 \mathrm{~B}$ ). Serum insulin concentrations remained significantly elevated during recovery in the obese IR group compared to the obese IS and lean group (both $\mathrm{p}_{\mathrm{ANOVA}}<0.001$ ), with no differences between the latter two groups (Figure $1 \mathrm{~F}$ ). Detailed systemic plasma responses during baseline, exercise and recovery are shown in Supplemental Table S1. 
A

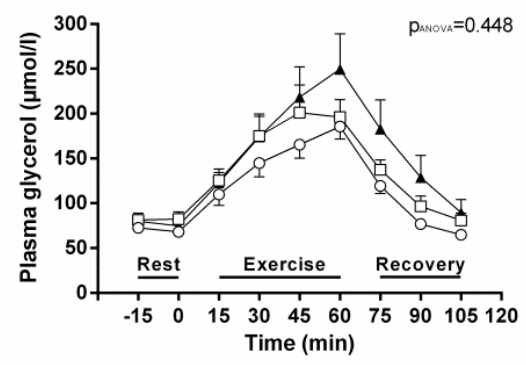

C

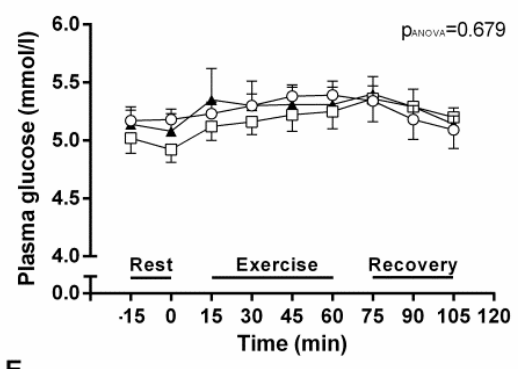

E

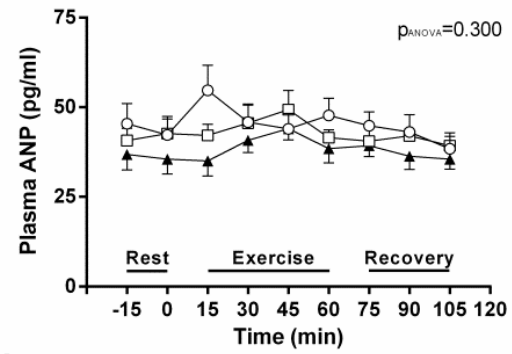

G

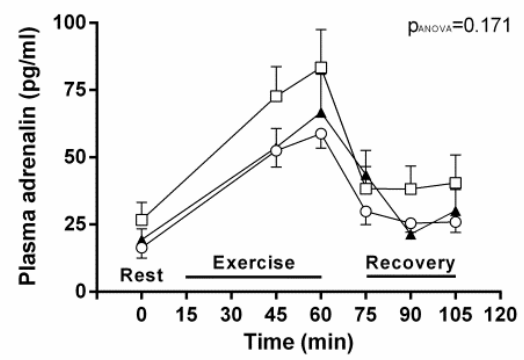

B

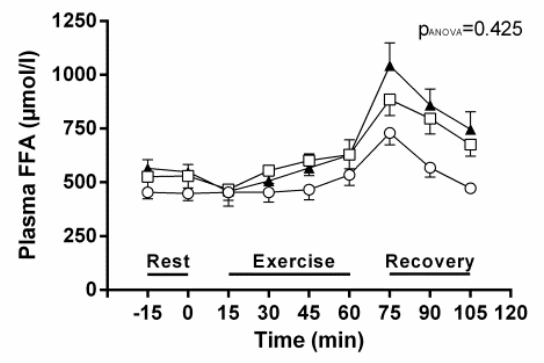

D

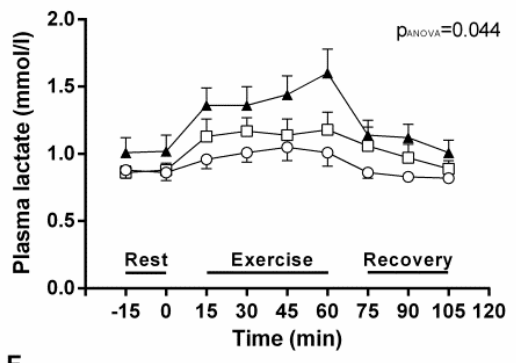

F

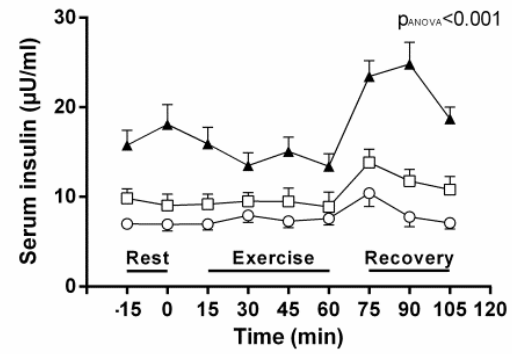

$\mathrm{H}$

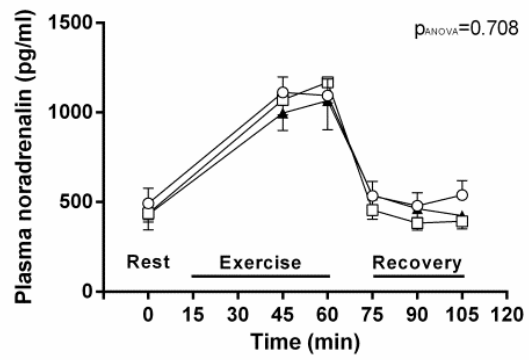

Figure 1 - Plasma glycerol, FFA, glucose, lactate, ANP, adrenalin, noradrenalin and serum insulin concentrations at rest, during exercise and recovery. Systemic glycerol (A), FFA (B), glucose (C), lactate (D), ANP (E), insulin (F), adrenalin (G) and noradrenalin (H) responses in lean (white circles), obese insulin sensitive (white squares) and obese insulin resistant (black triangles) individuals. Data are presented as mean \pm SEM. $P_{\text {ANOVA }}$ values represent differences in exercise-induced systemic responses between groups. 
Substrate oxidation and energy expenditure were determined at rest and during exercise. Whole-body energy expenditure $(\mathrm{kJ} / \mathrm{min}), \mathrm{RQ}$, carbohydrate and fat oxidation (as percentage of energy expenditure) were not different between groups at rest, nor during exercise (Supplemental Figure $1 \mathrm{~A}-\mathrm{E}$ ).

\section{Microdialysis}

Abdominal subcutaneous adipose tissue blood flow

At rest, lean individuals had a significantly lower ethanol out/in ratio, reflecting a higher ATBF, compared to both obese groups $(p<0.01)$, whilst no significant difference in ATBF was observed between both obese groups (Figure $2 \mathrm{~A}, \mathrm{C}$ and $E)$. Local $a-/ \beta$-adrenergic blockade induced a significant increase in ethanol out/in ratio in the lean group (Figure $2 \mathrm{~A}$ ), reflecting a reduced ATBF, while this effect disappeared during exercise. Moreover, this adrenergic sensitivity of ATBF was not observed in the obese IS or IR group (Figure $2 \mathrm{C}$ and $\mathrm{E}$ ). Exercise induced a decrease in ethanol out/in ratio, reflecting an increase in ATBF, in all groups. This exercise-induced increase in ATBF tended to be higher in lean as compared to obese IS and obese IR individuals $(p=0.093, p=0.087$, respectively) (Figure $2 \mathrm{~A}, \mathrm{C}$ and $\mathrm{E}$ ). During recovery, ATBF returned to resting levels, with a significantly higher ATBF (i.e. a lower ethanol out/in ratio) in the lean group compared to both obese groups (Figure 2 A, C and E). Details with respect to ethanol out/in ratio during baseline, exercise and recovery are shown in Supplemental Table S2. 
A
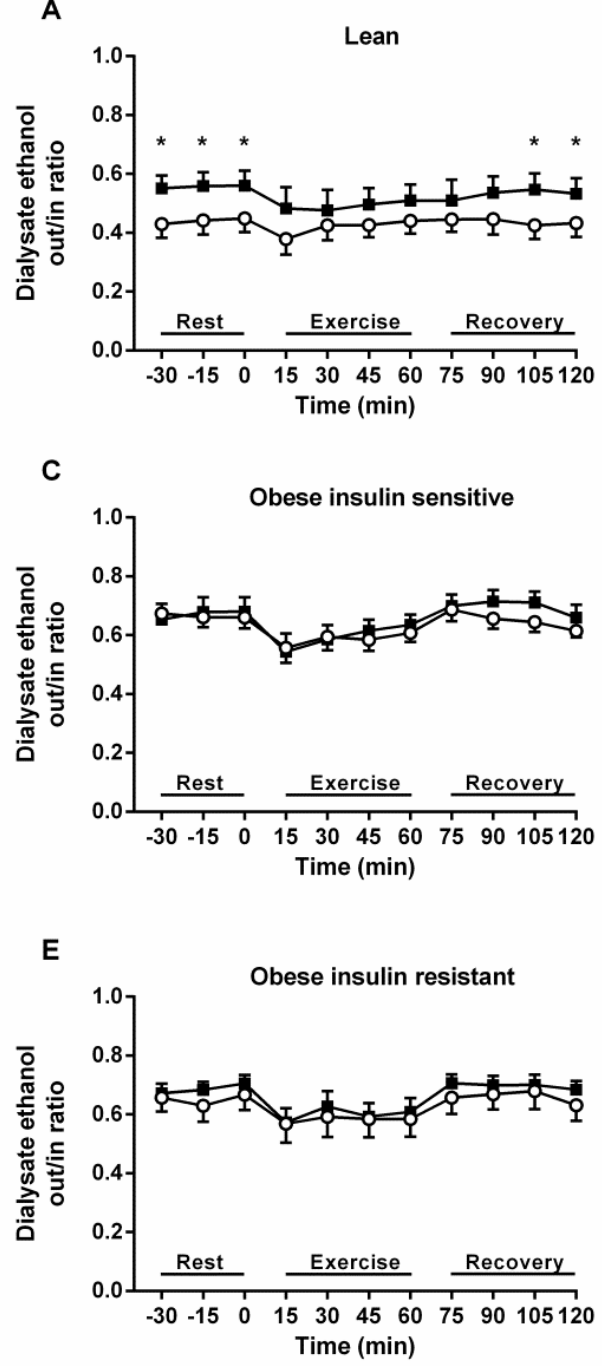

B
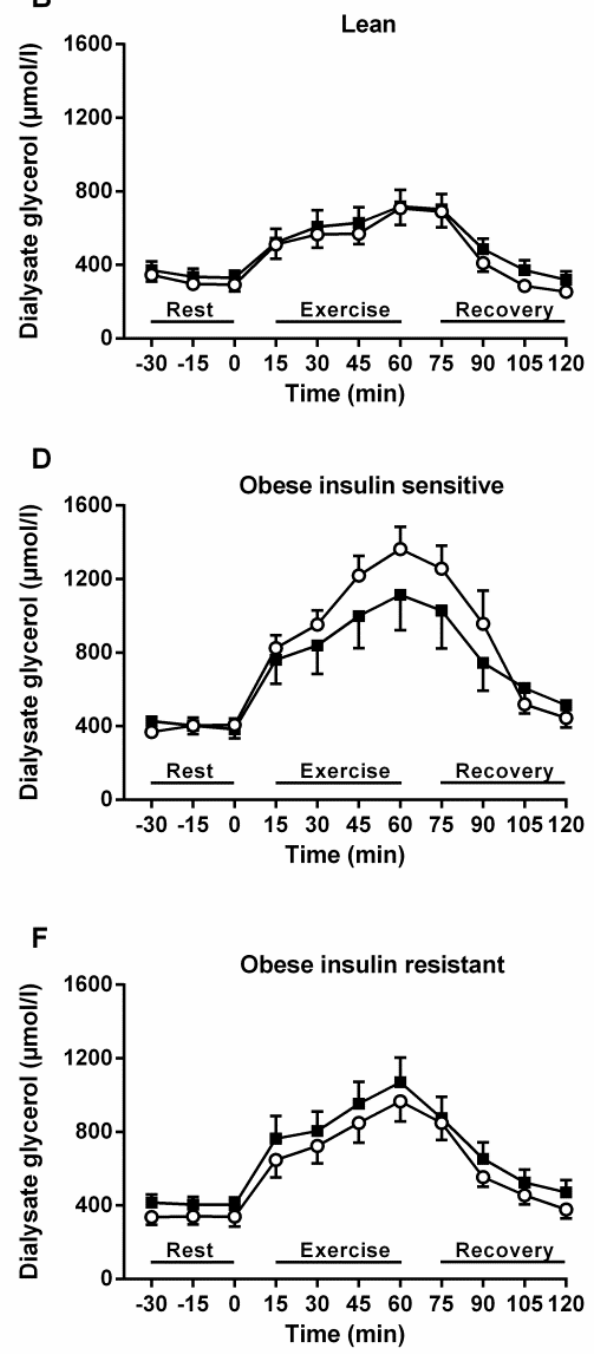

Figure 2 - Changes in subcutaneous adipose tissue extracellular glycerol concentration and adipose tissue blood flow indices. Subcutaneous adipose tissue ethanol ratio's in lean (A), obese insulin sensitive (B) and obese insulin resistant (C) individuals. Changes in extracellular glycerol concentration in lean (D), obese insulin sensitive (E) and obese insulin resistant (F) individuals at rest, during exercise and recovery in control probe (white circles) and the probe perfused with phentolamine and propranolol (black squares). Data are presented as mean \pm SEM. * Significantly different from the control probe $(p<0.05)$. 
In SCAT, resting extracellular glycerol concentrations were comparable between groups (Figure $2 \mathrm{~B}, \mathrm{D}$ and $\mathrm{F}$ ). Local $\mathrm{a}-/ \beta$-adrenergic blockade had no significant effects on resting extracellular SCAT glycerol concentration in either of the groups (Figure $2 \mathrm{~B}, \mathrm{D}$ and F). During exercise, extracellular glycerol concentration significantly increased in all groups. In obese IS individuals, exercise-induced increase in glycerol concentration $\left(\mathrm{AUC}_{0-60}\right)$ was higher compared to lean $(p=0.011)$, but not obese IR individuals $(p=0.816)$ (Figure 2 $B, D$ and $F$ ). Local $a-/ \beta$-adrenergic blockade induced a significant reduction in the exercise-induced increase in extracellular glycerol in the obese IS group $(p=0.020)$, but not in the lean IS or obese IR group (Figure 3$)$. During recovery, extracellular glycerol concentrations decreased in all groups, but remained significantly elevated in the obese IS as compared to the lean group, with no differences between both obese groups (Figure 2 B, D and F). Additionally, there were no significant effects of $\alpha-\beta$-adrenergic blockade on the extracellular glycerol concentration during recovery in any group (Figure $2 \mathrm{~B}, \mathrm{D}$ and $\mathrm{F}$ ). Details with respect to dialysate glycerol concentrations at rest, during exercise and recovery are shown in Supplemental Table S2. Abdominal SCAT extracellular glucose and lactate concentrations and responses were comparable between groups at rest, during exercise and recovery (Supplemental Table S3). 


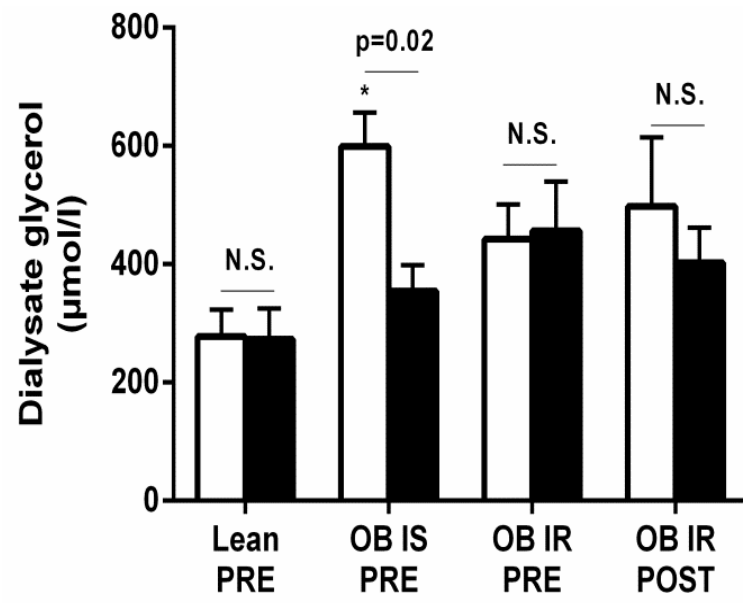

Figure 3 - Exercise-induced increase in subcutaneous adipose tissue extracellular glycerol concentration. Mean changes in subcutaneous adipose tissue extracellular glycerol concentration during $1 \mathrm{~h}$ of low-intense exercise ( $40 \% \mathrm{VO}_{2 \text { peak }}$ ). Extracellular glycerol concentrations are determined in control probe (white bars) and the probe perfused with phentolamine and propranolol (black bars). Changes were calculated by the difference between the mean glycerol concentrations during exercise and the baseline concentration ( $p_{\text {group }}=0.009, p_{\text {treatment }}=0.069, p_{\text {treatment*group }}=0.035$ ). Data are presented as mean \pm SEM for pre-intervention (PRE) and post-intervention (POST) measurement. * Significantly $(p<0.05)$ different compared to the control probe from the lean group; N.S.: not significant.

\section{Exercise training intervention}

Anthropometry, exercise capacity and systemic responses

In both obese groups, exercise training led to a significant reduction in body weight, BMI, whole-body fat percentage as well as android and gynoid fat mass (Table 2). Whole-body insulin sensitivity (HOMA-IR) was significantly improved in the obese IR group ( $\left.p_{\text {time }}=0.005\right)$, but not in the obese IS group. Furthermore, physical fitness $\left(\mathrm{VO}_{2 \text { peak/FFM }}\right.$ as well as $\left.\mathrm{W}_{\text {max/FFM }}\right)$ improved significantly following the 12 -week exercise training (Table 2 ). 
Body composition and exercise capacity before and after 12 weeks of endurance and resistance training

\begin{tabular}{|c|c|c|c|c|c|c|c|}
\hline \multirow[b]{2}{*}{ Body composition } & \multicolumn{2}{|c|}{ Obese insulin sensitive $(n=8)$} & \multicolumn{2}{|c|}{ Obese insulin resistant $(n=9)$} & \multirow[b]{2}{*}{ P Time } & \multirow[b]{2}{*}{ P Group } & \multirow[b]{2}{*}{ P Time*Group } \\
\hline & Pre & Post & Pre & Post & & & \\
\hline Body weight (kg) & $104.5 \pm 3.2$ & $102.8 \pm 2.6$ & $110.6 \pm 5.1$ & $108.3 \pm 5.0$ & 0.002 & 0.360 & 0.585 \\
\hline Body mass index $\left(\mathrm{kg} / \mathrm{m}^{2}\right)$ & $32.7 \pm 0.5$ & $32.2 \pm 0.3$ & $34.1 \pm 0.8$ & $33.3 \pm 0.8$ & 0.002 & 0.225 & 0.550 \\
\hline Waist circumference $(\mathrm{cm})$ & $108.8 \pm 1.6$ & $108.8 \pm 1.5$ & $117.6 \pm 3.3$ & $113.5 \pm 1.3$ & 0.039 & 0.004 & 0.076 \\
\hline Fat mass $(\mathrm{kg})$ & $31.22 \pm 1.91$ & $29.11 \pm 1.69$ & $35.00 \pm 1.92$ & $33.07 \pm 2.02$ & $<0.001$ & 0.168 & 0.833 \\
\hline Fat percentage (\%) & $31.6 \pm 1.1$ & $30.0 \pm 1.0$ & $33.7 \pm 1.1$ & $32.8 \pm 1.2$ & $<0.001$ & 0.150 & 0.267 \\
\hline Fat free mass (kg) & $66.8 \pm 1.4$ & $67.2 \pm 1.3$ & $68.5 \pm 3.1$ & $67.5 \pm 3.3$ & 0.377 & 0.779 & 0.037 \\
\hline \multicolumn{8}{|l|}{ Exercise capacity } \\
\hline $\mathrm{VO} 2_{\text {peak }}\left(\mathrm{ml} * \mathrm{~min}^{-1} * \mathrm{~kg}^{-1}(\mathrm{FFM})\right)$ & $49 \pm 2$ & $53 \pm 1$ & $48 \pm 1$ & $55 \pm 2$ & 0.012 & 0.800 & 0.521 \\
\hline $\mathrm{W}_{\text {peak }}\left(\mathrm{Watt}^{*} \mathrm{~kg}^{-1}(\mathrm{FFM})\right)$ & $3.8 \pm 0.2$ & $4.1 \pm 0.1$ & $3.4 \pm 0.1$ & $4.0 \pm 0.1$ & $<0.001$ & 0.382 & 0.069 \\
\hline
\end{tabular}

Data are mean \pm SE. FFM: fat free mass; VO2 peak: maximum oxygen uptake; Wmax: maximum power output 
The training intervention induced a significant reduction in resting plasma FFA (Figure $4 \mathrm{C}$ and $\mathrm{D}$ ) and tended to reduce fasting ANP concentrations in both obese groups (Figure $4 \mathrm{~K}$ and $\mathrm{L}$ ). Resting blood glucose concentration increased in the IS group but not in the IR group (Figure $4 \mathrm{E}$ and $\mathrm{F}$ ).

The training intervention did not induce significant changes in plasma glycerol, insulin, lactate, adrenalin or noradrenalin concentrations. However, resting insulin concentrations, as well as insulin concentration during exercise and recovery remained elevated in the obese IR compared to obese IS group (Figure 4 I-J).

The exercise-induced increase in plasma FFA (Figure $4 \mathrm{C}$ and D), lactate (Figure $4 \mathrm{G}$ and $\mathrm{H}$ ) and adrenalin (Figure $4 \mathrm{M}$ and $\mathrm{N}$ ) concentrations were significantly blunted after intervention. Peak blood ANP concentrations (Figure $4 \mathrm{~K}$ and $\mathrm{L}$ ) tended to be reduced in both obese groups. The increase in plasma glycerol, insulin and glucose concentrations during exercise remained unchanged.

In the recovery period, beside reduced plasma FFA (Figure $4 \mathrm{C}$ and $\mathrm{D}$ ) and adrenalin concentrations (Figure $4 \mathrm{M}$ and $\mathrm{N}$ ), no significant training-induced changes were observed in plasma glycerol, glucose, lactate, ANP, noradrenalin or serum insulin concentrations (Figure 4). In addition, the training intervention did not induce changes in whole-body energy expenditure and substrate oxidation (Supplemental Figure 1 A-E). Detailed post-intervention systemic plasma responses at rest, during exercise and recovery are shown in Supplemental Table S4. 
A

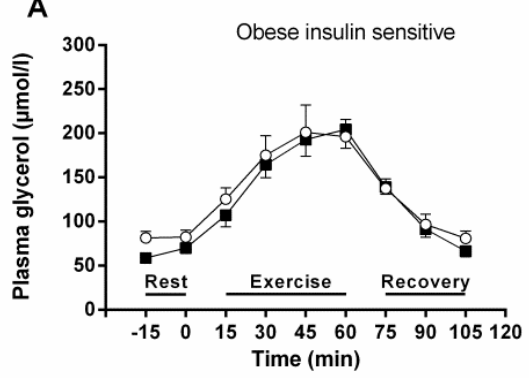

C

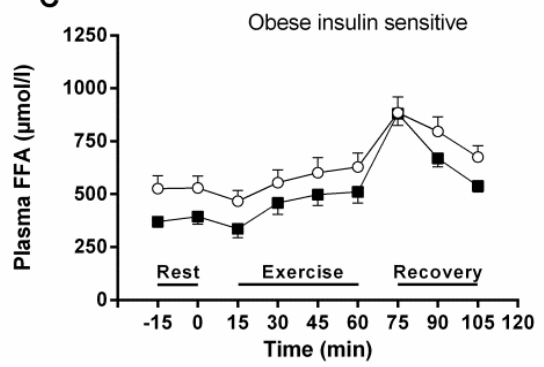

E

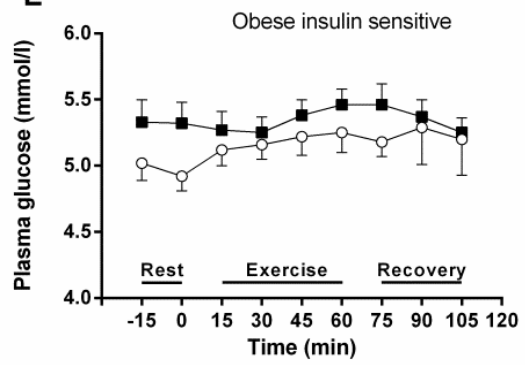

G

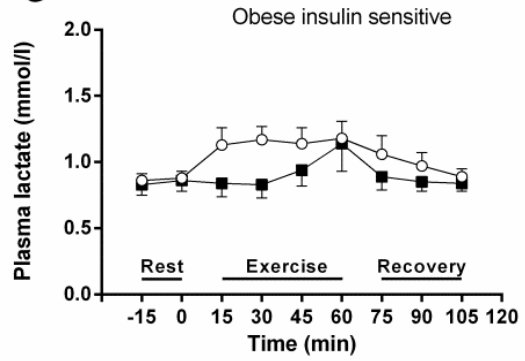

B
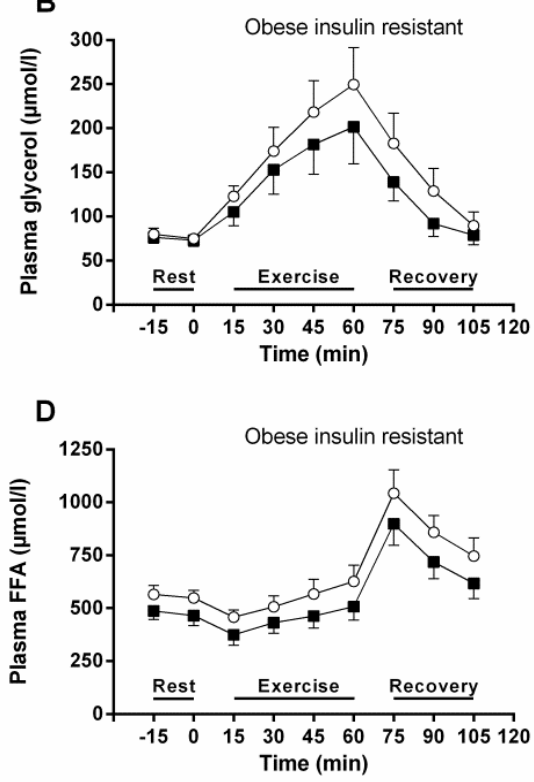

$\mathbf{F}$

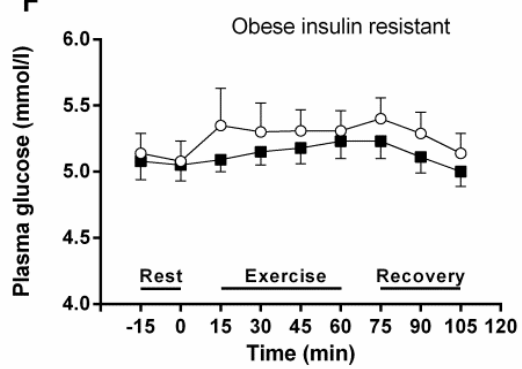

H

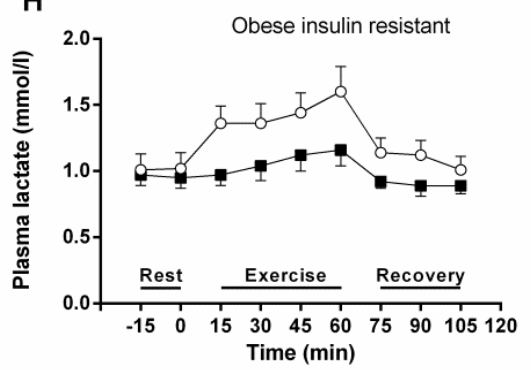

Figure 4 - Exercise training-induced changes in systemic plasma glycerol, FFA, glucose, lactate, ANP, adrenalin, noradrenalin and serum insulin in obese individuals. Systemic glycerol (A-B), FFA (C-D), glucose (E-F), lactate (G-H), insulin (I-J), ANP (K-L), adrenalin (M-N) and noradrenalin (O-P) of obese insulin sensitive and obese insulin resistant individuals at baseline (white circles) and after 12 weeks of exercise training intervention (black squares). Data are presented as mean \pm SEM. 
I

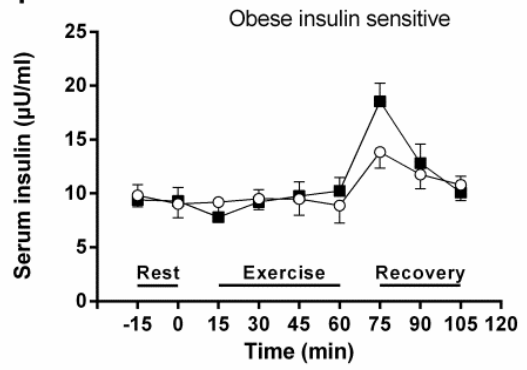

$\mathrm{K}$

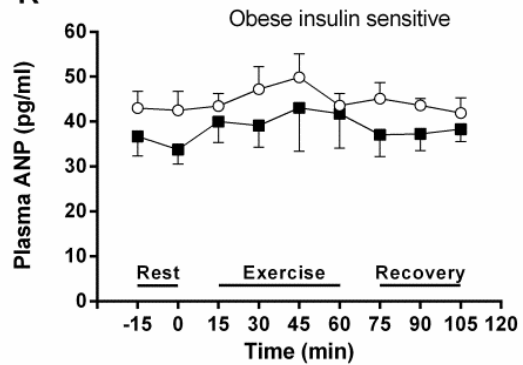

M

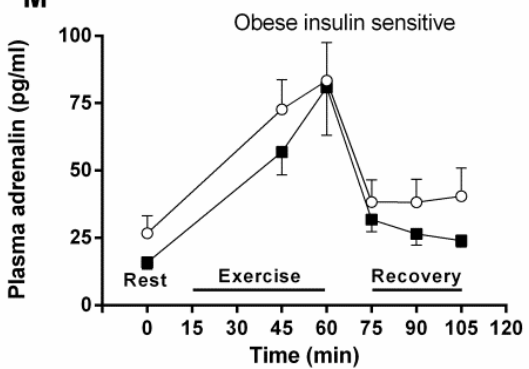

0

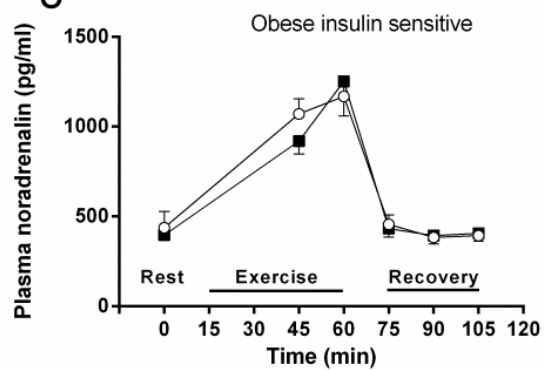

$\mathbf{J}$

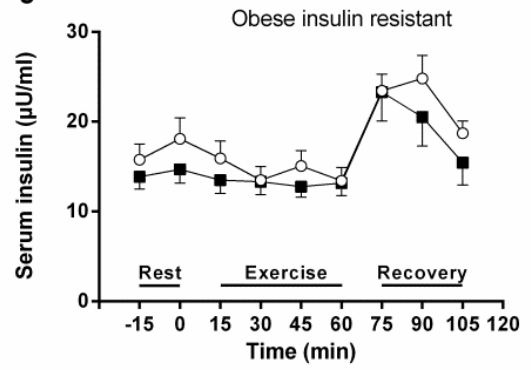

$\mathbf{L}$

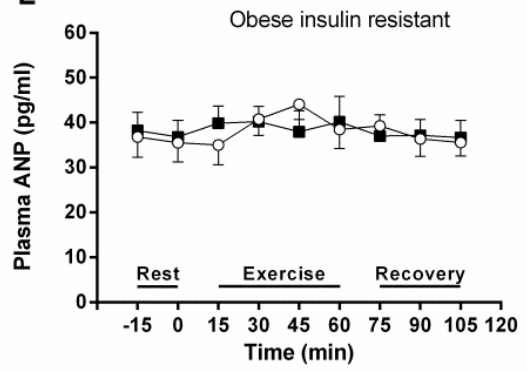

N
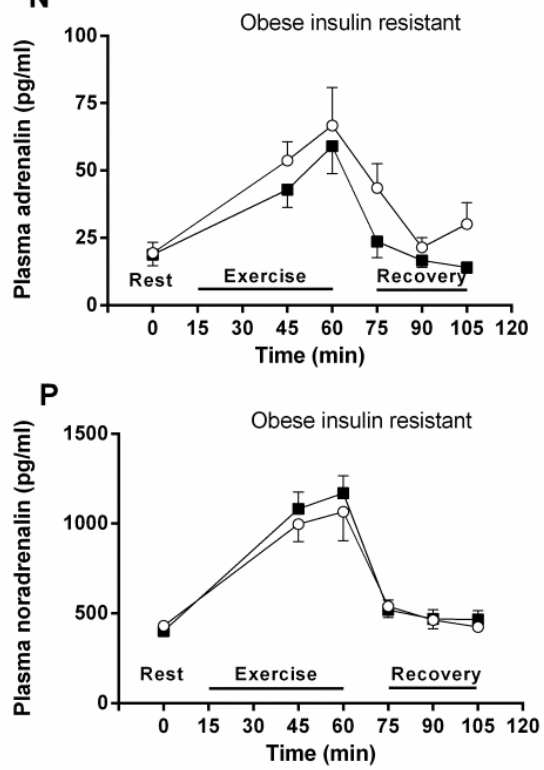

Figure 4 - continued. Exercise training-induced changes in systemic plasma glycerol, FFA, glucose, lactate, ANP, adrenalin, noradrenalin and serum insulin in obese individuals. Systemic glycerol (A-B), FFA (C-D), glucose (E-F), lactate (G-H), insulin (I-J), ANP (K-L), adrenalin (M-N) and noradrenalin (O-P) of obese insulin sensitive and obese insulin resistant individuals at baseline (white circles) and after 12 weeks of exercise training intervention (black squares). Data are presented as mean \pm SEM. 
Following exercise intervention, SCAT lipolysis was investigated in the obese IR group only. Ethanol out/in ratio (Figure $5 \mathrm{~A}$ ) as well as resting, exercise-induced and recovery-related extracellular glycerol concentration (Figure $5 \mathrm{~B}$ ) were not altered after exercise intervention. Additionally, $a-/ \beta$-adrenergic blockade had no significant effect on resting, exercise-induced or recovery-related extracellular glycerol concentration in SCAT following the exercise training program (Figure 5 B; Figure 3).

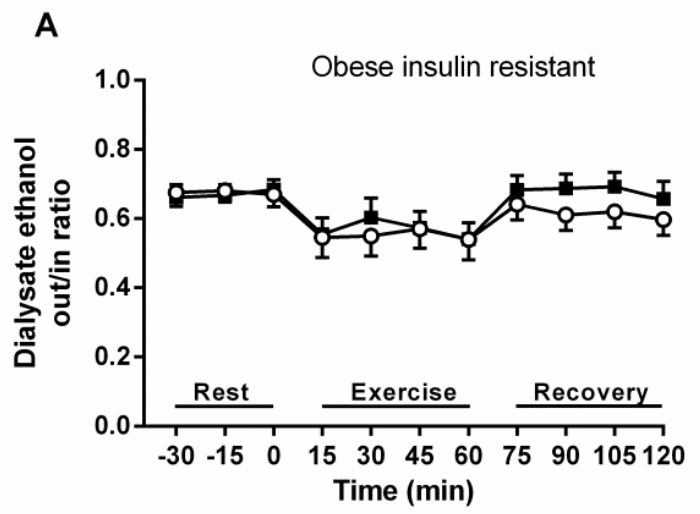

B

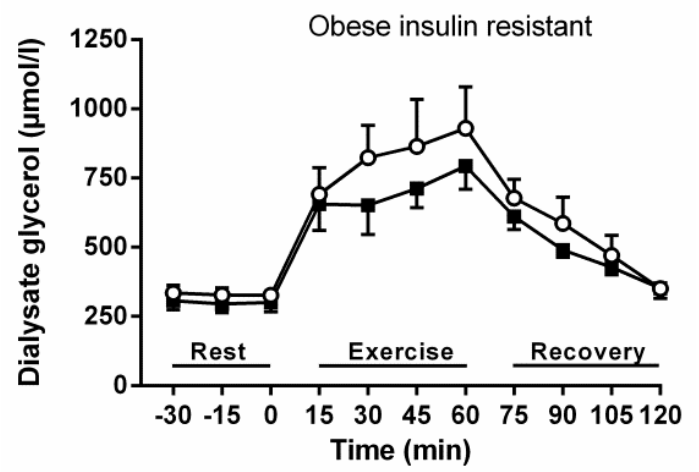

Figure 5 - Exercise training-induced changes in subcutaneous adipose tissue extracellular glycerol concentration and adipose tissue blood flow indices in obese insulin resistant individuals. Subcutaneous adipose tissue ethanol ratio (A) and extracellular glycerol concentration (B) in the obese insulin resistant individuals at rest, during exercise and recovery after 12 weeks of exercise training in control probe (white circles) and the probe perfused with phentolamine and propranolol (black squares). Data are presented as mean \pm SEM.

\section{Discussion}


The present study is the first to investigate (non-)adrenergically-mediated lipolysis during low-intensity endurance-type exercise in abdominal subcutaneous adipose tissue (SCAT) in middle-aged obese IS and obese IR men as compared to age-matched lean IS men. Furthermore, we examined whether a 12-week exercise intervention altered resting and exercise-induced (non)adrenergically-mediated SCAT lipolysis in obese IR individuals. Here, we demonstrated that the exercise-induced increase in abdominal SCAT extracellular glycerol concentration (reflecting local lipolysis) was more pronounced in obese as compared to lean individuals, which may at least partly be explained by the higher adipose tissue blood flow (ATBF) in lean individuals. Exercise-induced SCAT lipolysis was substantially reduced ( 40\%) following local combined $a-/ \beta$-adrenergic blockade in obese IS individuals, but not in obese IR or lean IS individuals. Finally, the exercise training intervention improved body composition, physical fitness and exercise-induced systemic responses in both obese groups, and insulin sensitivity in the obese IR group. However, this was not accompanied by changes in adrenergically- and nonadrenergically-mediated lipolysis in the SCAT of obese IR individuals. Collectively, our findings indicate that exercise-induced lipolysis is predominantly mediated by non-adrenergic factors, most likely mediated by natriuretic peptides (NP) in middle-aged lean IS, obese IS and obese IR individuals.

The present study showed a more pronounced increase in exercise-mediated SCAT extracellular glycerol concentration in obese IS as compared to lean individuals. Although the lipolytic response in abdominal SCAT is often blunted in human obesity ${ }^{9}$, the higher exercise-induced extracellular glycerol levels in both obese groups is likely explained by the substantially lower ATBF in the obese state, which contributes to higher extracellular glycerol concentrations due to a lower removal of glycerol from the AT ${ }^{16,44}$. Local a-/ $\beta$-adrenergic blockade substantially reduced basal ATBF in lean but not in the obese individuals. This might suggest that adrenergic sensitivity of ATBF is reduced in the obese state, which is in line with previous findings 39,45 . Thus, these differences in ATBF between lean and obese individuals should be taken into account when interpreting local SCAT lipolysis.

Interestingly, combined $a-/ \beta$-adrenergic receptor blockade reduced exercise- 
induced SCAT lipolysis in obese IS men but not in lean and obese IR individuals. In obese IR individuals, the lipolytic activity of the $\beta$-adrenergic receptors is attenuated $9,11,12$, while an increase in anti-lipolytic $a_{2}$-adrenergic receptors in SCAT reduce exercise-mediated lipolysis ${ }^{39}$. In addition, obese IR individuals often display lower plasma catecholamine (adrenaline, noradrenaline) responses to physical exercise ${ }^{46}$, although the latter was not observed in the current study. Therefore, the reduced exercise-mediated lipolytic response upon combined $a-/ \beta$-adrenergic receptor blockade in the obese IS individuals, as opposed to the obese IR group, might suggest differences in adrenergic receptor expression and sensitivity. The blunted lipolytic response in obese IR individuals might also be explained by the significantly higher fasting and exercise induced serum insulin levels. Since the anti-lipolytic effects of insulin might be normal or only slightly impaired in obese AT ${ }^{47-49}$, the observed hyperinsulinemia in the obese IR group might have contributed to the attenuated adrenergicallymediated SCAT lipolysis as compared to the obese IS group, as previously shown ${ }^{50}$. In addition, increased plasma lactate concentrations in obese IR individuals, may have contributed to the reduced lipolytic response, since lactate has been shown to inhibit lipolysis in mice ${ }^{51}$ and human primary adipocytes in vitro ${ }^{18}$.

The present study implies that non-adrenergic regulators of lipolysis play a major role during low-intensity endurance-type exercise. It has previously been demonstrated that propranolol 28 and phentolamine 39,40 fully inhibit adrenergically-mediated SCAT lipolysis at the concentrations used in the present study. The exercise-induced lipolytic response was only suppressed to a minor extent in all groups, clearly indicating a major contribution of non-adrenergic components to SCAT lipolysis during exercise. Importantly, other (anti-)lipolytic factors, such as the parathyroid hormone, cortisol and growth hormone are less important during the type and duration of exercise as applied in the current study ${ }^{7,16}$. Noteworthy, atrial natriuretic peptide (ANP) may be responsible for the exercise-induced increase in SCAT lipolysis in the present study, especially since ANP is one of the major lipolytic hormones produced upon exercise ${ }^{26,52}$, next to sympathetic nervous system activation. In line with our findings, it has previously been shown that non-adrenergically mechanisms are involved in SCAT lipolysis, accounting for $\sim 65 \%$ of the exercise-mediated lipolysis in young 
healthy lean and overweight men 15,16. Previously, a lower in vivo ANP responsiveness in SCAT of overweight ${ }^{31}$ and obese ${ }^{30}$ individuals was observed. In line, the non-adrenergically regulation of SCAT lipolysis has been reported to be more pronounced in young healthy overweight as compared to lean men ${ }^{16}$, suggesting that ANP-mediated SCAT lipolysis is particularly important in the overweight and obese state. Interestingly, in contrast to obese IS individuals, we found that SCAT lipolysis in obese IR men was not affected by local $a-/ \beta$ adrenergic blockade, which may propose an interaction between SCAT adrenergically-mediated lipolysis and whole-body insulin resistance. These findings support a catecholamine-resistance phenotype of the SCAT during exercise especially in the obese IR state. Therefore, a major role for ANP in SCAT lipolysis during exercise can be suggested, which is sustained in the obese IR state.

To ensure adequate fatty acid delivery to the working skeletal muscles during exercise, the sustained ANP-mediated lipolytic response, the adrenergicallymediated lipolysis and a blunted insulin- and lactate-mediated inhibition of lipolysis, might have contributed to the more pronounced SCAT lipolysis as observed in the obese IS men. Because most of the anti-lipolytic action of insulin is mediated through stimulation and activation of cellular phosphodiesterase-3B 53,54, which degrades CAMP, attenuation of the ANP-related SCAT lipolysis by insulin is unlikely, since the latter is cGMP-mediated 27 . Likewise, it has been shown that NP receptor expression (both functional NPRA and scavenging NPRC) in SCAT associates with whole-body insulin sensitivity ${ }^{55}$.

The 12-week exercise intervention increased insulin sensitivity in the obese IR group. While plasma glycerol, FFA, lactate, ANP and adrenalin concentrations were significantly reduced after the intervention, only minor reductions were observed for circulating glucose, insulin and noradrenalin concentrations. However, local exercise-induced abdominal SCAT lipolysis was not improved following the exercise intervention. Moreover, the efficiency of local $a-/ \beta$ adrenergic blockade was not affected by the exercise intervention. Together, these data suggest that even after a substantial improvement in metabolic profile (as indicated by our systemic responses) and body composition after a 12-week exercise intervention, lipolytic disturbances remain unaffected in SCAT 
of obese IR individuals. Optimized therapies are warranted to achieve enhancements in the regulation of SCAT lipolysis, especially in metabolically compromised individuals.

Although evidence suggests that exercise training induces beneficial changes in SCAT insulin sensitivity ${ }^{56}$, SCAT adrenergic sensitivity ${ }^{56-58}$ as well as ANPmediated lipolysis ${ }^{59}$, future mechanistic studies are needed to obtain a better understanding of the hormonal (exercise-induced) lipolytic regulation in lean and obese individuals with a different degree of insulin sensitivity. Unfortunately, a NPRA receptor agonist/antagonist for use in humans is currently unavailable, which hampers strong conclusions about the physiological role of ANP in human AT lipolysis in vivo.

\section{Conclusion}


In conclusion, this study is the first to report the integrated physiological role of adrenergically- and non-adrenergically-mediated SCAT lipolysis during lowintensity endurance-type exercise in middle-aged lean IS, obese IS and obese IR men, and the effects of a 12-week supervised exercise intervention on these processes in obese individuals. The present data demonstrate a major role for non-adrenergically regulated lipolysis in SCAT during low-intensity exercise, likely involving ANP-mediated lipolysis. Furthermore, the exercise training intervention was not accompanied by changes in SCAT lipolysis in obese IR individuals, regardless of improvements in metabolic profile and body composition.

\section{Acknowledgments}

The authors would like to thank all participants in this study and J. Stegen, W. Sluijsmans, H. Aydeniz, and Y. Essers for their excellent analytical and technical support (all from the Department of Human Biology, NUTRIM School of Nutrition and Translational Research in Metabolism, Maastricht University Medical Center ${ }^{+}$, the Netherlands) and B. Froyen for his assistance in the experimental testing and training supervision as well as the department of ReGO (Jessa Hospital) for their accommodation.

\section{Funding}

Part of this work was financed by the Research Foundation - Flanders (FWO) (KAN 1507217N).

\section{References}


1. Goossens G (2008) The role of adipose tissue dysfunction in the pathogenesis of obesity-related insulin resistance. Physiol Behav 94:206-218

2. Lafontan M (2014) Adipose tissue and adipocyte dysregulation. Diabetes Metab 40:1628

3. Stinkens R, Goossens G, Jocken J, Blaak E (2015) Targeting fatty acid metabolism to improve glucose metabolism. Obes Rev 16:715-757

4. Frayn K (2002) Adipose tissue as a buffer for daily lipid flux. Diabetologia 45:12011210

5. Jocken J, Blaak E (2008) Catecholamine-induced lipolysis in adipose tissue and skeletal muscle in obesity. Physiol Behav 94:219-230

6. Fruhbeck G, Mendez-Gimenez L, Fernandez-Formoso J, Fernandez S, Rodriguez A (2014) Regulation of adipocyte lipolysis. Nutr Res Rev 27:63-93

7. Hansen D, Meeusen R, Mullens A, Dendale P (2012) Effect of acute endurance and resistance exercise on endocrine hormones directly related to lipolysis and skeletal muscle protein synthesis in adult individuals with obesity. Sports Med 42:415-431

8. Ryden M, Jocken J, van Harmelen V, Dicker A, Hoffstedt J, Wiren M et al (2007) Comparative studies of the role of hormone-sensitive lipase and adipose triglyceride lipase in human fat cell lipolysis. Am J Physiol Endocrinol Metab 292:E1847-E1855

9. Jocken J, Goossens G, van Hees A, Frayn K, van Baak M, Stegen J et al (2008) Effect of beta-adrenergic stimulation on whole-body and abdominal subcutaneous adipose tissue lipolysis in lean and obese men. Diabetologia 51:320-327

10. Mauriege P, Despres J, Prud'homme D, Pouliot M, Marcotte M, Tremblay A et al (1991) Regional variation in adipose tissue lipolysis in lean and obese men. J Lipid Res 32:1625-1633

11. Reynisdottir S, Wahrenberg H, Carlstrom K, Rossner S, Arner P (1994) Catecholamine resistance in fat cells of women with upper-body obesity due to decreased expression of beta 2-adrenoceptors. Diabetologia 37:428-435

12. Lafontan M, Berlan M (1993) Fat cell adrenergic receptors and the control of white and brown fat cell function. J Lipid Res 34:1057-1091

13. Arner $P$, Kriegholm E, Engfeldt $P$, Bolinder J (1990) Adrenergic regulation of lipolysis in situ at rest and during exercise. J Clin Invest 85:893-898

14. Hellstrom L, Blaak E, Hagstrom-Toft E (1996) Gender differences in adrenergic regulation of lipid mobilization during exercise. Int J Sports Med 17:439-447

15. Moro C, Polak J, Hejnova J, Klimcakova E, Crampes F, Stich V et al (2006) Atrial natriuretic peptide stimulates lipid mobilization during repeated bouts of endurance exercise. Am J Physiol Endocrinol Metab 290:E864-E869

16. Moro C, Pillard F, de Glisezinski I, Klimcakova E, Crampes F, Thalamas C et al (2008) Exercise-induced lipid mobilization in subcutaneous adipose tissue is mainly related to natriuretic peptides in overweight men. Am J Physiol Endocrinol Metab 295:E505-E513

17. Lafontan M (2005) Fat cells: afferent and efferent messages define new approaches to treat obesity. Annu Rev Pharmacol Toxicol 45:119-146

18. Liu C, Wu J, Zhu J, Kuei C, Yu J, Shelton J et al (2009) Lactate inhibits lipolysis in fat cells through activation of an orphan G-protein-coupled receptor, GPR81. J Biol Chem 284:2811-2822

19. Langin D (2010) Adipose tissue lipolysis revisited (again!): lactate involvement in insulin antilipolytic action. Cell Metab 11:242-243

20. Moro $C$ (2016) Targeting cardiac natriuretic peptides in the therapy of diabetes and obesity. Expert Opin Ther Targets 20:1445-1452

21. Wang T, Larson M, Levy D, Benjamin E, Leip E, Wilson P et al (2004) Impact of obesity on plasma natriuretic peptide levels. Circulation 109:594-600 
22. Das S, Drazner M, Dries D, Vega G, Stanek H, Abdullah S et al (2005) Impact of body mass and body composition on circulating levels of natriuretic peptides: results from the Dallas Heart Study. Circulation 112:2163-2168

23. Khan A, Cheng S, Magnusson M, Larson M, Newton-Cheh C, McCabe E et al (2011) Cardiac natriuretic peptides, obesity, and insulin resistance: evidence from two community-based studies. J Clin Endocrinol Metab 96:3242-3249

24. Magnusson M, Jujic A, Hedblad B, Engstrom G, Persson M, Struck J et al (2012) Low plasma level of atrial natriuretic peptide predicts development of diabetes: the prospective Malmo Diet and Cancer study. J Clin Endocrinol Metab 97:638-645

25. Gruden G, Landi A, Bruno G (2014) Natriuretic peptides, heart, and adipose tissue: new findings and future developments for diabetes research. Diabetes Care 37:28992908

26. Sengenes C, Berlan M, De Glisezinski I, Lafontan M, Galitzky J (2000) Natriuretic peptides: a new lipolytic pathway in human adipocytes. FASEB J 14:1345-1351

27. Sengenes C, Bouloumie A, Hauner H, Berlan M, Busse R, Lafontan M et al (2003) Involvement of a cGMP-dependent pathway in the natriuretic peptide-mediated hormone-sensitive lipase phosphorylation in human adipocytes. J Biol Chem 278:48617-48626

28. Moro C, Crampes F, Sengenes C, De Glisezinski I, Galitzky J, Thalamas C et al (2004) Atrial natriuretic peptide contributes to physiological control of lipid mobilization in humans. FASEB J 18:908-910

29. Verboven K, Hansen D, Moro C, Eijnde BO, Hoebers N, Knol J et al (2016). Attenuated atrial natriuretic peptide-mediated lipolysis in subcutaneous adipocytes of obese type 2 diabetic men. Clin Sci (Lond) 130:1105-1114

30. Ryden M, Backdahl J, Petrus P, Thorell A, Gao H, Coue M et al (2016) Impaired atrial natriuretic peptide-mediated lipolysis in obesity. Int J Obes (Lond) 40:714-720

31. Stich V, de Glisezinski I, Crampes F, Suljkovicova H, Galitzky J, Riviere D et al (1999) Activation of antilipolytic alpha(2)-adrenergic receptors by epinephrine during exercise in human adipose tissue. Am J Physiol 277:R1076-R1083

32. Stich V, de Glisezinski I, Galitzky J, Hejnova J, Crampes F, Riviere D et al (1999) Endurance training increases the beta-adrenergic lipolytic response in subcutaneous adipose tissue in obese subjects. Int J Obes Relat Metab Disord 23:374-381

33. Polak J, Klimcakova E, Moro C, Viguerie N, Berlan M, Hejnova J et al (2006) Effect of aerobic training on plasma levels and subcutaneous abdominal adipose tissue gene expression of adiponectin, leptin, interleukin 6, and tumor necrosis factor alpha in obese women. Metabolism 55:1375-1381

34. Moro C, Pillard F, De Glisezinski I, Harant I, Riviere D, Stich V et al (2005) Training Enhances ANP Lipid-Mobilizing Action in Adipose Tissue of Overweight Men. Medicine \& Science in Sports \& Exercise 37:1126-1132

35. Matthews D, Hosker J, Rudenski A, Naylor B, Treacher D, Turner R (1985) Homeostasis model assessment: insulin resistance and beta-cell function from fasting plasma glucose and insulin concentrations in man. Diabetologia 28:412-419

36. Blaak E, Hul G, Verdich C, Stich V, Martinez A, Petersen M et al (2006) Fat oxidation before and after a high fat load in the obese insulin-resistant state. J Clin Endocrinol Metab 91:1462-1469

37. Wildman R, Muntner P, Reynolds K, McGinn A, Rajpathak S, Wylie-Rosett J et al (2008) The obese without cardiometabolic risk factor clustering and the normal weight with cardiometabolic risk factor clustering: prevalence and correlates of 2 phenotypes among the US population (NHANES 1999-2004). Arch Intern Med 168:1617-1624 
38. Hickner R, Rosdahl H, Borg I, Ungerstedt U, Jorfeldt L, Henriksson J (1991) Ethanol may be used with the microdialysis technique to monitor blood flow changes in skeletal muscle: dialysate glucose concentration is blood-flow-dependent. Acta Physiol Scand 143:355-356

39. Stich V, De Glisezinski I, Crampes F, Hejnova J, Cottet-Emard J, Galitzky J et al (2000) Activation of alpha(2)-adrenergic receptors impairs exercise-induced lipolysis in SCAT of obese subjects. Am J Physiol Regul Integr Comp Physiol 279:R499-R504.

40. Polak J, Moro C, Bessiere D, Hejnova J, Marques MA, Bajzova M et al (2007) Acute exposure to long-chain fatty acids impairs \{alpha\}2-adrenergic receptor-mediated antilipolysis in human adipose tissue. J Lipid Res 48:2236-2246

41. Frayn K (1983) Calculation of substrate oxidation rates in vivo from gaseous exchange. J Appl Physiol Respir Environ Exerc Physiol 55:628-634

42. Weir J (1949) New methods for calculating metabolic rate with special reference to protein metabolism. J Physiol 109:1-9

43. Yumuk V, Tsigos C, Fried M, Schindler K, Busetto L, Micic D et al (2015) European Guidelines for Obesity Management in Adults. Obes Facts 8:402-424

44. Bulow J (1981) Human adipose tissue blood flow during prolonged exercise, III. Effect of beta-adrenergic blockade, nicotinic acid and glucose infusion. Scand J Clin Lab Invest 41:415-424

45. Ardilouze J, Karpe F, Currie J, Frayn K, Fielding B (2004) Subcutaneous adipose tissue blood flow varies between superior and inferior levels of the anterior abdominal wall. Int J Obes Relat Metab Disord 28:228-233

46. Zouhal H, Lemoine-Morel S, Mathieu M, Casazza G, Jabbour G (2013) Catecholamines and obesity: effects of exercise and training. Sports Med 43:591-600

47. Lafontan M, Langin D (2009) Lipolysis and lipid mobilization in human adipose tissue. Prog Lipid Res 48:275-297

48. Jocken J, Goossens G, Boon H, Mason R, Essers Y, Havekes B et al (2013) Insulinmediated suppression of lipolysis in adipose tissue and skeletal muscle of obese type 2 diabetic men and men with normal glucose tolerance. Diabetologia 56:2255-2265

49. Hershkop K, Besor O, Santoro N, Pierpont B, Caprio S, Weiss R (2016) Adipose Insulin Resistance in Obese Adolescents Across the Spectrum of Glucose Tolerance. J Clin Endocrinol Metab 101:2423-2431

50. Zhang J, Hupfeld CJ, Taylor SS, Olefsky JM, Tsien RY. Insulin disrupts beta-adrenergic signalling to protein kinase $A$ in adipocytes. Nature 437:569-573

51. Ahmed K, Tunaru S, Tang C, Muller M, Gille A, Sassmann A et al (2010). An autocrine lactate loop mediates insulin-dependent inhibition of lipolysis through GPR81. Cell Metab 11:311-319

52. Follenius M, Brandenberger G (1988) Increase in atrial natriuretic peptide in response to physical exercise. Eur J Appl Physiol Occup Physiol 57:159-62

53. Lonnroth $P$, Smith $U$ (1986) The antilipolytic effect of insulin in human adipocytes requires activation of the phosphodiesterase. Biochem Biophys Res Commun 141:1157-1161

54. Makino H, Suzuki T, Kajinuma H, Yamazaki M, Ito H, Yoshida S (1992) The role of insulin-sensitive phosphodiesterase in insulin action. Adv Second Messenger Phosphoprotein Res 25:185-199

55. Kovacova Z, Tharp WG, Liu D, Wei W, Xie H, Collins S et al (2016) Adipose tissue natriuretic peptide receptor expression is related to insulin sensitivity in obesity and diabetes. Obesity (Silver Spring) 24:820-828

56. Polak J, Moro C, Klimcakova E, Hejnova J, Majercik M, Viguerie N et al (2005) Dynamic strength training improves insulin sensitivity and functional balance between 
adrenergic alpha 2A and beta pathways in subcutaneous adipose tissue of obese subjects. Diabetologia 48:2631-2640

57. De Glisezinski I, Crampes F, Harant I, Berlan M, Hejnova J, Langin D et al (1998) Endurance training changes in lipolytic responsiveness of obese adipose tissue. Am J Physiol 275:E951-E956

58. Richterova B, Stich V, Moro C, Polak J, Klimcakova E, Majercik M et al (2004) Effect of endurance training on adrenergic control of lipolysis in adipose tissue of obese women. J Clin Endocrinol Metab 89:1325-1331

59. Moro C, Pasarica M, Elkind-Hirsch K, Redman LM (2009) Aerobic exercise training improves atrial natriuretic peptide and catecholamine-mediated lipolysis in obese women with polycystic ovary syndrome. J Clin Endocrinol Metab 94:2579-2586 
A

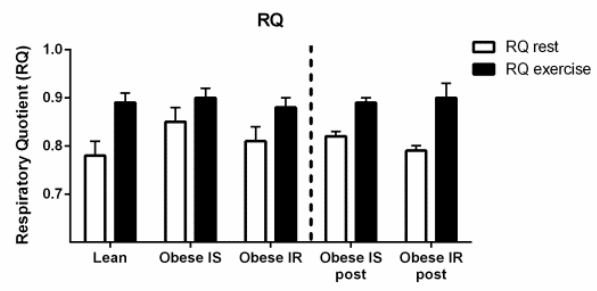

B

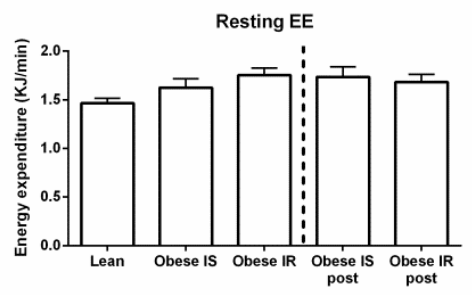

D

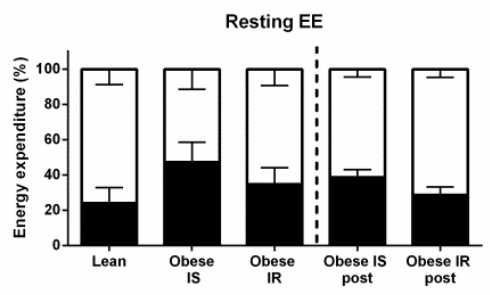

C

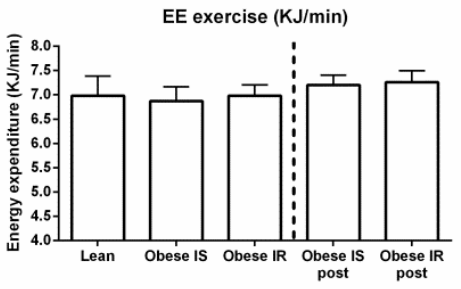

E

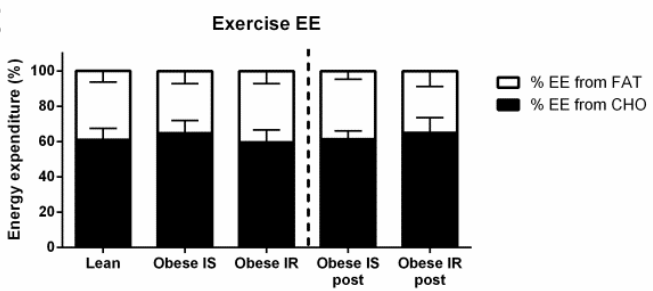

Supplemental Figure 1 - Fasting and exercise-induced whole-body energy expenditure and substrate oxidation before and after exercise intervention. Indirect calorimetry was performed during resting conditions and during an acute exercise bout of moderate intensity. Mean $\mathrm{O}_{2}-$ consumption and $\mathrm{CO}_{2}$-production over $10 \mathrm{~min}$ were used for calculations of respiratory quotient (A), energy expenditure and substrate oxidation during fasting $(B+D)$ and during exercise $(C+E)$. Post intervention data are added for both obese groups (A-E). CHO, carbohydrates; $E E$, energy expenditure; IR, insulin resistant; IS, insulin sensitive. Data are presented as mean \pm SEM. 


\begin{tabular}{|c|c|c|c|c|c|c|c|}
\hline \multirow[t]{2}{*}{ Supplemental Table S1. } & \multicolumn{7}{|c|}{$\begin{array}{l}\text { Average concentrations of circulating glycerol, insulin, FFA, glucose, lactate, ANP, adrenalin and noradrenalin at rest, } \\
\text { during } 1 \mathrm{~h} \text { of exercise and } 1 \mathrm{~h} \text { of recovery in obese insulin sensitive, obese insulin resistant and lean individuals }\end{array}$} \\
\hline & $\begin{array}{l}\text { Lean } \\
(n=10)\end{array}$ & $\begin{array}{l}\text { Obese insulin sensitive } \\
(n=10)\end{array}$ & $\begin{array}{c}\mathrm{P} \\
\text { (vs lean) }\end{array}$ & $\begin{array}{l}\text { Obese insulin resistant } \\
(n=10)\end{array}$ & $\begin{array}{c}\mathrm{P} \\
\text { (vs lean) }\end{array}$ & $\begin{array}{c}\mathrm{P} \\
\text { (vs OB IS) }\end{array}$ & $\begin{array}{l}\text { ANOVA } \\
\text { P-value }\end{array}$ \\
\hline \multicolumn{8}{|l|}{ GLYCEROL } \\
\hline Rest $(\mu \mathrm{mol} / \mathrm{l})$ & $70 \pm 4$ & $81 \pm 7$ & 0.580 & $77 \pm 5$ & 1.000 & 1.000 & 0.416 \\
\hline Exercise $\left(\mathrm{AUC}_{0-60}\right)(\mu \mathrm{mol} / \mathrm{I} * 60 \mathrm{~min})$ & $136 \pm 12$ & $163 \pm 19$ & 0.978 & $168 \pm 22$ & 0.722 & 1.000 & 0.448 \\
\hline \multicolumn{8}{|l|}{ INSULIN } \\
\hline Rest $(\mu \mathrm{U} / \mathrm{ml})$ & $7.0 \pm 0.6$ & $9.4 \pm 1.1$ & 0.563 & $16.9 \pm 1.8$ & $<0.001$ & 0.001 & $<0.001$ \\
\hline Exercise $\left(\mathrm{AUC}_{0-60}\right)(\mu \mathrm{U} / \mathrm{ml} * 60 \mathrm{~min})$ & $7.4 \pm 0.64$ & $9.3 \pm 1.2$ & 0.791 & $15.0 \pm 1.5$ & $<0.001$ & 0.005 & $<0.001$ \\
\hline Recovery $\left(\mathrm{AUC}_{75-105}\right)(\mu \mathrm{U} / \mathrm{ml} * 45 \mathrm{~min})$ & $8.5 \pm 1.0$ & $11.7 \pm 1.2$ & 0.209 & $21.4 \pm 1.3$ & $<0.001$ & $<0.001$ & $<0.001$ \\
\hline \multicolumn{8}{|l|}{ FFA } \\
\hline Rest $(\mu \mathrm{mol} / \mathrm{l})$ & $451 \pm 31$ & $528 \pm 58$ & 0.658 & $557 \pm 36$ & 0.292 & 1.000 & 0.224 \\
\hline Exercise $\left(\mathrm{AUC}_{0-60}\right)(\mu \mathrm{mol} / \mathrm{l} * 60 \mathrm{~min})$ & $466 \pm 42$ & $555 \pm 56$ & 0.628 & $531 \pm 47$ & 1.000 & 1.000 & 0.425 \\
\hline Recovery $\left(A \cup C_{75-105}\right)(\mu \mathrm{mol} / \mathrm{I} * 45 \mathrm{~min})$ & $601 \pm 37$ & $777 \pm 64$ & 0.189 & $864 \pm 82$ & 0.019 & 1.000 & 0.020 \\
\hline \multicolumn{8}{|l|}{ GLUCOSE } \\
\hline Rest $(\mathrm{mmol} / \mathrm{l})$ & $5.2 \pm 0.1$ & $5.0 \pm 0.1$ & 0.722 & $5.1 \pm 0.1$ & 1.000 & 1.000 & 0.488 \\
\hline Exercise $\left(A \cup C_{0-60}\right)(\mathrm{mmol} / / * 60 \mathrm{~min})$ & $5.3 \pm 0.1$ & $5.1 \pm 0.1$ & 1.000 & $5.3 \pm 0.2$ & 1.000 & 1.000 & 0.679 \\
\hline \multicolumn{8}{|l|}{ LACTATE } \\
\hline Rest $(\mathrm{mmol} / \mathrm{l})$ & $0.9 \pm 0.1$ & $0.9 \pm 0.05$ & 1.000 & $1.0 \pm 0.1$ & 0.628 & 0.600 & 0.339 \\
\hline Exercise $\left(\mathrm{AUC}_{0-60}\right)(\mathrm{mmol} / \mathrm{I} * 60 \mathrm{~min})$ & $1.0 \pm 0.7$ & $1.1 \pm 0.1$ & 0.100 & $1.4 \pm 0.1$ & 0.044 & 0.313 & 0.044 \\
\hline \multicolumn{7}{|l|}{ ANP } & 0.061 \\
\hline Rest $(\mathrm{pg} / \mathrm{ml})$ & $43.8 \pm 5.2$ & $41.7 \pm 3.2$ & 1.000 & $36.2 \pm 4.0$ & 0.548 & 0.793 & 0.356 \\
\hline Exercise $\left(\mathrm{AUC}_{0-60}\right)(\mathrm{pg} / \mathrm{ml} * 60 \mathrm{~min})$ & $47.2 \pm 4.8$ & $45.9 \pm 3.5$ & 1.000 & $39.2 \pm 2.2$ & 0.452 & 0.680 & 0.300 \\
\hline Recovery $\left(A \cup C_{75-105}\right)(\mathrm{pg} / \mathrm{ml} * 45 \mathrm{~min})$ & $42.7 \pm 4.4$ & $44.0 \pm 2.3$ & 1.000 & $37.5 \pm 3.2$ & 0.864 & 0.616 & 0.390 \\
\hline \multicolumn{8}{|l|}{ ADRENALIN } \\
\hline Rest $(\mathrm{pg} / \mathrm{ml})$ & $16.4 \pm 3.8$ & $26.7 \pm 6.5$ & 0.561 & $19.4 \pm 4.0$ & 1.000 & 1.000 & 0.376 \\
\hline Exercise $\left(\mathrm{AUC}_{0-60}\right)(\mathrm{pg} / \mathrm{ml} * 60 \mathrm{~min})$ & $54.0 \pm 5.7$ & $76.2 \pm 11.2$ & 0.233 & $58.0 \pm 8.1$ & 1.000 & 0.429 & 0.171 \\
\hline Recovery $\left(\mathrm{AUC}_{75-105}\right)(\mathrm{pg} / \mathrm{ml} * 45 \mathrm{~min})$ & $35.2 \pm 3.2$ & $48.2 \pm 9.0$ & 0.537 & $39.4 \pm 5.9$ & 1.000 & 1.000 & 0.380 \\
\hline \multicolumn{8}{|l|}{ NORADRENALIN } \\
\hline Rest $(\mathrm{pg} / \mathrm{ml})$ & $493 \pm 85$ & $437 \pm 91$ & 1.000 & $430 \pm 42$ & 1.000 & 1.000 & 0.816 \\
\hline Exercise $\left(\mathrm{AUC}_{0-60}\right)(\mathrm{pg} / \mathrm{m} / * 60 \mathrm{~min})$ & $1107 \pm 81$ & $1095 \pm 83$ & 1.000 & $1007 \pm 110$ & 1.000 & 1.000 & 0.708 \\
\hline Recovery $\left(\right.$ AUC $\left._{75-105}\right)(\mathrm{pg} / \mathrm{ml} * 45 \mathrm{~min})$ & $610 \pm 71$ & $540 \pm 48$ & 1.000 & $580 \pm 56$ & 1.000 & 1.000 & 0.725 \\
\hline
\end{tabular}

Data are mean $\pm \mathrm{SEM} . \mathrm{AUC}_{0-60}$ (area under the curve during exercise (from timepoint 0 till $60 \mathrm{~min}$ )), $\mathrm{AUC}_{75-105}$ (area under the curve during the recovery period (from timepoint 75 until $105 \mathrm{~min})$ ). Group effects were tested with a one-way ANOVA with Bonferroni post-hoc. 


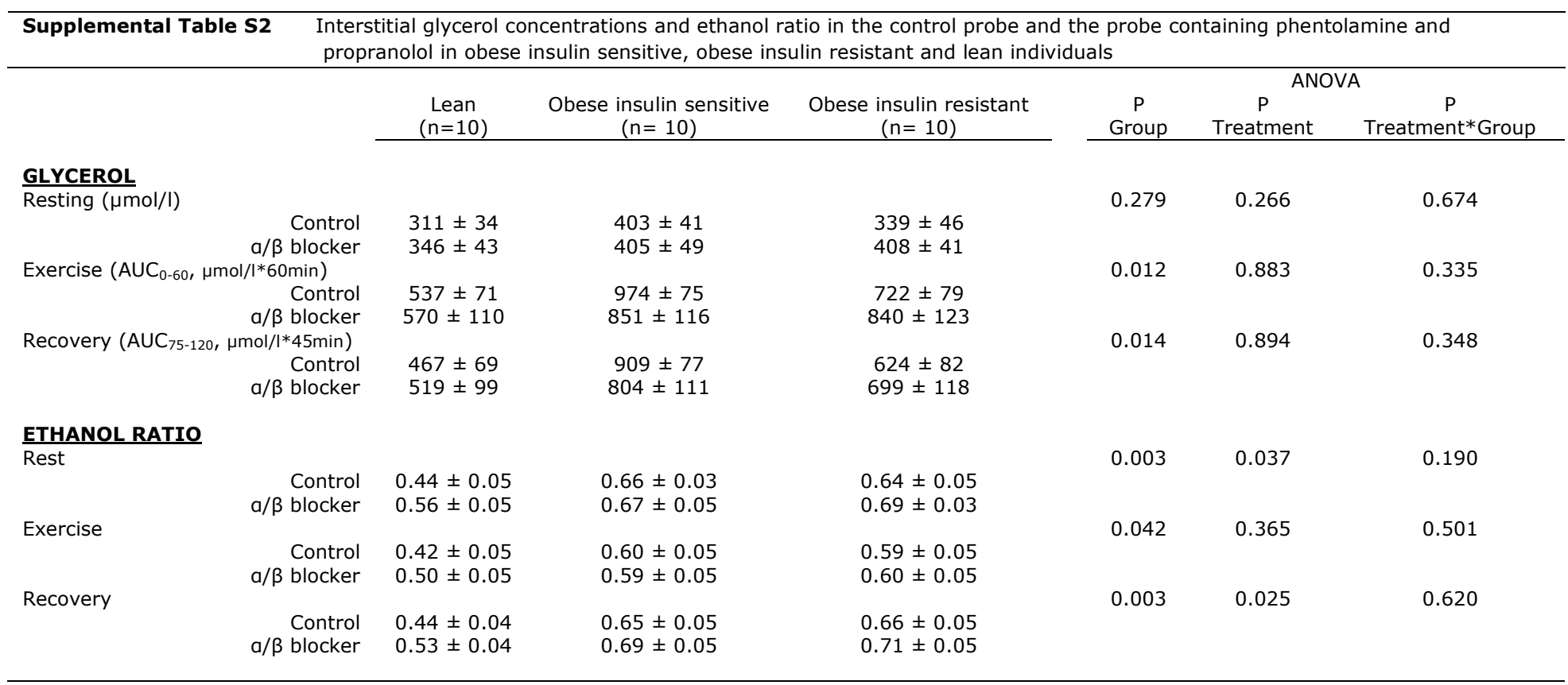

Data are expressed as mean \pm SEM. Control (probe containing Ringer), a-/ $\beta$-adrenergic blocker (probe containing Phentolamine + Propranolol).

$\mathrm{P}$ values were calculated via a 2-way repeated ANOVA. 


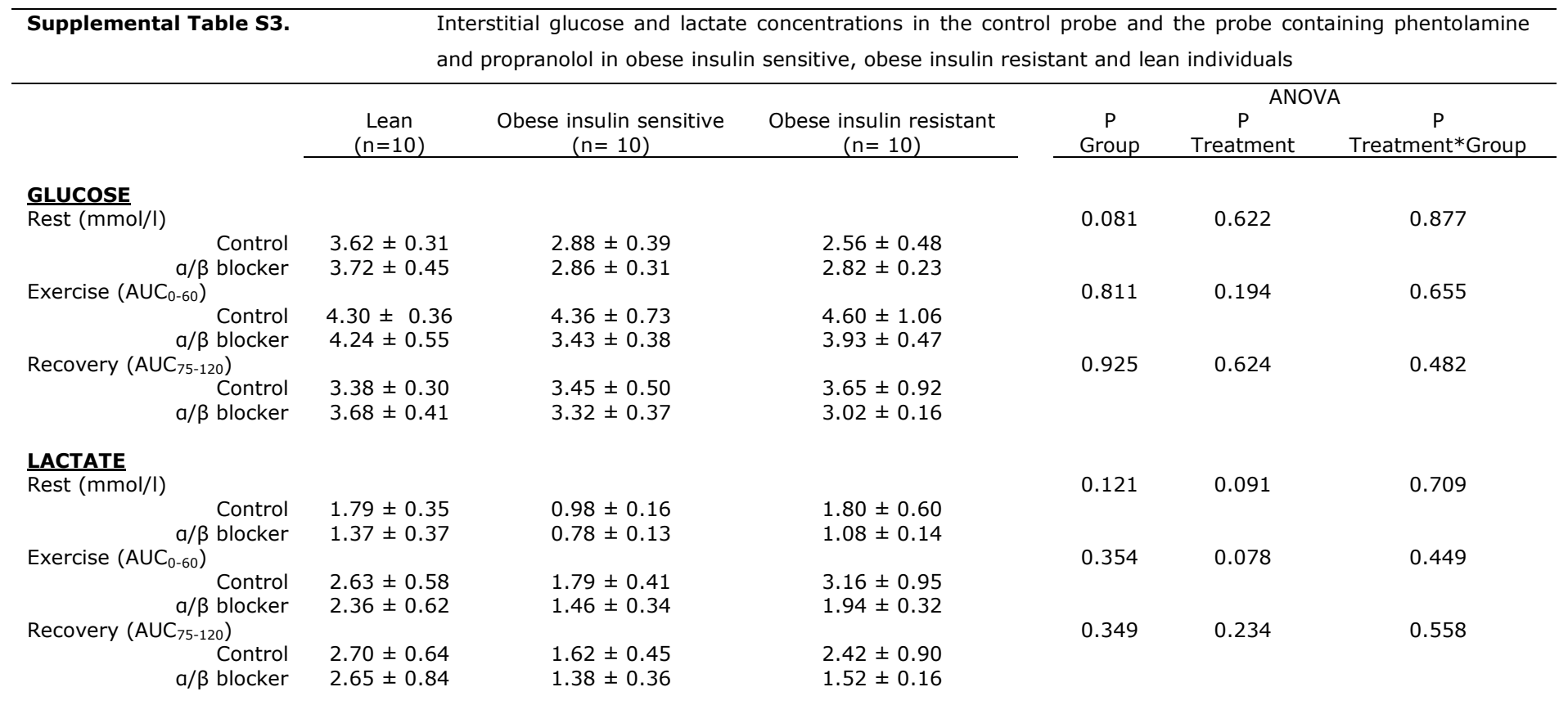

Data are mean \pm SEM. Control (probe containing Ringer), a/ $\beta$ blocker (probe containing Phentolamine + Propranolol).

$P$ value were calculated via a 2-way repeated ANOVA. 


\begin{tabular}{|c|c|c|c|c|c|c|c|}
\hline \multirow[t]{3}{*}{ Supplemental Table S4. } & \multicolumn{7}{|c|}{$\begin{array}{l}\text { Average concentrations of circulating glycerol, insulin, FFA, glucose, lactate, ANP, adrenalin and noradrenalin at rest, } \\
\text { during } 1 \mathrm{~h} \text { of exercise and } 1 \mathrm{~h} \text { of recovery, before and after } 12 \text { weeks of exercise training in obese insulin sensitive and } \\
\text { obese insulin resistant individuals }\end{array}$} \\
\hline & \multicolumn{2}{|c|}{ Obese insulin sensitive } & \multicolumn{2}{|c|}{ Obese insulin resistant } & \multicolumn{3}{|c|}{ ANOVA } \\
\hline & Pre & Post & Pre & Post & P Time & P Group & P Time*Group \\
\hline \multicolumn{8}{|l|}{ GLYCEROL } \\
\hline Rest $(\mu \mathrm{mol} / \mathrm{I})$ & $74.0 \pm 5.0$ & $64.2 \pm 4.8$ & $79.9 \pm 5.5$ & $74.8 \pm 6.4$ & 0.100 & 0.235 & 0.593 \\
\hline Exercise $\left(\mathrm{AUC}_{0-60}\right)\left(\mu \mathrm{mol} /{ }^{*} 60 \mathrm{~min}\right)$ & $157.3 \pm 22.7$ & $150.3 \pm 12.7$ & $175.7 \pm 24.1$ & $144.0 \pm 23.6$ & 0.026 & 0.730 & 0.500 \\
\hline Recovery $\left(A^{\prime} C_{75-105}\right)(\mu \mathrm{mol} / 1 * 45 \mathrm{~min})$ & $118.9 \pm 12.7$ & $124.4 \pm 6.6$ & $164.3 \pm 30.7$ & $122.1 \pm 19.3$ & 0.316 & 0.405 & 0.198 \\
\hline \multicolumn{8}{|l|}{ INSULIN } \\
\hline 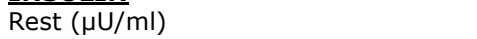 & $9.5 \pm 1.4$ & $9.4 \pm 1.3$ & $16.9 \pm 2.0$ & $14.3 \pm 1.3$ & 0.196 & 0.008 & 0.263 \\
\hline Exercise $\left(\mathrm{AUC}_{0-60}\right)(\mu \mathrm{U} / \mathrm{ml} * 60 \mathrm{~min})$ & $9.2 \pm 1.5$ & $9.1 \pm 1.2$ & $14.8 \pm 1.7$ & $13.4 \pm 1.3$ & 0.313 & 0.021 & 0.393 \\
\hline FFA & $11.6 \pm 1.6$ & $13.8 \pm 1.5$ & $21.6 \pm 1.5$ & $19.4 \pm 2.5$ & 0.981 & 0.003 & 0.206 \\
\hline Rest $(\mu \mathrm{mol} / \mathrm{l})$ & $506 \pm 48$ & $382 \pm 30$ & $571 \pm 37$ & $476 \pm 41$ & 0.016 & 0.060 & 0.723 \\
\hline Exercise $\left(\mathrm{AUC}_{0-60}\right)\left(\mu \mathrm{mol} / \mathrm{I}^{* 60 \mathrm{~min}}\right)$ & $533 \pm 55$ & $436 \pm 42$ & $540 \pm 51$ & $439 \pm 47$ & 0.040 & 0.930 & 0.954 \\
\hline $\begin{array}{l}\text { Recovery }\left(A \cup C_{75-105}\right)(\mu \mathrm{mol} / 1 * 45 \mathrm{~min}) \\
\text { GLUCOSE }\end{array}$ & $759 \pm 70$ & $698 \pm 38$ & $892 \pm 86$ & $726 \pm 76$ & 0.034 & 0.401 & 0.296 \\
\hline 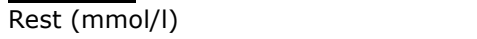 & $5.0 \pm 0.1$ & $5.3 \pm 0.2$ & $5.1 \pm 0.1$ & $5.1 \pm 0.1$ & 0.030 & 0.671 & 0.021 \\
\hline Exercise $\left(\mathrm{AUC}_{0-60}\right)\left(\mathrm{mmol} / \mathrm{I}^{* 60 \mathrm{~min})}\right.$ & $5.2 \pm 0.1$ & $5.3 \pm 0.1$ & $5.3 \pm 0.2$ & $5.1 \pm 0.1$ & 0.973 & 0.790 & 0.202 \\
\hline $\begin{array}{l}\text { Recovery }\left(\text { AUC }_{75-105}\right)(\mathrm{mmol} / \mathrm{I} * 45 \mathrm{~min}) \\
\text { LACTATE }\end{array}$ & $5.4 \pm 0.3$ & $5.4 \pm 0.1$ & $5.3 \pm 0.2$ & $5.1 \pm 0.1$ & 0.564 & 0.437 & 0.564 \\
\hline Rest $(\mathrm{mmol} / \mathrm{l})$ & $0.9 \pm 0.0$ & $0.8 \pm 0.1$ & $1.0 \pm 0.1$ & $1.0 \pm 0.1$ & 0.335 & 0.322 & 0.824 \\
\hline Exercise $\left(\mathrm{AUC}_{0-60}\right)\left(\mathrm{mmol} / I^{*} 60 \mathrm{~min}\right)$ & $1.1 \pm 0.1$ & $0.9 \pm 0.1$ & $1.4 \pm 0.1$ & $1.0 \pm 0.1$ & 0.016 & 0.127 & 0.609 \\
\hline $\begin{array}{l}\text { Recovery }\left(\mathrm{AUC}_{75-105}\right)\left(\mathrm{mmol} / \mathrm{I}^{*} 45 \mathrm{~min}\right) \\
\text { ANP }\end{array}$ & $1.0 \pm 0.1$ & $0.9 \pm 0.1$ & $1.2 \pm 0.1$ & $1.0 \pm 0.1$ & 0.078 & 0.392 & 0.606 \\
\hline $\operatorname{Rest}(\mathrm{pg} / \mathrm{ml})$ & $39.7 \pm 3.5$ & $33.8 \pm 3.6$ & $38.8 \pm 3.5$ & $37.5 \pm 3.5$ & 0.042 & 0.769 & 0.162 \\
\hline Exercise $\left(A^{\prime} C_{0-60}\right)(p g / m l * 60 m i n)$ & $44.6 \pm 4.7$ & $38.1 \pm 5.2$ & $41.1 \pm 2.5$ & $39.1 \pm 3.5$ & 0.043 & 0.819 & 0.256 \\
\hline Recovery $\left(A \cup C_{75-105}\right)(\mathrm{pg} / \mathrm{ml} * 45 \mathrm{~min})$ & $39.9 \pm 2.8$ & $36.2 \pm 5.0$ & $39.2 \pm 3.0$ & $37.6 \pm 3.8$ & 0.105 & 0.946 & 0.530 \\
\hline \multicolumn{8}{|l|}{ ADRENALIN } \\
\hline $\operatorname{Rest}(\mathrm{pg} / \mathrm{ml})$ & $29.4 \pm 6.8$ & $15.8 \pm 2.5$ & $21.9 \pm 5.6$ & $19.7 \pm 5.4$ & 0.155 & 0.773 & 0.295 \\
\hline Exercise $\left(A C_{0-60}\right)(\mathrm{pg} / \mathrm{ml} * 60 \mathrm{~min})$ & $85.6 \pm 7.1$ & $62.9 \pm 10.4$ & $58.0 \pm 9.2$ & $48.1 \pm 7.2$ & 0.029 & 0.057 & 0.354 \\
\hline 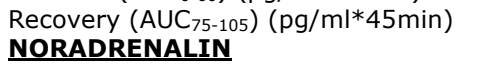 & $55.0 \pm 6.9$ & $36.6 \pm 5.0$ & $36.2 \pm 6.7$ & $24.4 \pm 3.6$ & 0.007 & 0.045 & 0.483 \\
\hline Rest $(\mathrm{pg} / \mathrm{ml})$ & $452 \pm 118$ & $395 \pm 28$ & $442 \pm 45$ & $400 \pm 41$ & 0.468 & 0.973 & 0.912 \\
\hline Exercise $\left(\mathrm{AUC}_{0-60}\right)(\mathrm{pg} / \mathrm{ml} * 60 \mathrm{~min})$ & $1078 \pm 92$ & $1002 \pm 93$ & $1043 \pm 118$ & $1045 \pm 106$ & 0.875 & 0.822 & 0.534 \\
\hline Recovery $\left(A \cup C_{75-105}\right)(\mathrm{pg} / \mathrm{ml} * 45 \mathrm{~min})$ & $519 \pm 50$ & $552 \pm 68$ & $584 \pm 63$ & $608 \pm 56$ & 0.613 & 0.377 & 0.930 \\
\hline
\end{tabular}

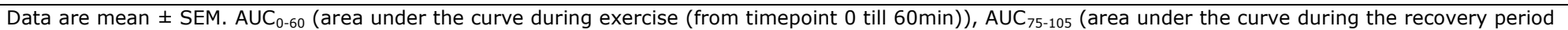
(from timepoint 75 until 105min)). Group effects were tested with a one-way ANOVA with Bonferroni post-hoc. 



\section{Chapter 6}

\section{Adipose tissue lipolytic inhibition}

\section{enhances the glucoregulatory properties}

of exercise in type 2 diabetes patients

Kenneth Verboven, Dominique Hansen, Jan-Willem van Dijk, Antoine Zorenc, Lennert Minten, Kevin Smeets, Lex B Verdijk, Luc JC van Loon 


\section{Abstract}

Aim - Exercise combined with adipose tissue lipolytic inhibition augments intramuscular lipid and glycogen use in type 2 diabetes mellitus (T2DM) patients. The present study investigates the impact of adipose tissue lipolytic inhibition during exercise on subsequent postprandial glycemic control in T2DM patients.

Methods - Fourteen male T2DM patients (age: $65 \pm 2$ years, $\mathrm{HbA}_{1 \mathrm{c}} 6.7 \pm 0.1 \%$ ) participated in a double-blind placebo-controlled randomized cross-over study in which subjects performed $60 \mathrm{~min}$ of endurance-type exercise (at $45 \% \mathrm{~W}_{\text {peak }}$ ) after being administered $250 \mathrm{mg}$ of an nicotinic acid analog (acipimox; ACP) or a placebo (PLA). A control experiment was included in which no exercise was performed (CON). Plasma samples were obtained before, during and for up to $7.5 \mathrm{~h}$ after exercise.

Results - Sixty min of exercise at $73 \pm 6 \mathrm{~W}$ did not significantly lower circulating plasma glucose and insulin excursions in PLA when compared with CON $(P=0.300$ and 0.778 for glucose and insulin, respectively). Acipimox administration strongly reduced circulating plasma FFA concentrations during exercise ( $175 \pm 13$ vs $535 \pm 47$ and $637 \pm 58 \mathrm{mmol} / \mathrm{L}$ in the ACP vs CON and PLA, respectively; $\mathrm{P}<0.001$ ). Circulating plasma glucose (tAUC, $3500 \pm 124$ vs $3794 \pm 172$ and $3946 \pm 183 \mathrm{mmol} / \mathrm{L} / 450 \mathrm{~min}$ ) and insulin (tAUC, 76 \pm 7 vs $106 \pm 13$ and $103 \pm 13 \mathrm{nmol} / \mathrm{L} / 450 \mathrm{~min}$ ) excursions were substantially lower during $7.5 \mathrm{~h}$ of recovery from exercise (i.e. postprandially) in ACP when compared with either CON or PLA ( $P<0.05$ and $<0.001$, respectively).

Conclusions - Exercise with adipose tissue lipolytic inhibition improves postprandial blood glucose regulation and thus clinical efficacy in male T2DM patients. 


\section{Introduction}

In the treatment of type 2 diabetes mellitus (T2DM), tight glycemic control is important to reduce the likelihood of developing cardiovascular disease or other diabetes-related complications ${ }^{1,2}$. Despite the use of blood glucose-lowering medication, postprandial blood glucose excursions remain prevalent throughout the day in most T2DM patients ${ }^{3}$. Postprandial hyperglycemia contributes substantially to the glycation of hemoglobin ${ }^{4}$ and forms an early sign of metabolic abnormalities before developing T2DM ${ }^{5}$. Adjuvant strategies are warranted to attenuate blood glucose excursions throughout the day and to improve glycemic control in T2DM patients ${ }^{6}$. Along with proper dietary counseling and blood glucose-lowering medication, exercise forms another cornerstone in T2DM treatment 7,8 . It has been postulated that the glucoregulatory properties of each individual exercise session are of key importance to achieving long-term glycemic control targets ${ }^{9}$. A single bout of exercise has been shown to enhance insulin sensitivity and improve glycemic control for up to $48 \mathrm{~h}^{10-12}$. Despite the use of different treatment strategies, many T2DM patients do not meet glycemic targets ${ }^{8}$ and as these patients mostly suffer from poor exercise tolerance ${ }^{7}$, we need to develop strategies that can augment the glucoregulatory properties of exercise and exercise training ${ }^{13}$ in order to further improve the clinical efficacy of these treatment strategies.

Disturbances in fatty acid handling play an essential role in the development or progression of peripheral insulin resistance in T2DM ${ }^{14}$. Impairments in the buffering capacity of the adipose tissue ${ }^{14}$, as well as skeletal muscle lipid mobilization and oxidation ${ }^{15,16}$ contribute to elevated plasma free fatty acids (FFA). Excess availability of plasma derived FFA results in ectopic lipid deposition in insulin sensitive tissues such as liver, pancreas, heart and skeletal muscle tissue ${ }^{14}$. These metabolic disturbances cause a state of metabolic inflexibility ${ }^{17}$, further promoting lipid deposition in skeletal muscle as opposed to their mobilization and oxidation, thereby increasing peripheral insulin resistance ${ }^{18}$.

One of the mechanisms by which exercise is believed to improve insulin sensitivity is the mobilization and oxidation of the intramuscular lipid depots ${ }^{19}$, which is largely determined by circulating plasma FFA concentrations ${ }^{20,21}$. The 
elevated plasma FFA concentrations in obese or T2DM patients inhibit the hydrolysis of intramuscular lipid stores ${ }^{21}$. Of interest, administration of the nicotinic acid analog acipimox has previously been shown to increase intramuscular triglyceride use during exercise in T2DM patients, reduce intramuscular lipid contents and lower circulating insulin concentrations in T2DM patients ${ }^{21}$. We hypothesize that adipose tissue lipolytic inhibition during endurance-type exercise will help to further improve postprandial glycemic control in T2DM patients. To test our hypothesis, we determined the impact of a single bout of moderate-intense endurance-type exercise with or without adipose tissue lipolysis inhibition (acipimox administration) on subsequent postprandial blood glucose and insulin excursions throughout the day in male T2DM patients. 


\section{Methods}

Subjects - A total of 18 male T2DM patients on blood glucose-lowering medication were selected to participate in this study. Patients were included based upon the following inclusion criteria: blood glycated hemoglobin (HbA1c) $>6.5 \%$ (>48 mmol $/ \mathrm{mol}$ ), aged 45-75 y, body mass index (BMI) 27.5-35.0 $\mathrm{kg} / \mathrm{m}^{2}$, sedentary lifestyle ( $<2 \mathrm{~h}$ sports related activities per week) and Caucasian ethnicity. Exclusion criteria were: exogenous insulin therapy, selfreported coronary artery, pulmonary, renal or gastric disease, orthopedic symptoms that would interfere with exercise, or involvement in an exercise training or caloric restriction program within one year prior to the current study. Due to lack of motivation, four subjects withdrew from the study, leaving 14 subjects (Supplemental Fig. 1). This study was approved by the local medical ethical committee (Jessa Hospital and Hasselt University, Hasselt, Belgium), and the study was performed conform to the standards set by the latest revision (2013) of the Declaration of Helsinki. After careful explanation about the nature and risks of the experimental procedures, all subjects gave their written informed consent before participating in the study (study registration number NTR4710).

Screening and testing - Following a 2-day cessation of blood-glucose lowering medication intake, subjects arrived at the laboratory at 08.00 AM after an overnight fast. A fasting blood sample was obtained for blood HbA1c concentration (Hi-Auto A1c Analyzer, Menarini Diagnostics, Florence, Italy). Subjects underwent an evaluation of body composition by a dual x-ray absorptiometry scan (DEXA, Lunar DPXL, WI, USA), followed by a maximal cardiopulmonary exercise test on a cycle ergometer (eBike Basic, General Electric $\mathrm{GmbH}$, Bitz, Germany) to assess peak oxygen uptake capacity (VO2peak) and workload capacity (Wpeak), using a 1-min work stage protocol (starting workload of $40 \mathrm{~W}$, incremental workload of $20 \mathrm{~W}$ ). VO2 measurements were performed continuously (Jaeger Oxycon, Erich Jaeger $\mathrm{GmbH}$, Germany), and VO2peak was compared with normal age- and gender-related VO2peak (expressed as \%VO2peak predicted) ${ }^{22}$. Heart rate (HR) was monitored continuously using a 12-leads electrocardiogram. All subjects cycled until exhaustion. The test was ended when subjects were no longer able to maintain a 
cycling frequency of $55 \mathrm{rpm}$ or higher. Peak exercise effort was confirmed when respiratory gas exchange ratio (RER) was $\geq 1.10$, in combination with dyspnea, leg and/or general fatigue.

Study design - Subjects participated in a randomized (double-blind, placebocontrolled) cross-over trial consisting of three conditions, interspersed by one week between treatments. Subjects were randomly assigned (Supplemental Fig. 1) to either a non-exercise condition (no exercise with placebo intake, CON), or to conditions in which a 60-min endurance-type exercise bout was performed exactly 60 min after per os administration of a nicotinic acid derivate (acipimox, ACP) or a placebo (PLA). Subjects were subsequently followed for $7.5 \mathrm{~h}$ in the laboratory. Twenty-two hours after cessation of exercise subjects returned to the laboratory for an oral glucose tolerance test (OGTT) (Fig. 1).

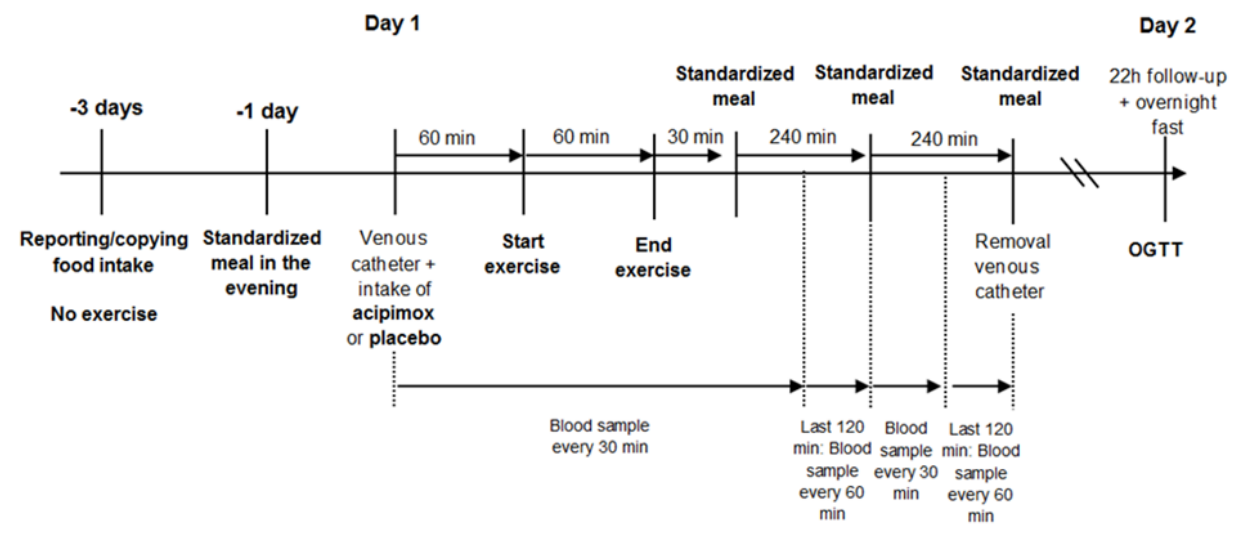

Figure 1 - Study design. Outline of the study protocol. On day 1 , following administration of a placebo or the nicotinic acid analog acipimox, resting blood samples were collected for $60 \mathrm{~min}$. In the three treatments (i.e. control condition, placebo intake with exercise and acipimox intake with exercise), fasting blood samples were collected at rest, during exercise and post-exercise at different time points for a total duration of $10.5 \mathrm{~h}$. Twenty-two hours after cessation of exercise an oral glucose tolerance test was performed. 
Study protocol - On day 1 of each intervention period, subjects arrived at the laboratory at 08.00AM following an overnight fast. An intravenous catheter was inserted in an antecubital vein for blood sampling purposes, one hour later followed by the oral administration of a placebo capsule (gelatin capsule containing starch) or a capsule of identical size and color containing $250 \mathrm{mg}$ of the nicotinic acid analog acipimox (Nedios, Altana Pharma, Hoofddorp, The Netherlands). Except for flushing, no side-effects were experienced by the subjects after ACP administration. One hour after placebo or acipimox administration, subjects performed $60 \mathrm{~min}$ of endurance-type exercise on an electronically braked bike at $45 \%$ of their individual Wpeak (PLA or ACP) or remained sedentary (CON). Heart rate (HR) was monitored continuously (Polar, Oy, Finland). Energy expenditure was calculated by use of ACSM equations ${ }^{23}$.

Subjects next consumed three standardized meals during the day in the laboratory at $30 \mathrm{~min}$ (total energy: $2203 \mathrm{~kJ}$ (545 kcal); $18 \mathrm{~g}$ fat; $64 \mathrm{~g}$ carbohydrates; $25 \mathrm{~g}$ protein), $270 \mathrm{~min}$ (total energy: $2480 \mathrm{~kJ}$ (595 kcal); $36 \mathrm{~g}$ fat; $36 \mathrm{~g}$ carbohydrates; $27 \mathrm{~g}$ protein) and $510 \mathrm{~min}$ (total energy: $3292 \mathrm{~kJ}$ (788 $\mathrm{kcal}) ; 42 \mathrm{~g}$ fat; $71 \mathrm{~g}$ carbohydrates; $27 \mathrm{~g}$ protein) after cessation of exercise with ad libitum water consumption. Venous blood samples were collected every 30 or 60 min after consumption of the first and second standardized meal in EDTA-containing tubes and immediately centrifuged at $1,000 \mathrm{~g}$ for $5 \mathrm{~min}$ at $4^{\circ} \mathrm{C}$. Plasma aliquots were frozen in liquid nitrogen and stored at $-80^{\circ} \mathrm{C}$ until analysis. On day 2, subjects returned to the laboratory at $08.00 \mathrm{AM}$ following an overnight fast ( $\geq 10 \mathrm{~h}$ ) for an OGTT (starting at $22 \mathrm{~h}$ after completion of the exercise bout), in which blood glucose and insulin concentrations were assessed every $30 \mathrm{~min}$.

Blood chemistry - Plasma glucose (A11A01667, Cobas Pentra semiautomatic analyzer, Roche, Basel, Switzerland), insulin (HI-14K, Human Insulin specific RIA Kit, Millipore, Billerica, MA, USA), FFA (NEFA HR (2) R1 set 434-91795 and Nefa HR (2) R2 set 436-91995 Wako, Neuss, Germany), triglyceride (A11A01640 (Roche), ABX Pentra Triglycerides CP, HORIBA ABX), and lactate (NAD 10127990001 and LDH 10127876001, Cobas Pentra semiautomatic analyzer, Roche, Basel, Switzerland) concentrations were assessed in duplo in each sample. 
Medication, food intake and habitual physical activity - Except for screening, medication intake remained unchanged during the entire study period and was taken on the morning of each experimental visit (during the first standardized meal on day 1 and prior to the OGTT on day 2). Subjects maintained their normal habitual physical activity level and diet, but refrained from exhaustive physical activity three days prior to each visit. In addition, subjects recorded their dietary intake over three days prior to day 1 and copied their diet prior to each subsequent visit.

Statistical analysis - Data are presented as means \pm SEM. Shapiro-Wilk test indicated no normal distribution of data. To compare blood parameters (interval data) between different conditions, Friedman tests (for three related samples) were performed. In case of a significant main effect, post-hoc Wilcoxon signed ranks tests (for two related samples) were performed corrected for multiple testing (Bonferroni). Total areas under the curve (tAUC) of these blood parameters was calculated by the trapezoid method before and during exercise (08.00-10.30 AM; tAUC $_{0-150)}$ and for a total of $7.5 \mathrm{~h}$ after exercise (11.00 AM 06.30 PM; tAUC $180-630$ ). In addition, tAUC during OGTT on day 2 was calculated. Statistical significance was set at $P<0.05$ (two-tailed) for main effect and $P<0.017$ (two-tailed) for two related samples. SPSS 22 for Windows was used to perform all calculations (IBM Corporation, Armonk, NY, USA). 


\section{Results}

\section{Subjects' characteristics}

Fourteen male T2DM patients (age: $65 \pm 2 \mathrm{y}, \mathrm{HbA}_{1 \mathrm{c}} 6.7 \pm 0.1 \%(50 \pm 2 \mathrm{mmol} / \mathrm{mol}$ ), mean disease duration $10 \pm 2$ y) participated in this study. All subjects were either overweight or obese (BMI $30.2 \pm 0.9 \mathrm{~kg}^{*}\left(\mathrm{~m}^{2}\right)^{-1}$, whole-body fat $31 \pm 1 \%$ ), showed a relative low exercise tolerance $\left(\mathrm{VO}_{2 \text { peak }} 36 \pm 2 \mathrm{~mL}^{*} \mathrm{~min}^{-1} * \mathrm{~kg} \mathrm{FFM}^{-1}\right.$ or $78 \pm 4 \%$ of predicted norm) and maximal cycling power output (absolute $\mathrm{W}_{\text {peak }}$ $162 \pm 12 \mathrm{~W}$, relative $W_{\text {peak }} 2.7 \pm 0.1 \mathrm{~W}^{*} \mathrm{~kg} \mathrm{FFM}^{-1}$ ). Except for one patient on monotherapy (DPP-4 inhibitor), all patients were treated with metformin $(n=11)$ and/or other treatments including lipid lowering $(n=7)$, antiplatelet $(n=6)$, blood pressure lowering $(n=7)$ or additional glucose lowering $(n=8)$ therapy. Subjects' characteristics are summarized in Table 1.

Table 1 Subjects' characteristics

\begin{tabular}{|c|c|c|c|}
\hline Variable & & \multicolumn{2}{|l|}{ Medication use } \\
\hline$n$ & 14 & & \\
\hline Age, years & $65 \pm 2$ & Metformin $(n)$ & 11 \\
\hline BMI, $k g^{*}\left(m^{2}\right)^{-1}$ & $30.2 \pm 0.9$ & Sulfonylurea $(n)$ & 2 \\
\hline Body fat, \% & $31 \pm 1$ & DPP-4 inhibitor $(n)$ & 6 \\
\hline \multirow[t]{2}{*}{ Fat free mass, kg } & $60.0 \pm 2.1$ & GLP-1 receptor agonist $(n)$ & 1 \\
\hline & & Statin $(n)$ & 7 \\
\hline HbA1c, \% & $6.7 \pm 0.1$ & Antiplatelet $(n)$ & 6 \\
\hline $\mathrm{HbA} 1 \mathrm{c}, \mathrm{mmol} / \mathrm{mol}$ & $50 \pm 2$ & Calcium antagonist $(n)$ & 3 \\
\hline \multirow[t]{2}{*}{ Years since diagnosis } & $10 \pm 2$ & ACE inhibitor $(n)$ & 3 \\
\hline & & Angiotensin-2 receptor antagonist $(n)$ & 1 \\
\hline VO2 peak, $\mathrm{ml}^{*} \mathrm{~min}^{-1} * \mathrm{~kg}^{-1}(\mathrm{FFM})$ & $36.3 \pm 2.0$ & Diuretics $(n)$ & 1 \\
\hline Wmax, Watt*kg ${ }^{-1}$ (FFM) & $2.7 \pm 0.1$ & Beta blocker $(n)$ & 3 \\
\hline Maximal heart rate, beats* $\min ^{-1}$ & $144 \pm 4$ & & \\
\hline Maximal RER & $1.17 \pm 0.01$ & & \\
\hline
\end{tabular}

\section{Plasma FFA and triglycerides}

All subjects performed two separate 60 -min cycling exercise bouts at $45 \%$ of their $\mathrm{W}_{\text {peak }}(73 \pm 6 \mathrm{~W})$, eliciting a HR of $112 \pm 4$ and $116 \pm 4$ bts $^{*} \mathrm{~min}^{-1}$ in PLA and $A C P$, respectively $(P>0.05)$. Energy expenditure during both exercise bouts was 
estimated (heart rate based) to be $1.4 \pm 0.1 \mathrm{MJ}$. In the fasted state, plasma FFA concentrations increased in both CON and PLA over time ( $P=0.001$ and $P=0.001$, respectively), with a greater increase following the onset of exercise (PLA) when compared with $\operatorname{CON}(P=0.022)$ (Fig. $2 A)$. Acipimox administration prevented the fasting- (CON) and exercise-induced (PLA) rise in circulating plasma FFA concentrations, resulting in lower plasma FFA concentrations when compared with both CON and PLA ( $P<0.001$ ) (Fig. $2 A$ ). Overall tAUC $_{0-150}$ for the plasma FFA concentrations differed significantly between treatments $\left(P_{\text {treatment }}<0.001\right)$ and averaged $79 \pm 7 \mathrm{mmol}^{-1} * 150 \mathrm{~min}^{-1}, 96 \pm 8 \mathrm{mmol}^{*} \mathrm{~L}^{-1} * 150 \mathrm{~min}^{-1}$ and $40 \pm 2$ $\mathrm{mmol} * \mathrm{~L}^{-1} * 150 \mathrm{~min}^{-1}$ in CON, PLA and ACP, respectively (Table 2 ). After exercise, plasma FFA concentrations remained significantly lower in ACP when compared with both CON and PLA (Fig. 2A), whereby overall tAUC ${ }_{180-630}$ for the postprandial plasma FFA concentrations differed significantly between treatments ( $P_{\text {treatment }}=0.002$ ) and averaged $149 \pm 15 \mathrm{mmol}^{*} \mathrm{~L}^{-1} * 450 \mathrm{~min}^{-1}$, $170 \pm 13 \mathrm{mmol}^{*} \mathrm{~L}^{-1} * 450 \mathrm{~min}^{-1}$ and $115 \pm 16 \mathrm{mmol}^{*} \mathrm{~L}^{-1} * 450 \mathrm{~min}^{-1}$ in CON, PLA and $A C P$, respectively (Table 2 ). Plasma triglyceride concentrations slightly increased over time and did not show any differences between treatments in the fasted $\left(P_{\text {treatment }}=0.789\right)$ or postprandial state $\left(P_{\text {treatment }}=0.458\right)($ Fig. $2 B$; Table 2$)$.

\section{Plasma glucose and insulin}

ACP did not affect exercise-induced plasma lactate levels when compared with PLA ( $P=0.683)$, while plasma lactate concentrations were higher during exercise in PLA $\left(3.14 \pm 0.30 \mathrm{mmol} * \mathrm{~L}^{-1}, \quad \mathrm{P}=0.001\right)$ and ACP $\left(3.02 \pm 0.23 \mathrm{mmol} * \mathrm{~L}^{-1}\right.$, $\mathrm{P}=0.001)$ treatments when compared with $\mathrm{CON}\left(1.14 \pm 0.10 \mathrm{mmol}^{*} \mathrm{~L}^{-1}\right)$ (Fig. $3 \mathrm{C}$ ). Postprandial plasma lactate concentrations did not differ between treatments $\left(P_{\text {treatment }}=0.395\right)$ (Fig. 3C). Plasma glucose concentrations (Fig. 3A) and $\mathrm{tAUC}_{0-}$ 150 (Table 2) were not significantly different between treatments during exercise $\left(P_{\text {treatment }}=0.607\right.$ and $P_{\text {treatment }}=0.607$, respectively $)$ Plasma insulin concentrations (Fig. 3B) and $\mathrm{tAUC}_{0-150}$ (Table 2) were not significantly different between treatments during exercise $\left(P_{\text {treatment }}=0.751\right.$ and $P_{\text {treament }}=0.223$, respectively). 


\begin{tabular}{|c|c|c|c|c|}
\hline \multirow[b]{2}{*}{ Fasting and during exercise } & \multicolumn{4}{|c|}{ Treatment } \\
\hline & $\begin{array}{l}\text { Control } \\
\text { (no exercise } \\
+ \text { placebo) } \\
\end{array}$ & $\begin{array}{c}\text { Exercise+ } \\
\text { placebo }\end{array}$ & $\begin{array}{l}\text { Exercise+ } \\
\text { acipimox }\end{array}$ & Ptreatment \\
\hline tAUC glucose (mmol/L/150min) & $1094 \pm 56$ & $1119 \pm 65$ & $1096 \pm 61$ & 0.607 \\
\hline tAUC insulin (nmol/L/150min) & $11 \pm 0.9$ & $11 \pm 1$ & $10 \pm 1$ & 0.223 \\
\hline tAUC lactate (mmol/L/150min) & $177 \pm 16$ & $314 \pm 30 *$ & $305 \pm 20 *$ & $<0.001$ \\
\hline $\begin{array}{l}\text { tAUC triglycerides (mmol/L/150min) } \\
\text { tAUC free fatty acids } \\
\text { (mmol/L/150min) }\end{array}$ & $\begin{array}{c}210 \pm 36 \\
80 \pm 7\end{array}$ & $207 \pm 28$ & $217 \pm 38$ & $<0.001$ \\
\hline \multicolumn{5}{|l|}{ Postprandial during the day } \\
\hline tAUC glucose (mmol/L/450min) & $3794 \pm 172$ & $3946 \pm 183$ & $3500 \pm 124 \#$ & 0.011 \\
\hline peak glucose (mmol/L) & $11.7 \pm 0.6$ & $12.2 \pm 0.6$ & $11.1 \pm 0.6$ & 0.526 \\
\hline tAUC insulin (nmol/L/450min) & $106 \pm 13$ & $103 \pm 13$ & $76 \pm 7 * \#$ & $<0.001$ \\
\hline peak insulin (pmol/L) & $414 \pm 93$ & $388 \pm 88$ & $263 \pm 36 * \#$ & 0.013 \\
\hline tAUC lactate (mmol/L/450min) & $827 \pm 101$ & $841 \pm 77$ & $790 \pm 73$ & 0.395 \\
\hline $\begin{array}{l}\text { tAUC triglycerides }(\mathrm{mmol} / \mathrm{L} / 450 \mathrm{~min}) \\
\text { tAUC free fatty acids } \\
\text { (mmol/L/450min) }\end{array}$ & $\begin{array}{l}858 \pm 134 \\
149 \pm 16\end{array}$ & $817 \pm 94$ & $800 \pm 126$ & 0.458 \\
\hline \multicolumn{5}{|l|}{ During OGTT } \\
\hline tAUC glucose (mmol/L/120min) & $1586 \pm 83$ & $1577 \pm 93$ & $1580 \pm 86$ & 0.607 \\
\hline peak glucose (mmol/L) & $15.6 \pm 0.8$ & $15.5 \pm 0.9$ & $15.7 \pm 0.8$ & 0.807 \\
\hline tAUC insulin (nmol/L/120min) & $31 \pm 2$ & $30 \pm 3$ & $31 \pm 4$ & 0.931 \\
\hline peak insulin $(\mathrm{pmol} / \mathrm{L})$ & $361 \pm 41$ & $347 \pm 41$ & $368 \pm 55$ & 0.807 \\
\hline
\end{tabular}

Data are expressed as means \pm SEM. *Significantly different as opposed to control condition $(p<0.017)$. \#Significantly different as opposed to exercise with placebo intake $(p<0.017)$. tAUC, total area under the curve; OGTT, oral glucose tolerance test.

Following exercise, postprandial plasma glucose concentrations were substantially lower in ACP when compared with PLA ( $P_{\text {treatment }}=0.011$ ). This resulted in overall significantly different plasma glucose tAUC $_{180-630}$ between treatments, with lower plasma glucose concentrations in ACP when compared with PLA ( $P=0.009)$ and a strong trend for lower plasma glucose concentrations in ACP when compared to CON $(P=0.041)$ (Table 2; Fig. 4). tAUC $_{180-630}$ averaged $3794 \pm 172 \mathrm{mmol}^{*} \mathrm{~L}^{-1} * 450 \mathrm{~min}^{-1}, 3946 \pm 183 \mathrm{mmol}^{-1} * 450 \mathrm{~min}^{-1}$ and $3500 \pm 124$ mmol*L ${ }^{-1} * 450 \mathrm{~min}^{-1}$ in $\mathrm{CON}, \mathrm{PLA}$ and $\mathrm{ACP}$, respectively, while postprandial peak plasma glucose concentrations (measured during the entire postprandial timeframe) did not differ between treatments $\left(P_{\text {treatment }}=0.526\right)$ (Table 2). Following exercise, ACP substantially reduced postprandial plasma insulin 
excursions when compared with $\operatorname{CON}(P=0.002)$ or PLA $(P=0.001)$ $\left(P_{\text {treatment }}<0.001\right)$. These reductions resulted in overall significantly lower insulin tAUC $_{180-630}$ between treatments (Table 2; Fig. 4). Hence, tAUC $180-630$ averaged $106 \pm 13 \mathrm{nmol} * \mathrm{~L}^{-1} * 450 \mathrm{~min}^{-1}, 103 \pm 13 \mathrm{nmol} * \mathrm{~L}^{-1} * 450 \mathrm{~min}^{-1}$ and $76 \pm 7 \mathrm{nmol} * \mathrm{~L}^{-}$ $1 * 450 \mathrm{~min}^{-1}$ in CON, PLA and ACP, respectively (Table 2 ). In addition, postprandial peak plasma insulin concentrations (measured during the entire postprandial timeframe) were substantially reduced in ACP compared with CON and PLA ( $P_{\text {treatment }}=0.013$ ). Compared with CON (peak insulin: $414 \pm 93 \mathrm{pmol}^{*} \mathrm{~L}^{-1}$, $\mathrm{P}=0.005$ ) and PLA (peak insulin: $388 \pm 88$ pmol* $\mathrm{L}^{-1}, \mathrm{P}=0.022$ ), ACP significantly lowered postprandial peak insulin concentrations (peak insulin: $263 \pm 36 \mathrm{pmol}^{*} \mathrm{~L}^{-}$ ${ }^{1}$ ) (Fig. 2D; Table 2).

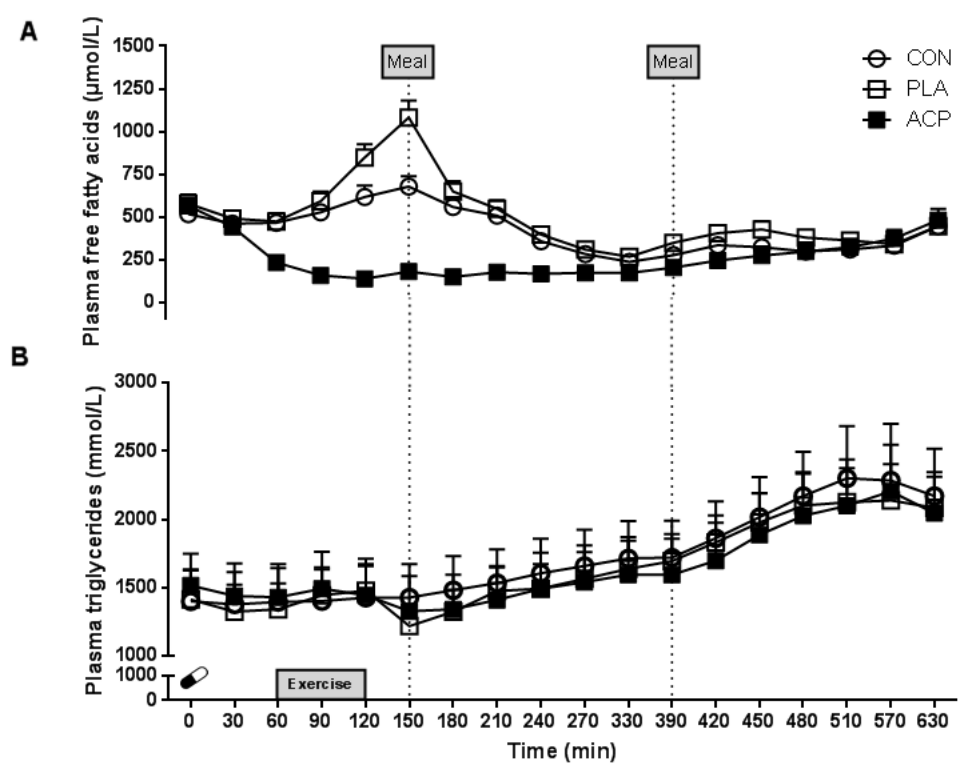

Figure 2 - Plasma metabolite concentrations. Data represent means + SEM; $n=14$. Plasma FFA (A) and plasma triglycerides (B) concentrations at rest, during endurance-type exercise (or the control condition) and in the subsequent postprandial state. 


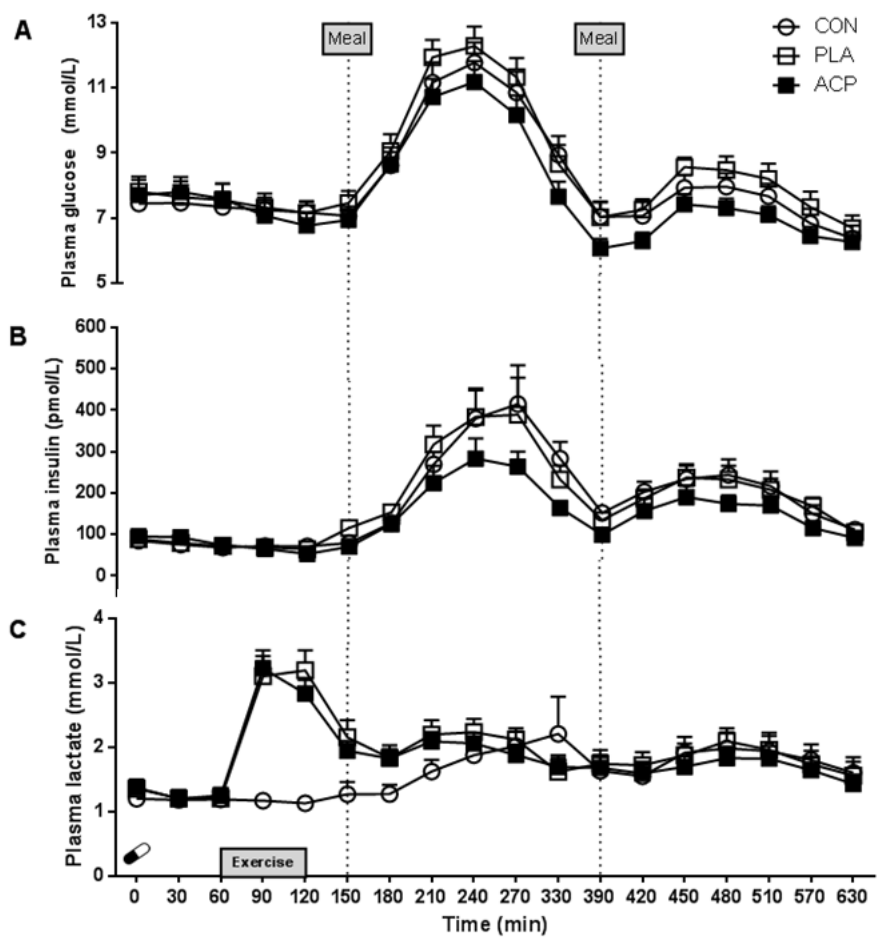

Figure 3 - Plasma metabolite concentrations. Data represent means + SEM; $n=14$. Plasma glucose (A), plasma insulin (B) and plasma lactate (C) concentrations at rest, during endurance-type exercise (or the control condition) and in the subsequent postprandial state.
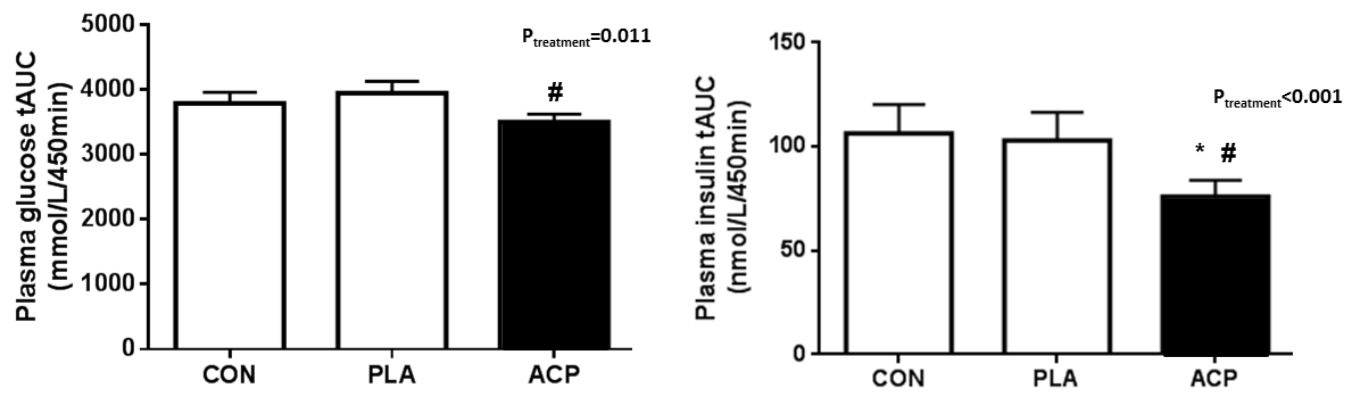

Figure 4 - Postprandial plasma glucose and plasma insulin responses expressed as total area under the curve. Data represent means $+\mathrm{SEM} ; n=14$. Plasma glucose and plasma insulin concentrations were determined in the postprandial phase following exercise for up to $7.5 \mathrm{~h}$ ( $\mathrm{tAUC}_{180-}$ 630). P-values represent treatment (main) effect. * Significantly different from control condition (CON) $(P<0.017)$; \# Significantly different from exercise with placebo intake $(P L A)(P<0.017)$. 


\section{Oral glucose tolerance test}

The OGTT performed $22 \mathrm{~h}$ after exercise showed no differences between CON, ACP and PLA with respect to fasting plasma glucose $(7.7 \pm 0.4,7.5 \pm 0.4$, and $7.9 \pm 0.3 \mathrm{mmol} * \mathrm{~L}^{-1}$, respectively; $\left.P_{\text {treatment }}=0.526\right)$ and fasting plasma insulin concentrations $\left(89.5 \pm 10.4,83.2 \pm 10.3\right.$, and $94.3 \pm 12.4 \mathrm{pmol}^{*} \mathrm{~L}^{-1}$, respectively; $\left.P_{\text {treatment }}=0.751\right)$, peak plasma glucose $(15.6 \pm 0.8,15.7 \pm 0.8$, and $15.5 \pm 0.9$ mmol* $\mathrm{L}^{-1}$, respectively; $\left.P_{\text {treatment }}=0.807\right)$ and insulin concentrations $(361 \pm 41$, $368 \pm 55$, and $347 \pm 41$ pmol $* \mathrm{~L}^{-1}$, respectively; $P_{\text {treatment }}=0.807$ ) or in their corresponding tAUC $_{0-120} \quad\left(P_{\text {treatment }}=0.607\right.$ and $P_{\text {treatment }}=0.931$, respectively $)$ between treatments (Table 2 ). 


\section{Discussion}

In the present study, we show that inhibiting adipose tissue lipolysis during a single bout of endurance-type exercise reduces plasma FFA availability in male T2DM patients. The lower plasma FFA concentrations during exercise were associated with a reduced postprandial rise in circulating blood glucose and insulin concentrations for at least 7.5 hours after cessation of exercise, when compared with exercise performed without adipose tissue lipolytic inhibition. These glucoregulatory benefits of combining exercise with adipose tissue lipolytic inhibition were no longer present 24 hours after cessation of exercise.

Patients with T2DM generally suffer from exercise intolerance and muscle weakness ${ }^{7}$. In line, our patients were sedentary and showed a relative low physical fitness upon medical screening (Table 1 ) as compared with agematched healthy individuals ${ }^{24}$. The applied $60 \mathrm{~min}$ bout of moderate-intensity exercise performed at $45 \% \mathrm{~W}_{\max }$ (which was in accordance to the most recent clinical guidelines for T2DM treatment ${ }^{8}$ ) represented an intense effort for these patients, but the absolute workload was merely $73 \pm 6 \mathrm{~W}$. Despite the effort, the exercise bout did not substantially improve glycemic control during exercise (PLA). Furthermore, postprandial glucose as well as insulin excursions throughout $7.5 \mathrm{~h}$ of recovery from exercise did not substantially differ from the non-exercise control trial (CON). Regardless of these somewhat unexpected findings, the current results clearly substantiate our opinion that new strategies that can help to augment the (clinical) benefits of exercise should be explored. Optimized therapies are warranted to further improve glycemic control in those patients that have been sedentary and are commencing a lifestyle intervention program.

Previous work has shown that the pool of intramuscular lipids are not mobilized during exercise when circulating plasma FFA concentrations are moderately elevated (exceeding 300-500 $\mu \mathrm{mol}^{*} \mathrm{~L}^{-1}$ ), especially in obese and type 2 diabetic individuals ${ }^{25}$. Using a nicotinic acid analog to specifically block adipose tissue lipolysis ${ }^{26}$, we previously showed that the exercise-induced increase in circulating plasma FFA concentrations can be prevented, thereby allowing the intramuscular lipid pool to be mobilized in both healthy athletes ${ }^{20}$ as well as 
T2DM patients ${ }^{21}$. In line, circulating plasma FFA concentrations during moderate-intense endurance-type exercise were successfully lowered by providing our patients with $250 \mathrm{mg}$ acipimox $60 \mathrm{~min}$ prior to this exercise bout. Accordingly, we hypothesize that under these conditions exercise allows both intramuscular lipid as well as glycogen pools to be mobilized $20,25,27$, thereby increasing peripheral insulin sensitivity and, as such, improving glycemic control 28 .

Exercise in combination with adipose tissue lipolytic inhibition (ACP) substantially reduced subsequent postprandial circulatory glucose and insulin responses for up to $7.5 \mathrm{~h}$ after cessation of exercise. A substantial decline in postprandial circulatory glucose $(-11 \pm 3 \%)$ and insulin $(-25 \pm 4 \%)$ concentrations were observed following ACP when compared to PLA (Fig. 4). This indicates that adipose tissue lipolytic inhibition during low- to moderate-intense exercise can strongly increase insulin action. This results in lower postprandial blood glucose excursions with less circulating insulin, making this type of combinational exercise as effective as exercise bouts of either higher intensity ${ }^{29}$ or longer duration ${ }^{30}$. Still, it remains unclear whether the volume of endurance exercise affects glycemic control, or whether the different exercise characteristics modulate the impact of exercise on glycemic control ${ }^{9}$. The benefits of exercise with adipose tissue lipolytic inhibition on glycemic control were evident for the remainder of the day after cessation of exercise, an important finding with respect to the importance of postprandial plasma glucose excursions in predicting secondary diabetes complications ${ }^{31}$. However, at $24 \mathrm{~h}$ after exercise, we no longer observed greater whole-body glucose tolerance in ACP or PLA treatments, as no differences in circulatory plasma glucose or insulin responses were observed following the OGTT (Table 2). Therefore, the need for structured exercise or increased physical activity on a daily basis should be emphasized in this population.

In the present study, combining moderate-intense endurance-type exercise with adipose tissue lipolytic inhibition (ACP) was shown to improve postprandial glycemic control more effectively when compared with both the exercise only treatment (PLA) as well as the non-exercise control treatment (CON). Thus, combining exercise with adipose tissue lipolytic inhibition is postulated to be a 
more effective interventional strategy to augment the glucoregulatory benefits of exercise intervention in T2DM treatment. Opposed to the combination therapy, one could suggest that adipose tissue lipolytic inhibition might be used without implementing exercise. However, the chronic use of nicotinic acid (or its analogs) is contraindicated. Previous work has shown that more prolonged use of acipimox can eventually lead to tolerance development as indicated by unaffected or even increased circulatory plasma FFA concentrations ${ }^{32-36}$. We hypothesize that intermittent use of acipimox, applied prior to exercise, might form an appropriate therapeutic strategy to allow intramuscular lipid and glycogen utilization, thereby further enhancing peripheral insulin sensitivity and, as such, improve postprandial glycemic control in patients with T2DM.

This combinational approach seems a promising alternative to enhance the clinical benefits of exercise therapy with equal effort of the patient on the long term, although one should be cautious about the potential side-effects of this combined therapy when used chronically. However, future work should be performed to explore the more prolonged benefits of combining low-intensity exercise training with adipose tissue lipolytic inhibition on glycemic control and clinical outcome in various diabetes subpopulations.

\section{Conclusion}

In conclusion, inhibition of adipose tissue lipolysis during exercise reduces circulatory plasma FFA concentration and lowers subsequent postprandial blood glucose and insulin excursions throughout the remainder of the day in male T2DM patients. These findings introduce the combined use of exercise with adipose tissue lipolytic inhibition as an effective interventional strategy to augment exercise-induced improvements in glycemic control in T2DM patients. 


\section{References}

1. de Vegt F, Dekker J, Ruhe H, Stehouwer C, Nijpels G, Bouter LM, Heine RJ (1999) Hyperglycaemia is associated with all-cause and cardiovascular mortality in the Hoorn population: the Hoorn Study. Diabetologia 42:926-931

2. Meigs J, Nathan D, D'Agostino RB Sr, Wilson P (2002) Fasting and postchallenge glycemia and cardiovascular disease risk: the Framingham Offspring Study. Diabetes Care 25:1845-1850

3. Bonora E, Corrao G, Bagnardi V, Ceriello A, Comaschi M, Montanari P, Meigs J (2006) Prevalence and correlates of post-prandial hyperglycemia in a large sample of patients with type 2 diabetes mellitus. Diabetologia 49:846-854

4. Woerle H, Neumann C, Zschau S, Tenner S, Irsigler A, Schirra J et al (2007) Impact of fasting and postprandial glycemia on overall glycemic control in type 2 diabetes: importance of postprandial glycemia to achieve target HbA1c levels. Diabetes Res Clin Pract 77:280-285

5. Weyer C, Bogardus C, Mott D, Pratley R (1999) The natural history of insulin secretory dysfunction and insulin resistance in the pathogenesis of type 2 diabetes mellitus. J Clin Invest 104:787-794

6. International Diabetes Federation.Guideline for management of postmeal glucose in diabetes. Available from http://www.idf.org/2011-guideline-managementpostmealglucose-diabetes. Accessed 12 February 2017

7. Colberg S, Sigal R, Fernhall B, Regensteiner J, Blissmer B, Rubin R et al (2010) Exercise and type 2 diabetes: the American College of Sports Medicine and the American Diabetes Association: joint position statement. Diabetes Care 33:e147-e167

8. American Diabetes Association. Standards of medical care in diabetes - 2017. Diabetes Care 2017; 40:S1-S8

9. van Dijk JW, van Loon L (2015) Exercise strategies to optimize glycemic control in type 2 diabetes: a continuing glucose monitoring perspective. Diabetes Spectr 28:2431

10. van Dijk JW, Manders R, Canfora E, Mechelen W, Hartgens F, Stehouwer C, van Loon L (2013) Exercise and 24-h glycemic control: equal effects for all type 2 diabetes patients? Med Sci Sports Exerc 45:628-635

11. Perseghin G, Price T, Petersen K, Roden M, Cline G, Gerow K et al (1996) Increased glucose transport-phosphorylation and muscle glycogen synthesis after exercise training in insulin-resistant subjects. N Engl J Med 335:1357-1362

12. Mikines K, Sonne B, Tronier B, Galbo H (1989) Effects of acute exercise and detraining on insulin action in trained men. J Appl Physiol 66:704-711

13. Praet $S$, van Loon L (2007) Optimizing the therapeutic benefits of exercise in type 2 diabetes. J Appl Physiol 103:1113-1120

14. Stinkens R, Goossens G, Jocken J, Blaak E (2015) Targeting fatty acid metabolism to improve glucose metabolism. Obes Rev 16:715-757

15. Goossens G, Moors C, Jocken J, van der Zijl N, Jans A et al (2016) Altered skeletal muscle fatty acid handling in subjects with impaired glucose tolerance as compared to impaired fasting glucose. Nutrients 8:164

16. Blaak E (2017) Characterisation of fatty acid metabolism in different insulin-resistant phenotypes by means of stable isotopes. Proc Nutr Soc 1-7

17. Corpeleijn E, Saris W, Blaak E (2009) Metabolic flexibility in the development of insulin resistance and type 2 diabetes: effects of lifestyle. Obes Rev 10:178-193

18. Samuel V, Shulman G (2016) The pathogenesis of insulin resistance: integrating signaling pathways and substrate flux. J Clin Invest 126:12-22 
19. Wojtaszewski J, Jorgensen S, Frosig C, MacDonald C, Birk JB, Richter EA (2003) Insulin signalling: effects of prior exercise. Acta Physiol Scand 178:321-328

20. van Loon $L$, Thomason-Hughes $M$, Constantin-Teodosiu $D$, Koopman $R$, Greenhaff PL, Grahame Hardie D et al (2005) Inhibition of adipose tissue lipolysis increases intramuscular lipid and glycogen use in vivo in humans. Am J Physiol Endocrinol Metab 289:E482-E493

21. van Loon L, Manders R, Koopman R, Kaastra B, Stegen JH, Gijsen AP et al (2005) Inhibition of adipose tissue lipolysis increases intramuscular lipid use in type 2 diabetic patients. Diabetologia 48:2097-2107

22. Fairbarn M, Blackie S, McElvaney N, Wiggs B, Paré P, Pardy R (1994) Prediction of heart rate and oxygen uptake during incremental and maximal exercise in healthy adults. Chest 105:1365-1369

23. American College of Sports Medicine (ACSM). ACSM's Guidelines for Exercise Testing and Prescription, $9^{\text {th }}$ Ed. Wolters Kluwer/Lippincott Williams \& Wilkins Health, 2014

24. Paap D, Takken T (2014) Reference values for cardiopulmonary exercise testing in healthy adults: a systematic review. Expert Rev Cardiovasc Ther 12:1439-1453

25. van Loon $L$ (2004) Use of intramuscular triacylglycerol as a substrate source during exercise in humans. J Appl Physiol 97:1170-1187

26. Tunaru S, Kero J, Schaub A, Wufka C, Blaukat A, Pfeffer K, Offermanns S (2003) PUMA-G and HM74 are receptors for nicotinic acid and mediate its anti-lipolytic effect. Nat Med 9:352-355

27. Watt M, Holmes A, Steinberg G, Mesa J, Kemp B, Febbraio M (2004) Reduced plasma FFA availability increases net triacylglycerol degradation, but not GPAT or HSL activity, in human skeletal muscle. Am J Physiol 287:E120-E127

28. Wojtaszewski J, Hansen B, Gade, Kiens B, Markuns J, Goodyear L, Richter E (2000) Insulin signaling and insulin sensitivity after exercise in human skeletal muscle. Diabetes 49:325-331

29. Di Pietro L, Dziura J, Yeckel C, Neufer P (2006) Exercise and improved insulin sensitivity in older women: evidence of the enduring benefits of higher intensity training. J Appl Physiol 100:142-149

30. Dube J, Allison K, Rousson V, Goodpaster B, Amati F (2012) Exercise dose and insulin sensitivity: relevance for diabetes prevention. Med Sci Sports Exerc 44:793-799

31. Cavalot F, Pagliarino A, Valle M, Di Martino L, Bonomo K, Massucco P et al (2011). Postprandial blood glucose predicts cardiovascular events and all-cause mortality in type 2 diabetes in a 14-year follow-up: lessons from the San Luigi Gonzaga Diabetes study. Diabetes Care 34:2237-2243

32. Wang W, Basinger A, Neese R, Christiansen M, Hellerstein M (2000) Effects of nicotinic acid on fatty acid kinetics, fuel selection, and pathways of glucose production in women. Am J Physiol Endocrinol Metab 279:E50-E59

33. Morigny P, Houssier M, Mouisel E, Langin D (2016) Adipocyte lipolysis and insulin resistance. Biochimie 125:259-266

34. Davoren P, Kelly W, Gries F, Hubinger A, Whately-Smith C, Alberti K (1998) Longterm effects of a sustained-release preparation of acipimox on dyslipidemia and glucose metabolism in non-insulin-dependent diabetes mellitus. Metabolism 47:250256

35. Vaag A, Beck-Nielsen H (1992) Effects of prolonged acipimox treatment on glucose and lipid metabolism and on in vivo insulin sensitivity in patients with non-insulin dependent diabetes mellitus. Acta Endocrinol Copenh 128:344-350 
36. Saloranta C, Groop L, Ekstrand A, Franssila-Kallunki A, Eriksson J, Taskinen M (1993) Different acute and chronic effects of acipimox treatment on glucose and lipid metabolism in patients with type 2 diabetes. Diabet Med 10:950-957 


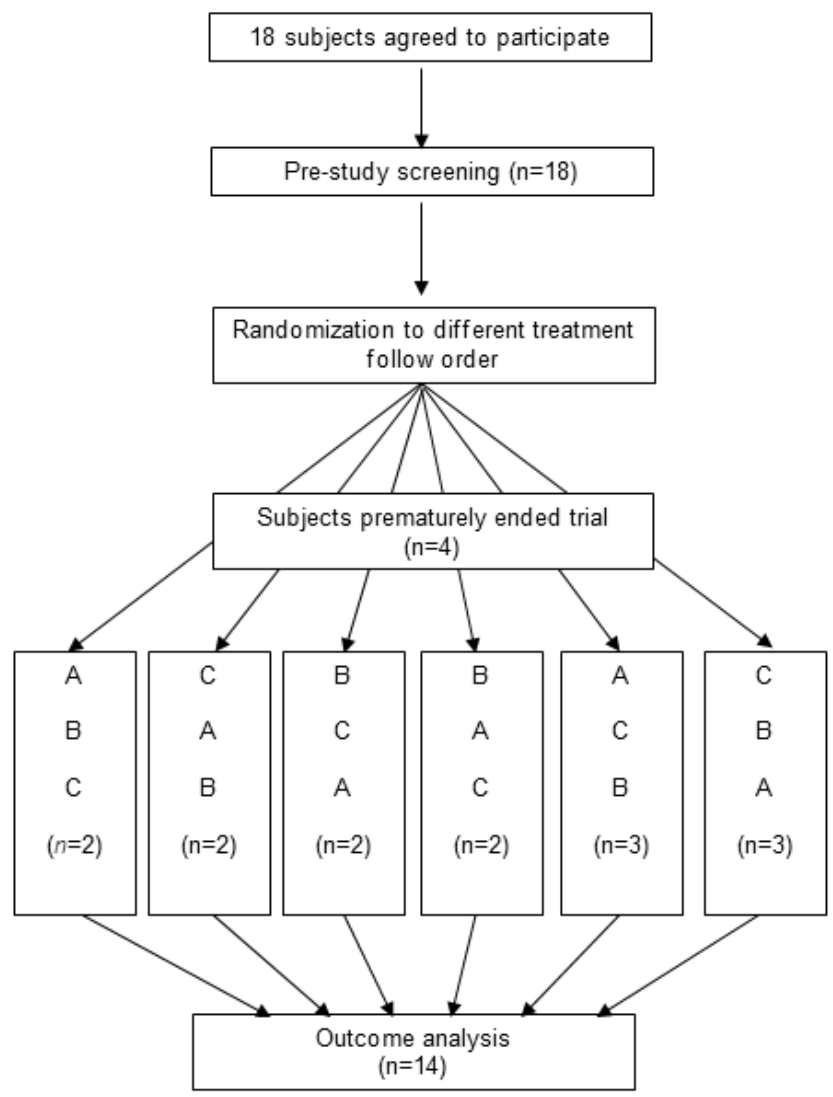

Supplemental Figure S1 - Flowchart and treatment randomization 
Chapter 7

General Discussion 
Obesity is a major ongoing health problem in our Western society ${ }^{1,2}$, a tendency that is mainly driven by changing lifestyle factors like increased energy intake 3,4 and reduced physical activity ${ }^{5}$. Excess adiposity and an impaired adipose tissue (AT) function does not only imply an increased risk for developing metabolic complications such as insulin resistance (IR), type 2 diabetes mellitus (T2DM) and cardiovascular diseases ${ }^{6}$, but also affects quality of life ${ }^{7}$ and reduces cardiometabolic health as it is often accompanied by the presence of hypertension, hyperlipidemias or metabolic syndrome ${ }^{1,8,9}$.

Adipose tissue dysfunction has been recognized as an imperative contributor to the obesity-related complications ${ }^{10,11}$, in which an impaired lipid buffering capacity ${ }^{12-14}$ and a reduced lipid turnover ${ }^{15-18}$ play an important role. Whereas excess AT is associated with the development of local and systemic low-grade inflammation ${ }^{19-22}$, altered storage, localization, handling and composition of lipids and lipid-intermediates might result in lipotoxicity, interfering with insulin signaling and promoting IR ${ }^{23-26}$ in skeletal muscle ${ }^{27-31}$ and liver ${ }^{24,32}$, thereby worsening cardiometabolic health. In particular, the lipid mobilization related impairments observed in obesity-related IR (as described earlier in this thesis) may favor increased fat stores and thus may be of primary importance for obesity-related metabolic complications. Consequently, improving AT lipid mobilization and other AT functional characteristics is a major concern in the prevention and treatment of obesity and obesity-related metabolic diseases. However, the importance of sub-phenotyping was recently recommended by the CardioMetabolic Health Alliance to provide tailored care ${ }^{33}$. Therefore, more insight into the dysfunctional AT characteristics of human obesity is required to more effectively direct prevention and treatment in metabolic diseases.

Therefore, in this thesis, by combining state-of-the-art in vivo clinical research with in vitro (ex vivo) mechanistic work, we investigated the endocrine regulation of AT lipid mobilization (one of the hallmarks of AT function), the impact of exercise intervention in human obesity and its association to obesityrelated IR in order to unravel its potential relevance in targeted obesity treatment. 


\section{Dysfunctional adipocyte lipolysis regulation in the obese state}

Regulating lipid availability and thus the release of lipids from its storage $\operatorname{depot}(\mathrm{s})$ and the subsequent delivery to non-adipose tissues, is of the utmost importance in the obese state to preserve cardiometabolic health. The insulin resistant skeletal muscle tissue often displays an impaired ability to increase fat oxidation upon increased supply ${ }^{28}$, and it therefore is important to strictly regulate AT lipolysis in order to prevent ectopic lipid accumulation. It is known from in vitro ${ }^{34}$, in situ ${ }^{35}$ and in vivo ${ }^{36-41}$ studies that catecholamine-induced lipolysis is blunted in the AT of obese subjects ${ }^{42}$, which was shown to be particularly present at the level of the abdominal subcutaneous adipose tissue (SCAT) ${ }^{35,43-46}$. Several physiological and molecular disturbances in the lipolytic pathway may underlie this catecholamine resistance. First, several receptor and post-receptor defects in the catecholamine signal transduction pathway are present in obese AT. A reduced number and function of $\beta$-adrenergic receptors 34,47 and an increased importance of the anti-lipolytic effects of $\alpha_{2}$-adrenergic receptors 48,49 have been reported in the insulin resistant SCAT. Next to catecholamines, of which adrenaline has been shown to be the major adrenergic regulator of adipocyte lipolysis ${ }^{50}$, other endocrine factors are involved in regulating lipid mobilization as will be discussed further in this chapter.

As described in Chapter 2, natriuretic peptides entered the lipolytic arena since the beginning of this millennium. Their role in metabolic control at the level of the AT, skeletal muscle and liver ${ }^{51-54}$, made this group of peptides attractive for human metabolic research. Moreover, their association with cardiovascular disease ${ }^{55-58}$ together with the presence of a systemic natriuretic deficiency in metabolic diseases (of which the cause remains incompletely understood) 59,60 emphasized natriuretic peptides to be a potential target in the treatment of obesity and other (cardio)metabolic diseases ${ }^{54}$. Although, additional research such as human in vivo studies or fundamental studies with human tissue material is warranted to unravel the molecular mechanisms being involved, thereby getting more insight into the causality chain between the etiology of metabolic disease and the natriuretic deficiency. 
With respect to the lipolytic properties of natriuretic peptides at the level of the AT 61,62 , we investigated in Chapter 3 whether adipocytes derived from abdominal subcutaneous and visceral (omental) AT of obese non-diabetic and obese diabetic individuals showed an impaired ANP-mediated lipolysis. We demonstrated an attenuated maximal responsiveness to ANP-mediated lipolytic stimulation in mature abdominal subcutaneous, but not visceral, adipocytes of obese men compared to lean individuals matched for age. This blunted maximal responsiveness was observed for both obese non-diabetic and obese diabetic men, indicating that this impairment can be attributed to the obese state per se. Additionally and in accordance with previous studies ${ }^{34-42}$, the lipolytic response to isoprenalin, a non-selective $\beta$-adrenergic agonist mimicking catecholamineinduced lipolytic effects, was blunted as well in obese abdominal subcutaneous adipocytes.

Our data on an impaired ANP-mediated lipolysis are in accordance with findings in obese women 63, where ANP-mediated lipolysis showed a lower responsiveness in the abdominal SCAT of obese versus non-obese women. Of special interest and for the first time reported in scientific literature, in Chapter 3 we showed that ANP-mediated lipolysis was reciprocally regulated in abdominal subcutaneous and visceral adipocytes in lean individuals, with abdominal subcutaneous adipocytes being more responsive to ANP stimulation as compared with visceral adipocytes. In contrast, this depot-specificity was absent in the obese non-diabetic and obese diabetic adipocytes. In line, previous data in middle-aged, morbidly obese women also showed no difference in ANPmediated lipolysis between abdominal subcutaneous and omental (i.e. visceral) in vitro differentiated adipocytes ${ }^{64}$. Thus, both ANP- and catecholaminemediated lipolysis are impaired in abdominal SCAT and not in the visceral adipose tissue (VAT) depot in human obesity and/or diabetes. This impairment may promote and/or maintain excess body fat accumulation in the overweight or obese state. 


\section{Obesity or an impaired insulin sensitivity, a culprit of altered ANP signaling?}

The presence of an impaired ANP-mediated lipolysis in abdominal SCAT in obese and obese diabetic subjects, led us to explore the underlying mechanism responsible for this anomaly. Natriuretic peptides exert their biological effects by binding the membrane-bound natriuretic peptide receptor A (NPRA), which stimulates the signaling cascade responsible for the functional/metabolic effects of ANP (and BNP) ${ }^{65}$. In contrast, besides intracellular degradation by neutral endopeptidase (NEP) 66,67 , the scavenging natriuretic peptide receptor type C (NPRC) is responsible for lysosomal degradation of its ligands such as ANP ${ }^{68,69}$.

Accordingly, in Chapter 3, the expression profiles of the functional NPRA and the scavenging NPRC were examined at the transcriptional (mRNA) as well as the translational (protein) level in order to (partly) unravel possible mechanisms for the impaired ANP-mediated lipolysis in human obesity. The scavenging NPRC is believed to affect ANP lipolytic effectiveness by binding ANP and subsequent intracellular degradation, precluding ANP from stimulating adipocytes ${ }^{68,69}$. The observed decrease in NPRA expression in SCAT of obese non-diabetic and obese diabetic individuals might be partly responsible for the observed attenuated ANP functionality. Besides, an increased NPRC mRNA expression was observed in both at the SCAT and VAT of obese non-diabetic and obese diabetic men. In addition, our NPRC mRNA levels were positively associated with whole-body IR (expressed as HOMA-IR) in both AT depots (with only VAT NPRC mRNA being associated independent of BMI), while the NPRA mRNA expression in SCAT was negatively associated with whole-body IR (independent of BMI) in our cohort. Our findings are in accordance with animal ${ }^{70}$ and human data ${ }^{71,72}$ showing a decreased natriuretic peptide receptor ratio (i.e. NPRA/NPRC) to be associated with obesity, glucose intolerance and IR.

Altered natriuretic peptide receptor expression might contribute to triglyceride accumulation, enlarged adipocytes, favoring IR and lipid abnormalities. Of interest, a reduced NPRC/NPRA gene expression ratio in VAT was associated to clinical metabolic parameters such as total serum cholesterol, LDL-cholesterol, non-HDL cholesterol, next to fasting insulinemia in overweight, insulin sensitive (mean HOMA-IR 1.6) men and women ${ }^{73}$. Nevertheless, the differential NPRC 
mRNA expression in the obese groups in the present study did not translate into a differential NPRC protein expression. This might possibly be explained by the rapid turnover of the NPRC receptor, whereby ligand-receptor complexes are rapidly internalized and degraded by lysosomes ${ }^{68}$.

However, according to classic receptor pharmacology principles ${ }^{74}$ differences in maximal responsiveness might reflect post-receptor signaling events. With respect to ANP-induced signal transduction, cyclic guanosine monophosphate (cGMP)-mediated signaling recently has been demonstrated to be repressed in visceral, but not subcutaneous, AT of obese humans, as shown by a decreased mRNA expression of protein kinase G (a downstream mediator of cGMPmediated signaling in adipocytes) ${ }^{75}$. In line, lipid droplet associated lipases (ATLG and HSL) ${ }^{76-80}$ and associated protein perilipin $1^{80,81}$ have been described to be decreased in SCAT of obese insulin resistant men, possibly contributing to the observed attenuated maximal ANP-mediated lipolysis in Chapter 3. Expression of phosphodiesterase 5 (PDE5), which hydrolyzes CGMP and thus impairs ANP-mediated signaling, was comparable between obese and non-obese individuals ${ }^{63}$. Moreover, the physiological relevance of PDE5 function in AT has been questioned since pharmacological inhibition in human subcutaneous ${ }^{82}$ and visceral ${ }^{83}$ adipocytes did not affect lipolytic rate. Despite the very limited evidence for a physiologically relevant role of AT cGMP signaling, we cannot draw firm conclusions about the cause of the impaired ANP-mediated lipolysis in human obesity from the present study and available literature. Nevertheless, targeting this pathway could be metabolically beneficial ${ }^{84-87}$.

Overall, in Chapter 3 both ANP- and catecholamine-mediated lipolysis were attenuated in SCAT of obese men with type 2 diabetes, that might be partially explained by NP receptor defects. Additionally, downstream signaling defects might have contributed to the blunted maximal ANP responsiveness as well. Therefore, improving maximal ANP responsiveness in adipose tissue, or lipolytic regulation in general, might be a potential future strategy to improve obesityassociated metabolic complications 


\section{Adipose tissue dysfunction and obesity-related insulin resistance - different disparities matter}

Dysfunctional adipose tissue is a known concept, associated with an increased cardiometabolic risk and characterised by an altered adipose tissue morphology, lipid metabolism and secretion of endocrine, paracrine and autocrine signals contributing to local and systemic low-grade inflammation and whole-body IR $10,88,89$. In Chapter 4, we studied the relevance of subcutaneous and visceral AT morphology, ex vivo lipolysis and immune cell populations in relation to wholebody IR in obese non-diabetic and type 2 diabetic (T2DM) men, compared with age-matched lean men.

Our data clearly indicated that visceral adipocyte hypertrophy is independently and positively associated with whole-body IR and thereby confirming previous studies ${ }^{90-92}$. This adipose tissue hypertrophy was accompanied by increased immune cell infiltration $\left(\mathrm{CD} 45^{+}\right.$leukocytes and pro-inflammatory M1 macrophages in particular), predominantly in the VAT of obese and obese diabetic individuals, which confirms previous studies showing an increased inflammation of this depot as compared to its subcutaneous counterpart 93,94 . Furthermore, multiple regression analyses with whole-body IR as a dependent variable revealed that VAT B-lymphocytes were independently associated with HOMA-IR. In SCAT, body fat mass was the major determinant of whole-body IR, whilst none of the AT functional characteristics were independently associated with whole-body IR.

Our data might partly reflect the commonly observed impaired buffering capacity of SCAT in the obese insulin resistant state ${ }^{95}$, which contributes to visceral adipocyte hypertrophy as a result of ectopic lipid deposition in this AT depot ${ }^{96,97}$. Notably, several human studies indicated the development of hyperinsulinemia, dyslipidemia and (peripheral) IR upon overfeeding without affecting the inflammatory phenotype of the SCAT ${ }^{98,99}$. In contrast to the role of AT immune cells in healthy AT expansion ${ }^{100-102}$, chronic AT inflammation results from the presence and/or activation of different immune cells in the hypertrophic AT ${ }^{103-106}$. The systemic inflammation in the obese insulin resistant state probably results from the pro-inflammatory phenotype of obese AT 107,108 and 
systemic inflammation may lead to impairments in insulin signaling and contributes to the development or maintenance of IR in insulin-sensitive metabolic tissues ${ }^{21,109}$ and T2DM and its complications ${ }^{110}$. Our novel results in human AT depots of metabolically compromised patients substantiate the role of adaptive immune cells (B lymphocytes) in the process of local AT inflammation in VAT, thereby (partly) confirming previous work in animals ${ }^{111-113 .}$

Taken together, the data in Chapter $\mathbf{4}$ showed that different cell populations in the immune landscape attribute to the known sub-clinical inflammatory state in obesity ${ }^{102}$ thereby broadening the classical view of AT macrophages being the major culprit of the inflammatory state in obesity ${ }^{20,114}$. We here confirm that the role of lymphocytes and macrophages in the obesity-related inflammatory carousel is undoubtable ${ }^{115}$. The interplay of immune and other AT cells orchestrating AT inflammation and function, as well as the mechanisms via which these cells impair insulin sensitivity require further investigation in preferentially longitudinal studies in metabolic disease.

\section{Modulation of adipose tissue lipid metabolism}

\section{Exercise}

Based on our observed attenuated ANP-mediated lipolysis in obese and obese diabetic SCAT (shown in Chapter 3), we examined the physiological relevance of exercise-induced ANP-mediated lipolysis in the abdominal SCAT of obese individuals in situ in Chapter 5. By locally and entirely blocking adrenergicmediated lipolytic stimulation during microdialysis at the level of the abdominal SCAT, using proper dosing of propranolol (a nonselective $\beta$-adrenergic receptor antagonist) ${ }^{116}$ and phentolamine (an $\alpha_{1,2}$-adrenergic receptor antagonist) ${ }^{117,118}$, we could investigate non-adrenergic-mediated lipolysis in the SCAT of lean insulin sensitive (IS), obese IS and obese insulin resistant (IR) individuals during rest, low-intensity endurance-type exercise and post-exercise recovery in the fasted state.

Our microdialysis data showed a reduced increase in exercise-mediated SCAT glycerol release in lean as compared to obese IS individuals. This discrepancy between phenotypes was, most likely, explained by a significant lower AT blood 
flow in the obese abdominal SCAT, thereby decreasing the shunting of mobilized lipids ${ }^{119-121}$ into the circulation. Although the difference in local AT blood flow (with or without pharmacological modification) hampers the lean vs obese comparison, this parameter certainly needs to be included in studies investigating local tissue effects.

Comparing obese IS and obese IR individuals (which displayed a comparable AT blood flow level), we observed a local reduction of SCAT lipolysis in the obese IS, not in obese IR, individuals following local $\alpha$-/ $\beta$-adrenergic blockade. These findings propose an interaction of AT adrenergic responsiveness with whole-body IR, which is in line with previous work $34,41,47$ and suggests a catecholamineresistant phenotype of the SCAT during exercise especially in the obese IR state. In addition, the presence of higher anti-lipolytic insulin ${ }^{29,122,123}$ and lactate ${ }^{124,125}$ concentrations in the obese IR might have contributed to the attenuated adrenergic-mediated SCAT lipolysis as compared to the obese IS group.

Our data postulate for a major role of non-adrenergic regulators of abdominal SCAT lipolysis during low-intensity endurance-type exercise. Several endocrine regulators with pro- or anti-lipolytic actions have been shown to regulate lipolysis only to a minor extent during this type of exercise ${ }^{126}$, so the main regulator (besides catecholamines and insulin) of SCAT lipolysis is suggested to be ANP 61, 127-129, especially in the overweight/obese state ${ }^{130,131}$. Our data showed that more than half of the lipolytic response remained present under local $\alpha-/ \beta$-adrenergic blockade in the lean and obese (IS and IR) state. Therefore these results suggest a major role of non-adrenergic- (probably ANP-) mediated AT lipolysis during exercise in abdominal SCAT, which is even sustained in the obese IR state as the anti-lipolytic action of insulin does not affect ANP-mediated lipolytic signaling 132-134. Notably, the lack of an ANP receptor antagonist limits the strength of the proposed contribution of ANP in the physiological control of adipose tissue lipid mobilization. Still, the relevance of ANP is, in our opinion, established as it is currently the most important factor governing AT lipolysis during acute exercise bouts. Understanding its modulatory properties in the lipolytic arena therefore seems imperative in order to orchestrate the synergy between the different lipolytic regulatory pathways to further ameliorate AT metabolism in metabolic compromised conditions. 
Exercise intervention has been known for its important role in causing fat mass loss and ameliorating metabolic profiles (including insulin sensitivity) in obese individuals ${ }^{135}$. In line, as described in Chapter 5, a 12-week exercise intervention (including endurance- and resistance-type exercise training) improved physical fitness and body composition in obese individuals. Of interest, exercise-induced systemic plasma responses (in particular plasma glycerol, FFA and lactate) improved in both obese groups and whole-body IR improved in the obese IR individuals. Together these data indicate the clinical efficacy of our exercise training program, which was applied to the current guidelines for obesity treatment ${ }^{136}$.

Previous studies in overweight and obese individuals have shown that exercise training has beneficial effects on adipocyte lipolysis and its regulation, including improvements in insulin ${ }^{137}$ and adrenergic sensitivity ${ }^{137-143}$ at the level of the SCAT of obese individuals. Moreover, long-term endurance exercise training demonstrated a recovery of the reduced subcutaneous ANP responsiveness and ANP-mediated lipolysis in overweight individuals and obese women in situ 144 and in obese women with polycystic ovary syndrome in vitro and in situ ${ }^{142}$, indicating the susceptibility of ANP-mediated AT lipolysis. In Chapter 5, the effect(s) of a combined endurance and resistance exercise intervention on the endocrine regulation of abdominal SCAT lipolysis were determined in obese IR individuals. However, exercise-induced abdominal SCAT lipolysis was not changed as a result of exercise intervention in the obese IR individuals. Since we only observed a weak trend towards a small increment of adrenergic blockade efficacy in the SCAT of obese IR individuals following exercise intervention, these modest improvements may result from the low number of individuals included or the intensity of the applied exercise program, which can modulate the clinical efficacy in metabolic disease 145,146. In addition, combining calorie restriction together with (high intensity) exercise training may be preferred ${ }^{147}$.

Overall, the results described in Chapter 5 postulate for a major role ANPmediated lipolysis during exercise in both lean and obese individuals and an apparent reduced adrenergically-mediated lipolysis in obese IR versus obese IS individuals. However, regardless of improvements in metabolic profile and body composition, adipocyte lipolytic impairments remain present in the obese state. 
Therefore, optimized therapies are warranted to achieve enhancements in AT lipolysis regulation, especially in metabolically compromised individuals.

\section{Pharmacological targeting of adipose tissue lipid metabolism during exercise}

The role of lipotoxicity and, in particular, lipid spill-over has been known to impair insulin signaling and to promote IR at the level of different insulinsensitive organs including skeletal muscle and liver, making AT lipid metabolism an important target to improve glucose homeostasis ${ }^{32}$. In line, preclinical studies ${ }^{148-154}$ have suggested that partial inhibition of lipolysis, using nicotinic acid or its analog acipimox, may be a promising therapeutic strategy in obese metabolically compromised people. Therefore, the clinical study described in Chapter 6 substantiated the role of fatty acids in the regulation of glycemic control in obese men with T2DM.

In Chapter 6, our double-blind, placebo-controlled cross-over trial in obese T2DM individuals showed that inhibiting in vivo AT lipolysis by means of acipimox (a well-known analog of nicotinic acid specifically inhibiting adipose HSL ${ }^{155}$ ) during a single bout of endurance-type exercise in the fasted state resulted in alleviated consecutive postprandial glycemia and insulinemia, as opposed to exercise alone or a sedentary control experiment. However, the beneficial glucoregulatory effects of endurance-type exercise in the fasted state under acipimox intake in Chapter 6 were only present in the subsequent two postprandial phases but were no longer present the morning after, during an oral glucose tolerance test.

In clinical treatment T2DM it is of major importance to enhance the efficacy of exercise bouts in T2DM patients as many T2DM patients suffer from an impaired exercise tolerance ${ }^{156}$. In addition, to achieve long-term glycemic control targets 157 , it is important to optimize/maximize the glucoregulatory properties of each individual acute exercise bout ${ }^{158,159}$, for which our applied combined approach (including acipimox intake during endurance-type exercise) described in

Chapter 6 could be a potential option. In this regard, sedentary T2DM patients might benefit the most from this combined therapy when starting the exercise intervention (since their exercise capacity is mostly limited at this point). Upon 
enhanced exercise tolerance, exercise training can become the main focus, thereby gradually reducing acipimox dosage.

Of interest, one should be aware of the possible deteriorating effects of chronic treatment with nicotinic acid, which affect glucose metabolism and insulin sensitivity in the opposite way ${ }^{149,150}$ due to a rebound effect on plasma FFA concentrations ${ }^{151,160,161}$. Therefore, the use of acipimox is preferred because of its more favorable effects on insulin sensitivity ${ }^{152,153,162}$, glycated hemoglobin ${ }^{163}$ and glucose metabolism ${ }^{162,164-167 .}$

The clinical suitability of this therapy in combination with exercise has definitely some promising potential implications, in which the intermittent use of acipimox (only during exercise) may be a therapeutic option in metabolic disease to circumvent the potential rebound effect of chronic acipimox treatment and to alleviate the often observed insulin resistant state. To fully realize the potential of acipimox-mediated lowering of plasma FFA on improving glucose control, net FFA fluxes rather than solely circulating FFA should be achieved ${ }^{148,154,168}$. In this respect, intermittent exposure profiles in humans might improve the current clinical use and metabolic outcomes of acipimox treatment ${ }^{154,161,169}$.

Of interest, as the feeding status might influence the efficacy of metabolic outcome ${ }^{169}$, the practical use of this combined therapy might, in our opinion, be limited to the fasted (as opposed to the post-prandial) state. Consequently, these questions need to be addressed in future human intervention studies. 


\section{Conclusion and future perspectives}

This thesis focuses on the endocrine regulation of AT lipid mobilization and the impact of exercise intervention in obese individuals, with a specific emphasis on the atrial natriuretic peptide (ANP), a heart-derived protein involved in human substrate and energy metabolism.

We found a pronounced attenuation of ANP-mediated lipolysis in the abdominal SCAT of obese individuals due to alterations at the receptor level, although this resistant-like phenotype was not observed in visceral adipocytes. In our exercise intervention study investigating the regulation of SCAT lipolysis in obese IS and IR individuals, the physiological relevance of this regulatory pathway was confirmed in the obese state. Of interest, controlling AT function in the obese state is crucial to maintain whole-body insulin sensitivity, the latter which was shown to be modulated by an exercise training program in obese individuals or its combination with partial pharmacological modulation of AT lipolysis in T2DM patients.

The main outcomes of this thesis provide evidence for recommending the importance of a proper regulation of AT lipid metabolism in order to maintain a 'healthy' AT function. We showed the presence of an impaired adipocyte lipolysis regulation in the SCAT of obese insulin resistant and obese T2DM patients, which could not be reversed by moderate-intense exercise intervention. Optimizing the clinical efficacy of exercise in the treatment of obesity and T2DM is of primary importance to improve obesity-related metabolic complications and therefore new strategies need to be explored in clinical practice. However, several questions and issues should be addressed in future research:

1. Our main finding in Chapter $\mathbf{3}$ was the presence of an impaired ANPresponsiveness at the level of the SCAT in obesity-related IR and type 2 diabetes. Hence, the importance of ANP-mediated regulation during exercise of adipocyte lipolysis in the obese state was proposed in Chapter 5. However, despite several human studies suggested a metabolic role of natriuretic peptides including ANP, the lack of suitable ANP receptor antagonists limits the conclusive nature of these suggestions in humans. Therefore, the need for this type of molecules in human in vivo experiments is imperative to further unravel the 
complexity of human adipocyte lipolysis regulation. In addition, tissues biopsies of adipose tissue and skeletal muscle should be taken to elaborate on underlying mechanisms.

2. In Chapter 5, we suggest the importance of ANP to sustain a proper lipolytic response during exercise in the obese state, whereby its role in the obese insulin resistant state might be of relatively increased importance due to the presence of catecholamine resistance. However, we did not observe any difference in systemic ANP concentrations between different obese phenotypes, nor between obese and lean individuals, which contrasts previous findings indicating the presence of a systemic natriuretic deficiency in human metabolic diseases, of which the cause remains to be determined. Therefore, more fundamental approaches are necessary in order to clarify the molecular mechanisms contributing to this natriuretic deficiency (e.g. attenuated cardiac secretion mechanisms or increased systemic/peripheral clearance of these peptides). Such experiments could include human intervention or prospective studies, mechanistic experiments with cultures of cardiomyocytes (principal producers of natriuretic peptides) or in vitro work on the tissue level to further exploring the difficulties with respect to tissue-specific receptors and signaling pathways.

3. In our studies, we included male obese and obese diabetic individuals. In literature, evidence also highlights gender differences in adipose tissue function, with a plausible role of sex hormones in differential regional white adipose tissue function. Still, the mechanisms controlling the regulation of gender-specific fat distribution remain poorly understood. Of interest, the repercussions of adipose tissue depot- and gender-specific effects on adipocyte function, as well as the relationship to cardiometabolic health and the efficacy of diet/exercise intervention are not fully elucidated and warrant further analysis.

4. Getting more insights into the molecular changes in the different lipolytic cascades would apply not only to obesity prevention but also to the elucidation of a methodology for advances in exercise efficacy. Especially with respect to ANP and its associated lipolytic signaling pathway, a lot of aspects are to be explored in future research. Together with mechanistic cell culture studies, these 
experiments would gain more fundamental data for future optimization of exercise based therapies in the prevention of chronic metabolic disease.

5. As AT function, and in particular AT lipid metabolism, might be modified by both pharmacological and non-pharmacological intervention, the question remains which are the true molecular mechanisms going on during these intervention, especially with respect to metabolic organs like the AT or the skeletal muscle tissue. These mechanisms might (partly) clarify the observed integral lipolytic impairments present in the obese state, even following exercise intervention. Furthermore, the effects on AT function are mostly multifactorial (e.g. ameliorating AT lipid metabolism, AT inflammation, amongst others) leaving a lot of opportunities to investigate in metabolic research. Elucidating these multiple sub-characteristics of AT function and the effects of distinctive therapeutical approaches hereon may serve as base for improving obesity and metabolic disease treatment in clinical practice.

6. The important observation of our exercise intervention study, described in Chapter 5, that a 12-week training intervention did not translate into pronounced improvement of the lipolytic response effects at the SCAT in our obese populations with different insulin sensitivity despite improvement in body composition and metabolic profile, also raised further questions. First, is a three month period of this type of intensity, as prescribed by international guidelines ${ }^{136}$, sufficient to induce changes in AT lipolysis regulation in these phenotypes? Second, are interventional approaches equally effective in improving metabolic health or AT function in different metabolic profiles (e.g. fat distribution, insulin sensitivity, cardiac burden, amongst others), or do we need tailored therapies for different metabolic profile (personalized medicine)? The use of combinational therapy (e.g. as applied in Chapter 6), including lifestyle changes, diet intervention and/or pharmacological methods could be of great interest to investigate in future metabolic research. 


\section{References}

1. World Health Organization. http://www.who/int/mediacentre/factssheets/fs311/en/, updated June 2016

2. Ng M, Fleming T, Robinson M, Thomson B, Graetz N, Margono C et al (2014) Global, regional and national prevalence of overweight and obesity in children and adults during 1980-2013: a systematic analysis for the Global Burden of Disease Study 2013. Lancet 384:766-781

3. Bertéus Forslund H, Torgerson JS, Sjöström L, Lindroos A (2005) Snacking frequency in relation to energy intake and food choices in obese men and women compared to a reference population. Int J Obes 29:711-719

4. Van der Klaauw A, Faroogi I (2015) The hunger genes: pathways to obesity. Cell 161:119-132

5. Blundell J, Gibbons C, Caudwell P, Finlayson G, Hopkins M (2015) Appetite control and energy balance: impact of exercise. Obes Rev 16 Suppl1:67-76

6. Emerging Risk Factors C, Wormser D, Kaptoge S, Di Angelantonio E, Wood A, Pennells $L$ et al (2011) Separate and combined associations of body-mass index and abdominal adiposity with cardiovascular disease: collaborative analysis of 58 prospective studies. Lancet 377:1085-1095

7. Forhan M, Gill S (2013) Obesity, functional mobility and quality of life. Best practice \& research Clinical endocrinology \& metabolism 27:129-137

8. Mokdad A, Ford E, Bowman B, Dietz W, Vinicor F, Bales V, Marks J (2003) Prevalence of obesity, diabetes, and obesity-related health risk factors, 2001. JAMA 289:76-79

9. Zimmet P, Magliano D, Matsuzawa Y, Alberti G, Shaw J (2005) The metabolic syndrome: a global public health problem and a new definition. J Atheroscler Thromb 12:295-300

10. Goossens G (2008) The role of adipose tissue dysfunction in the pathogenesis of obesity-related insulin resistance. Physiol Behav 94:206-218

11. Rosen E, Spiegelman B (2014) What we talk about when we talk about fat. Cell 156:20-44

12. Krotkiewski M, Björntorp $P$, Sjöström L, Smith U (1983) Impact of obesity on metabolism in men and women. Importance of regional adipose tissue distribution. J Clin Invest 72:1150-1162

13. Potts J, Coppack S, Fisher R, Humphreys S, Gibbons G, Frayn K (1995) Impaired postprandial clearance of triacylglycerol-rich lipoproteins in adipose tissue in obese subjects. Am J Physiol 268:E588-E594

14. Lundgren M, Svensson M, Lindmark S, Renström F, Ruge T, Eriksson J (2007) Fat cell enlargement is an independent marker of insulin resistance and hyperleptinaemia. Diabetologia 50:625-633

15. Jocken J, Blaak E (2008) Catecholamine-induced lipolysis in adipose tissue and skeletal muscle in obesity. Physiol Behav 94:219-230

16. Nellemann B, Gormsen L, Sorensen L, Christiansen J, Nielsen S (2012) Impaired insulin-mediated antilipolysis and lactate release in adipose tissue of upper-body obese women. Obesity 20:57-64

17. Arner P, Bernard S, Salehpour M, Possnert G, Liebl J, Steier P et al (2011) Dynamics of human adipose lipid turnover in health and metabolic disease. Nature 478:110113

18. Rydén M, Andersson D, Bernard S, Spalding K, Arner P (2013) Adipocyte triglyceride turnover and lipolysis in lean and overweight subjects. J Lipid Res 54:2909-2913 
19. Hotamisligil G, Shargill N, Spiegelman B (1993) Adipose expression of tumor necrosis factor-alpha: direct role in obesity-linked insulin resistance. Science 259:87-91

20. Weisberg S, McCann D, Desai M, Rosenbaum M, Leibel R, Ferrante A (2003) Obesity is associated with macrophage accumulation in adipose tissue. $\mathrm{J}$ Clin Invest 112:1796-1808

21. Makki K, Froguel P, Wolowczuk I (2013) Adipose tissue in obesity-related inflammation and insulin resistance: cells, cytokines, and chemokines. ISRN Inflamm 2013:139239

22. Bai Y, Sun Q (2015) Macrophage recruitment in obese adipose tissue. Obes Rev 16:127-136

23. Machann J, Häring $H$, Schick F, Stumvoll M (2004) Intramyocellular lipids and insulin resistance. Diabetes Obes Metab 6:239-248

24. Perry R, Zhang X, Zhang D, Kumashiro N, Camporez J, Cline G et al (2014) Leptin reverses diabetes by suppression of thehypothalamic-pituitary-adrenal axis. Nat Med 20:759-763

25. Glass C, Olefsky J (2012) Inflammation and lipid signaling in the etiology of insulin resistance. Cell Metab 15:635-645

26. Blaak E (2017) Characterisation of fatty acids metabolism in different insulinresistant phenotypes by means of stable isotopes. Proc Nutr Soc 19:1-7

27. Blaak E, van Aggel-Leijssen D, Wagenmakers A, Saris W, van Baak M (2000) Impaired oxidation of plasma-derived fatty acids in type 2 diabetic subjects during moderate-intensity exercise. Diabetes 49:2102-2107

28. Blaak E, Hul G, Verdich C, Stich V, Martinez A, Petersen M et al (2006) Fat oxidation before and after a high fat load in the obese insulin-resistant state. J Clin Endocrinol Metab 91:1462-1469

29. Jocken J, Goossens G, Boon H, Mason R, Essers Y, Havekes B et al (2013) Insulinmediated suppression of lipolysis in adipose tissue and skeletal muscle of obese type 2 diabetic men and men with normal glucose tolerance. Diabetologia 56:2255-2265

30. Samuel V, Shulman G (2016) The pathogenesis of insulin resistance: integrating signaling pathways and substrate flux. J Clin Invest 126:12-22

31. Ritter O, Jelenik T, Roden M (2015) Lipid-mediated muscle insulin resistance: different fat, different pathways. J Mol Med 93:831-843

32. Stinkens R, Goossens G, Jocken J, Blaak E (2015) Targeting fatty acid metabolism to improve glucose metabolism. Obes Rev 16:715-57

33. Sperling L, Mechanick J, Neeland I, Herrick C, Després J, Ndumele C et al (2015) The CardioMetabolic Health Alliance: Working Toward a New Care Model for the Metabolic Syndrome. J Am Coll Cardiol 66:1050-1067

34. Reynisdottir S, Wahrenberg H, Carlstrom K, Rossner S, Arner P (1994) Catecholamine resistance in fat cells of women with upper-body obesity due to decreased expression of beta 2-adrenoceptors. Diabetologia 37:428-435

35. Horowitz J, Klein S (2000) Whole body and abdominal lipolytic sensitivity to epinephrine is suppressed in upper body obese women. Am J Physiol Endocrinol Metab 278:E1144-E1152

36. Connacher A, Bennet W, Jung R, Bier D, Smith C, Scrimgeour C, Rennie M (1991) Effect of adrenaline infusion on fatty acid and glucose turnover in lean and obese human subjects in the post-absorptive and fed states. Clin Sci 81:635-644

37. Blaak E, Van Baak M, Kemerink G, Pakbiers M, Heidendal G, Saris W (1994) Beta adrenergic stimulation of energy expenditure and forearm skeletal muscle metabolism in lean and obese men. Am J Physiol 267: E306-E315 
38. Blaak E, Van Baak M, Kemerink G, Pakbiers M, Heidendal G, Saris W (1994) Betaadrenergic stimulation of skeletal muscle metabolism in relation to weight reduction in obese men. Am J Physiol 267: E316-E322

39. Webber J, Taylor J, Greathead H, Dawson J, Buttery P, Macdonald I (1994) A comparison of the thermogenic, metabolic and haemodynamic responses to infused adrenaline in lean and obese subjects. Int J Obes Relat Metab Disord 18:717-724

40. Jensen $M$ (1997) Lipolysis: contribution from regional fat. Annu Rev Nutr 17:127-139

41. Jocken J, Goossens G, van Hees A, Frayn K, van Baak M, Stegen J et al (2008) Effect of beta-adrenergic stimulation on whole-body and abdominal subcutaneous adipose tissue lipolysis in lean and obese men. Diabetologia 51:320-327

42. Arner P (1999) Catecholamine-induced lipolysis in obesity. Int J Obes Relat Metab Disord 23 Suppl 1:10-13

43. Arner $P(1998)$ Not all fat is alike. Lancet 351:1301-1302

44. Enoksson S, Talbot M, Rife F, Tamborlane W, Sherwin R, Caprio S (2000) Impaired in vivo stimulation of lipolysis in adipose tissue by selective beta2-adrenergic agonist in obese adolescent girls. Diabetes 49:2149-2153

45. Wajchenberg B, Giannella-Neto D, da Silva M, Santos R (2002) Depot-specific hormonal characteristics of subcutaneous and visceral adipose tissue and their relation to the metabolic syndrome. Horm Metab Res 34:616-621

46. Lafontan M, Berlan M (2003) Do regional differences in adipocyte biology provide new pathophysiological insights. Trends Pharmacol Sci 24:276-283

47. Lafontan M, Berlan M (1993) Fat cell adrenergic receptors and the control of white and brown fat cell function. J Lipid Res 34:1057-1091

48. Stich V, De Glisezinski I, Crampes F, Hejnova J, Cottet-Emard J et al (2000) Activation of alpha(2)-adrenergic receptors impairs exercise-induced lipolysis in SCAT of obese subjects. Am J Physiol Regul Integr Comp Physiol 279:R499-R504

49. Richterova B, Stich V, Moro C, Polak J, Klimcakova E, Majercik M et al (2004) Effect of endurance training on adrenergic control of lipolysis in adipose tissue of obese women. J Clin Endocrinol Metab 89:1325-1331

50. De Glisezinski I, Larrouy D, Bajzova M, Koppo K, Polak J, Berlan M et al (2009) Adrenaline but not noradrenaline is a determinant of exercise-induced lipid mobilization in human subcutaneous adipose tissue. J Physiol 587:3393-3404

51. Schlueter N, de Sterke A, Willmes D, Spranger J, Jordan J, Birkenfeld A (2014) Metabolic actions of natriuretic peptides and therapeutic potential in the metabolic syndrome. Pharmacol Ther 144:12-27

52. Zois N, Bartels E, Hunter I, Kousholt B, Olsen L, Goetze J (2014) Natriuretic peptides in cardiometabolic regulation and disease. Nat Rev Cardiol 11:403-412

53. Coué M, Moro C (2016) Natriuretic peptide control of energy balance and glucose homeostasis. Biochimie 124:84-91

54. Moro C (2016) Targeting cardiac natriuretic peptides in the therapy of diabetes and obesity. Expert Opin Ther Targets 20:1445-1452

55. Volpe M (2014) Natriuretic peptides and cardio-renal disease. In J Cardiol 176:630639

56. Sharma A, Ahmed V, Garg A, Aggarwal C (2015) Clinical Applications of Natriuretic Peptides in Assessment of Valvular Heart Disease. Dis Markers 2015:807861

57. Ramos H, Birkenfeld A, de Bold A (2015) INTERACTING DISCIPLINES: Cardiac natriuretic peptides and obesity: perspectives from an endocrinologist and a cardiologist. Endocr Connect 4:R25-R36

58. Omland T, White $\mathrm{H}$ (2017) State of the Art: Blood Biomarkers for Risk Stratification in Patients with Stable Ischemic Heart Disease. Clin Chem 63:165-176 
59. Olsen M, Hansen T, Christensen M, Gustafsson F, Rasmussen S, Wachtell K et al (2005) N-terminal pro brain natriuretic peptide is inversely related to metabolic cardiovascular risk factors and the metabolic syndrome. Hypertension 46:660-666

60. Wang T, Larson M, Levy D, Benjamin E, Leip E, Wilson P et al (2004) Impact of obesity on plasma natriuretic peptide levels. Circulation 109:594-600

61. Sengenes C, Berlan M, De Glisezinski I, Lafontan M, Galitzky J (2000) Natriuretic peptides: a new lipolytic pathway in human adipocytes. FASEB J 14:1345-1351

62. Sengenes C, Zakaroff-Girard A, Moulin A, Berlan M, Bouloumié A, Lafontan M, Galitzky J (2002) Natriuretic peptide-dependent lipolysis in fat cells is a primate specificity. Am J Physiol Regul Integr Comp Physiol 283:R257-R265

63. Rydén $M$, Backdahl J, Petrus $P$, Thorell A, Gao H, Coue M et al (2016) Impaired atrial natriuretic peptide-mediated lipolysis in obesity. Int J Obes 40:714-20

64. Dicker A, Aström G, Wahlén K, Hoffstedt J, Näslund E, Wirén M et al (2009) Primary differences in lipolysis between human omental and subcutaneous adipose tissue observed using in vitro differentiated adipocytes. Horm Metab Res 41:350-355

65. Khun M (2016) Molecular physiology of membrane guanylyl cyclase receptors. Physiol Rev 96:751-804

66. Kenny A, Bourne A, Ingram J (1993) Hydrolysis of human and pig brain natriuretic peptides, urodilatin, C-type natriuretic peptide and some C-receptor ligands by endopeptidase-24.11. Biochem J 1:83-88

67. Schling $P$, Löffer $G$ (2002) Cross talk between adipose tissue cells: impact on pathophysiology. New Physiol Sci 17:99-104

68. Pandey K (1992) Kinetic analysis of internalization, recycling and redistribution of atrial natriuretic factor-receptor complex in cultured vascular smooth-muscle cells. Ligand-dependent receptor down-regulation. Biochem J 288:55-61

69. Matsukawa N, Grzesik W, Takahashi N, Pandey K, Pang S, Yamauchi M, et al (1999) The natriuretic peptide clearance receptor locally modulates the physiological effects of the natriuretic peptide system. PNAS 96:7403-7408

70. Nakatsuji H, Maeda N, Hibuse T, Hiuge A, Hirata A, Kuroda Y et al (2010) Reciprocal regulation of natriuretic peptide receptors by insulin in adipose cells. Biochem Biophys Res Comm 392:100-105

71. Pivovarova O, Gögebakan Ö, Klöting N, Sparwasser A, Weickert M, Haddad I et al (2012) Insulin up-regulates natriuretic peptide clearance receptor expression in the subcutaneous fat depot in obese subjects: a missing link between CVD risk and obesity. J Clin Endocrinol Metab 94:E731-E739

72. Kovacova Z, Tharp W, Liu D, Wei W, Xie H, Collins S et al (2016) Adipose tissue natriuretic peptide receptor expression is related to insulin sensitivity in obesity and diabetes. Obesity 24:820-8

73. Bordicchia M, Ceresiani M, Pavani M, Minardi D, Polito M, Wabitsch M et al (2016) Insulin/glucose induces natriuretic peptide clearance receptor in human adipocytes: a metabolic link with the cardiac natriuretic pathway. Am J Physiol Regul Integr Comp Physiol 311:R104-R114

74. Kenakin T (2004) Principles: receptor theory in pharmacology. Trends Pharmacol Sci 25:186-92

75. Sanyal A, Naumann J, Hoffmann L, Chabowska-Kita A, Ehrlund A, Schlitzer A et al (2017) Interplay between Obesity-Induced Inflammation and cGMP Signaling in White Adipose Tissue. Cell Rep 18:225-236

76. Large V, Reynisdottir S, Langin D, Fredby K, Klannemark M, Holm C, Arner P (1999) Decreased expression and function of adipocyte hormone-sensitive lipase in subcutaneous fat cells of obese subjects. J Lipid Res 40:2059-2066 
77. Rydén M, Jocken J, van Harmelen V, Dicker A, Hoffstedt J, Wirén M et al (2007) Comparative studies of the role of hormone-sensitive lipase and adipose triglyceride lipase in human fat cell lipolysis. Am J Physiol Endocrinol Metab 292:E1847-E18455

78. Jocken J, Langin D, Smit E, Saris W, Valle C, Hul G, Holm C et al (2007) Adipose triglyceride lipase and hormone-sensitive lipase protein expression is decreased in the obese insulin-resistant state. J Clin Endocrinol Metab 92:2292-2299

79. Langin D, Dicker A, Tavernier G, Hoffstedt J, Mairal A, Rydén M et al (2005) Adipocyte lipases and defect of lipolysis in human obesity. Diabetes 54:3190-3197

80. Ray H, Pinteur C, Frering V, Beylot M, Large V (2009) Depot-specific differences in perilipin and hormone-sensitive lipase expression in lean and obese. Lipids Health Dis 8:58

81. Moreno-Navarette J, Ortega F, Serrano M, Rodriguez-Hermosa J, Ricart W, Mingrone G, Fernandez-Real J (2014) CIDEC/FSP27 and PLIN1 gene expression run in parallel to mitochondrial genes in human adipose tissue, both increasing after weight loss. Int J Obes 38:865-872

82. Moro C, Klimcakova E, Lafontan M, Berlan M, Galitzky J (2007) Phosphodiesterase-5A and neutral endopeptidase activities in human adipocytes do not control atrial natriuretic peptide-mediated lipolysis. Br J Pharmacol 152:1102-1110

83. Aversa A, Caprio M, Antelmi A, Armani A, Brama M, Greco E et al (2011) Exposure to phosphodiesterase type 5 inhibitors stimulates aromatase expression in human adipocytes in vitro. J Sex Med 8:696-704

84. Ayala J, Bracy D, Julien B, Rottman J, Fueger P, Wasserman D (2007) Chronic treatment with sildenafil improves energy balance and insulin action in high fat-fed conscious mice. Diabetes 56:1025-1033

85. Handa P, Tateya S, Rizzo N, Cheng A, Morgan-Stevenson V, Han C (2011) Reduced vascular nitric oxide-cGMP signaling contributes to adipose tissue inflammation during high-fat feeding. Arterioscler Thromb Vasc Biol 31:2827-2835

86. Hoffmann L, Etzrodt J, Willkomm L, Sanyal A, Scheja L, Fischer A (2015) Stimulation of soluble guanylyl cyclase protects against obesity by recruiting brown adipose tissue. Nat Commun 6:7235

87. Pfeifer A, Hoffmann L (2015) Brown, beige, and white: the new color code of fat and its pharmacological implications. Annu Rev Pharmacol Toxicol 55:207-227

88. Després J (2012) Abdominal obesity and cardiovascular disease: is inflammation the missing link. Can J Cardiol 28:642-652

89. Van Greevenbroek M, Schalkwijk C, Stehouwer C (2016) Dysfunctional adipose tissue and low-grade inflammation in the management of the metabolic syndrome: current practices and future advances. F1000Res 5(F1000 Faculty Rev):2515

90. Weyer C, Foley J, Bogardus C, Tataranni P, Pratley R (2000) Enlarged subcutaneous abdominal adipocyte size, but not obesity itself, predicts type II diabetes independent of insulin resistance. Diabetologia 43:1498-1506

91. Lönn M, Mehlig K, Bengtsson C, Lissner L (2010) Adipocyte size predicts incidence of type 2 diabetes in women. FASEB J 24:326-331

92. Acosta J, Douagi I, Andersson D, Bäckdahl J, Rydén M, Arner P, Laurencikiene J (2016) Increased fat cell size: a major phenotype of subcutaneous white adipose tissue in non-obese individuals with type 2 diabetes. Diabetologia 59:560-570

93. Cancello R, Henegar C, Viguerie N, Taleb S, Poitou C, Rouault C et al (2005) Reduction of macrophage infiltration and chemoattractant gene expression changes in white adipose tissue of morbidly obese subjects after surgery-induced weight loss. Diabetes 54:22777-22786 
94. Harman-Boehm I, Blüher M, Redel H, Sion-Vardy N, Ovadia S, Avinoach E et al (2007) Macrophage infiltration into omental versus subcutaneous fat across different populations: effect of regional adiposity and the comorbidities of obesity. J Clin Endocrinol Metab 92:2240-2247

95. Kim S, Lun M, Wang M, Senyo S, Guillermier C, Patwari P, Steinhauser M (2014) Loss of White Adipose Hyperplastic Potential Is Associated with Enhanced Susceptibility to Insulin Resistance. Cell Metab 20:1049-1058

96. Jensen M, Sarr M, Dumesic D, Southorn P, Levine J (2003) Regional uptake of meal fatty acids in humans. Am J Physiol Endocrinol Metab 285:E1282-E1288

97. Vortruba S, Mattison R, Dumesic D, Koutsari C, Jensen M (2007) Meal fatty acid uptake in visceral fat in women. Diabetes 56:2589-2597

98. Tam CS, Viardot A, Clément K, Tordjman J, Tonks K, Greenfield JR et al (2010) Short-term overfeeding may induce peripheral insulin resistance without altering subcutaneous adipose tissue macrophages in humans. Diabetes 59:2164-2170

99. Johannsen DL, Tchoukalova Y, Tam CS, Covington JD, Xie W, Schwarz JM et al (2014) Effect of 8 weeks of overfeeding on ectopic fat deposition and insulin sensitivity: testing the adipose tissue expandability hypothesis. Diabetes Care 37:2789-2797

100. Ferrante A (2013) The Immune Cells in Adipose Tissue. Diabetes Obes Metab 15 Suppl 3:34-38

101. Brestoff J, Artis D (2015) Immune regulation of metabolic homeostasis in health and disease. Cell 161:146-160

102. Blüher M (2016) Adipose tissue inflammation: a cause or consequence of obesityrelated insulin resistance Clin Sci 130:1603-1614

103. Kanda H, Tateya S, Tamori Y, Kotani K, Hiasa K, Kitazawa R et al (2006) MCP-1 contributes to macrophage infiltration into adipose tissue, insulin resistance, and hepatic steatosis in obesity. J Clin Invest 116:1494-1505

104. Masoodi M, Kuda O, Rossmeisl M, Flachs P, Kopecky J (2015) Lipid signaling in adipose tissue: Connecting inflammation \& metabolism. Biochim Biophys Acta 1851:503-518

105. Osborn O and Olefsky J (2012) The cellular and signaling networks linking the immune system and metabolism in disease. Nat Med 18:363-374

106. Mathis D (2013) Immunological goings-on in visceral adipose tissue. Cell Metab 17:851-859

107. Wynn T, Chawla A, Pollard J (2013) Macrophage biology in development, homeostasis and disease. Nature 496:445-455

108. Mraz M, Haluzik M (2014) The role of adipose tissue immune cells in obesity and lowgrade inflammation. J Endocrinol 222:R113-R127

109. Goossens G, Blaak E, Theunissen R, Duijvestijn A, Clément K, Tervaert J, Thewissen $M$ (2012) Expression of NLRP3 inflammasome and $T$ cell population markers in adipose tissue are associated with insulin resistance and impaired glucose metabolism in humans. Mol Immunol 50:142-149

110. Meshkani R, Vakili S (2016) Tissue resident macrophages: Key players in the pathogenesis of type 2 diabetes and its complications. Clin Chim Acta 462:77-89

111. Kintscher U, Hartge M, Hess K, Foryst-Ludwig A, Clemenz M, Wabitsch M et al (2008) T-lymphocyte infiltration in visceral adipose tissue: a primary event in adipose tissue inflammation and the development of obesity-mediated insulin resistance. Arterioscler Thromb Vasc Biol 28:1304-1310 
112. Winer D, Winer S, Shen L, Wadia P, Yantha J, Paltser G et al (2011) B lymphocytes promote insulin resistance through modulation of $\mathrm{T}$ lymphocytes and production of pathogenic IgG antibody. Nat Med 17:610-617

113. DeFuria J, Belkina A, Jagannathan-Bogdan M, Snyder-Cappione J, Carr J, Nersesova $Y$ et al (2013) B cells promote inflammation in obesity and type 2 diabetes through regulation of $\mathrm{T}$ cell function and an inflammatory cytokine profile. Proc Natl Acad Sci USA 110:5133-5138

114. Xu H, Barnes G, Yang Q, Tan G, Yang D, Chou C et al (2003) Chronic inflammation in fat plays a crucial role in the development of obesity-related insulin resistance. J Clin Invest 112:1821-1830

115. Cinkajzlova A, Mraz M, Haluzik M (2017) Lymphocytes and macrophages in adipose tissue in obesity: markers or makers of subclinical inflammation. Protoplasma [Epub ahead of print] doi: 10.1007/s00709-017-1082-3

116. Moro C, Crampes F, Sengenes C, De Glisezinski I, Galitzky J, Thalamas C et al (2004) Atrial natriuretic peptide contributes to physiological control of lipid mobilization in humans. FASEB J 18:908-910

117. Stich V, De Glisezinski I, Crampes F, Hejnova J, Cottet-Emard J, Galitzky J et al (2000) Activation of alpha(2)-adrenergic receptors impairs exercise-induced lipolysis in SCAT of obese subjects. Am J Physiol Regul Integr Comp Physiol 279:R499-R504

118. Polak J, Moro C, Bessiere D, Hejnova J, Marques M, Bajzova M et al. Acute exposure to long-chain fatty acids impairs \{alpha\}2-adrenergic receptor-mediated antilipolysis in human adipose tissue. J Lipid Res 48:2236-2246

119. Bulow J (1981) Human adipose tissue blood flow during prolonged exercise, III. Effect of beta-adrenergic blockade, nicotinic acid and glucose infusion. Scand J Clin Lab Invest 41:415-424

120. Goossens G, Jocken J, Blaak E, Schiffers P, Saris W, van Baak M (2007) Endocrine role of the renin-angiotensin system in human adipose tissue and muscle: effect of beta-adrenergic stimulation. Hypertension 49:542-547

121. Summers L, Samra J, Humphreys S, Morris R, Frayn K (1996) Subcutaneous abdominal adipose tissue blood flow: variation within and between subjects and relationship to obesity. Clin Sci 91:679-683

122. Lafontan M, Langin D (2009) Lipolysis and lipid mobilization in human adipose tissue. Prog Lipid Res 48:275-297.

123. Hershkop K, Besor O, Santoro N, Pierpont B, Caprio S, Weiss R (2016) Adipose Insulin Resistance in Obese Adolescents Across the Spectrum of Glucose Tolerance. J Clin Endocrinol Metab 101:2423-2431

124. Ahmed K, Tunaru S, Tang C, Müller M, Gille A, Sassmann A et al (2010) An autocrine lactate loop mediates insulin-dependent inhibition of lipolysis through GPR81. Cell Metab 11:311-319

125. Liu C, Wu J, Zhu J, Kuei C, Yu J, Shelton J et al (2009) Lactate inhibits lipolysis in fat cells through activation of an orphan G-protein-coupled receptor, GPR81. J Biol Chem 284:2811-2822

126. Hansen D, Meeusen R, Mullens A, Dendale P (2012) Effect of acute endurance and resistance exercise on endocrine hormones directly related to lipolysis and skeletal muscle protein synthesis in adult individuals with obesity. Sports Med 42:415-431

127. Follenius M, Brandenberger G (1988) Increase in atrial natriuretic peptide in response to physical exercise. Eur J Appl Physiol Occup Physiol 57:159-162

128. Berlin I, Deray G, Lechat P, Maistre G, Landault C, Chermat V et al (1993) Tertatolol potentiates exercise-induced atrial natriuretic peptide release by increasing atrial diameter in healthy subjects. Cardiology 83 Suppl 1:16-24 
129. Niessner A, Ziegler S, Slany J, Billensteiner E, Woloszczuk W, Geyer G (2003) Increases in plasma levels of atrial and brain natriuretic peptides after running a marathon: are their effects partly counterbalanced by adrenocortical steroids. Eur J Endocrinol 149:555-559

130. Moro C, Polak J, Hejnova J, Klimcakova E, Crampes F, Stich V et al (2006) Atrial natriuretic peptide stimulates lipid mobilization during repeated bouts of endurance exercise. Am J Physiol Endocrinol Metab 290:E864-E869

131. Moro C, Pillard F, de Glisezinski I, Klimcakova E, Crampes F, Thalamas C et al (2008) Exercise-induced lipid mobilization in subcutaneous adipose tissue is mainly related to natriuretic peptides in overweight men. Am J Physiol Endocrinol Metab 295:E505E513

132. Sengenes C, Bouloumie A, Hauner H, Berlan M, Busse R, Lafontan M et al (2003) Involvement of a cGMP-dependent pathway in the natriuretic peptide-mediated hormone-sensitive lipase phosphorylation in human adipocytes. J Biol Chem 278:48617-48626

133. Lönnroth P, Smith U (1986) The antilipolytic effect of insulin in human adipocytes requires activation of the phosphodiesterase. Biochem Biophys Res Commun 141:1157-1161

134. Makino $H$, Suzuki T, Kajinuma H, Yamazaki M, Ito H, Yoshida S (1992) The role of insulin-sensitive phosphodiesterase in insulin action. Adv Second Messenger Phosphoprotein Res 25:185-199

135. Chin S, Kahathuduwa C, Binks M (2016) Physical activity and obesity: what we know and what we need to know. Obes Rev 17:1226-1244

136. Yumuk V, Tsigos C, Fried M, Schindler K, Busetto L, Micic D et al (2015) European Guidelines for Obesity Management in Adults. Obes Facts 8:402-424

137. Polak J, Moro C, Klimcakova E, Hejnova J, Majercik M, Viguerie N et al (2005) Dynamic strength training improves insulin sensitivity and functional balance between adrenergic alpha $2 \mathrm{~A}$ and beta pathways in subcutaneous adipose tissue of obese subjects. Diabetologia 48:2631-2640

138. Stich V, de Glisezinski I, Galitzky J, Hejnova J, Crampes F, Rivière D, Berlan M (1999) Endurance training increases the beta-adrenergic lipolytic response in subcutaneous adipose tissue in obese subjects. Int J Obes Relat Metab Disord 23:374-381

139. Richterova B, Stich V, Moro C, Polak J, Klimcakova E, Majercik M, et al (2004) Effect of endurance training on adrenergic control of lipolysis in adipose tissue of obese women. J Clin Endocrinol Metab 89:1325-1331

140. De Glisezinski I, Crampes F, Harant I, Berlan M, Hejnova J, Langin D et al (1998) Endurance training changes in lipolytic responsiveness of obese adipose tissue. Am J Physiol 275:E951-E956

141. De Glisezinski I, Moro C, Pillard F, Marion-Latard F, Harant I, Meste M et al (2003) Aerobic training improves exercise-induced lipolysis in SCAT and lipid utilization in overweight men. Am J Physiol Endocrinol Metab 285:E984-E990

142. Moro C, Pasarica M, Elkind-Hirsch K, Redman L (2009) Aerobic exercise training improves atrial natriuretic peptide and catecholamine-mediated lipolysis in obese women with polycystic ovary syndrome. J Clin Endocrinol Metab 94:2579-2586

143. Crampes F, Marion-Latard F, Zakaroff-Girard A, De Glisezinski I, Harant I, Thalamas $C$ et al (2003) Effects of a longitudinal training program on responses to exercise in overweight men. Obes Res 11:247-256 
144. Moro C, Pillard F, De Glisezinski I, Harant I, Rivière D, Stich V et al (2005) Training Enhances ANP Lipid-Mobilizing Action in Adipose Tissue of Overweight Men. Medicine \& Science in Sports \& Exercise 37:1126-1132

145. Jelleyman C, Yates T, O'Donovan G, Gray L, King J, Khunti K, Davies M (2015) The effects of high-intensity interval training on glucose regulation and insulin resistance: a meta-analysis. Obes Rev 16:942-961

146. Cassidy S, Thoma C, Houghton D, Trenell M (2017) High-intensity interval training: a review of its impact on glucose control and cardiometabolic health. Diabetologia 60:7-23

147. Weiss E, Albert S, Reeds D, Kress K, Ezekiel U, McDaniel J et al (2015) Calorie restriction and matched weight loss from exercise: independent and additive effects on glucoregulation and the incretin system in overweight women and men. Diabetes Care 38:1253-1262

148. Girousse A, Tavernier G, Valle C, Moro C, Mejhert N, Dinel A et al (2013) Partial inhibition of adipose tissue lipolysis improves glucose metabolism and insulin sensitivity without alteration of fat mass. PLoS Biol 11:e1001485

149. Balasse E and Neef M (1973) Influence of nicotinic acid on the rates of turnover and oxidation of plasma glucose in man. Metabolism 22:1193-1204

150. Miettinen T, Taskinen M, Pelkonen R, Nikkilä E (1969) Glucose tolerance and plasma insulin in man during acute and chronic administration of nicotinic acid. Acta Med Scand 186:247-253

151. Wang W, Basinger A, Neese R, Christiansen M, Hellerstein M (2000) Effects of nicotinic acid on fatty acid kinetics, fuel selection, and pathways of glucose production in women. Am J Physiol Endocrinol Metab 279:E50-E59

152. Santomauro A, Boden G, Silva M, Rocha D, Santos R, Ursich M et al (1999) Overnight lowering of free fatty acids with Acipimox improves insulin resistance and glucose tolerance in obese diabetic and nondiabetic subjects. Diabetes 48:1836-1841

153. Vaag A, Skött P, Damsbo P, Gall M, Richter E, Beck-Nielsen H (1991) Effect of the antilipolytic nicotinic acid analogue acipimox on whole-body and skeletal muscle glucose metabolism in patients with non-insulin-dependent diabetes mellitus. J Clin Invest 88:1282-1290

154. Kroon T, Kjelstedt A, Thalén P, Gabrielsson J, Oakes N (2015) Dosing profile profoundly influences nicotinic acid's ability to improve metabolic control in rats. J Lipid Res 56:1679-1690

155. Tunaru S, Kero J, Schaub A, Wufka C, Blaukat A, Pfeffer K, Offermanns S (2003) PUMA-G and HM74 are receptors for nicotinic acid and mediate its anti-lipolytic effect. Nat Med 9:352-355

156. Colberg S, Sigal R, Fernhall B, Regensteiner J, Blissmer B, Rubin R et al (2010) Exercise and type 2 diabetes: the American College of Sports Medicine and the American Diabetes Association: joint position statement. Diabetes Care 33:e147e167

157. Colberg S, Sigal R, Yardley J, Riddell M, Dunstan D, Dempsey P et al (2016) Physical Activity/Exercise and Diabetes: A Position Statement of the American Diabetes Association. Diabetes Care 39:2065-2079

158. Praet $S$, van Loon $L$ (2007) Optimizing the therapeutic benefits of exercise in type 2 diabetes. J Appl Physiol 103:1113-1120

159. van Dijk JW, van Loon L (2015) Exercise strategies to optimize glycemic control in type 2 diabetes: a continuing glucose monitoring perspective. Diabetes Spectr 28:24-31 
160. Garg A and Grundy S (1990) Nicotinic acid as therapy for dyslipidemia in non-insulindependent diabetes mellitus. JAMA 264:723-726

161. Andersson R, Kroon T, Almquist J, Jirstrand M, Oakes N, Evans N et al (2017) Modeling of free fatty acid dynamics: insulin and nicotinic acid resistance under acute and chronic treatments. J Pharmacokinet Pharmacodyn [Epub ahead of print] doi: 10.1007/s10928-017-9512-6

162. Liang $H$, Tantiwong $P$, Sriwijitkamol A, Shanmugasundaram K, Mohan S, Espinoza S et al (2013) Effect of a sustained reduction in plasma free fatty acid concentration on insulin signalling and inflammation in skeletal muscle from human subjects. J Physiol 591:2897-2909

163. Davoren P, Kelly W, Gries F, Hubinger A, Whately-Smith C, Alberti K (1998) Longterm effects of a sustained-release preparation of acipimox on dyslipidemia and glucose metabolism in non-insulin-dependent diabetes mellitus. Metabolism 47:250256

164. Bajaj M, Suraamornkul S, Kashyap S, Cusi K, Mandarino L, DeFronzo R (2004) Sustained reduction in plasma free fatty acid concentration improves insulin action without altering plasma adipocytokine levels in subjects with strong family history of type 2 diabetes. J Clin Endocrinol Metab 89:4649-4655

165. Daniele G, Eldor R, Merovci A, Clarke G, Xiong J, Tripathy D et al (2014) Chronic reduction of plasma free fatty acid improves mitochondrial function and whole-body insulin sensitivity in obese and type 2 diabetic individuals. Diabetes 63:2812-2820

166. Vaag A and Beck-Nielsen H (1992) Effects of prolonged Acipimox treatment on glucose and lipid metabolism and on in vivo insulin sensitivity in patients with noninsulin dependent diabetes mellitus. Acta Endocrinol (Copenh) 127:344-350

167. Saloranta C, Groop L, Ekstrand A, Franssila-Kallunki A, Eriksson J, Taskinen M (1993) Different acute and chronic effects of acipimox treatment on glucose and lipid metabolism in patients with type 2 diabetes. Diabet Med 10:950-957

168. Arner P, Rydén M (2015) Fatty Acids, obesity and insulin resistance. Obes Facts 8:147-155

169. Kroon T, Baccega T, Olsén A, Gabrielsson J, Oakes N (2017) Nicotinic acid timed to feeding reverses tissue lipid accumulation and improves glucose control in obese Zucker rats. J Lipid Res 58:31-41 

ADDENDUM

Valorisation 
The present thesis describes the potential of targeting the endocrine regulation of adipocyte lipolysis in human obesity treatment and prevention. An appropriate adipose tissue lipid metabolism is imperative to maintain normal adipose tissue function in human metabolically compromised conditions like obesity or type 2 diabetes mellitus. The valorization potential of the current results will be described in terms of social and economic relevance and implications for specific target groups and future innovative development.

\section{Social and economic relevance of the obesity epidemic}

Currently, our society is facing an alarming increase of overweight and obesity prevalence, which even extends to developing countries. According to latest statistics of the World Health Organisation (2014), Europe had the second highest proportion of individuals with overweight or obesity, preceded by the Americas. In Belgium and the Netherlands, $67 \%$ and $49 \%$ of the adult population deals with overweight and even $22 \%$ and $14 \%$ are diagnosed with obesity, respectively. The future perspectives on the development of obesity are disconcerting since it is predicted that the number of individuals with obesity will increase even more in the next two decades. This is a perplexing trend as individuals with obesity are more prone to develop metabolic diseases, cardiovascular diseases, mental disorders and certain types of cancer. Moreover, obesity (together with obesity-related complications) will further hamper efforts for healthcare cost management and thus not only affects public health but also has major socio-economic consequences. Therefore, new or optimized strategies for treatment and prevention are warranted to counteract this global obesity epidemic. Obtaining more insights into the adipose tissue physiology, and in particular adipose tissue lipid metabolism, is crucial with respect to treatment optimization and therefore was the main focus of the current thesis.

\section{Development of obesity and obesity-related health complications}

Obesity results from a chronic positive energy balance in which energy intake exceeds energy expenditure. Consequently, the energy surplus is stored within the adipose tissue, which gradually expands and may result in adipose tissue dysfunction. The main pillars of restoring the energy balance are reducing energy intake in relation to energy expenditure and increasing energy 
expenditure. However, despite diet intervention being one of the most studied interventions in obesity treatment, many people often cannot maintain their initial weight loss due to a high relapse (weight regain) rate in this type of interventions. Contrarily, stimulating energy expenditure could be a suitable alternative to restore or even create a negative energy balance, in which increased physical activity or exercise is a common used strategy. Despite the efficacy of exercise, with respect to induce weight loss and promote healthier metabolic outcomes, many people often have difficulties maintaining regular physical activity regimes in their everyday life, ultimately limiting weight loss and in particular limiting weight maintenance after weight loss. Furthermore, the observed biomechanical anomalies often present in the obese state frequently hamper the ability to perform physical exercise. Developing alternative (combinational) strategies to improve clinical efficacy (i.e. maximizing weight loss and restoring metabolic complications) in the obese state are therefore imperative. With respect to weight loss, the stimulation of adipocyte lipolysis, a physiological process that is tightly regulated in an endocrine manner, is essential to reduce adipose tissue mass in the context of a negative energy balance.

Long-term regulation and control of body weight (body composition) relies upon a large regulatory network integrating different metabolic organs, in which the white adipose tissue is the major place for long-term energy storage in the form of triacylglycerol in adipocytes. In obesity, the chronic positive energy balance enlarges the adipocytes which may lead to an impaired lipid buffering capacity of the adipose tissue, which together with intrinsic lipolytic impairments may be identified as putative key factors in the pathophysiology of obesity and obesityrelated metabolic and cardiometabolic complications. Gaining better insight into the role of these anomalies in obesity pathophysiology as well as lipolytic endocrine regulation is warranted in human metabolically compromised conditions. Therefore, in this thesis we explored whether natriuretic peptides, and in particular atrial natriuretic peptide (ANP), are substantially involved in the physiological regulation of adipocyte lipolysis in the obese insulin resistant state. Moreover, another goal was to explore the effect(s) of combined exercise intervention on this endocrine regulation system. As confirmed in the present thesis, there is now significant evidence that ANP in involved in the regulation of 
adipocyte lipolysis, especially in the abdominal subcutaneous adipose tissue. However, in the obese insulin resistant or obese diabetic state, adipose tissue lipolysis displays a catecholamine- and ANP-resistant phenotype, which was not improved upon exercise intervention in the obese insulin resistant state. Therefore, finding appropriate strategies to alleviate the observed lipolytic impairments in the obese insulin resistant state are of major importance for body weight control and to improve adipose tissue function and metabolic profile in humans with obesity and thus more research in humans is required.

\section{Target groups}

Given the development of obesity associated metabolic disease affecting millions of people in our current societies, finding strategies to prevent and treat overweight, obesity and obesity-related health risks are crucial to improve global health and to counteract exploding healthcare costs. Indeed, in the obese state, even modest weight loss might lead to substantial beneficial health effects. Therefore, optimizing clinical efficacy of intervention programs, in terms of adipose tissue mass reduction and metabolic health, are of primary interest. In this respect, developing strategies, which are able to restore known lipolytic impairments present in the obese insulin resistant adipose tissue could be of major interest. To develop targeted exercise interventions or better pharmacological therapies should include fundamental as well as clinical work in human individuals with obesity and/or obesity-related metabolic complications.

In order to tackle obesity development, and in particular the development of its associated metabolic diseases like type 2 diabetes mellitus and cardiovascular disease, health care professionals, health institutions (including hospital settings, rehabilitation and sports centers), governments and general society need to be informed about these targeted exercise or combinational treatment (e.g. lifestyle intervention including exercise, diet and/or pharmaceutical treatments) strategies. In science, the aim is to make results available for the scientific community and health professionals through publication in international peer-reviewed journals and presentation on (inter)national scientific meetings. However, translation of scientific knowledge to society is essential. Beyond academia and scientific journals and meetings, our research 
has been published in newspapers which enabled us to reach a broader audience to take notice of our findings. In this thesis, by performing in vitro work and clinical intervention studies with human subjects, we demonstrated a pronounced attenuation of ANP-mediated lipolysis in the abdominal subcutaneous adipocytes of obese individuals. The physiological relevance of this pathway in adipose tissue lipolysis was confirmed in the obese state as well, although exercise intervention could not restore the observed anomaly. Yet, exercise training in individuals with obesity or its combination with partial pharmacological inhibition of adipose tissue lipolysis in patients with type 2 diabetes was shown to modulate whole-body insulin sensitivity. Thus, the results presented in this thesis are of clinical relevance for a large number of people and therefore may be used by health care professionals to optimize lifestyle programs for individuals with overweight or obesity. In order to stimulate the translation of these findings to society and obtain awareness and support from the general public or policy makers, communicating these observations outside science is of considerable importance. Of interest, next to lifestyle and behavioral intervention to target obesity or its metabolic complications, pharmacological (co)interventions may be an alternative to alleviate adipose tissue function in the obese state. Targeting adipose tissue lipid metabolism or adipocyte function via pharmaceutical agents requires a good understanding and collaboration between universities and the pharmaceutical industry.

\section{Activities and products}

The research described in this thesis has been conducted at the department of Human Biology (Maastricht University), the Rehabilitation Research Center (Hasselt University) in collaboration with Heart Center Hasselt and Abdominal Surgery of Jessa Hospital and Hospital East-Limburg (Belgium). This unique collaboration between Universities and hospitals is a good example of how basic science can be performed in a hospital setting, combining knowledge of scientists and medical specialists across boarders. The collaboration between basic scientists and professionals involved in obesity management is essential given that the current applied physical activity modalities in obesity rehabilitation, which are applied very frequent at both aforementioned hospital departments, might not be appropriate to restore adipose tissue function in 
these patients. In order to increase the knowledge on white adipose tissue function and thereby improve prevention and/or treatment of human metabolically compromised conditions, strong national and international collaboration between different disciplines is of utmost importance.

The results described in this thesis are published or will be published in original research articles in scientific journals in the field of obesity and diabetes. Accordingly, these articles can be found online (accessible to those interested). The present results help to understand white adipose tissue physiology in humans and can be used for future studies on exploring optimized strategies to combat the obesity epidemic. The key role of adipose tissue function in metabolic health is currently subject of debate in the field of obesity research. The main outcomes of this thesis provide evidence for recommending the importance of a proper regulation of adipose tissue lipid metabolism in order to maintain a 'healthy' adipose tissue function. Moreover, this thesis showed that exercise training was not able to improve adipose tissue lipolysis despite improvements in metabolic disturbances, indicating the need for substantial weight loss to further restore adipose tissue function. However, the repercussions of adipose tissue depot- and sex-specific effects on adipocyte function, as well as the relationship to cardiometabolic health and the efficacy of diet/exercise intervention are important aspects, which require further research. This might also imply that stratification of individuals with obesity based on metabolic health characteristics is necessary to optimize and create tailored prevention and treatment strategies.

\section{Innovation}

By combining state-of-the-art in vivo clinical research and laboratory analyses, we were able to investigate for the first time the contribution of ANP in the regulation of adipocyte lipolysis in individuals with obesity. Moreover, the use of exercise intervention was shown to be insufficient to restore the observed impaired adipocyte lipolysis regulation in the obese insulin resistant state, leaving some opportunities for future research (as described in Chapter 6 and 7 of this thesis). 
The current findings indicate a possible important role of non-adrenergically mediated lipolysis, most likely (partly) mediated by ANP, especially during physical activity or exercise. Together with the observations of the presence of a putative natriuretic deficiency in metabolic disease (as described in Chapter 2), this might indicate that the natriuretic peptide system or some of their components (e.g. their receptors or downstream mediators at the level of the adipose tissue) are candidate drug targets with therapeutic potential in obesity and type 2 diabetes, population in which poor adherence to lifestyle intervention is frequently observed. However, the therapeutic potential should be explored guardedly in future research, with special attention to cardiovascular complications. Of interest, efficient and selective (i.e. adipose tissue specific) ANP receptor agonists and antagonists are warranted to firmly delineate the physiological relative contribution of the different lipolytic agents in adipocyte lipolysis. 


\section{APPENDICES}

Summary 
Over the last decades, the prevalence of obesity has dramatically increased, reaching epidemic proportions and which is expected to rise even more the coming decennia. Obesity is an important risk factor for developing cardiometabolic complications, having a substantial impact on general health status and thus health care. Due to an imbalance between energy storage and energy expenditure in the obese state, energy is stored in the adipose tissue. Upon excessive weight gain, adipose tissue dysfunction develops, which is associated with ectopic lipid accumulation and the development of metabolic complications like insulin resistance or type 2 diabetes mellitus (Chapter $\mathbf{1}$ ). Therefore, an appropriate adipose tissue lipid metabolism is imperative to maintain healthy adipose tissue function in human obesity. Consequently, improving adipose tissue lipid mobilization (and other adipose tissue functional characteristics) is a major concern in the prevention and treatment of obesity and obesity-related metabolic diseases. As it is important to strictly regulate adipose tissue lipid mobilization (also called lipolysis) in order to prevent ectopic lipid accumulation, the studies described in this thesis investigated the endocrine regulation of adipose tissue lipid mobilization. Moreover, the impact of exercise intervention (and its combination with pharmaceutical inhibition of adipose tissue lipolysis) on adipose tissue lipolysis in human obesity and its association with obesity-related insulin resistance were investigated in order to unravel its potential in obesity treatment and prevention.

Accumulating evidence indicates that natriuretic peptides are involved in metabolic control at the level of insulin-sensitive tissue including adipose tissue, skeletal muscle and liver, making these peptides attractive for human metabolic research. Moreover, their role in the regulation of adipose tissue lipid mobilization (Chapter 2) led us to explore whether natriuretic peptides, and in particular atrial natriuretic peptide (ANP), are substantially involved in the physiological regulation of adipose tissue lipolysis in the obese insulin resistant state.

In Chapter 3, we demonstrated an attenuated maximal responsiveness to ANPmediated lipolytic stimulation in isolated mature abdominal subcutaneous, but not visceral, adipocytes of obese men with and without type 2 diabetes compared to age-matched lean individuals. This blunted maximal 
responsiveness might be explained by natriuretic peptide receptor defects (and post-receptor signaling defects) present in the obese insulin resistant state. Interestingly, the depot-dependent differential regulation of ANP-mediated lipolysis in abdominal subcutaneous and visceral adipocytes of lean individuals was absent in adipocytes of individuals with obesity, thereby promoting and/or maintaining excess body fat accumulation in the obese state.

Next to adipose tissue lipid metabolism, other dysfunctional adipose tissue phenotypic characteristics (e.g. adipocyte hypertrophy, adipose tissue inflammation) may contribute to an increased cardiometabolic risk in the obese state. In Chapter 4, we demonstrated the importance of visceral adipocyte hypertrophy and visceral B-lymphocytes as independent contributors to wholebody insulin resistance (as assessed by the surrogate marker HOMA-IR). With respect to the subcutaneous adipose tissue depot, fat mass rather than adipose tissue function variables was related to whole-body insulin resistance. The subclinical adipose tissue inflammatory state in the human obesity was corroborated by increased expression profiles of total lymphocytes and pro-inflammatory M1macrophages in the visceral adipose tissue (as compared to lean individuals), thereby indicating the presence of an orchestrated interplay between different cell types within the obese adipose tissue.

As described in Chapter 3, mechanistic work using in vitro (ex vivo) techniques indicated an ANP-resistant like phenotype at the level of the subcutaneous adipose tissue of individuals with obesity. The physiological relevance of ANPmediated lipolysis during exercise was examined by using state-of-the-art microdialysis experiments at the level of the subcutaneous adipose tissue of individuals with obesity. The results of Chapter $\mathbf{5}$ indicate a major role of nonadrenergic regulators of adipocyte lipolysis during low-intensity endurance-type exercise in human obesity, presumably ANP-driven. However, regardless of improvements in metabolic profile and body composition, adipocyte lipolytic impairments remain present in the obese state following an exercise training program, suggesting the need for optimized therapeutic strategies to prevent or treatment obesity. Therefore, in Chapter 6, we demonstrated the clinical suitability and efficacy of a combined approach in which pharmacological lowering of plasma free fatty acids by means of the nicotinic acid analog 
acipimox, used as a tool to inhibit adipose tissue lipolysis, together with low- to moderate-intensity endurance-type exercise in the fasted state was able to alleviate consecutive postprandial glycemia and insulinemia in obese individuals with type 2 diabetes.

In conclusion, and based on all data gathered in this thesis, a proper regulation of adipose tissue lipid metabolism is of utmost importance in order to maintain a 'healthy' adipose tissue function. The role of ANP in regulating adipocyte lipolysis was shown to be of physiological relevance, especially in metabolically compromised conditions like the obese insulin resistant or type 2 diabetic state, where we observed a blunted catecholamine- and ANP-mediated lipolytic response at the level of the subcutaneous adipose tissue. The observed lipolytic impairments could not be reversed by exercise intervention, postulating the need for novel add-on therapies to further optimize the efficacy of (combined) prevention and treatment strategies implemented in the clinical care of individuals with obesity. 


\section{APPENDICES}

Samenvatting 
De prevalentie van zwaarlijvigheid of obesitas is de afgelopen decennia enorm toegenomen, waarbij reeds epidemische proporties worden aangenomen. De huidige voorspellingen geven aan dat deze cijfers de komende decennia zelfs nog meer zullen stijgen. Obesitas is een sterke risicofactor voor de ontwikkeling van cardiovasculaire en metabole complicaties, wat een aanzienlijke impact heeft op de algemene gezondheid en leidt tot een stijgende druk op de gezondheidszorg. Bij obesitas wordt energie opgeslagen in het vetweefsel omwille van een onevenwicht tussen energie verbruik en energie opslag. Ten gevolge van aanhoudende gewichtstoename zal zich vetweefsel dysfunctie ontwikkelen, hetgeen wordt geassocieerd met ectopische vetstapeling en de ontwikkeling van metabole complicaties zoals insuline resistentie en diabetes mellitus type 2 (Hoofdstuk 1). Met oog op het behoud van een gezonde vetweefselfunctie bij personen met obesitas is een adequaat vetmetabolisme ter hoogte van het vetweefsel van noodzakelijk. Verbeteren van vetmobilisatie (en andere functionele vetweefsel kenmerken) is bijgevolg van primair belang in functie van preventie en behandeling van obesitas en de hiermee gepaarde metabole aandoeningen. Gezien het belang van een strikte regulatie van de vetmobilisatie (ook vetweefsel lipolyse genoemd), bestudeerden de studies beschreven in het huidige proefschrift de hormonale regulatie van de vetmobilisatie ter hoogte van het vetweefsel. Verder werd de invloed van een trainingsinterventie (en de combinatie met farmacologische inhibitie van vetweefsel lipolyse) op de vetweefsel lipolyse onderzocht bij personen met obesitas en werd het verband tussen vetweefsel lipolyse en obesitasgerelateerde insuline resistentie bestudeerd met oog op de potentiële relevantie binnen de behandeling en preventie van obesitas.

In de vakliteratuur is er steeds meer bewijs voor het feit dat natriuretische peptiden een rol van betekenis hebben binnen de metabole controle door hun effecten ter hoogte van insuline gevoelige weefsels zoals het vetweefsel, het spierweefsel en de lever, wat deze peptiden aantrekkelijk maakt binnen het humaan metabool onderzoek. Gezien hun rol in het reguleren van vetweefsel lipolyse (Hoofdstuk 2), leidde dit ons ertoe om te onderzoeken of natriuretische peptiden, en specifiek het atriaal natriuretisch peptide (ANP), betrokken zijn in de fysiologische regulatie van vetweefsel lipolyse bij obese, insuline resistente personen. 
In Hoofdstuk 3 werd een verstoorde maximale responsiviteit voor ANPgemedieerde vetcel lipolyse beschreven in geïsoleerde, mature subcutane, niet viscerale, vetcellen van obese mannen met of zonder type 2 diabetes, in vergelijking met slanke mannen met een vergelijkbare leeftijd. Deze verstoring kan (deels) verklaard worden door verschillende expressieprofielen van receptoren voor natriuretische peptiden (naast veranderingen post-receptor signaalmoleculen) bij obese insuline resistente individuen. Opmerkelijk genoeg was het depot-afhankelijk effect, aanwezig bij vetcellen van slanke mannen, waarbij subcutane vetcellen meer responsiviteit vertoonden voor ANP dan viscerale vetcellen, niet aanwezig bij obese mannen, hetgeen wellicht het behoud van overtollige vetmassa bij personen met obesitas in de hand werkt.

Naast een adequaat vetmetabolisme ter hoogte van het vetweefsel kunnen andere kenmerken van een dysfunctioneel vetweefsel (bv. vetcel hypertrofie, vetweefsel inflammatie) eveneens bijdragen aan een verhoogd cardio-metabool risico bij personen met obesitas. In Hoofdstuk 4 werd het belang van viscerale vetcel hypertrofie en viscerale B-lymfocyten als onafhankelijke variabelen voor insuline resistentie (bepaald aan de hand van de surrogaat index HOMA-IR) aangetoond. Ter hoogte van het subcutaan vetweefseldepot droeg voornamelijk de vetmassa, meer dan andere merkers van vetweefselfunctie, bij aan de variatie in insuline resistentie. De subklinisch inflammatoire status van het vetweefsel, hetgeen prevalent aanwezig is bij obese individuen, werd verder bevestigd door een verhoogde aanwezigheid van totale lymfocyten alsook proinflammatoire M1 macrofaag populaties in het visceraal vetweefseldepot van personen met obesitas (in vergelijking met slanke personen). Deze bevindingen geven aan dat er in het obese vetweefsel inderdaad een georkestreerd samenspel tussen verschillende celtypes aanwezig is.

Zoals beschreven in Hoofdstuk 3, werd aan de hand van mechanistische in vitro (ex vivo) methoden een ANP-resistent fenotype van het subcutaan vetweefsel bij personen met obesitas aangetoond. De fysiologische relevantie van de ANP-gemedieerde lipolyse tijdens inspanning werd vervolgens onderzocht gebruik makend van "gouden standaard" microdialyse experimenten ter hoogte van het abdominaal subcutaan vetweefsel van personen met obesitas. De bevindingen uit Hoofdstuk 5 wijzen op een belangrijke aandeel 
van niet-adrenerge regulatoren in het lipolytisch proces ter hoogte van de subcutane vetcel tijdens laag-intense uithoudingsinspanning bij personen met obesitas, vermoedelijk ANP-gemedieerd. Echter, de verstoringen op gebied van vetcel lipolyse bleven aanwezig na een gecombineerd trainingsprogramma, ondanks positieve veranderingen in metabool profiel en lichaamssamenstelling ten gevolge van dit trainingsprogramma bij personen met obesitas, hetgeen erop duidt op de nood aan geoptimaliseerde therapeutische strategieën ter bevordering van de preventie en behandeling van obesitas. Bijgevolg werd in Hoofdstuk 6 de toepasbaarheid en klinische effectiviteit van een gecombineerde aanpak aangetoond. Deze combinatietherapie omvatte het farmacologisch verlagen van de circulerende vrije vetzuren, gebruik makend van acipimox (een analoog van nicotinezuur) om de vetweefsel lipolyse te remmen, naast een laag- tot matig-intense uithoudingsinspanning in gevaste toestand bij obese personen met type 2 diabetes en resulteerde in een substantiële verbetering van de glycemie en insulinemie tijdens de daaropvolgende postprandiale fasen.

Uit de resultaten die in dit proefschrift werden behaald kan worden geconcludeerd dat een adequaat regulatie van het lipidenmetabolisme ter hoogte van het vetweefsel van cruciaal belang is om een 'gezonde' vetweefselfunctie na te streven. In dit proefschrift werd ook de fysiologische rol van ANP in de regulatie van vetcel lipolyse beschreven, voornamelijk in obesitasgerelateerde metabole aandoeningen zoals insuline resistentie of type 2 diabetes mellitus, condities waarbij een verstoorde catecholamine- en ANP-gemedieerde regulatie van vetcel lipolyse werd waargenomen op het niveau van het abdominaal subcutaan vetweefsel. De geobserveerde lipolytische defecten konden niet worden geremedieerd door middel van trainingsinterventie, hetgeen suggereert dat er een essentiële nood is aan nieuwe therapieën met als doel de klinische effectiviteit van (al dan niet gecombineerde) preventie- en behandelingsstrategieën te optimaliseren in functie van een verbeterde zorg voor personen met obesitas. 


\section{Curriculum Vitae}

Kenneth Verboven was born on $23^{\text {rd }}$ of January 1989 in Sint-Truiden, Belgium. After completing secondary school at Royal Atheneum 1 Hasselt, Belgium, he started, in 2007, a Bachelor in Biomedical Sciences at the Transnational University Limburg (tUL), a unique partnership of two universities in two countries: Hasselt University and Maastricht University. In 2012, he obtained his Master of Science in Biomedical Sciences at the tUL, Belgium/The Netherlands. In 2012, he worked as a scientific researcher at the Jessa Hospital in Hasselt, Belgium. In January 2013, Kenneth Verboven started as a joint PhD candidate at the Department of Rehabilitation Sciences of the Biomedical Research Institute of Hasselt University, Belgium, and the Department of Human Biology of Maastricht University Medical Centre + (MUMC+), the Netherlands, under the supervision of Prof. dr. Ellen Blaak and Prof. dr. Dominique Hansen. His research focuses on the endocrine regulation of adipose tissue lipolysis in human in vivo studies including fundamental in vitro methodology. His work has been presented at several national and international conferences (European Obesity Summit 2016, 52 ${ }^{\text {nd }}$ Annual Meeting of the European Association for the Study of Diabetes (EASD) and 24th European Congres on Obesity 2017). In 2017, he received a Travel Scholarship for the annual ECO meeting 2017, Porto, Portugal. 


\section{List of publications}

- Wouters K, Gaens K, Bijnen M, Verboven K, Jocken J, Wetzels S, Wijnands E, Hansen D, van Greevenbroek M, Duijvestijn A, Biessen E, Blaak E, Stehouwer C, Schalkwijk C. Circulating classical monocytes are associated with $C D 11 c+$ macrophages in human visceral adipose tissue. Sci. Rep. 7, 42665; doi: $10.1038 /$ srep42665

- Marinus N, Bervoets L, Massa G, Verboven K, Stevens A, Takken T, Hansen D. Altered gas-exchange at peak exercise in obese adolescents: implications for verification of effort during cardiopulmonary exercise testing. J Sports Med Phys Fitness 2016; Epub ahead of print

- Wens I, Dalgas U, Vandenabeele F, Verboven K, Hansen D, Deckx N, Cools N, Eijnde BO. High Intensity aerobic and resistance exercise can improve glucose tolerance in persons with multiple sclerosis: a randomized controlled trial. Am J Phys Med R

- Verboven K, Hansen D, Moro C, Eijnde BO, Hoebers N, Knol J, Bouckaert W, Dams A, Blaak EE, Jocken JWE. Attenuated atrial natriuretic peptide-mediated lipolysis in subcutaneous adipocytes of obese type 2 diabetic men. Clinical Science 2016; 130(13):1105-14ehabil 2017; 96(3):161-166

- Wens I, Dalgas U, Verboven K, Kosten L, Stevens A, Hens N, Eijnde BO. Impact of high intensity exercise on muscle morphology in EAE rats. Physiol Res 2015; 64(6):907-23

- Wens I, Dalgas U, Vandenabeele F, Grevendonk L, Verboven K, Hansen D, Van Wijmeersch B, Eijnde BO. High-intensity exercise in multiple sclerosis: effects on muscle contractile characteristics and exercise capacity, a randomized controlled trial. PLoS One 2015:e0133697

- Stevens A, Ferferieva V, Bito V, Wens I, Verboven K, Deluyker D, Voet A, Vanhoof J, Dendale P, Eijnde BO. Exercise training improves cardiac function and attenuates insulin resistance in Dahl saltsensitive rats. Int J Cardiol 2015; 186:154-60

- Hansen D, Linsen L, Verboven K, Hendrikx M, Rummens JL, van Erum M, Eijnde BO, Dendale P. Magnitude of muscle wasting early after on-pump coronary artery bypass graft surgery and exploration of aetiology. Exp Physiol 2015; 100: 818-28

- Hansen D, Wens I, Vandenabeele F, Verboven K, Eijnde BO. Altered signaling for mitochondrial and myofibrillar biogenesis in skeletal muscles of multiple sclerosis patients. Transl Res 2015; 166: 70-9

- Wens I, Hansen D, Verboven K, Deckx N, Kosten L, Stevens A, Cools N, Van Wijmeersch B, Eijnde BO. Impact of 24-weeks of resistance and endurance exercise on glucose tolerance in persons with multiple sclerosis. Am J Phys Med Rehabil 2015; 94: 838-47

- Hansen D, Wens I, Keytsman C, Verboven K, Dendale P, Eijnde BO. Ventilatory function during exercise in multiple sclerosis and impact of training intervention: cross-sectional and randomized controlled trial . Eur J Phys Rehabil Med 2015; 51(5):557-568 
- Hansen D, Wens I, Kosten L, Verboven K, Eijnde BO. Slowed exercise-onset VO2 kinetics during submaximal endurance exercise in subjects with multiple sclerosis. Neurorehabil Neural Repair 2013; 27: 87-95

\section{In preparation/Submitted}

- Verboven K, Wouters K, Gaens K, Hansen D, Bijnen M, Wetzels S, Stehouwer C, Goossens G, Schalkwijk C, Blaak E, Jocken J. Abdominal subcutaneous and visceral adipocyte morphology, lipolysis and inflammation in relation to insulin resistance in obese humans. Submitted

- Verboven K, Jocken JWE, Hansen D, Blaak EE. Natriuretic peptides in control of body weight and insulin sensitivity. In revision

- Verboven K*, Hansen D*, Van Dijk JW, Zorenc A, Minten L, Smeets K, Van Loon L. Adipose tissue lipolysis inhibition during endurance exercise leads to greater acute improvements in glycemic control in male type 2 diabetes patients. Submitted * shard first authorship

- Verboven K*, Stinkens R*, Hansen D, Wens I, Frederix I, Eijnde BO, Jocken J, Goossens G, Blaak E. Coordinated regulation of adipose tissue adrenergic- and non-adrenergic-mediated lipolysis during exercise in lean and obese individuals: the effect of exercise training. In preparation

- Warnke I, Schwager J, Verboven K, Hooiveld G, Schoop R, Hoebers N, Essers Y, Toepfer C, Blaak E, Jocken J. Effects of combining lycopene and resveratrol on functional features of in-vitro differentiated human white adipocytes. In preparation

- Keytsman C, Eijnde BO, Verboven K, Hansen D, Wens I. Increased fat mass is the predominant cardiovascular risk factor in Multiple Sclerosis. Submitted

- Hansen D, Boujemaa H, Verboven K, Hendrikx M, Rummens J, Frederix I, Eijnde BO, Dendale P. Muscle wasting after coronary artery bypass graft surgery: impact on post-operative clinical status and effect of exercise-based rehabilitation. Submitted 
Dankwoord 
Ondanks het feit dat ik een persoon ben van weinig woorden wil ik via dit dankwoord toch enkele woorden richten aan de prachtige mensen die ik tijdens mijn doctoraatsonderzoek (beter) heb leren kennen. Ik had het geluk aan twee onderzoeksinstellingen te mogen werken, en dus dubbel zoveel leuke collega's met stuk voor stuk een aandeel in dit geheel, waar ik iedereen héél dankbaar voor ben!

Ellen, dankjewel dat je me in 2013 wilde ontvangen om samen dit gezamenlijk PhD-project uit de grond te stampen. Vanaf de start kreeg ik een gevoel van vertrouwen, ook al was het weer een Belg die in je lab terecht was gekomen. Heel dankbaar ben ik voor de kans die ik in jouw groep kreeg, dit opende heel wat perspectieven waar we in Hasselt nooit aan hadden durven denken bij de start van het project gezien onze onervarenheid in het humaan metabool onderzoek. Ik heb nog steeds veel bewondering voor al je wetenschappelijke inzichten die onze studieprotocollen na elk overleg weer net dat tikkeltje verbeterden, ook al moest er soms worden terug gegaan naar het oorspronkelijk idee :) Bedankt Ellen voor de leerrijke, maar ook gezellige jaren, welke ik nog lang zal blijven koesteren! Ik kijk er alvast heel erg uit om onze samenwerking te blijven onderhouden.

Dominique, bedankt voor alles! Al in 2012 maakte ik, tijdens mijn seniorstage op REVAL, kennis met je onuitputtelijke gedrevenheid en ambities. Dit werkte zo aanstekelijk dat ik graag inging op je vraag om bij jou een PhD te beginnen, een beslissing waar ik geen moment over heb getwijfeld! Bedankt dat je me van het begin de vrijheid en het vertrouwen hebt gegeven om zelfstandig mijn ding te doen. Altijd stond je deur open, al kwam ik weer een keer vragen om een (dure) bestelling te plaatsen. Je enthousiasme telkens ik met nieuwe analyses aan kwam lopen werkte inspirerend, al moest ik je wilde ideeën voor nieuw onderzoek soms wat temperen :) Tot op vandaag bewonder ik nog steeds je passie waarmee je telkens nieuwe studies uitdenkt, uitvoert en opschrijft. Dominique, een dikke merci!

Johan, ik wil je graag heel erg bedanken voor al je inzichten en ondersteuning om mij vertrouwd te maken met het hele lipolyse-gebeuren! Je kritische blik op 
telkens nieuwe versies van papers zijn van goudwaarde, alsook de motiverende woorden op het einde van al onze overlegmomenten. De aanpassing naar de Nederlandse manier van werken verliep 'smooth' mede dankzij jouw waardevolle tips die je me regelmatig hebt gegeven. Een welverdiende dank $u$ wel voor je bijdrage in dit geheel!

Bert, ook voor jou alleen maar woorden van dank. Je kennis omtrent de inspanningsfysiologie heeft mijn biomedische kennis heel erg verreikt. Je introduceerde me destijds in het klinische werkveld, een gouden combinatie met het fundamentele onderzoek bleek achteraf! Je stond altijd klaar om een logistieke oplossing te bedenken wanneer ik al je apparatuur weer een keer nodig had om proefpersonen te testen, nooit was dit teveel gevraagd (wat ik heel erg heb weten appreciëren). Ik hoop dat we in de toekomst onze expertises verder kunnen delen! Bert, bedankt!

I would also like to thank the members of the Assessment Committee and the corona of my thesis defence, Prof. Marleen van Baak, Prof. Mikael Rydén, Prof. Patrick Rensen, Prof. Marleen van Greevenbroek, Prof. Paul Dendale, Prof. Virginie Bito for your time and effort that you put in reading and reviewing this thesis.

Ik bedank ook graag mijn co-auteurs voor hun input en kritische commentaren om de papers in deze thesis te vormen tot hoe ze nu zijn geworden. Cédric, un grand remerciement pour toutes les recommendations sur les expérimentations in vitro. Kristiaan, Katrien, Coen en Casper, bedankt om me wegwijs te maken in de wondere wereld van de inflammatie. Gijs, heel erg bedankt voor je nuttige suggesties om in de manuscripten de nuances net wat anders aan te brengen. Je kwam telkens weer met nieuwe interpretaties van onze resultaten, al was het op de gang of met een biertje op een of ander congres. Het was een plezier om met je samen te werken, bedankt Gijs! Luc, Lex en Jan-Willem, bedankt om telkens tijd te maken om ons gezamenlijk verhaal steeds weer sterker te maken. Dit heeft mijn visie op wat dingen zeker verbreed, wat in de toekomst nog zijn nut zal bewijzen.

Bedankt ook aan de mensen van de afdeling Cardiologie, het Hartcentrum Hasselt en het Revalidatie- en Gezondheidscentrum van het Jessa Ziekenhuis. In 
het bijzonder wil ik dr. Dendale bedanken om telkens de mogelijkheid te bieden al onze metingen en experimenten in een veilige, perfect uitgeruste omgeving te laten plaats vinden. Een expert in eigen gebied met een passie voor zijn vak en wetenschappelijk onderzoek, buitengewoon bewonderenswaardig! Natuurlijk wil ik ook Jan, Kim, Katrien, Ines, An, Frank en Toon van harte bedanken voor de talrijke, gezellige babbels in het ReGo. Ook al was ik een 'buitenstaander', ik heb me steeds welkom gevoeld en was zeer blij om deel te mogen uitmaken van jullie ROPARUN-team (\#blijdatjeinmijnteamzit)!

In de eerste fase van mijn promotietraject heb ik het voorrecht gehad om samen te werken met de afdelingen Abdominale Heelkunde van het Jessa Ziekenhuis en het Ziekenhuis Oost-Limburg. Daarom een welgemeende dankjewel aan dr. Knol, dr. Bouckaert, dr. Dams, dr. Vangertruyden, dr. Houben en dr. Verhelst. Sandra, Peggy en Marleen, bedankt voor het steeds tijdig aanleveren van jullie patiëntgegevens!

Anne, Frank en Ines, jullie hebben ook een groot aandeel in dit verhaal. Onze samenwerking zat steeds goed, ik kon telkens op jullie hulp en expertise rekenen wanneer er weer eens een bloedafname of een biopt moest gebeuren. Bedankt!

Mijn paranimfen. Inez, je was degene bij wie mijn onderzoeksloopbaan is begonnen. Als student BMW wist je me in 2012 te overtuigen om met dit doctoraatsproject te starten, bedankt daarvoor! We hebben een lange, maar gezellige, tijd samen de bureau gedeeld (samen met An), waar je ook altijd klaar stond om een handje te helpen wanneer het nodig was. Het was een leuke tijd en ik ben blij dat je, mede in functie als mijn paranimf (ook al is dit voor ons Belgen een nieuwigheid), samen dit hoofdstuk met mij mag afsluiten! Ik hoop dat we nog veel contact zullen houden! Rudi, we hebben mekaar vooral beter leren kennen in de tweede fase van onze PhD's. Gelukkig, want je was (en bent nog steeds) een topcollega! Tijdens onze gezamenlijke studie was het een plezier om steeds aan een testdag te starten, ook al was het weer vroeg in de ochtend. Ook onze belevenissen tijdens de ECO zullen altijd bij blijven (Gothenburg Memories) ;) Bedankt voor je bijdrage in dit geheel! Inez en Rudi, ik wens jullie alle geluk en veel succes toe in jullie verdere leven! 
Mijn CRI collega's. De afgelopen 4 (en zelf 5, als ik terug denk aan mijn laatstejaarsstage als student BMW) jaren waren fantastisch! Niet alleen op professioneel vlak vormen we een hecht team waarin iedereen steeds voor mekaar klaar staat en waarbij geen inspanning teveel is, maar ook daarbuiten zijn jullie allemaal topmensen! De gezellige CRI-etentjes, de vrijdagavond borrels en verschillende andere randactiviteiten maakten deel uit van het geheel, een betere werkplek kan ik me niet wensen. Bedankt Inez, An, Jana, Lisa, Charly, Chris, Pieter, Dominique, Martijn en Bert!

Mijn collega's van HB. Mijn tijd bij HB was een geweldige ervaring. Als nieuweling werd ik meteen in jullie groep opgenomen. Gezien mijn beperkte expertise werd ik tijdens de afgelopen jaren omringd door experten in hun domein, waarbij jullie me met raad en daad bijstonden wanneer ik weer een (voor mij) nieuwe analyse moest uitvoeren. Vooral de inzet van onze gezellige en über sympathieke laboranten Nicole en Yvonne zijn van onschatbare waarde. Congressen werden ervaren als citytrips, waar we memorabele momenten beleefden (en hopelijk nog zullen beleven)! Bedankt Rudi, Johan, Gijs, Nicole, Yvonne, Max, Birgitta, Dorien, Emmanuel, Ruth, Jasper, Mattea, Ellen, Manuel, Adriyan en Rens voor de leuke tijd!

Graag wil ik ook de leden van de vakgroep RWK, met in het bijzonder Peter en Monique, bedanken voor hun vertrouwen en steun. Ook alle andere REVALcollega's wil ik hierbij bedanken voor de geweldige tijd die we samen hebben beleefd. De sfeer onder de 'jonge garde' op de eerste verdieping van gebouw A was altijd top. Tussen de wetenschappelijke discussies door was er steeds ruimte voor een grap, een drink of een collectieve sportprestatie. Bedankt Jonas, Anouk, Rob, Wout, Lousin, Steffi, Fanny, Deborah, Liesbet, Tom, Ilse L, Bart, Ilse B, Inez, Joke, Koen, Lotte en natuurlijk mijn eigen bureaumaatjes van A1.06!

De mensen van Universitaire Biobank Limburg verdienen natuurlijk ook een woord van dank. Dr Rummens, Loes, Tine, bedankt om steeds klaar te staan en me verder te helpen bij de opslag van onze stalen of het uitvoeren van onze analyses. Ook een dank $u$ wel aan alle laboranten van het klinische labo van 
het Jessa Ziekenhuis om altijd met een glimlach onze stalen te verwerken en ons tijdig de nodige uitslagen te bezorgen.

Geen onderzoek zonder toplaboranten! Mijn dank gaat daarom ook uit naar Jos, Wendy, Nicole, Yvonne, Antoine en Joan om steeds hun expertise te delen met mij en de gevraagde analyses in een recordtijd uit te voeren! Ook bedankt aan Paul, je zorgde steeds voor de nodige voorraad stikstof zodat ik vroeg in de ochtend steeds kon starten met mijn experimenten! Ik heb veel van jullie geleerd, dit zal ik de rest van mijn loopbaan meedragen!

Deze thesis was niet tot stand gekomen zonder de vrijwillige deelname van mijn proefpersonen. Ik ben me er van bewust dat er veel van jullie werd gevraagd: bloed, zweet, vet, spier, scans enz. Wat voor mij vanzelfsprekend was, was voor jullie iets nieuws. Graag wil ik alle deelnemers bedanken voor hun inzet en vrijwillige deelname aan al onze studies!

Een warm en verwelkomend secretariaat is eveneens deel van de geoliede machine en is dus van groot belang geweest tijdens de totstandkoming van deze thesis. Anne, Maya, Veronique, Rani, Desiree, Cleo en Yolanda, jullie weten op ieders probleem een oplossing te vinden. Jullie zijn de matresfamilias van de afdelingen waar je altijd terecht kan, ook al is het maar voor een korte (maar gezellige) babbel! Bedankt!

Doorheen de afgelopen promotiejaren was er op tijd en stond nood aan afleiding, om alle zorgen van het onderzoek en de deadlines even te vergeten en de gedachten te verzetten. Hier hebben heel wat vrienden hun steentje toe bijgedragen, ook al moesten ze telkens opnieuw hetzelfde verhaal aanhoren. Bedankt aan alle BMW-vrienden, 'De mannen van Biomedische' en alle badminton- en niet-badmintonvrienden!

Ten slotte wil ik mijn familie graag dubbel en dik bedanken voor de steun en raad doorheen de afgelopen jaren, maar ook al de jaren die hieraan vooraf zijn gegaan. Ik zou hier nooit gestaan hebben zonder jullie! Jullie, en ook mijn schoonfamilie, waren ook steeds bereid om de jaarlijkse bbq's en verjaardagsfeestjes op een moment in te plannen zodat ik er toch steeds kon bij 
zijn, dit heb ik altijd weten te appreciëren! Mama, Papa, Bomma, Oma, Bompa, Opa, Pa en Kimberley, ook al hadden jullie vaak geen idee waarmee ik al die tijd precies mee bezig was, jullie waren altijd oprecht geïnteresseerd, trots en wisten me steeds te motiveren. Jullie deden me altijd weer opnieuw beseffen dat er sommige dingen en vooral sommige personen belangrijker zijn dan werk. BEDANKT!

Liefste Anne, voor u alleen maar de allergrootste woorden van dank. Bedankt voor je begrip als ik 's avonds of in het weekend moest/wilde werken of wanneer ik weer voor enkele dagen naar het buitenland moest. Nooit is iets je teveel, je staat altijd voor mij (en alle anderen) klaar. Het gezegde "achter elke man staat een sterke vrouw" is je op het lijf geschreven, getuige hiervan je onvermoeibare inzet om ons toekomstig huisje in orde te krijgen (ook al had ikzelf hier het afgelopen jaar jammer genoeg weinig tijd voor)! Bedankt voor je liefde en om er altijd te zijn! Binnenkort is het onze beurt om te gaan genieten in ons stulpje op de Bosstraat! 